\title{
SIGNS，LANGUAGE AND BEHAVIOR
}

by

\section{CHARLES MORRIS \\ University of Chicago}

New York PRENTICE-HALL, INC. 1946 
Copyright, 1946, by

PRENTICE-HALL, INC.

70 Fifth Alenue, New York

All rights reserved. No part of this book may be reproduced in any form, by mimeograph or any other means, without permission in writing from the publisher.

Second Printing August, 1947

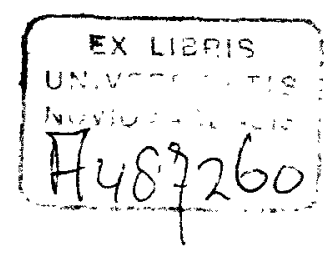

Printed in the United States of America 


\section{PREFACE AND ACKNOWLEDGMENT}

7 HIS book aims to lay the foundation for a comprehensive and 1 fruitful science of signs. It attempts to develop a language in which to talk about signs, whether the signs be those of animals or men; whether or not they themselves constitute a language; whether they are signs in science or signs in art, technology, religion, or philosophy; whether they are healthy or pathic, adequate or inadequate for the purposes for which they are used.

The book is written from the point of view first expressed by Charles Peirce, that to determine the meaning of any sign "we have ... simply to determine what habits it produces." Signs are therefore described and differentiated in terms of the dispositions to behavior which they cause in their interpreters. The approach is, in a wide sense of the term, behavioral, and owes much to the theories of behavior developed by George H. Mead, John Dewey, Edward C. Tolman, and Clark L. Hull. But the logicians, too, have in their own way contributed greatly to the understanding of signs, and our account also draws upon their results, especially upon the analyses of Rudolf Carnap.

A comprehensive theory of signs must take into account the work of linguists, estheticians, psychopathologists, and social scientists. Since no person can be trained adequately in all these fields, the science of signs must be developed co-operatively. I have been fortunate in obtaining such co-operation to a remarkable degree during the various rewritings of this work. And for this co-operation I am very grateful.

Rudolf Carnap, C. J. Ducasse, Lewis E. Hahn, Clark L. Hull, Abraham Kaplan, Willis Moore, I. A. Richards, and Edward C. Tolman were careful critics of various parts, or the whole, of earlier versions of the book. And in conversation or comments on special chapters I have received throughout the years many valuable suggestions from Manuel J. Andrade, Max Black, Leonard Bloomfield, Frank G. Bruner, Egon Brunswik, Karl Bühler, Norman Dalkey, Estelle DeLacy, John Dollard, Alfred E. Emerson, Erich Fromm, Ruth Herschberger, Hein- 
rich Klüver, Ernst Kris, Harold D. Lasswell, C. I. Lewis, George K. Link, Donald G. Marquis, Jules H. Masserman, Mark A. May, Howard Parsons, Hans Reichenbach, Benbow F. Ritchie, Valerie Saiving, H. M. Sheffer, Milton B. Singer, Harry Stack Sullivan, and Donald C. Williams. None of these persons saw the final text, and so although they have contributed much to whatever is good in this book, they cannot be blamed for what is bad.

The typing and editing of the various rewritings, and research in connection with certain topics, was done by Ruth Herschberger. Bess Sondel put the Bibliography into final form and compiled the Index. Howard Parsons and Helen Brummall aided in the preparation of the final typescript.

The John Simon Guggenheim Memorial Foundation and The Rockefeller Foundation provided me with the freedom necessary for writing this book; the American Council of Learned Societies contributed to the expense of preparing the final version of the manuscript.

I am grateful to the publishers for permission to quote from the following books: Harvard University Press: Collected Papers of Charles Sanders Peirce, edited by Charles Hartshorne and Paul Weiss; Susanne K. Langer, Philosophy in a New Key; Rudolf Carnap, An Introduction to Semantics; the University of Chicago Press: George H. Mead, Mind, Self, and Society, edited by Charles Morris; Jules H. Masserman, Behavior and Neurosis; Oxford University Press: I. A. Richards, The Philosophy of Rhetoric; Columbia University Press: Ray Lepley, Verifiability of Value; D. Appleton-Century Company, Inc.: Edward C. Tolman, Purposive Behavior in Animals and Men; E. R. Hilgard and D. G. Marquis, Conditioning and Learning; Clark L. Hull, Principles of Behavior; Harcourt, Brace and Company, Inc., and Kegan Paul, Trench, Trubner \& Company, Ltd.: C. K. Ogden and I. A. Richards, The Meaning of Meaning; Doubleday and Company, Inc.: Walt Whitman, "Song of Myself," Leaves of Grass; Yale University Press: Robert M. Yerkes, Chimpanzees, A Laboratory Colony.

While the present study is comprehensive in scope, it is far from exhaustive. But it does show, I believe, how signs may be studied behaviorally and the relevance of such an approach for linguistics, esthesics, logic, psychopathology, education, and philosophy, as well as for 
the general field of the socio-humanistic disciplines and for the unification of science as a whole.

The development of the science of signs is the cause which this work intends to serve. It is hoped that it will contribute at this time the kind of stimulation that has been provided for several decades by C. K. Ogden's and I. A. Richards' The Meaning of Meaning. The building of a comprehensive and fruitful science of signs will continue to be the task of many investigators working in many fields for many generations.

Charles Morris 


\section{CONTENTS}

Preface and Acknowledgment . . . . . . . . $\mathrm{v}$

I. Signs AND Behavior . . . . . . . . . 1

I. The Problem of Approacb . . . . . . . . 1

2. Preliminary Isolation of Sign-Bebavior. . . . 5

3. Toward Precision in the Identification of SignBebavior . . . . . . . . . . 8

4. Some Objections Considered . . . . . . . 11

5. Furtber Remarks on Sign-Processes . . . . . 15

6. The Basic Terms of Semiotic . . . . . . . 17

7. Extension of Terminology . . . . . . . 20

8. Signal and Symbol . . . . . . . . . . 23

9. Alternatives to a Behavioral Semiotic . . . . 27

II. Language and Social Behavior . . . . . 32

1. Language as Sign Pbenomenon . . . . . . . 32

2. The Definition of 'Language' . . . . . . . 35

3. Consideration of the Proposed Definition . . . 36

4. The Interpersonality of the Language Sign . . . 39

5. Mead's Concept of the Significant Symbol . . . 42

6. Post-Language Symbols . . . . . . . . . 46

7. The Mentalistic Controversy Resumed . . . . 49

8. Signs in Animals and Men . . . . . . . 52

9. Language and Behavioristics . . . . . . 55

III. Modes of Signifying . . . . . . . . 60

I. The Problem Stated . . . . . . . . . 60

2. Origin of the Modes of Signifying . . . . . $\quad 62$

3. Behavioral Criteria for Differentiating the Modes . 64

4. Expression, Emotion, and Usage . . . . . 67

5. Ogden and Richards on Modes of Signifying . . 69

6. Ascriptors . . . . . . . . . . . 72

7. Identifiors, Designators, and Designative Ascriptors 76

8. Appraisors and Appraisive Ascriptors . . . . 79

g. Prescriptors and Prescriptive Ascriptors . . . . 83 
10. Formators and Formative Ascriptors . . . . 86

II. Interrelation of the Modes of Signifying. . . . 88

IV. Adequacy, Truth, and Reliability of Signs . 92

I. The Sense in Which a Sign Is Used . . . . 92

2. The Four Primary Sign Usages . . . . . . . 95

3. Informative Adequacy: Convincingness . . . . 97

4. Valuative Adequacy: Effectiveness . . . . . 99

5. Incitive Adequacy: Persuasiveness . . . . . 102

6. Systemic Adequacy: Correctness . . . . . . . 104

7. T-Ascriptors and "Trutb" . . . . . . . 106

8. Belief and Knowledge . . . . . . . . 108

9. The Limits of Signification and Knowledge . . 111

10. The Control of Non-Designative Ascriptors . . . 114

II. Communication . . . . . . . . . . 118

12. Belief, Denotation, and Adequacy . . . . . 121

V. Types of Discourse . . . . . . . . . 123

I. Classificatory Basis . . . . . . . . . . . . 123

2. Scientific Discourse . . . . . . . . . . 126

3. Fictive Discourse . . . . . . . . . . . 128

4. Legal Discourse . . . . . . . . . . 130

5. Cosmological Discourse . . . . . . . . 132

6. Mythological Discourse . . . . . . . . . 134

7. Poetic Discourse . . . . . . . . . . 136

8. Moral Discourse. . . . . . . . . . . . 138

9. Critical Discourse . . . . . . . . . . 140

10. Technological Discourse . . . . . . . . . 143

1I. Political Discourse . . . . . . . . . . 145

12. Religious Discourse . . . . . . . . . 146

13. Propagandistic Discourse . . . . . . . 148

14. The Study of Types of Discourse . . . . . 150

VI. Formators and Formative Discourse . . . 153

I. The Problem of Formators . . . . . . . . . . 153

2. The Nature of Formators . . . . . . . . 156

3. Kinds of Formators . . . . . . . . . . 159

4. Formative Ascriptors . . . . . . . . . 162

5. The Behavioral Basis of Formative Ascriptors . . 164

6. Formative Ascriptors, Truth, and Knowledge . . 166 
7. Logico-Matbematical Discourse . . . . . . 168

8. Rbetorical Discourse . . . . . . . . . 171

9. Grammatical Discourse . . . . . . . . 173

10. Metapbysical Discourse . . . . . . . . 175

II. Logic and Matbematics . . . . . . . . 178

12. Rbetoric, Grammatic, and Metaphysics . . . 182

VII. Individual and Social Import of Signs . . 187

I. The Problem of This Chapter . . . . . . 187

2. The Significance of Non-Vocal Signs . . . . . 190

3. Are the Arts Languages? . . . . . . . 192

4. Effects of Personal Post-Language Symbols . . 196

5. The Pathology of Signs . . . . . . . . . . 198

6. Signs and Personality Disturbances . . . . 201

7. Signs and Societies . . . . . . . . . 204

8. Social Control . . . . . . . . . . 207

9. The Social Patbology of Signs . . . . . . 210

I0. Communication, Co-operation, and Conflict . . . 212

VIII. The Scope and Import of Semiotic . . . . 217

1. Pragmatics, Semantics, and Syntactics . . . . 217

2. A Program for Linguistics . . . . . . . . 220

3. Semiotic as Unification of Science . . . . . . 223

4. The Place of Psychology within Science. . . . 227

5. The Humanities and Scientific Humanistics . . 230

6. The Language of Philosopby . . . . . . . 233

7. The Import of Semiotic for the Individual . . . 239

8. The Import of Semiotic for Social Organization . 242

9. Semiotic and the School. . . . . . . . 244

Io. Problems and Program . . . . . . . . 246

Notes to Chapters . . . . . . . . . . . . . . 249

Appendix: Some Contemporary Analyses of Sign-

Processes . . . . . . . . . . . . . . . 285

1. The History of Semiotic . . . . . . . . 285

2. Charles Peirce on Signs . . . . . . . . . 287

3. Behavioral Formulations of Sign-Processes . . . 291

4. Mentalistic Formulations of Sign-Processes . . 295 
5. Consideration of the Behavioral-Mentalistic Controversy. . . . . . . . . . . . 299

6. Tolman's Concept of Sign-Gestalt . . . . . . 302

-. Hull's Concept of Pure-Stimulus Acts . . . . . 305

8. The Uniqueness of Sign-Bebavior . . . . . 308

Bibliography . . . . . . . . . . 311

GLOSSARY . . . . . . . . . . . 345

INDEX 
SIGNS, LANGUAGE, AND BEHAVIOR 


\section{SIGNS AND BEHAVIOR SITUATIONS}

\section{The Problem of Approach}

THE understanding and the effective use of language and other 1 signs has become today an insistent task. Popular and technical literature is filled with discussions of the nature of language, the differences between signs in men and in animals, the differentiation of scientific discourse and such other types of disçourse as occur in literature, religion, and philosophy, the consequences of an adequate or inadequate use of signs for personal and social adjustments.

These discussions are carried on from many points of view and for various purposes. There are linguists, psychologists, and social scientists interested in the study of those special kinds of signs which occur in their own specific subject matters; philosophers eager to defend the superiority of one system of philosophy over another; logicians and mathematicians working on the elaboration of a symbolism adequate to their own special disciplines; artists and religionists anxious to justify in an age of science their own special symbols; educators desirous of improving the use of language in the educational process for which they are responsible; psychiatrists aiming to uncover the place which signs play in the personality disturbances of their patients; statesmen seeking to uphold or improve the basic symbols which sustain the social structure; propagandists attempting to discern the ways in which language may be used to direct social change.

All of these approaches and purposes are legitimate. Their multiplicity bears witness to the enormous place which signs play in human 
life, while the ferment of contemporary discussion about signs is one evidence of the tensions which beset our culture. Language is of such central importance that it becomes an object of central concern in times of extensive social readjustment. It is not surprising that in these days, as in the later Greek and Medieval periods, attempts should be made to develop a comprehensive doctrine of signs. Today this discipline is frequently known as semantics; we shall call it semiotic. ${ }^{\mathbf{A}}$

And yet it must be admitted that from a scientific, and therefore from a practical, point of view the present status of this discipline is far from satisfactory. We do not often have reliable knowledge to direct fruitfully the applications we would like to make and endeavor to make. The situation is similar to that which the physician frequently encounters: he must do what he can for a particular illness even if adequate scientific knowledge is lacking. It is obvious that we do not possess a developed science of signs, though there are beginnings of such a science in such special fields as linguistics. There are few general principles now available in terms of which existing knowledge can be integrated and from which empirically verifiable predictions can be made. Yet the pressing problems for which such a science would be the proper instrument cannot be disregarded until a developed science of signs is obtained.

In this situation it has seemed wise to adopt a compromise attitude. The need of furthering a genuine science of signs is so basic that we must move in this direction as speedily as possible. The present study is based on the conviction that a science of signs can be most profitably developed on a biological basis and specifically within the framework of the science of behavior (a field which, following a suggestion of Otto Neurath, may be called behavioristics). Hence I shall constantly suggest connections between signs and the behavior of animals and men in which they occur. On the other hand, behavioristics is not at present sufficiently developed to account adequately for the more complex human actions, nor for the signs which they utilize. Since our presentday problems demand, however, insight into just such complexities, I

\footnotetext{
${ }^{1}$ Capital letters in the text refer to the notes at the end of the volume. These notes deal with technical issues and may be ignored by the general reader.
} 
shall not hesitate to introduce considerations drawn from very gross observations. And everywhere I shall seek for a wide perspective which will help to co-ordinate the great variety of interests, scientific and cultural, in sign phenomena. I share the view that insight into the nature of signs provides us with an instrument which improves our understanding of, and effective participation in, the whole of our contemporary intellectual, cultural, personal, and social problems. In support of this conviction I advise the reader to turn to Chapter 7, which deals with the importance of signs in the life of individuals and societies; a preliminary reading of that material at this time will serve to "fortify" the reader for the analysis which lies ahead.

The following study is directed both to scientists and to those concerned with the basic issues of contemporary individual and social life. There is a need today for such a study, inadequate and incomplete though it is bound to be. It may serve as a guide to the genuinely scientific and culturally fertile semiotic which the future will bring.

There is wide disagreement as to when something is a sign. Some persons would unhesitatingly say that blushing is a sign, others would not. There are mechanical dogs which will come out of their kennels if one claps one's hands loudly in their presence. Is such clapping a sign? Are clothes signs of the personality of those who wear them? Is music a sign of anything? Is a word such as 'Go!' a sign in the same sense as is a green light on a street intersection? Are punctuation marks signs? Are dreams signs? Is the Parthenon a sign of Greek culture? Disagreements are widespread; they show that the term 'sign' is both vague and ambiguous. ${ }^{B}$

This disagreement extends to many other terms which are commonly used in describing sign-processes. The terms 'express,' 'understand,' 'refer,' 'meaning' would provide many instances. So would 'communication' and 'language.' Do animals communicate? If so, do they have a language? Or do only men have language? Yes, run some answers; no, run others. We find the same diversity of replies if we ask whether thought or mind or consciousness is involved in a signprocess; whether a poem "refers" to what it "expresses"; whether men 
can signify what cannot be experienced; whether mathematical terms signify anything; whether language signs are preceded genetically by non-language signs; whether the elements in an undeciphered "dead" language are signs.

In the face of such disagreements, it is not easy to find a starting point. If we are to seek for a formulation of the word 'sign' in biological terms, the task is to isolate some distinctive kind of behavior which agrees fairly well with frequent usages of the term 'sign.' Since usage of the term is, however, not consistent, it cannot be demanded that the chosen behavioral formulation agree with all the various usages which are actually current. At some point the semiotician must say: "Henceforth we will recognize that anything which fulfills certain conditions is a sign. These conditions are selected in the light of current usages of the term 'sign,' but they may not fit in with all such usages. They do not therefore claim to be a statement of the way the term 'sign' is always used, but a statement of the conditions under which we will henceforth admit within semiotic that something is a sign."

Then from such a starting point a behavioral theory of signs will build up step by step a set of terms to talk about signs (taking account of current distinctions but attempting to reduce for scientific purposes their vagueness and ambiguity), and will endeavor to explain and predict sign phenomena on the basis of the general principles of behavior which underlie all behavior, and hence sign-behavior. The aim is to take account of the distinctions and analyses which former investigators have made, but to ground these results whenever possible upon general behavior theory. In the nature of the case such a scientific semiotic will often deviate from current terminology, and can only be developed slowly and laboriously. It will often seem pedantic and less illuminating for many purposes than less scientific approaches-which therefore are to be encouraged in the light of the many problems and purposes which a treatment of signs aims to fulfill. It is not to be expected that all discussions of literary, religious, and logical signs can be translated at once with profit into a behavioral formulation. The present approach does not therefore wish to exclude other approaches to semiotic. But it does proceed upon the belief that basic progress in this complex field rests finally upon the development of a genuine science 
of signs, and that this development can be most profitably carried on by a biological orientation which places signs within the context of behavior.

\section{Preliminary Isolation of Sign-Behavior}

We shall begin by taking two examples of behavior to which the term 'sign' is often applied both in common usage and in the writings of semioticians. Then a superficial analysis of these examples will disclose the features which a more technical formulation of the nature of a sign must embody. If both situations reveal certain common elements, then both may be called sign-behavior; the differences in the two situations would then suggest differences between kinds of signs. If analysis shows too great differences, then the alternative would be to choose different terms to describe the two situations, and to adopt a narrower definition of 'sign': in either case we would then be in a position to consider whether any additional phenomena are to be called signs, that is, whether the characterization of signs based upon the two examples in question is to be held as a basis for determining when something is a sign or whether it is to be expanded to include situations of a widely different sort.

The first example is drawn from experiments on dogs. ${ }^{\mathrm{c}}$ If a hungry dog that goes to a certain place to obtain food when the food is seen or smelled, is trained in a certain way, it will learn to go to this place for food when a buzzer is sounded even though the food is not observed. In this case the dog is attentive to the buzzer but does not normally go to the buzzer itself; and if the food is not made available until some time after the buzzer has sounded, the dog may not go to the place in question until the time interval has elapsed. Many persons would say in such a situation that the buzzer sound is to the dog a sign of food at the given place, and in particular, a non-language sign. If we abstract from the experimenter and his purposes in this example, and consider only the dog, the example approximates what have often been called "natural signs," as when a dark cloud is a sign of rain. It is in this way that we wish the experiment to be considered.

The second example is drawn from human behavior. A person on 
the way to a certain town is driving along a road; he is stopped by another person who says that the road is blocked some distance away by a landslide. The person who hears the sounds which are uttered does not continue to the point in question, but turns off on a side-road and takes another route to his destination. It would be commonly said that the sounds made by the one person and heard by the other (and indeed by the utterer also) were signs to both of them of the obstacle on the road, and in particular were language signs, even though the actual responses of the two persons are very different.

Common to these two situations is the fact that both the dog and the person addressed behave in a way which satisfies a need-hunger in the one case, arrival at a certain town in the other. In each case the organisms have various ways of attaining their goals: when food is smelled the dog reacts differently than when the buzzer is sounded; when the obstacle is encountered the man reacts differently than when spoken to at a distance from the obstacle. Further, the buzzer is not responded to as food nor the spoken words as an obstacle; the dog may wait awhile before going for food and the man may continue to drive for a time dowit the blocked road before turning off to another road. And yet in some sense both the buzzer and the words control or direct the course of behavior toward a goal in a way similar to (though not identical with) the control which would be exercised by the food or the obstacle if these were present as stimuli: the buzzer determines the dog's behavior to be that of seeking food in a certain place at a certain time; the words determine the man's behavior to be that of getting to a certain town by avoiding a certain obstacle at a given place on a given road. The buzzer and the words are in some sense "substitutes" in the control of behavior for the control over behavior which would be exercised by what they signify if this was itself observed. The differences between non-language and language signs remain for subsequent discussion.

It is clear at once that the formulation of 'sign' frequent in early behavior theory is too simple: namely, it cannot be simply said that a sign is a substitute stimulus which calls out to itself the same response which would have been called out by something else had it been present. 
For the response to food is to food itself, while the response to the buzzer is not to it as if it were food; and the actual response to the situation in which the sign appears may be greatly different from the response to a situation where what is signified, and not the sign, is present. The dog, for instance, may salivate when the buzzer is sounded but it cannot actually eat unless food is present; the man may feel anxiety when he is addressed, but his turning off the road before reaching the obstacle is a very different response from that which he would make if he had gone directly to the place of blockage itself (and even more different from the behavior of the person who told him of the obstacle.

Nor can the difficulties in the earlier attempts to identify signs with any and all substitute stimuli be avoided by attempting to say that whatever influences a response with respect to what is not at the moment a stimulus is a sign. For example, a drug will influence the way an organism will respond to stimuli which later affect it, and yet it would be too great a departure from common usage to call such a drug a sign.

The difficulties in these formulations may perhaps be avoided if, as our examples suggest, signs are identified within goal-seeking behavior. So in the light of our analysis of what the two examples chosen as a point of reference have in common (and neglecting for the time being their differences) we arrive at the following preliminary formulation of at least one set of conditions under which something may be called a sign: If something, $A$, controls behavior towards a goal in a way similar to (but not necessarily identical with) the way something else, $B$, would control behavior with respect to that goal in a situation in which it were observed, then $A$ is a sign.

The buzzer and the spoken sounds are then signs of food and obstacle because they control the course of behavior with respect to the goals of getting food and getting to a certain place in a way similar to the control which food and obstacle would exercise if they were observed. Whatever exercises this type of control in goal-seeking behavior is a sign. And goal-seeking behavior in which signs exercise control may be called sign-behavior. 


\section{Toward Precision in the Identification of Sign-Behavior}

For many purposes the preceding account of a sign is adequate; it at least suggests a behavioral way of formulating what is commonly meant in saying that a sign "stands for" or "represents" something other than itself. But for more strictly scientific purposes a more exact formulation is required in order to clarify the notions of similarity of behavior and goal-seeking behavior. We might at this point simply leave it to the scientists in their field to state further refinements, and indeed anything we add is in the nature of the case tentative. But since our concern is to push semiotic as rapidly as possible in the direction of a natural science, the following suggestions are made.

Implicit in the preceding account are four concepts which need further clarification: preparatory-stimulus, disposition to respond, responsesequence, and behavior-family. When these notions are elucidated a more precise statement of a set of conditions sufficient for something to be called a sign can be given.

A preparatory-stimulus is any stimulus which influences a response to some other stimulus. Thus it has been found by O. H. Mowrer that the magnitude of the jump of a rat to a shock stimulus is increased if a tone sounds before the shock stimulus is encountered. ${ }^{\mathbf{D}}$ Such a stimulus differs from other stimuli, say the shock, in that as a preparatorystimulus it influences a response to something other than itself rather than causing a response to itself (it may of course also cause a response to itself, that is, not be merely or solely a preparatory-stimulus). By a stimulus is meant, following Clark L. Hull, any physical energy which acts upon a receptor of a living organism; the source of this energy will be called the stimulus-object. By a response is meant any action of a muscle or gland; hence there are reactions of an organism which are not necessarily responses. A preparatory-stimulus affects or causes a reaction in an organism, but, as Mowrer makes clear, it need not call out a response to itself, but only to some other stimulus. In the account toward which we are moving it is not held that all preparatory-stimuli are signs, but only that preparatory-stimuli which meet certain additional requirements are signs. That a preparatory-stimulus need not when presented call out a response makes intelligible the fact that a 
command to turn right at a certain place may produce at the time of utterance no overt, or as far as we know, "implicit" response of turning right, and yet may determine that the person commanded turns right when he reaches the place in question. A preparatory-stimulus does however cause some reaction in an organism, affects it in some way, and this leads to the introduction of the term 'disposition to respond.'

A disposition to respond to something in a certain way is a state of an organism at a given time which is such that under certain additional conditions the response in question takes place. These additional conditions may be very complex. An animal disposed to go to a certain place to obtain food may not go there even if food is observed-he may not be willing or able to swim across an intervening water barrier or to move if certain other animals are present as stimulus-objects. The complex of conditions also includes other states of the organism. The person commanded to turn at a certain corner may not turn even when the corner is reached: as he walked to the corner he may have come to believe that his informant was deliberately trying to misdirect him, so that confidence in one's informant may be at times a necessary condition for making a response to which one is disposed because of signs.

There may be dispositions to respond which are not caused by preparatory-stimuli, but every preparatory-stimulus causes a disposition to respond in a certain way to something else. Logically, therefore, 'disposition to respond' is the more basic notion, and a preparatorystimulus is a stimulus which causes a disposition to respond in a certain way to something else. And since not all preparatory-stimuli would normally be called signs, and not all dispositions to response which are caused by preparatory-stimuli are relevant to the delimitation of signprocesses, additional criteria are involved; and to be in accord with our own preliminary formulation of sign-behavior, these criteria must introduce the notion of behavior toward a goal.

A response-sequence is any sequence of consecutive responses whose first member is initiated by a stimulus-object and whose last member is a response to this stimulus-object as a goal-object, that is, to an object which partially or completely removes the state of the organism (the "need") which motivates the sequence of responses. Thus the series of responses of a hungry dog which sees a rabbit, runs after it, kills it, 
and so obtains food is a response-sequence. For the sight of the rabbit starts a series of responses to the rabbit in terms of which the rabbit is finally obtained as food. The intervening responses in the sequence can occur only if the environment provides the necessary stimuli for their release, and such sources of stimuli may be called supporting stimulus-objects. The terrain over which the dog runs in this case provides the support necessary for the responses of following the rabbit and tracking it down, while the rabbit provides the stimuli initiating and terminating the series of responses.

A behavior-family is any set of response-sequences which are initiated by similar stimulus-objects and which terminate in these objects as similar goal-objects for similar needs. ${ }^{E}$ Hence all the responsesequences which start from rabbits and eventuate in securing rabbits as food would constitute the rabbit-food behavior-family. A behaviorfamily may in an extreme case have only one member; no limit is set to the number of possible members. Behavior-families have various degrees of inclusiveness. All the objects which a dog eats would, for instance, determine an extensive "object-food" behavior-family which would include the rabbit-food behavior-family as a subordinate behavior-family.

In these terms it is possible to formulate more precisely a set of conditions sufficient for something to be a sign: If anything, $A$, is a preparatory-stimulus which in the absence of stimulus-objects initiating response-sequences of a certain behavior-family causes a disposition in some organism to respond under certain conditions by response-sequences of this behavior-family, then $A$ is a sign.

According to these conditions, the buzzer is a sign to the dog since it disposes the animal to seek food in a certain place in the absence of direct stimulation from food objects at this place, and similarly, the spoken words are signs to the driver since they dispose him to responsesequences of avoiding an obstacle at a certain point on a certain road although the obstacle is not itself at the time of hearing the sounds a stimulus-object.

The merit of this formulation is that it does not require that the dog or the driver respond to the sign itself, the sign serving merely as a preparatory-stimulus for response to something else. Nor does it require 
that the dog or the driver finally respond overtly as they would if food or an obstacle had been stimulus-objects; it merely requires that if the animal makes the response-sequences which it is disposed to make when certain additional conditions are met (conditions of need and of supporting stimulus-objects) these response-sequences will be of the same behavior-family as those which the food or obstacle would have initiated. In this way the difficulties which earlier behavioral formulations of signs encountered are avoided. And yet objective behavioral criteria are furnished for determining whether something is or is not a sign. It is further believed that these criteria do not deviate from those which underlie certain common usages of the term 'sign.'

\section{Some Objections Considered}

By this time a mixed feeling of dismay, fear, anger, and resentment may well have overcome readers of contemporary literature on "semantics." Semiotic is not merely losing its entertaining character, they may say, but is slipping into the abyss of technicalities and hard work. And so it is! And so it must if the purpose be scientific. For science has always in its advance forced us away from the presented surface of familiar things to the laborious discovery of those properties of things which give insight, predictions, and control of this surface. And there is no reason why the scientific advance of semiotic should shun this road. No reason why sign-processes, for all their immediate sense of familiarity, should not be as complex as any chemical structure or biological functioning. And we have already admitted that for other immediate purposes, other less technical analyses may be more useful.

There is, however, a genuine question as to whether the present approach loses its relevance for the wide range of problems which draw the attention of the contemporary world to signs. And there is a real danger here because of the fact that we are certainly not at present able to analyze in precise behavioral terms the more complex phenomena of esthetic, religious, political, or mathematical signs, or even the common language of our daily existence. But later chapters will attempt to show that the present approach has, even now, many suggestions to offer in these fields, and that because of a consideration 
soon to be mentioned, the detailed analysis of signs and their significations in terms of behavior is not always necessary since we can, within limits, accept the results of analyses which have already been obtained in these fields and even verbal reports by a person on his own signs.

An objection frequently raised to the preceding type of behavioral formulation may be expressed as follows: "The observation of response-sequences is at best one test for the existence of signs, and one in fact seldom employed. Take a person reading a book alone in his room, say a book about Alaska; certainly the marks on the pages of this book are signs to the reader at the moment he reads them. And he knows this, and knows what they signify, quite independently of how he would actually respond to Alaska if he were there, and independently in fact of any response which he makes to his environment. This suggests that there is something essentially wrong with a behavioral formulation. Behavior is at times evidence of signs, but there may be other and better and more available evidence - such as that given by selfobservation."

Now much of this contention may be admitted: there certainly may be other evidence for sign-processes than the actual observance of response-sequences, and self-observation may supply this evidence. But to admit these points does not show an inadequacy in the behavioral formulation.

The situation may be clarified in this way. The preceding formulation of 'sign' is not a definition in the sense that it gives the necessary and sufficient conditions for something to be a sign. It does not say that something is a sign if and only if the conditions laid down are met, but merely that if these conditions are met, then whatever meets them is a sign. ${ }^{G}$ There may be various kinds and degrees of evidence that these conditions are in fact met. And it is perfectly possible for there to be other sets of conditions proposed for calling something a sign, and these are acceptable to the present behavioral formulation if they are such that there is a constant connection between the two sets of sufficient conditions.

The central methodological issues may be analyzed as follows. The set of conditions which has been proposed as sufficient for calling some- 
thing a sign did not stipulate that the organism for which it is a sign actually performs response-sequences of a given behavior-family, but merely that it be disposed to perform them, that is, would perform them under certain conditions. The question then is, what kind of evidence permits us to say that an organism is disposed so to act.

The most complete evidence is of course to make available the conditions in question and then see whether response-sequences of the given behavior-family actually occur. If one wants to know whether the buzzer is a sign to the dog of food in a certain place, one takes a hungry dog and investigates whether the buzzer is the cause of responsesequences of seeking food in that place when the food itself is not at the time a stimulus-object. If the dog under these conditions goes to the place in question and eats the food there available, then one has shown that the dog was disposed by the buzzer to responses of the same behavior-family that are called out by the presence of food as a stimulusobject at that place.

But there are other possibilities for showing the existence of such a disposition. If we define a segment of a response-sequence as any series of consecutive responses within a response-sequence which starts from an initial member but does not contain the final member, then we may often observe that an animal performs such a segment of a responsesequence though it does not perform the whole sequence. If food is not made available to the animal it cannot of course eat food, but it may be observed to go toward the place in question and to salivate or otherwise react in ways characteristic of seeking and eating food. Such data may then be taken as evidence that the dog is disposed to behavior of the kind required by the formulation of a sign, and such evidence is reliable in proportion to the degree to which it is distinctive of the behavior-family in question.

The evidence required may not even be a segment of a responsesequence, may not in fact be a response at all. If, for instance, any state of the organism could be found-say its brain waves-which is such that when this state is present the animal later responds in the way required by the formulation of a sign, then this state of the organism would itself be a sufficient condition for saying that the preparatory-stimulus which occasioned this state was a sign. In other words, there may be 
other ways of determining the existence of a disposition to respond in a certain fashion than by directly observing the response itself.

Finally, among such ways is the possibility of using in some cases the verbal responses of an organism as evidence of whether something is or is not a sign to this organism. By correlating such verbal responses with the rest of the behavior of the organism it is possible to find out the extent to which they are reliable evidence as to the existence of signprocesses. That they are reliable to some extent is not in doubt, and so semiotic can make use of them as evidence for the existence of signs, and indeed must in its present state make as much use of them as it can. But since signs can occur without verbal responses, and since such responses are not always reliable evidence, they are at best evidence for the existence of signs and not either necessary or sufficient conditions for the occurrence of signs. The science of signs must deal with animals and children and insane persons that cannot report on their behavior, as well as with persons whose reports are often unreliable, so it must start with criteria for the existence of sign-processes which are applicable in these cases. Hence the contention that the behavioral formulation is the primary one with which other sets of conditions are to be correlated as alternative sets of sufficient conditions or merely as evidence that a specific set of conditions is fulfilled.

The book on Alaska read alone may then be said to be to the reader a complex of signs prior to the observation of his overt behavior. But to know this is merely to say that there is some evidence-neural, physiological, verbal-that the man is in a state which has been found empirically to be correlated with observable sign-behavior. That he is now ready to respond in a certain way at a later time can in the last analysis only be determined by correlating his present state with how he actually responds at a later time to a given environment.

It may help to point out that a similar situation is frequent in science and in daily life. A scientist may begin a study of magnetic phenomena by starting with cases in which bodies of a certain kind are found to approach a given body, and in virtue of this fact he calls the latter body a magnet. If now he finds that all magnets have certain other properties, he may accept these properties as sufficient conditions for saying that a given body is a magnet, or at least as evidence that it 
is a magnet. The situation is akin to saying that a person is angry with someone even before he behaves angrily to that person, or that a person has typhoid fever even before the grosser symptoms which originally identified the disease appear. In all such cases a disposition to a certain later kind of action is attributed to an object or person before the action itself appears because of an empirically found correlation between some earlier state of the object or person and its later action. To say that something is a sign before responses actually take place is a precisely parallel case.

\section{Further Remarks on Sign-Processes}

The behavioral formulation of sign-behavior which has been proposed has certain consequences which are worth noting. ${ }^{\mathrm{H}}$ It avoids, in the first place, the frequent misinterpretation that a sign must itself be a response. Responses may, of course, under certain circumstances be signs but they need not be, and signs need not be responses. For while every sign-process involves a disposition to respond, the sign itself may be any feature of any stimulus-object which acts in the above manner as a preparatory-stimulus; such stimuli are not limited to responses, and only when a response is itself a stimulus of this sort is it a sign.

This approach also avoids the error of those accounts of signs which neglect their relation to the situation in which behavior occurs. Such accounts often imply that the organism responds to the sign alone. But the fact that behavior takes place within a supporting environment implies that the sign alone does not cause the response evoked, since the sign is merely one condition for a response-sequence in the given situation in which it is a sign. The dog upon hearing the buzzer does not seek food wherever it happens to be (though certain components of a food-response-such as salivation-may appear when the buzzer is heard); only if a supporting environment is present will it seek food. If the situation does not support certain responses, then no complete response-sequence of a food behavior-family can take place.

For something to be a sign to an organism requires that the organism be influenced, in the way described, by the presence of that something; 
it does not require that the organism signify that the something in question is a sign, for a sign can exist without there being a sign that it is a sign. There can, of course, be signs that something is a sign, and it is possible to signify by some signs what another sign signifies. But this need not take place, and the temptation to attribute such complications to animal, or even to all human, sign phenomena must be constantly avoided.

The proposed formulation also avoids the ambiguity of the frequent attempts to define signs in terms of "appropriate behavior." It is true that the response-sequences prepared by the buzzer are "appropriate" to the goal of securing food in the sense that they are responsesequences of a food behavior-family. It is further true that the buzzer normally will not remain a sign of food if the responses to situations in which buzzers sound do not continue to meet a need, that is, do not obtain food. And yet the question of whether a response-sequence initiated in a situation does in fact reach a certain goal does not enter into the formulation of 'sign' itself. The buzzer is a sign if it meets the criteria proposed even if the situation immediately confronting the animal is such that going to the box is inappropriate to the goal of obtaining food, as it would be if such action did not secure food. Since it is obvious that sign-behavior does not in all cases eventuate in appropriate (that is, goal-attaining) responses, it is proper that the general account of signs should not involve the question of the appropriateness of the responses which occur in the situation in which the sign operates.

The proposed formulation also helps to resolve the ambiguities in the various "context" theories of signs. It is true that a sign can be described only by reference to the specific way it functions in specific situations. But since the situation in which the sign appears is generally a very different situation from that in which it does not appear, it is somewhat misleading to suggest that a sign signifies the missing part of a context in which it formerly appeared. The fact that a sign functions as a substitute for an absent something in the control of behavior keeps the "substitutional" emphasis of the context formulations without suggesting that the situations in which the sign does and does not appear are otherwise identical.

Finally, the present account does not depend upon a decision as to 
how such terms as 'habit,' 'substitute stimulus,' and 'conditioned response' are to be used. It tries to isolate a certain kind of behavior, sign-behavior (that is, behavior in which signs occur); the relations of sign-behavior to unlearned behavior, habits, substitute stimuli, conditioned responses, implicit responses, antedating responses, pure-stimulus acts, and the like are then further questions to be dealt with by students of the general theory of behavior.

\section{The Basic Terms of Semiotic}

It is now possible to isolate the basic terms of semiotic, for such terms will simply refer to various discernible aspects of sign-behavior. The term sign has already been introduced, or to speak more exactly, a criterion has been given under which certain things are admitted to be signs-whether in the course of our inquiry other criteria will have to be given to isolate other classes of signs remains for the time being an open question. But we at least are able to say that if something is a preparatory-stimulus of the kind specified in our previous formulation it is a sign. And this is the necessary first step for building a science of signs. For it identifies a subject matter for such a science and permits the introduction of a number of other terms for talking about this subject matter. Such terms can be introduced in a number of ways; we propose the following method of building a language to talk about signs.

Any organism for which something is a sign will be called an interpreter. The disposition in an interpreter to respond, because of the sign, by response-sequences of some behavior-family will be called an interpretant. Anything which would permit the completion of the response-sequences to which the interpreter is disposed because of a sign will be called a denotatum of the sign. A sign will be said to denote a denotatum. Those conditions which are such that whatever fulfills them is a denotatum will be called a significatum of the sign. A sign will be said to signify a significatum; the phrase "to have signification" may be taken as synonymous with "to signify."

So in the example of the dog, the buzzer is the sign; the dog is the interpreter; the disposition to seek food at a certain place, when caused 
by the buzzer, is the interpretant; food in the place sought which permits the completion of the response-sequences to which the dog is disposed is a denotatum and is denoted by the buzzer; the condition of being an edible object (perhaps of a certain sort) in a given place is the significatum of the buzzer and is what the buzzer signifies.

In the case of the driver the words spoken to him are signs; the driver is the interpreter; his disposition to respond by avoiding a landslide at a certain place in the road is the interpretant; the landslide at that place is the denotatum; the conditions of being a landslide at that place is the significatum of the spoken words.

According to this usage of terms, while a sign must signify, it may or may not denote. The buzzer can signify to the dog food at a given place without there being food at the place in question, and the landslide signified by the spoken words may not in fact exist. Usually we start with signs which denote and then attempt to formulate the significatum of a sign by observing the properties of denotata. But it is possible at the higher levels of human sign-behavior to determine by decision the significatum of a sign (to "lay down" the conditions under which the sign will denote), and in this case the problem is not what the sign signifies but whether or not it denotes anything. We encounter cases of this nature frequently in the more complex sign-processes.

The relation between interpretant and significatum is worth noting. The interpretant, as a disposition to respond caused by a sign, answers to the behavorial side of the behavior-environment complex; the significatum, as the set of terminal conditions uncier which the responsesequences to which the organism is disposed can be completed, connects with the environmental side of the complex. Each therefore involves the other. A complete description of the interpretant would include a description of what the organism is disposed to act toward, and a formulation of the significatum is simply a formulation of what would permit the completion of the response to which the organism is disposed because of a sign. The distinction between behavior and environment need not of course coincide with the distinction between the organism and the non-organic world, since an organism can respond to other organisms and to itself. It is because of this that parts of the organism, dreams, feelings, and even interpretants can be signified. 
Under the present usage, a sign cannot signify or denote its own interpretant, though it may signify and denote the interpretants of other signs (as in the case of 'interpretant' itself). The buzzer does not signify the dog's disposition to respond but signifies food at a given place and time. This usage is not the only possible one, and at no point is the language of semioticians more at variance. A merit of the present usage of 'signify' is that it does not make the significatum of every term-such as 'spiral nebula' or 'atom'-include biological events, though it recognizes that there are no signs which signify without dispositions to respond (that is, without interpretants). And since with this usage a sign does not denote its significatum, the temptation is avoided to make the significatum into a special kind of thing-a temptation which seems to underlie the Platonic doctrine of Ideas and various philosophic doctrines of "subsistence."

The term 'meaning' is not here included among the basic terms of semiotic. This term, useful enough at the level of everyday analysis, does not have the precision necessary for scientific analysis. Accounts of meaning usually throw a handful of putty at the target of sign phenomena, while a technical semiotic must provide us with words which are sharpened arrows. 'Meaning' signifies any and all phases of sign-processes (the status of being a sign, the interpretant, the fact of denoting, the significatum), and frequently suggests mental and valuational processes as well; hence it is desirable for semiotic to dispense with the term and to introduce special terms for the various factors which 'meaning' fails to discriminate.

Since something is a sign, significatum, denotatum, interpreter, or interpretant only with respect to its occurrence in sign-behavior, and since such constituents of sign-processes are studied by other sciences in other connections, the basic terms of semiotic are statable in terms drawn from the biological and physical sciences-a point which will prove to be of central significance for understanding the relation of socio-humanistic studies to the natural sciences. Since the factors operative in sign-processes are all either stimulus-objects or organic dispositions or actual responses, the basic terms of semiotic are all formulable in terms applicable to behavior as it occurs in an environment. Semiotic thus becomes a part of the empirical science of behavior, and can uti- 
lize whatever principles and predictions the general theory of behavior has attained or can attain.

The formulation in terms of other signs of what a sign signifies (the description of the conditions which something must fulfill to be a denotatum of the sign) will be called a formulated significatum. A formulated significatum is designative if it formulates the significatum of an existing sign, and is prescriptive if it formulates the significatum which a sign is henceforth to have-a distinction which the commonly employed term 'semantic rule' fails to make. A sign may, of course, signify without there being a formulation of what it signifies. A recognition of this simple fact cuts the ground out from under the frequent insinuations that a sign is "without meaning" to a person or animal who cannot "tell what the sign means." For something to signify is a different thing from the often very difficult task of formulating what it signifies.

\section{Extension of Terminology}

We now introduce a number of distinctions which in one way or another have long been current in discussion of signs, but since these distinctions are formulable in our basic terms they are now given the empirical foundation which they have customarily lacked. ${ }^{\mathbf{I}}$

A particular physical event-such as a given sound or mark or movement-which is a sign will be called a sign-vehicle. A set of similar sign-vehicles which for a given interpreter have the same significata will be called a sign-family. ${ }^{\mathrm{J}}$ A particular sound of the buzzer is, when a sign to the dog of food at a particular place, a sign-vehicle, while the set of similar sounds which at different times signify to the dog food at that place constitute the sign-family of which each particular buzzer sound is a member. If the dog were trained so that lights signified food at the given place, a given light would have the same signification as a given sound, but the sounds and the lights would belong to different sign-families. Often the distinction of sign-vehicle and sign-family is not relevant, so that we shall frequently speak of signs without further qualification; but the distinction is of theoretical importance and we must be in a position to invoke it when necessary. 
A sign-vehicle that does not belong to a sign-family is a unisituational sign since it has signification in only one situation. Such signs rarely if ever occur; most signs are plurisituational.

To the degree that a sign has the same signification to a number of interpreters it is an interpersonal sign; to the degree that this is not so the sign is a personal sign. The interpreters for whom a sign is interpersonal may be called an interpreter-family. A given sign may be in principle entirely interpersonal or entirely personal; most signs are neither. Since it is always possible in principle to find out what a sign signifies for a given interpreter, and so make it interpersonal, no sign is inherently personal; but in actual practice many signs are highly personal-the signs of the schizophrenic provide extreme examples. It may be remarked that we should not necessarily classify a note which a person writes to himself for reading at a later time as interpersonal; such a note would be personal by the criterion proposed if the signs were signs to him alone, and interpersonal if this were not the case even though no one else read the note.

A sign is vague to a given interpreter to the degree that its signification does not permit the determination of whether something is or is not a denotatum; to the extent that a sign is not vague it is precise. ${ }^{\mathbf{K}}$ Vagueness shows itself behaviorally in an uncertain and hesitant response to an object to which the organism has been directed by a sign. At the human level vagueness can be quantified by asking the interpreter whether given objects or situations are denoted by a given sign, and noting those of which he is unsure. Vagueness is interpersonal to the degree that a sign is vague in the same way to different interpreters. Thus 'consciousness' may be vaguer than 'life' to one individual and not to another; but it may also be interpersonally vague among those who speak English. The precision of terms may always be increased where that is desirable, but absolute precision can never be attained. Nevertheless the present account of vagueness is undoubtedly vaguer than it need be!

A sign-vehicle is unambiguous when it has only one significatum (that is, belongs to only one sign-family); otherwise ambiguous. 'Chair' is an example in English of an ambiguous sign; if we know only that a person "holds a chair," we do not know whether he is grasp- 
ing an object one can sit on or has a certain kind of academic appointment. A sign in the sense of a sign-family cannot be ambiguous for by definition all the members of a sign-family have the same significatum; since in what follows we are usually concerned with sign-families we can use 'significatum' instead of 'significata' when talking about a sign.

A sign is singular when its significatum permits only one denotatum; otherwise it is general. 'The 1944 President of the United States' is a singular sign, since it could not, because of its signification, denote more than one person. So is the sign 'I,' for though this sign is a family of sign-vehicles each member of which has the same significatum, the significatum is such that in each instance of signifying there can only be one denotatum (the person who produces the sign-vehicle of that instance). 'House' is by contrast a general sign since its significatum does not limit the possible denotata of its sign-vehicles to one. Degree of generality is a matter of the interrelationship of significata. 'Colored' is more general than 'red' since the conditions for something being a denotatum of 'red' are sufficient to insure that that something meets the conditions for being a denotatum of 'colored,' while the significatum of 'colored' is such that it can denote something without 'red' denoting it. A sign which is more general than another sign, or of equal generality with it, is an analytic implicate of the other sign; in this sense 'colored' is an implicate of 'red' (later we will add the concept of contradictory implicate). The extreme of generality is reached when the significatum of a sign is such that the sign denotes the denotata of any other sign. Such a sign is universal, and is an implicate of every sign; the terms 'being' and 'entity' seem to be, in the vocabulary of some philosophers, universal signs.

A sign is synonymous with another sign if the two signs belong to, or are, different sign-families and yet have the same significatum. Signs are similar in signification to various degrees; hence it would be possible to make synonymity a matter of degree. But since definitions and what we shall call symbols illustrate the limit of this process-that is, are instances of identity of signification-it seems wise to limit synonymity to cases in which the signs have the same significata. If synonymity is thus limited, it must be recognized that signs that are 
often called "synonyms" are simply signs with highly similar significata.

A sign is reliable to the degree that the members of the sign-family to which it belongs denote; otherwise unreliable. The degree of reliability (and so the degree of unreliability) of a sign is capable of quantitative formulation. If the dog obtained food 90 per cent of the times the buzzer sounded, the buzzer sign is 90 per cent reliable; such a statement would not of course insure that the degree of reliability of the sign would continue unchanged in the future. A sign is iconic to the extent to which it itself has the properties of its denotata; otherwise it is non-iconic. A portrait of a person is to a considerable extent iconic, but is not completely so since the painted canvas does not have the texture of the skin, or the capacities for speech and motion, which the person portrayed has. The motion picture is more iconic, but again not completely so. A completely iconic sign would always denote, since it would itself be a denotatum. A sign which is to some extent iconic may itself have properties which are not iconic and which are not relevant to its signification. One of the dangers of the use of models in science, for instance, arises out of the temptation to ascribe to the subject matter of a theory properties of the model illustrating the theory which are not involved in the theory itself. ${ }^{\mathrm{L}}$

The terms which have been introduced in this section constitute only a portion of the terms which students of signs must employ. While a more precise formulation of these terms can no doubt be given, and would be desirable, the present analysis may serve to show that a behavioral formulation of the basic terms of semiotic provides a foundation for the introduction of such other terms as the science of signs finds necessary. This suggests that a behavioral semiotic is comprehensive enough to incorporate the distinctions now current in sign-analysis. The fear that such an approach might be too simple to deal with the complexities of sign phenomena is in this way mitigated.

\section{Signal and Symbol}

Various other distinctions between signs will appear throughout our discussion, but there is one basic distinction in the literature of 
semiotic which warrants consideration now: the difference between signal and symbol. Later we will discuss the way logicians and philosophers have tried to make the distinction in question; for the moment we will begin with the distinction as it has presented itself to students of behavior. A frequent terminology for the difference is 'sign' and 'symbol,' but since we regard both the distinguished items as signs, the terminology chosen will be 'signal' and 'symbol.' $M$

Robert M. Yerkes remarks as follows" : "Not rarely sign and symbol are used as synonymous. I wish to propose differing meanings. . . Whereas the sign is an experience-act which implies and requires as its justification in terms of utility a succeeding experience-act, the symbol has no such implication and is an experience-act which represents or may function instead of whatever is represented. The sign sooner or later loses its meaning apart from its context; the symbol does not. The sign is not a substitute for the original experience-act, whereas the symbol may be." The buzzer in our example of the dog would presumably be regarded by Yerkes and certain other students of animal behavior as a sign (that is, signal), whereas if the buzzer stopped sounding before the animal was allowed to seek food (that is, if its response were delayed) such persons would seem to be saying that something else must in the interval act as a substitute for the buzzer if food behavior is later to be called out, and such a substitute would be a symbol. This distinction was made by W. H. Hunter to explain the results of his experiments on delayed reactions.

While it is highly doubtful that all delayed responses need explanation by the introduction of a special class of signs (since a delay in response is compatible with our general description of signs), there is no doubt but that Yerkes is calling attention to differences in signprocesses which are behaviorally recognizable and deserve a name. Suppose that the buzzer produced in the dog some response which then functioned as a sign for food at the given place without the buzzer sounding, then such a "response-sign" would have certain distinctive characteristics in virtue of the fact that it is relatively independent of the environment and is a substitute for another sign with which it is

${ }^{2}$ Chimpanzes, a Laboratory Colony, p. 177. 
synonymous. At the language level examples are more easily recognizable. If the driver in the car had been told to turn to the right at the third intersection, he might have held up three fingers of his right hand until he reached the intersection in question, or might have continued repeating the instructions to himself; such action on his part would be a sign to him signifying what the original spoken words signified, and such a sign would guide his behavior in the absence of the spoken signs.

Generalization from such examples suggests the following distinction: Where an organism provides itself with a sign which is a substitute in the control of its behavior for another sign, signifying what the sign for which it is a substitute signifies, then this sign is a symbol, and the sign-process is a symbol-process; where this is not the case the sign is a signal, and the sign-process is a signal-process. More succinctly, a symbol is a sign produced by its interpreter which acts as a substitute for some other sign with which it is synonymous; all signs not symbols are signals.

The advantage of such symbols is found in the fact that they may occur in the absence of signals provided by the environment; an action or state of the interpreter itself becomes (or produces) a sign guiding behavior with respect to the environment. So if a symbol operates in the dog's behavior, the symbol could take the place in the control of behavior which the buzzer formerly exercised: hunger cramps for instance might themselves come to be a sign (that is, a symbol) of food at the customary place. The relative independence of such signs from signals provided by the environment has, however, as its reverse pole certain disadvantages: symbols are especially unreliable. In the experiment the conditions of the environment were such that food normally was found at a certain place when the buzzer sounded, but not otherwise; it is more unlikely that the environment would be such that food would be in the given place when and only when the animal was hungry. This suggests that the connection between symbols and signals is more intimate than Yerkes' words imply; while it is true that the symbol may perhaps persist as a sign longer than the signal which is not accompanied or followed by a denotatum, the fact that a symbol is ultimately a substitute for a signal means that it too is normally 
subject in its genesis and its continuance upon its capacity to lead to behavior which on the whole attains goal-objects.

The recognition that a symbol requires an action or state of the organism which provides a substitute sign synonymous with another sign avoids a number of possible confusions. Not all states or actions of an organism which are signs or produce signs are thereby symbols. A person may interpret his pulse as a sign of his heart condition or certain sensations as a sign that he needs food; such signs are simply signals; his resulting words-when substitutes for such signals-would however be symbols. Nor are all sounds uttered by a person or by others symbols even when they are signs: sounds too may be simply signals. Further, the symbol need not itself be an action or state of the organism though it is produced by such an action or state: the written record of a conversation, as a substitute sign for spoken words, is still a symbol though it exists, as written, in the physical environment of the organism. Finally, not all signs produced by an organism by its action on the environment are symbols: the cat which pushes a button which lights a bulb and thus provides itself with the customary sign of food has produced a signal but not necessarily a symbol since the light need not itself be a substitute for another sign. ${ }^{N}$

Doubt may be raised whether the term 'symbol' should be used for the kind of sign-behavior which we have isolated. It may be objected that religious symbols, such as a cross, or literary symbols, such as a "golden bowl" symbolizing life, or social symbols, such as representations of a totemic animal, are not symbols in the sense defined. It must be granted that the proposed use of the term is at variance with some common usages, but we have seen that a terminology for semiotic can be expected to show such variance. In the cases in question, however, it is doubtful if the disagreement is in fact great. The cross and the figure of the totemic animal are to be sure iconic, but iconic symbols are admissible on our account as one subclass of symbols. And a particular cross or a particular carved animal may well be substitutes for objects that were themselves signs-the original cross signifying (at the minimum) the crucifixion of Christ and the living animals signifying something about the persons for whom they were signs. The words 'golden bowl,' as a literary symbol for the fragility of life, 
are even more clearly substitutes for an actual or imagined bowl, delicate and beautiful but with a fatal flaw, which to Henry James had already taken on the status of an iconic sign; the words themselves are in this case not iconic. So the proposed usage does not in fact seem to violate religious, anthropological, and literary usages. While a symbol may be iconic it is not always required to be such even in nontechnical usages, but it is very commonly regarded as something which is a substitute for other signs and producible by its interpreters.

A more positive argument for allowing 'symbol' to mark one pole of the intended distinction can be given in virtue of the claim often made that symbols are more "autonomous" and "conventional" than signals. The proposed usage embodies and explicates this claim. The symbol is "autonomous" in the sense already discussed: since it is produced by its interpreter it may appear under a great variety of environmental circumstances and is to this extent independent of any particular external environment. And the symbol is "conventional" in the sense that no limit is set upon the actions and states and products of the organism that may operate as synonymous signs substituting for other signs. Symbols may, and indeed do, vary widely from one individual to another or from one society to another. The possibility of this variability is explained in our account without the overtones of a "convention" where it is "decided" what is to be used as a symbol. For the distinction we have drawn involves no reference to a voluntary decision in the producer of a symbol; such decisions may of course occur in the case of symbols but they are not necessarily involved in the determination of what is a symbol.

Signs which are substitutes for synonymous signs frequently occur at the more complex levels of sign phenomena. Such signs seem to be appropriately called symbols. But whatever term be used, the distinction in question is of basic importance for the development of semiotic and for the understanding of human sign-behavior.

\section{Alternatives to a Behavioral Semiotic}

The preceding analysis may be characterized as an attempt to carry out resolutely the insight of Charles Peirce that a sign gives rise to an 
interpretant and that an interpretant is in the last analysis "a modification of a person's tendencies toward action." 3 But before proceeding further in this direction it would be well to take account of the perturbations which our account must have awakened in certain readers and to ask ourselves whether we have not neglected important alternatives for the development of semiotic.

Some readers must feel strongly that we have "left something out," and perhaps something central to a theory of signs, namely the "ideas" or "thoughts" which the sign causes in its interpreter. At a number of places in our account we will return to this issue; here we must be content to isolate the underlying problem.

It is true that the preceding account has completely and deliberately avoided all use of "mentalistic" terms in building the terminology of semiotic. And it is also true that the mentalistic approach has dominated the history of semiotic and still seems to many thinkers to be a preferable alternative to the approach here developed.

The defense of a behavioral semiotic must not be misunderstood. We have not contended, and do not believe, that such terms as 'idea,' 'thought,' 'consciousness,' and 'mind' are "meaningless." Nor have we in any sense denied that an individual can observe his feelings, or his thoughts, or his dreams, or his signs in a way not possible to other individuals. Our purpose is simply to advance semiotic as a science, and it is this purpose alone which determines what basic terms are to be accepted for building the terminology of semiotic. The issue is not between "mentalism" and "behaviorism," but is solely a methodological problem: are such terms as 'idea,' 'thought,' 'mind' more or less precise, interpersonal, and unambiguous than such terms as 'organism,' 'stimulus,' 'response-sequence' and 'disposition to response'? In choosing the latter terms we but express the belief that they are the more suitable for scientific advance.

Suppose, for instance, the mentalist should argue-as he often doesthat for something to be a sign to some interpreter it must give rise to an "idea" in his mind, must cause him to "think" of something else. On this frequent manner of speaking, the buzzer is a sign to

"Collected Papers, V, $\$ 476$. 
the dog only if it causes an idea or thought of food at a certain place. A person who approaches signs behaviorally (biologically) does not have to reply that the dog does not have ideas or thoughts, but merely to ask for the conditions under which we will affirm that the buzzer occasions an idea or thought in the dog. Unless the mentalist furnishes a criterion such that other persons may employ it to test whether the dog has or has not an idea there is no way to determine the precision, interpersonality, or unambiguity of the term. Hence there is no way to control by observation statements made about the signs of the dog; and this means that no science of signs is possible. If the mentalist does propose a criterion meeting these requirements, it will always be found, I believe, that the criterion is couched in terms of biological states or actions of the dog-though it may be claimed that these are only "evidence" that the dog has an idea. But if this is so, the only scientifically relevant part of the claim that the buzzer is a sign to the dog is the part couched in biological terms. Hence semiotic as a science gains nothing by the introduction of mentalistic terms in its primitive terms, for insofar as these terms are not synonymous with behavioral terms they prove to be scientifically irrelevant.

This outcome would not be avoided by proposing to limit semiotic to beings capable of self-observation and capable of reporting on such observations. For the problem of determining whether another person has an idea is not methodologically different from determining whether a dog has an idea. True, the other person may utter the words, "The buzzer makes me think of food at a certain place." But these words are themselves physical sounds uttered by a biological organism, and while they may rightly be used even on our approach as evidence that the buzzer is a sign, the reliability of this evidence can only be checked if we are in possession of some criterion to determine whether the buzzer is or is not a sign of food at a given place. Since the behavioral approach gives this criterion and the mentalistic approach does not, a mentalistic semiotic is not an alternative to a behavioral semiotic even at the level of human beings.

It must be repeated that this does not mean that dogs and persons do not have ideas. A reader can, if he wishes, say to himself that in all sign-behavior which we discuss there occur ideas which we do not 
and cannot mention. The only point is that if he does this he cannot also say that our account is scientifically inadequate, for terms whose reliability cannot be checked by observation cannot appear in any science, behavioral or otherwise. Whether they occur somewhere else is not a problem relevant to the scientific enterprise. Therefore we conclude that mentalism provides no alternative to a behavioral semiotic if the goal of semiotic is to become a science.

It is however possible that the behavioral approach does not in fact "leave out" anything. It may well be that the main function served by the introduction of 'idea' into the criterion for a sign was to permit us to say that something may be a sign to someone even when no overt behavior occurs. This function is served in our account by the term 'interpretant,' conceived as a disposition to respond in certain ways under certain circumstances. To the extent that this is so, 'idea' and 'interpretant' may in fact be synonymous signs. And this opens the possibility that all "mentalist" terms may turn out to be incorporable within a behavioral semiotic. Since it is hard to see how the reverse situation is possible, the behavioral approach to signs shows itself once more to be strategically strong.

Does the "phenomenological description" of sign-processes furnish another alternative to a behavioral semiotic? I think not. For in a wide sense of 'phenomenological' a behavioral semiotic is phenomenological since it includes a description of observed behavior; and a narrower use of the term (the description by an individual of his own sign-processes) is covered by our admission of self-observation, an admission which is compatible with either a behavioral or a mentalistic psychology, and so does not decide between them. A human being may for instance describe his "experience" in connection with a signhis feelings, his ideas, his expectations-and may compare in these terms the different signs which we have distinguished and will distinguish. ${ }^{\circ}$ The resulting reports are reports by an interpreter on his own sign-processes, and give us certain data about these processes which we do not possess in the case of animals unable to report the results of their self-observation. But reports of self-observation in which, say, the term 'idea' is used do not themselves determine whether 'idea' is synonymous with 'observation by an interpreter of his interpretant,' 
that is, observation of his disposition to respond in such and such a way. Some "phenomenologists" might very well report that this is the case. So a phenomenological description of sign-processes, whether in the wider or narrow sense of 'phenomenological,' does not in itself negate or provide an alternative to a behavioral semiotic. Such a semiotic is able to include reports by interpreters on their sign-behavior and yet can deal with sign-behavior in animals, children, and adults where such reports are not available or not reliable.

While we admit the right of various persons to approach signs in any way they see fit, we see no evidence that mentalism or phenomenology provide alternatives to a behavioral semiotic concerned solely with the development of an empirical science of signs. 


\section{LANGUAGE AND SOCIAL BEHAVIOR}

\section{Language as Sign Phenomenon}

\section{ITHe} HE term 'language' is highly vague and ambiguous. Beyond the very general agreement that language is a sign phenomenon social in nature, there are many disagreements as to what is necessary for signs to be language signs. ${ }^{A}$ It is helpful to begin on our search for the criteria of the language sign by considering our second example of sign-behavior: the case in which one person tells the driver of a car that the road on which he is driving is blocked at a certain point.

The first item to be noted here is that the situation is one of social behavior, that is behavior in which organisms provide other organisms with stimuli. Where two or more organisms provide each other with stimuli, the social behavior may be called reciprocal; otherwise nonreciprocal. ${ }^{\mathrm{B}}$ Behavior which is not social is individual. The behavior of both the speaker and the driver is reciprocal social behavior, for the words were spoken because of the presence of the driver and the words as spoken by the speaker were stimuli to the driver. An organism which produces a sign that is a stimulus in social behavior will be called a communicator, and an organism which interprets a sign furnished by a communicator will be called a communicatee. Reciprocal social behavior may be either co-operative, competitive, or symbiotic depending upon whether the behavior of each organism aids the other in reaching a common goal (as in two persons moving a $\log$ ), or whether the behavior of each organism hinders the other in attaining a common goal (as in two dogs fighting for the same bone), or whether the be- 
havior of each organism, though influenced by the other organism, is neither co-operative nor competitive (as when animals in eating happen to direct the attention of each other to food which exists in sufficient quantity to satisfy the hunger of both animals). The language behavior in the case in question is co-operative social behavior, since each person presumably has an interest in the driver's getting to his destination without interruption, or at least in being correctly informed as to the condition of the road ahead. The general relation of language to social behavior is one problem which semiotic must face.

Especially noteworthy is the complicated sense in which the signs in this example have a common signification, that is, are interpersonal signs. Both persons interpret the signs as designating a blockage of a given road at a given point, and each would make the same interpretation regardless of which one spoke the words-and indeed even if one of the persons spoke the words to himself. A sign which has the same signification to the organism which produces it that it has to other organisms stimulated by it will be called a comsign. A comsign is thus a special class of interpersonal signs, since not all interpersonal signs are comsigns. Each of the persons in our example is thus potentially a communicator or communicatee of the spoken words, and each is a communicatee when he is a communicator (the person giving the warning hears the words which he himself utters to the other person and they have the same or a similar signification to him and to the person addressed). Yet the actual behavior of the two persons in the given situation is very different: the communicator may remain at his place in order to warn other motorists; the driver himself moves on and takes another road to his destination. Such diversity of responses of communicator and communicatee, in spite of a common signification of signs, is another fact which an adequate account of language must explain.

There is the further question as to whether language signs are symbols. This question breaks up into two parts: are the signs producible by the organisms, and are they ultimately substitutes for signals? The spoken words are produced by the communicator, but they might seem simply to be signals to the communicatee in that in the given situation they are not produced by him. The issue is here 
largely terminological. Since in the definition of a symbol it was merely required that sign-vehicles of the given sign-family be producible by the organism (and not produced), and since the driver who hears the spoken words could produce them, and may even in this situation repeat them, it does no violence to usage to say that the spoken words are symbols to both communicator and communicatee at least with respect to the criterion of producibility. ${ }^{\mathrm{C}}$

Are such words, however, substitutes for other synonymous signs? This is a complicated issue which would involve a study of the genesis of the signs produced. They would be symbols only if for each of them there could be found some other sign, with the same significatum, for which they became substitutes. Some persons might argue that the "perception" of food or obstacle was itself a signal-process, and that language signs are always substitutes for such signals. But this raises the difficult question as to whether or not perception is to be regarded as a sign-process. ${ }^{D}$ And if it is not, it is doubtful whether language signs are always preceded by signs for which they are substitutes. Because of this doubt it seems best not to require by definition that a language sign be a symbol, but to leave this open as an empirical problem for the semiotician to face.

There is another factor to be noticed. The study of other situations in which the informer and the driver speak would show not merely that highly similar spoken words occur in these situations (so that language signs are plurisituational) but that some of the spoken words can occur in combination with words not spoken in the present situation, their place in these combinations and the varieties of the combinations being, however, subject to certain restrictions. This last fact is a somewhat unusual way of saying that a language has a "grammar" or a "syntax," that is, permits only certain combinations of signs and places certain restrictions upon the way signs can appear in these combinations. If this is taken to be a requirement for a language, it follows that a language involves a plurality of signs subject to restrictions in their combinations.

Such considerations, among many others that might be introduced, make evident the complexity of factors to be taken account of in attempting to define 'language' in terms of the categories of semiotic. 
The wide divergency of usages of the term 'language' become explicable through emphases upon various ones of these factors or upon various combinations of them. We must now attempt to frame definitions of 'language' and 'language sign'-definitions which in the nature of the case will diverge from many of the current usages of these terms.

\section{The Definition of 'Language'}

For the term 'language' the following five criteria are suggested as those to be embodied in the definition.

First, a language is composed of a plurality of signs. Second, in a language each sign has a signification common to a number of interpreters. Over and above the signification of language signs which is common to members of the interpreter-family, there may of course be differences of signification for individual interpreters, but such differences are not then regarded as linguistic. The fact that a sign is to some degree personal is compatible with the requirement that a language sign be interpersonal, for all that is required is that the signs in a language have some degree of interpersonality.

Third, the signs constituting a language must be comsigns, that is, producible by the members of the interpreter-family and have the same signification to the producers which they have to other interpreters. Comsigns are either activities of the organisms themselves (such as gestures), or the products of such activities (such as sounds, traces left on a material medium, or constructed objects). An odor, for instance, might be interpreted in the same way by a number of organisms in a given situation, and hence be interpersonal, and yet would not be a comsign. Odors would be language signs only if in addition to being interpersonal they were producible by their interpreters. ${ }^{\mathbf{E}}$

Fourth, the signs which constitute a language are plurisituational signs, that is, signs with a relative constancy of signification in every situation in which a sign of the sign-family in question appears. If the term 'odor,' for example, signified differently each time the sign occurred it would not be a sign in a language even though at a given occurrence it was interpersonal. A sign in a language is thus a sign-family and not merely a unisituational sign-vehicle. 
Fifth, the signs in a language must constitute a system of interconnected signs combinable in some ways and not in others in order to form a variety of complex sign-processes.

Uniting these requirements we reach the proposed definition of a language: a language is a set of plurisituational signs with interpersonal significata common to members of an interpreter-family, the signs being producible by members of the interpreter-family and combinable in some ways but not in others to form compound signs. Or more simply, a language is a set of plurisituational comsigns restricted in the ways in which they may be combined. If the restriction as to combination be embodied in the word 'system,' we can say that a language is a system of plurisituational comsigns. And since a sign-family is plurisituational, the simplest foundation would be that a language is a system of comsign-families. A language sign is any sign in a language.

Since the term 'language' is so vague and ambiguous in current usage, it may be doubted whether it is wise to employ the term at all in semiotic. In this connection it may be pointed out that what we have really done is to define a certain set of signs as a language and then to define a language sign as a member of this set. The signvehicle 'language' is not important for the analysis. Hence it need not be used. We propose therefore to call sign-sets of the kind in question lansign-systems, and the individual members of these systems lansigns. In what follows we are thus concerned with lansign-systems and lansigns; and while we will continue to use the terms 'language' and 'language sign,' for reasons now to be given, the reader who wishes may always replace 'language' by 'lansign-system' and 'language sign' by 'lansign,' and then use the terms 'language' and 'language sign' in any way he wishes-or discard them altogether.

\section{Consideration of the Proposed Definition}

It is believed that the preceding definition of 'language' and 'language sign' is in substantial agreement with the way these terms are employed in scientific discussions of language. But there are several points of divergence worth noting in defense and in explication of the proposed definitions. 
The clear area of agreement is with those usages which regard a language as having a vocabulary (lexicon) and a grammar (syntax). The requirement that language signs be plurisituational and form a system accounts for this agreement.

A possible divergence appears in the question whether language is on our criterion social. If the insistence that a language is social means that language signs are interpersonal, and hence presuppose a plurality of interpreters, there is again no issue, for that requirement is met by the definition. If an insistence upon the social nature of language means to assert that language arises in social behavior, this is an empirical problem which is not prejudiced by our definition. We will consider this problem shortly. But if the claim is made that what are commonly called language signs occur, after they have arisen, only in social behavior, even in the minimal sense of one organism producing stimuli for another organism, then the claim seems to be in fact doubtful. Suppose someone sitting alone in a room writes a poem which is then destroyed before it is read by anyone. In this case there is no social behavior, that is, the poem is not produced in the presence of stimulation from other organisms nor does it act as a stimulus to any other organism. Yet surely it is intelligible to ask whether the poem was written in English or French. Our definition makes this question permissible, since while English is defined with respect to an interpreter-family the definition does not require that every sign-vehicle of the sign-families in a language be a stimulus to a number of interpreters. To require by definition that a language sign always occurs in social behavior would make it necessary to say that the poem was not written in any language whatever.

The distinction between sign-vehicle and sign-family also permits an explanation of the linguists" distinction between "speech" and "language" ('parole' and 'langue'). There are no sign-families without sign-vehicles; hence there is no language, as a system of sign-families, without the production of sign-vehicles, and it is such production which constitutes a speech-act (when the language is built from sounds) or, in general, a language utterance. The signs in such an utterance can be considered simply as particular sign-vehicles or as belonging to sign-families; in the first case we speak of speech or language utter- 
ances, while in the second case we speak of language. References to language are thus always references to sign-families (whether the reference's be to phonemes, or words, or sentences, or grammatical forms). The implication that language has an existence apart from utterances is avoided on the basis of this formulation while still making it possible to distinguish statements about language utterances from statements about language.

It should now also be evident why the definition of language was not couched in terms of communication. The analysis of the term 'communication' will be delayed until in a later chapter we discuss the uses to which signs may be put. Here we need only remark that if 'communication' be restricted (as we will later do) to the arousing of common significata by the production of signs, then it follows from the definition of language that whenever a language sign produced by one organism is a stimulus to another organism in social behavior communication does occur, and such communication may be called language communication since it is produced by language signs. The relation between language and communication is then very close, though language is not defined in terms of communication. The term 'communication' itself is not limited, as we shall see, to language communication.

Such are some of the reasons why 'language' ('lansign-system') has not been defined in terms of social behavior. Its genetic relation to such behavior and its effects upon social behavior are topics for later treatment.

Finally, we must mention that many persons, especially professional linguists, will object to our omission from the definition of language the requirement that language signs be spoken sounds. On our part we see no theoretical reasons for the inclusion of such a requirement; to insist on it would be comparable to insisting that buildings made of different materials should not all be called buildings. I would propose general linguistics to name the study of languages as defined (that is, lansignsystems); we can then differentiate between auditory, visual, tactual languages, depending on the sign-vehicles which occur. Students of spoken and written languages, however, can if they wish differentiate languages as a subclass of lansign-systems and hence language signs 
as a subclass of lansigns. In this case their study, if called 'linguistics,' would be some portion of general linguistics.

\section{The Interpersonality of the Language Sign}

A sign is on the way to becoming a language sign if it is producible by organisms and has for these organisms a common signification regardless of which organism produces it. We have called such signs comsigns (comsignals or comsymbols as the case may be). Comsigns become language signs when they are subject to rules of combination with other comsigns.

As George $\mathrm{H}$. Mead saw with incisive insight, the crucial genetic problem in the origin of comsigns is to explain how an animal that does something that another animal reacts to as a sign can itself react to its own action as a sign with the same significatum. In our terms, only if an organism can react to its own activity (or its products) with an interpretation (interpretant) similar to that made to this activity (or its products) by other organisms can a sign producible by one organism have to that organism a signification in common with that of other interpreting organisms. Mead pointed out that most responses of an organism do not affect the receptors of an organism making the response as they do the receptors of other organisms--the organism, for instance, does not see its facial movements as do other organisms. And we might add, since most animals and young children do not produce many objects with their activity the stimulus which is being sought to explain similar interpretants cannot in general be looked for among physical objects produced by activity but must be found in organic responses themselves or in such products as sounds resulting from responses. If we put to one side the question whether there are unlearned comsigns, the problem of the genesis of acquired comsigns is the problem of how organisms attain similar interpretants to a stimulus which either can produce.

Mead sought for an answer to this problem of the appearance of comsigns by calling attention to the fact that the sounds made by an organism were heard by that organism as they are heard by other organisms. And this is certainly one key to the solution. For if the 
sound produced by organism $A$ became a sign to a second organism $B$, then if $B$ produced a similar sound, this sound might (by what is often called "stimulus generalization") continue to be to $B$ a sign with the same significatum that it had when produced by $A$. This is the first step. The next would be that organism $A$ must undergo the same process: the sound produced by $B$ must become to $A$ a sign with the same significatum which it had to $B$ when a similar sound was produced by organism $A$ itself; once this was gained, the sound when produced by $A$ would have the same significatum to $A$ as when produced by $B$. The sound would then have the same significatum to $A$ and $B$ whether produced by $A$ or $B$, and so would have become a comsign.

It is obvious at once that only under very unusual situations could this combination of circumstances obtain. The mere fact that an organism hears the sounds it utters as other organisms hear these sounds is at the best a necessary condition. For it is equally necessary that both organisms produce similar sounds, and that each similarly interprets the sound of the other.

That such comsigns might appear in animal behavior, and prior to language, is evident from the dog example: if two dogs were trained in the same situation, the sound of the buzzer would become to each a sign of the food at a certain place. Such signs would be interpersonal and plurisituational but not yet comsigns. If, howerer, the two animals each made a similar sound when the other organism was seeking food at a certain place, then this sound could become a sign in exactly the same way that the buzzer sound became a sign, and would have the same signification as the buzzer sound. The uttered sound would differ from the buzzer sound, however, in the fact that it was producible by the organisms and that it would be a sign to both organisms regardless of which produced it. There would be attained in this way a similar interpretant in both organisms to something produced by either organism. The uttered sound would be a comsymbol if it was a substitute for the buzzer or some other sign; otherwise it would be a comsignal.

Comsignals do not require prior signs, but a sign capable of becoming a comsymbol must be a stimulus produced by the reaction of the organism within already existing sign-behavior in which some sign has 
an interpersonal and plurisituational signification. This new stimulus which appears in the process becomes a sign in precisely the same way as the already existing sign, but must, if it is to be a comsymbol, be a stimulus which affects the receptors of the producing organism as it affects the receptors of other organisms. When this is so, the situation confers on this sign itself one of the distinctive characteristics of the language sign: it is interpreted by the producer of the sign as it is interpreted by other organisms. While it cannot be said that sounds produced by the organism are the only stimuli which can (or do) become comsymbols (or comsignals), it would seem that these are the most easily available stimuli for this purpose.

The fact that comsigns have similar interpretants (that is, dispose their interpreters to response-sequences of the same behavior-family) explains the distinctive interpersonality of the language sign. It is because of the fact that similar interpretants are aroused in the informer and the driver that we can say that the sounds uttered have the same signification to both of them. And this does not require that their actual behavior be the same in the situation, for an interpretant is a disposition to respond in a certain way under certain circumstances, and since the motivational and environmental circumstances are different for the informer and the driver the responses actually performed are different. As Mead states it, "If one calls out quickly to a person in danger, he himself is in the attitude of jumping away, though the act is not performed." 1 We obtain in this way, a behavioral formulation of the mentalists' distinction between persons "having the same idea" and yet responding differently in a given situation.

The above schematic account of the first steps in the genesis of language signs (that is, the appearance of comsigns) does not of course maintain that the requisite conditions are in fact met among nonhuman animals. Whether this is so is an empirical problem requiring detailed study of sign-behavior in such animals. But at the level of existing human society the conditions laid down as prerequisites for comsigns (and hence ultimately for language) do obtain. For the vocal responses of the child are much more numerous and variable than

${ }^{1}$ Mind, Self, and Society, p. 96. 
those of animals. And by rewarding the responses which produce sounds similar to sounds which are used by the parents, the parents train the child to produce the sounds which they use; such sounds are not necessarily signs at all, but provide excellent material for comsignals, and when fed into existing sign-processes common to parent and child, an excellent source of comsymbols. ${ }^{.}$As these sounds become increasingly plurisituational and hence producible under conditions different from the situations into which they were first introduced, and as they come to enter into stable combinatory relations with other such signs (both processes being very complex and not so far explained in the present account), they become genuine language signs. Thus would arise the highly complex signal-symbol structures characteristic of human languages. The importance of such signs for individual and social behavior will be discussed in the two concluding chapters of our study.

\section{Mead's Concept of the Significant Symbol}

The preceding account of the genesis of the language sign is in its larger outlines similar to the account given by G. H. Mead, in that both views stress the social nature of language, the dependence of language symbols upon simpler and prior signs, and the central place of sounds made by organisms in accounting for the genesis of language. But the account here given is believed to advance to some degree the analysis of the situation and to have raised a number of issues where Mead's interpretation is not entirely clear.

Mead uses the term 'significant symbol' to cover what we have called a comsign (it also seems to cover what later will be called post-language symbols). ${ }^{\text {G }}$ Mead notes that "what language seems to carry is a set of symbols answering to certain content which is measurably identical in the experience of the different individuals. If there is to be communication as such the symbol has to mean the same thing to all individuals involved." 2 The essential problem which he faced was how this common signification was attained. He sought for the source of

\footnotetext{
${ }^{2}$ Mind, Self, and Society, p. 54.
} 
significant symbols in the gesture, where a gesture was defined as the beginning of an act by one organism which serves as a stimulus for a response of another organism; thus the baring of the teeth by one organism, itself part of the act of attack, becomes a gesture when responded to by another organism as indicating what the first organism is to do. Mead's conception of the gesture is clearly related to what we have called a signal, and he himself at times calls it a non-significant symbol to distinguish it from the significant symbol. But Mead sees that such gestures are not necessarily signs to the organism which produces them, since the organism seldom responds to these gestures themselves. He notes, however, that sounds produced in the reaction may themselves serve as gestures (he calls them under these conditions "vocal gestures") and that such sounds are heard by the producing organism itself as well as by other organisms.

As it stands the account does not yet attain its intended goal: at the best it shows how a sound uttered by one organism may become to it a signal with a signification which it has for other organisms. ${ }^{\text {" }}$ But this is merely to show how gestures may have an interpersonal signification; it does not differ in essentials, for instance, from a case where a buzzer may have a common signification for two dogs; it does not in itself account for comsigns. Mead must further show how more than one organism comes to produce the sound in question (in order to get comsignals) and how sounds produced by more than one organism become substitutes to each of them for already existing interpersonal signs (in order to get comsymbols); and neither of these topics is handled in sufficient detail.

It may seem that Mead's stress upon the social act supplies the necessary principle of explanation. And so in a way it does. But his conception of the social act is not sufficiently clear. Mead, it will be recalled, at times defines a social act as one which involves "the cooperation of more than one individual, and whose object as defined by the act . . . is a social object." 3 Two people paddling a canoe in order to reach a place which each desires to reach is an example of a social act in this sense. But Mead also often speaks of social acts where two

${ }^{3}$ Mind, Self, and Society, p. 7 n. 
animals are fighting each other, and here 'social' can hardly have the sense of co-operation toward a common goal. We have seen that the term 'social' is used at times for symbiotic and competitive relations between organisms. Hence care must be taken in specifying the kind of social behavior which the comsign requires for its genesis.

Presumably Mead's position is that the significant symbol (the comsign) arises only within co-operative social acts, in which behavior toward a common goal makes possible the similarity of sounds and interpretants necessary for there to be common signification. ${ }^{4}$ So he writes: "The meaning of a gesture on the part of one organism is the adjustive response of another organism to it, as indicating the resultant of the social act it initiates, the adjustive response of the second organism being itself directed toward or related to the completion of that act." 5 Or even more explicitly: "The gesture in general, and the vocal gesture in particular, indicates some object or other within the field of social behavior, an object of common interest to all the individuals involved in the given social act thus directed toward or upon that object." 6 But if the analysis of the preceding section is correct, the comsign, while requiring minimum social behavior in the sense that organisms provide stimuli to other organisms, does not necessarily require that this act be reciprocal social behavior or co-operative social behavior-it is sufficient that the organisms perform response-sequences of the same behaviorfamily, as would be the case of two dogs each seeking food without cooperating in the process. Even if two dogs were competing for food, our analysis would permit the genesis of signs of food producible by either organism and giving rise to similar interpretants regardless of which organism made the sign in question. And even if the organisms had to co-operate to secure food, it is not a social goal which is essential but similar response-sequences (and so similar individual goals). Response-sequences of the same behavior-family are necessary to secure similar sign-vehicles and similar interpretants, but such responsesequences are possible without there being co-operative social acts.

\footnotetext{
"On p. 167 of The Philosophy of the Present, Mead, however, includes fighting among the "co-operative activities."

${ }^{5}$ Mind, Self, and Socicty, pp, 80-81.

${ }^{6}$ Ibid., p. 46.
} 
Social behavior is as genuine as individual behavior, and seems to be necessary for there to be comsigns. But it is by no means clear that comsigns can arise only in co-operative social behavior. Competitive and even symbiotic social behavior may be sufficient to account for the genesis of some comsigns. Only careful experimentation can resolve the problems which here confront us.

There is another factor in Mead's analysis that requires attention: the notion of "taking the role of the other." Speaking of the significant symbol Mead makes the following remarks: "We must indicate to ourselves not only the object but also the readiness to respond in certain ways to the object, and this indication must be made in the attitude or role of the other individual to whom it is pointed out or to whom it may be pointed out. If this is not the case it has not that common property which is involved in significance. It is through the ability to be the other at the same time that he is himself that the symbol becomes significant." 7 Now at the level of voluntary language communication it is easy to identify what Mead has in mind: there is clearly some sense in which the person who tells another that a given road is blocked is "putting himself in the place of that other" and giving information regarded as relevant to some purpose of the other individual. There is merely the question whether such phenomena are to be embodied in the definition of the language sign or are to be regarded as necessary conditions for its genesis. Mead seems to vacillate somewhat on this point: at times he talks as if role-taking were a precondition of the significant symbol and at times as if it were made possible by such symbols. ${ }^{I}$ The ambiguity is at least partially resolved if we recognize two senses of role-taking: the sense in which a person simply as a fact responds to a sound he makes as others respond, and the sense in which a person identifies the response he makes to this sound as the kind of response another person makes. Role-taking in the first sense is involved in language signs, but adds no new factor to our previous account; roletaking in the second (and more usual sense) would seem to require complex signs (and perhaps even language), since it requires the signification of another person and the attribution to that person of a

"A Behavioristic Account of thr. Significant Symbol," Journal of Philosophy, 19, 1922, p. 161. 
disposition to respond similar to that of the interpreter himself. The distinction is important since there is no evidence that taking the role of the other in the latter sense is required to explain the genesis of the language sign. Mead seems at times to avoid the difficulties in the analysis of language by invoking the notion of role-taking.

I shall not stop at this point to discuss certain problems in Mead's use of the term 'meaning' and in his occasional attempts to differentiate the significant symbol from the conditioned reflex by the introduction of "consciousness." Mead's general position needs further terminological clarification and empirical supplementation by detailed studies of signbehavior; nevertheless his treatment of the significant symbol has greatly advanced the problems in regard to language which he set for himself, and is filled with acute insights into the higher reaches of signbehavior. His treatment of reflection in relation to the organization of perspectives is a striking instance of such insight.

\section{Post-Language Symbols}

Attention may now be directed to a kind of sign made possible by language signs and yet which is itself often not a language sign: pastlanguage symbols. These symbols may be either personal or interpersonal. We will restrict ourselves in the main to the personal variety of post-language symbols.

We have seen that there are signals and symbols which antedate language, and language signals which are not language symbols. But we can now see that from language signals and symbols a further development is possible in that these may give rise to symbols which are not themselves language signs since they do not serve as signs to other organisms when they are signs to their interpreters.

Mead has dealt with an early step in this development at some length in speaking of "the importation of the conversation of gestures into the conduct of the individual organism." 8 The "conversation of gestures" (such as the response of each dog in a dog-fight to the other dog's gestures as signs of what the other dog is about to do) is said to be

Mind, Self, and Society, p. 186. 
merely "a part of the social process which is going on. It is not something that the individual alone makes possible. What the development of language, especially the significant symbol, has rendered possible is just the taking over of this external social situation into the conduct of the individual himself." ${ }^{9}$ The individual can then "utilize the conversation of gestures that takes place to determine his own conduct. If he can so act, he can set up a rational control, and thus make possible a far more highly organized society than otherwise."10 J

What we wish to emphasize is an aspect of this process that Mead did not explicitly single out: namely, language signs, when they have appeared, may themselves be substituted for by other synonymous signs which, as substitutes, retain all the signification of the language signs and yet now function with respect to one organism alone. Thus a sound, when a language sign, can be heard by other organisms than the communicator, but certainly there is some sense in which there is still a sign-a post-language sign-when the organism does not speak aloud and so is not heard by others or by itself. This is the kind of situation which J. B. Watson referred to as subvocal talking, and which many behaviorists have identified with thinking. The point here stressed is that such a sign is not social in operation, though social in origin since it depends on language. And it is a new sign, since the original sign was a sound, while now no sound is uttered. The driver who repeats "silently to himself" the command which he first heard from another has substituted for the spoken words (an exteroceptive stimulus) some stimuli within his own body (proprioceptive stimuli). The latter are different signs and not thernselves language signs; since they are substitute signs synonymous with language signs they are properly called post-language symbols (and in this case, personal post-language symbols).

It is not hard to understand in behavioral terms how personal postlanguage signs can arise. For since a language sign, such as a sound, is produced by an action of an organism, any other stimulus within (or at times without) the organism which is produced by the action (or connected with the action) may become a substitute for the sound.

${ }^{9}$ Mind, Self, and Society, pp. 186-87.

${ }^{10}$ lbid., p. 191. 
This substitute sign is by definition a symbol; it is not a language sign in that it is not a stimulus to other organisms; it has the advantage of making available to the individual organism with the minimum of time and effort the signification of the language sign for which it is a substitute. Different organisms will in general have different postlanguage symbols as substitutes for the same language sign. These post-language symbols will then be synonymous (or highly similar in signification) but they will not be interpersonal since they will belong to different sign-families of different interpreters.

It is not necessary, though it is the most frequent case, that the personal post-language sign be a stimulus within the organism. It might be a mark on paper made by the organism (as in the case of many personal systems of notation, which differ from "writing" in that the latter is interpersonal); it might be some stimulus-object in the environment which has occurred along with language signs and so become a substitute for them (as a stone which one was fingering during a treasured conversation and which one then keeps on one's desk). Hence for each individual there are stimuli in his environment as well as in himself which have the signification of language signs and yet which are not interpersonal. In this way language continually enlarges the domain of signification within the individual's environment. The "perceived" environment has taken on a signification it could not otherwise have. The "perception" of objects as enduring and as substantial seems to serve as a striking example.

In case the stimuli in question are accessible to other organisms they may become interpersonal post-language signs, perhaps comsigns, and perhaps even elements in a language. In this way language extends its complexity to the natural and constructed objects which form the environment of a society. A development of semiotic in this direction offers great promise for a better understanding of the sense in which, and the degree to which, human culture is itself a sign phenomenon.

Mead did not work with a very clear-cut definition of language and hence did not separate as has been done here the post-language symbol from the language sign; both were included under his term 'significant symbol.' Common usage would undoubtedly support him in this, as the phrase "talking to oneself" shows. Mead himself went on to 
equate mind and thought with the operation of significant symbols: to have a mind is to utilize significant symbols in one's behavior. Our present task is not the construction of the terminology of psychology, and the semiotician need not commit himself at this point, especially since students of behavior differ on this issue. But the possibilities suggested may at least be mentioned. It may prove fruitful to regard any interpretant as an "idea" (with 'concept' possibly being limited to the interpretant of a general symbol), and to regard the difference between "talking out loud" and "thinking to oneself" as the difference between the presence of language signs and personal post-language symbols. It is then a matter of decision whether to regard every sign-process as a "mental" process or whether to limit "mind" (as Mead does) to signbehavior in which language signs or post-language symbols occur. That the distinctive characteristics of human mentality are closely related to language signs and personal post-language symbols seems certain in the light of Mead's discussion of reflective thinking. Whether the psychology of the future will, however, choose to define such terms as 'thought,' 'idea,' 'concept,' and 'mind' on the basis of semiotical distinctions must be left to psychologists.

\section{The Mentalistic Controversy Resumed}

The distinction which has been made between signal and symbol has remained within the context of behavioristics. Signals and symbols are alike signs in that they are preparatory-stimuli controlling behavior with respect to other stimuli; the symbol is a sign producible by the organism itself and a substitute for some other sign, but this difference, while distinguishing signal and symbol, is not regarded as a fundamental difference in their nature as signs. Such a view has, however, frequently been denied, and often mentalistic terms ('concept,' 'idea,' 'meaning') have been invoked to account for the difference in question. The discussion is often connected with the view that the distinction between signal and symbol answers to the difference between animals and humans, men having "symbols" in addition to signals because of their "minds." To discuss this issue is a convenient way of raising again the mentalist-behavioral controversy and at the same time of 
elucidating somewhat more fully the relation of signals and symbols, whether they be pre-language, language, or post-language signs. Susanne Langer's formulation provides a point of departure:

A sign [that is, a signal] indicates the existence-past, present, or future-of a thing, event, or condition. Wet streets are a sign that it has rained. A patter on the roof is a sign that it is raining. A fall of the barometer or a ring around the moon is a sign that it is going to rain. ... A whistle means that the train is about to start. ... A crêpe on the door means someone has just died. ${ }^{11}$

A term which is used symbolically and not signally does not evoke action appropriate to the presence of its object. If I say: "Napoleon," you do not bow to the conqueror of Europe as though I had introduced him, but merely think of him. If I mention a Mr. Smith of our common acquaintance, you may be led to tell me something about him "behind his back," which is just what you would not do in his presence. . . Symbols are not proxy for their objects, but are vehicles for the conception of objects. To conceive a thing or a situation is not the same thing as to "react toward it" overtly, or to be aware of its presence. In talking about things we have conceptions of them, not the things themselves; and it is the conceptions, not the things, that symbols directly "mean." ${ }^{2}$

Signals, on this view, "announce their objects," while symbols lead their interpreters to "conceive their objects." ${ }^{13}$ How is this difference to be explained on the behavioral approach ? $^{\mathrm{K}}$

One relevant point lies in the fact that a symbol is on the whole a less reliable sign than is a signal, since it is producible by the organism and hence may appear, when the organism has a certain need, in situations in which what is signified is not present. The signal being more closely connected with external relations in the environment is more quickly subject to corrections by the environment and hence tends to be more reliable, that is, to lose its sign status when the unreliability becomes rather great. Hence signals do on the whole "indicate existence" more certainly than do symbols. But since signals too have varying degrees of reliability, the difference remains one of degree.

\footnotetext{
"Philosophy in a New Key, pp. 57-58.

Ibid., pp. 60-61.

Ibid., p. 61 .
} 
A second difference is connected with the fact-later to be dealt with more fully-that signs become specialized in the extent to which they indicate an environment and to the extent to which they characterize the environment. Certain signs, and especially certain kinds of language symbols, attain high generality in their characterizing component and lose the specificity of indication which signals frequently have. If a person merely hears someone say 'rain,' the indication of whether it is raining now or has rained or will rain, or whether the person speaking is referring to all instances of rain, is absent in a way it is not if one hears the patter of rain-and hence the "evidential" value of the term 'rain' is relatively slight.

Connected with these two points is a third: when a sign is not reliable and not specific in indication, behavior is naturally hesitant. If the interpreter has other signs at his disposal, as in the case of beings with a language, he may under these conditions pay attention to the sign in question, attempt to formulate its signification, raise questions as to its reliability, assess the bearings of this reliability upon whatever goals he may have, and even make observations upon his own disposition to respond which is produced by the appearance of the sign. And since signs are used for many purposes other than description and prediction, signs low in reliability may still be attended to, produced, and cherished-as in the case of a work of fiction. There results in these ways the great difference between signals at one extreme and symbols at another, but again there is no reason to regard the difference as transcending a behavioral formulation.

And finally, and of greatest importance, is the fact which we have tried consistently to stress: whether a sign does or does not lead to overt behavior depends upon whether or not certain conditions of motivation and environment are fulfilled. The person who hears a patter on the roof need no more act as he would if he were out in the rain than a person who hears the word 'Napoleon' need act as he would if he were in the presence of Napoleon. Both signals and symbols involve dispositions to response and both alike may or may not occur in situations in which one acts as he is disposed to act. The difference is not between "announcing" an object in the case of signals and of "conceiving" 
an object in the case of symbols, but in the degree of absence or presence of the supporting conditions under which dispositions to behavior issue in overt behavior.

Such are at least some of the directions in which a behaviorally oriented semiotic may seek to explain in its terms the phenomena which have led others to claim that symbols (especially language symbols) and signals are so different that they should not both be called signs. It is hard to see what is added by invoking "concepts" as a principle of differentiation. Unless the term 'concept' (or 'idea' or 'meaning') is itself so defined that one can tell when concepts are or are not present, the term offers no help to semiotic. It is more promising to start with an objective approach and then to correlate if possible results so obtained with the reports of self-observation concerning the presence of concepts. It may turn out that concepts are claimed to be present when signs of a certain kind are operative (perhaps general symbols); then the question would arise whether concepts might not be identified with the interpretants of such signs (and so become a special class of "ideas" if ideas were equated with interpretants). The self-observation of concepts (and ideas) would then be the observation by an organism of certain features of its sign-behavior. But these again are matters for the psychologist to decide, and not obstacles for the semiotician, nor arguments against a terminology which embraces signals and symbols as subclasses of signs, nor principles for the explanation of the differences between signals and symbols.

\section{Signs in Animals and Men}

Once there is an agreement upon the signification of such terms as 'signal,' 'symbol,' and 'language,' the comparison of non-human and human sign-behavior is an empirical problem falling within descriptive semiotic. As such it is a specific scientific problem, and its answer is to be given by the scientific student of behavior. Without attempting such an answer here, we may notice how the problem looks in terms of the general framework which has been developed. If it is evident that sign-processes at the level of human behavior presuppose and grow out of such sign-processes as occur in animals, it is also evident that human 
behavior shows an amazingly complex refinement and proliferation of signs beyond that of any other known organism. If there is continuity in some respects, there are also impressive differences in other respects. The difficulty is in doing justice to both sets of facts.

The problem is how to distinguish animal sign-behavior from that of man. Here there are two possibilities: one is to insist that the difference is solely a matter of degree; the other is to insist that there is some unique characteristic of human signs not found elsewhere in the animal world. The first tendency is most prominent in the students of animal behavior since they are aware of the complexity of such behavior; the second is most prominent in the students of the social sciences and the humanities, since they are impressed with the unique place of signs in the subject matters with which they deal. Hence the frequent result that some student of man proposes a unique differentiation of human signs which the student of animal behavior then seeks to show is manifested in at least an embryonic form in the animal world.

There have been many proposals for a unique differentiation of human signs: men have symbols while animals have only signals; men alone have signs of signs; human beings are unique in the transmission of signs by social heredity; human beings use signs voluntarily while animals do not; animal signs occur only in perception while human beings are capable of inferences based on signs; men alone become interested in signs as goal-objects; men alone have language. The critic of such proposals denies that a sharp differentiation has been found and seeks to supply evidence for attributing the claimed characteristic to non-human animals. He need not, and usually does not, deny that these characteristics are found in an extreme degree in the signs in human behavior. That social heredity, for instance, plays a vastly more important role in the transmission of human signs than it does in animals, or that men operate at higher levels of signs than animals do, is in fact not in doubt. The question in each case is merely whether one can say: Here is a unique characteristic of human signs not shared by the signs of any other animal. And certainly most proponents of such characteristics have not convincingly made their case.

All such discussions usually culminate in the question as to whether language is unique to man. Here the issue is in part terminological, 
since if 'language' is made synonymous with 'communication' there is no doubt that animals have language; or if only some of the criteria which are incorporated in the preceding definition of language are insisted upon (or different criteria), then too there may be no doubt about the existence of animal language. But if the full proposed definition is accepted, I know of no convincing evidence that any animals other than men have either a signal or a symbol language, though it is to be insisted that the problem is an empirical one and not to be dogmatically resolved. That animal signs at times occur in social acts, that animal signs are often interpersonal and plurisituational, that an animal may itself occasionally produce the sign which it interprets, that some symbols occur in animal behavior does not seem to be in doubt. And yet these facts are not themselves sufficient conditions for attributing language to animals.

The fact that a sign is plurisituational and interpersonal does not make it a language sign, nor does the mere addition of the fact that the sign is produced by an organism. It is further essential that the sign have the same signification to the producers of the sign when produced by them that it has when produced by other organisms, that is, that it be a comsign; and it is by no means clear that this condition is fulfilled in sub-human sign-behavior. Hence even if only the first four of our criteria for a language sign were accepted as a definition, it still remains to be shown that these conditions are all jointly met in living beings other than men. But even if these conditions were met, the fifth requirement is a harder hurdle. For though animal signs may be interconnected, and interconnected in such a way that animals may be said to infer, there is no evidence that these signs are combined by animals which produce them according to limitations of combination necessary for the signs to form a language system. Such considerations strongly favor the hypothesis that language-as here defined-is unique to man. ${ }^{\mathbf{L}}$

Even if empirical evidence, possibly gained by novel experimentation, should modify this hypothesis, it is not likely to refute the view that man is characteristically the linguistic animal. And certainly no other animal transmits to such degree its signs by social heredity, attains signs so highly independent of particular situations, employs symbols to such 
an overwhelming extent, uses signs (voluntarily and involuntarily) for so many purposes, is so attentive to its signs as signs, passes to such high levels of sign-behavior, performs such complex inferences via signs, or so often makes of signs themselves goal-objects. In all these ways, human language (and the post-language symbols it makes possible) goes vastly beyond the sign-behavior of animals. But languagebehavior is still sign-behavior, and language signs rest upon, and never, completely take the place of, the simpler signs which they presuppose. The continuity is as real as the discontinuity, and the similarity of human and animal signs-behavior as genuine as the difference.

The present approach thus sharpens, though it does not itself solve, the empirical problem as to the relation of sub-human and human signbehavior. By refining the terminological apparatus of semiotic and formulating such terms behaviorally, it leads to a more precise statement of the general problem, namely: which kind of signs (signals, symbols, comsignals, comsymbols, language signals, language symbols, and post-language symbols) appear in which organisms' behavior, and what are the evolutionary and genetic relations between the signs which do appear ? ${ }^{\mathbf{M}}$ It is easy (and perhaps correct) to say that signals rather than symbols predominate in animal behavior and that language signs (and post-language symbols) are mainly, and perhaps exclusively, human attainments. But there are a vast number of sign-processes which bridge the gap between these extremes and which presumably serve to connect in evolutionary terms sub-human and human signbehavior. It is these intermediary sign-processes (symbols, comsigns, etc.) to which attention is here called and about which further knowledge is needed. Some of this knowledge can be gained from existing literature; much of it must be the product of specially designed experimentation. As it is obtained the account of biological evolution will be greatly and significantly enriched.

\section{Language and Behavioristics}

Behavioristics naturally attempts to supplement its description of behavior in molar or macroscopic terms by isolating the detailed mechanisms operative in such behavior. This has been, for instance, the 
direction of development in the work of Mead, Tolman, and Hull. So far we have given a rather gross description of sign-behavior, attempting to distinguish it from other behavior; the next step would be to explain in detail the operation of sign-behavior. Since the foundations of behavioristics are themselves matters of controversy today, semiotic must here move cautiously, leaving the details to properly equipped scientists. Nevertheless, while not committing semiotic to any existing system of behavioristics, it may be useful to show that there already exists the possibility of relating sign-behavior to the more general categories of behavior theory. Clark L. Hull's terminology will be chosen for this illustrative purpose.

That Hull should have no insuperable difficulty in accounting for learned sign-behavior in his terms is easily seen. ${ }^{\mathrm{N}}$ For the notion of a behavior-family, it will be remembered, is a special case of his notion of habit-family, abstracted from certain further assumptions of Hull as to the relation between the response-sequences constituting a family (such as the assumption that the strengthening of one responsesequence strengthens the tendency to perform other response-sequences of the family in question). The other basic term which has been used - 'preparatory stimulus'-might be treated, as Hull has suggested, in terms of what he calls temporal stimulus patterning. Temporal stimulus patterning in his account is distinguished from simultaneous stimulus patterning in that "the onset of the several stimulus energies occurs successively." 14 In his terms the basic principle of learning, crudely stated, is as follows: If a stimulus occurs at about the same time as a response which reduces a need, the stimulus tends in the future, when the need is operative, to produce the response in question. ${ }^{\circ}$ If now a temporal compound is the stimulus in question (that is, a stimulus compound) it may well be the case that no response will be made to the first stimulus (that is, the preparatory-stimulus) though the occurrence of this stimulus is a necessary condition for the response in question to occur when the later members of the stimulus pattern appear. There should then be no objection on Hull's approach to calling such a preparatory-stimulus a sign in case the response in question is

\footnotetext{
${ }^{14}$ Principles of Behavior, p. 350.
} 
a response-sequence of a behavior-family-though of course Hull may not himself care to use the sign terminology. ${ }^{P}$ Such a sign is even an equivalent stimulus ${ }^{15}$ for the stimulus which originally called out the response, provided 'stimulus equivalence' be defined not solely in terms of the identity of response called out by two stimuli but is extended to stimuli which call out response-sequences of the same behavior-family. Hull attempts to explain temporal patterning in terms of the connection formed between the trace left by the earlier stimulus and the later stimulus. $^{16}$ Insofar as this can be done, and the notion of behaviorfamily derived from his general principles, Hull's system would be able to incorporate sign-behavior. The symbol would present no especial problem, since his notion of the pure-stimulus act provides the stimulus-producing response needed for a symbol, while the explanation of higher order conditioning in terms of the principle of secondary reinforcement ${ }^{17}$ shows how one sign may be substituted for another-and indeed shows what is meant by such substitution.

It is also true that none of the five criteria proposed for the language sign fall outside the scope of Hull's conceptual scheme. That the sign be producible by the organism is a special case of the production of purestimulus acts. The special interpersonality of the language sign (that is, the fact that it signifies to its producer what it signifies to other organisms) is presumably dependent on the principle of stimulus generalization: "The reaction involved in the original conditioning becomes connected with a considerable zone of stimuli other than, but adjacent to, the stimulus . . . involved in the original conditioning." 1 s Hence because of stimulus generalization a sound produced by an organism will tend to have the same effect on itself as a similar stimulus produced by another organism. The special plurisituational character of the symbol (its relative independence of a particular external environment) is consonant with Hull's principle that the stimuli produced by a need may become conditioned to reactions just as do other stimuli. ${ }^{19}$

\footnotetext{
${ }^{15}$ See "The Problem of Stimulus Equivalence in Behavior Theory," Psychological Review, 46, 1939, pp. 9-30.

${ }^{18}$ Principles of Behavior, pp. 370-71.

${ }^{17}$ Ibid., pp. $85 \mathrm{ff}$.

${ }^{18}$ Ibid., p. 183. Chapter 12 is entitled "Stimulus Generalization."

${ }^{18}$ lbid., p. 240.
} 
Because of this fact the organism may engage in elaborate symbolic processes when the environment which originally produced the signals for which the symbols are substitutes no longer provides the signals formerly provided. In some such way as this Hull's version of behavioristics could presumably account for all the phenomena involved in comsigns, and thus handle the first four of the criteria requisite for language signs.

The fifth criterion requires that language signs be combinable with each other in certain ways and not in others, and that these combinations form a complex sign whose constituents are themselves signs. Hull has suggested that such combinations are to be explained in terms of simultaneous and temporal stimulus patterning, since "each of the forty or so elementary speech sounds is a fairly distinctive pattern. . . . Each of the thousands of words of the better-developed languages consists of a temporally patterned sequence of these elementary speech sounds, stops, and so forth. In reading, each letter is a complex visual pattern, each word is a complex pattern of these letter patterns, and each sentence is a temporally patterned sequence of printed word patterns." ${ }^{20}$ Sign-families can be compound signs as well as uncompounded signs; the problem of the genesis of a grammar is the problem of accounting for the selection of some combinations of signs as signfamilies and not others. Since the acquisition of stimulus patterns presumably follows the same laws of learning as do non-patterned stimuli, the acquisition of the grammatical possibilities and restrictions for the use of signs is in principle statable in Hull's terms. ${ }^{\mathbf{e}}$ It is by satisfying certain needs when and only when signs are combined in certain ways that the child learns to combine signs in some ways and not in others. And since a pattern of stimuli can become a compound stimulus without the component stimuli losing their stimulus character, some insight is gained as to how a combination of signs may have its own distinctive signification while the factors in the combination continue to retain their sign status. In some such way the grammatical features of language may be approached from Hull's point of view. Post-language symbols add no special difficulty, since it has already been explained

\footnotetext{
${ }^{20}$ Principles of Behavior, p. 397 . Chapter 19 is entitled "The Patterning of Stimulus Compounds."
} 
how other stimuli produced when a sound is produced may by conditioning become substitutes for the sound; Hull's concept of individual pure-stimulus act is here applicable.

We repeat that the preceding remarks are at best suggestive; they do not intend to commit semiotic to Hull's theory of behavior nor even to deal adequately with sign-behavior in terms of Hull's theory. But they may serve to illustrate how sign-behavior, as formulated in the present account, lends itself to treatment within the categories of a general theory of behavior. Vague speculation on these matters is no longer necessary; the problems of sign-behavior have already reached the stage of empirical formulation and possible experimental resolution. And let me add that until any particular theory of behavior explains in its own terms the phenomena of sign-behavior which we have isolated, it will be an inadequate instrument for dealing with the more complex and distinctive features of man and his culture. 


\section{MODES OF SIGNIFYING}

\section{The Problem Stated}

7 HE discussion up to this point has aimed to lay the foundations for semiotic within behavioristics. The intention has been to show how the basic terms of a science of signs can be formulated in terms descriptive of behavioral processes. The question now arises as to what light this approach can throw upon the issues with which inquirers in this field are occupied. The first of these is the central problem of the differentiation of the major modes of signifying.

This problem is in the forefront of contemporary semiotic. It appears in the current distinction between "referential" and "emotive" terms, a basic distinction in the work of C. K. Ogden and I. A. Richards. ${ }^{\Delta}$ The contrast occurs in many other forms: various writers distinguish cognitive and non-cognitive signs, cognitive and instrumental signs, referential and expressive signs, referential and evocative signs, and then refine these basic dichotomies by various and varying subdivisions of each of the contrasted classes. The almost universal utilization of such distinctions is evidence that fundamental issues are involved, while the wide range of differences of opinion in these matters suggests that analysis has not isolated clearly the factors which the various distinctions aim to formulate. The task of the present chapter is to approach this field behaviorally. We shall try to show how in behavioral terms the distinctions between such sentences as 'There is a deer,' 'What a fine fellow!', 'Keep the wind ahead!' and such terms as 'there,' 'deer,' 'fine,' 'keep' can be formulated. Corresponding to 
such sentences and terms a distinction will be made between designative, appraisive, and prescriptive ascriptors, and between identifiors, designators, appraisors, and prescriptors as signs in various modes of signifying. To these a fourth kind of ascriptor and a fifth mode of signifying will be added: formative ascriptor and formators as signs signifying in the formative mode. These terms are intended to include such a sentence as 'It will rain or not rain,' and the signs 'or' and 'not' in this sentence.

Before entering upon the task of formulating the modes of signifying, the grounds of possible confusion must be noted. Signs may be classified in terms of their significata (kinds of significata, relations between their significata), in terms of their denotata (whether a sign denotes, is reliable, is iconic), in terms of how they are combined with other signs (the position of the sign in a sentence, the form of the sentence), or in terms of their relation to their interpreters (whether produced by the interpreter, believed, provocative of emotion, useful to the interpreter). A comprehensive classification of signs would take into account all of these possibilities; almost every page of this study bears upon the topic in one way or another. The classification of the major modes of signifying is not itself an exhaustive classification of signs, but only a fragment of such classification.

Many of the current differences in the attempts to differentiate "statements," "valuations," and "imperatives" comes from the different bases upon which the various classifications are made. The central issue is whether these modes of signifying are to be explained in terms of differences in the signification of the signs or in terms of different uses and effects of the signs or in terms of both sets of criteria. There is a common tendency to say that such a term as "war' is "referential" if it produces no emotion in its interpreter and "emotive" if it does produce an emotion; a term such as 'good' is at times claimed to be merely an "emotive" (or "expressive") term and to lack "referential" signification. The extensive controversy over such attempts to differentiate the modes of signifying indicates that there are various possible bases for making the intended distinctions.

It is of course true that signs may be classified, among other ways, in terms of the emotions they produce or the states of the producer 
they express or the purposes which they serve or thwart. But this is no substitute for (nor a rival to) a classification in terms of kinds of significata. The important thing is not to confuse the different possible bases of classification nor to substitute one for the other. It may be the case that an interpreter of the term 'good' is in general more emotionally aroused than an interpreter of the term 'deer,' and if so there is no harm in calling 'good' an emotive term; it does not necessarily follow however that there is no difference in the kind of signification of these terms or that 'good' signifies an emotion.

These confusions are facilitated by the failure of behavior theory to give criteria for the differentiation of modes of signifying in terms of differences in interpretants and hence in terms of what is signified. The following pages will attempt to show that our previous formulation permits of this differentiation in terms of differences in tendencies to response, and that it helps to avoid the confusion involved in the appeals to emotion, expression, and use as a basis for this differentiation. Succeeding chapters will then be concerned with the uses and effects of signs in various modes of signifying.

\section{Origin of the Modes of Signifying}

There are three major factors discriminable in sign-behavior corresponding to the nature of the environment in which the organism operates, the import or relevance of this environment for the needs of the organism, and the ways in which the organism must act upon the environment in order to satisfy its needs. These three factors, when signified, may be called respectively the designative, the appraisive, and the prescriptive components of signifying. Thus the words of the speaker may designate a certain condition of the road, appraise this condition as an obstacle to further progress, and prescribe the discontinuance of the response of driving to the point in question; the buzzer perhaps designates food in a certain place, appraises this positively in relation to hunger, and prescribes the response of acting in a certain way. To say that sign-behavior has these aspects is to make an objective statement about the behavior of an organism in a situation: namely, that the organism because of the sign is prepared 
to react to certain features of its environment, to respond preferentially to some of these features in virtue of a certain need, and to favor some response-sequences rather than others in the attempt to satisfy this need. What is designated, how it is appraised, and what responses are prescribed are to be determined by an analysis of the sign-behavior of the organism in relation to an environment.

All sign-behavior involves these components in varying degrees; the differences in the modes of signifying in question may be stated in terms of the degree to which the various components are present in various signs. The genetically earlier signs in animal and human behavior lack such differentiation. The origin of these differences may be explained by the relative independence of environment, need, and response. Water may be present in the environment both when the organism is thirsty and when it is not thirsty; different ways of acting under different circumstances are required to attain water to drink; and many of the responses useful in obtaining water may also serve to attain or frustrate various needs with respect to things other than water. The total signification of a sound which is a sign in one situation may thus be very different from the total signification of a similar sound in a different situation; to this extent the sign is not plurisituational. And yet certain of the components of the signification may be similar in the various cases. Both a hungry and a satiated animal, for instance, may be disposed to respond by food-seeking behavior when a buzzer sounds, but given their different needs and spatial positions, the object designated has a different import for the two animals and calls for different responses; here the designative component is similar in the two cases but the appraisive and prescriptive components widely different. And analogous cases are easily obtainable where the variation lies in the other components. Poets who differ widely in what the term 'god' designates may agree in appraising god as an object of supreme value; a command such as "Come here!" may signify with high constancy the requiredness of the response which it prescribes and yet different interpreters may differ widely in their appraisals of what the signified response will lead to and in their reactions to the command.

Because of this relative independence of environment, need, and 
response certain signs become specialized in signifying predominantly one or another aspects of the various situations in which they appear. The differentiation of modes of signifying is thus connected with the extent to which a sign is general and plurisituational; hence it is with symbols, and especially language symbols, that the differentiation is most prominent. Deer may stand in a variety of relations to various needs, and the actions appropriate to deer differ with these needs; consequently a sign such as 'deer' may become so general with respect to its signification of the importance of deer, and how they are to be reacted to, that it practically loses its appraisive and prescriptive elements and becomes primarily a sign in the designative mode of signifying. To call a deer "fine," however, is to signify that the object designated has a positive import for behavior, so that 'fine' signifies primarily in the appraisive mode of signifying; what it designates and what action it prescribes are correspondingly vague and differ from situation to situation. Similarly, to say that deer should be approached against the wind signifies a special response-sequence to be performed with respect to a goal. 'Should' itself is thus a very general sign signifying some action or other as prescribed, and is a prescriptor; what object is designated and how it is appraised recede into the background. Each of these specialized modes of signifying needs the differentiation of the other modes as it itself becomes differentiated: as signs become primarily designative and neutral with respect to the signifying of the import of an object and what is to be done about it, other special devices are needed to care for these neglected aspects, and appraisors and prescriptors assume this task.

\section{Behavioral Criteria for Differentiating the Modes}

Signs in the different modes of signifying signify differently, have different significata. We now consider the problem of how these differences may be formulated behaviorally.

The general direction of analysis is dictated by our previous treatment of what is meant by the significatum of a sign; the modes of signifying will correspond to the major kinds of significata. A significatum, however, as the condition under which something is denoted by the 
sign, always involves an interpretant (a disposition to respond in certain ways because of a preparatory-stimulus); hence the major kinds of significata must be distinguished in terms of distinctions between interpretants, that is, in terms of differences in dispositions to respond. What then are the main kinds of dispositions to response which can occur in sign-behavior?

An exact formulation of the differences in interpretants is a difficult matter. The task will be facilitated if we keep to a concrete example, for this provides us with denotata of the signs which we wish to introduce in semiotic; the formulation of what is involved in such denotation would give the significata of the signs which we seek.

Let us consider a dog whose hunger and thirst we can control, and for whom signs have been established which signify meat or water in various places. Let us suppose however that the sign-behavior of the animal can be complicated in the following ways. Certain stimuli $\left(I_{1}, I_{2}\right)$ are such that when $I_{1}$ is presented to the dog it seeks food or water as the case may be at place 1 , and when $I_{2}$ is presented, it does so at place 2. $D_{1}$ and $D_{2}$ are such that if the dog is hungry it responds by food-seeking behavior when $D_{1}$ is presented, and when thirsty by waterseeking behavior when $D_{2}$ is presented. Let $A$ be a stimulus such that when it is combined with other stimuli the dog acts as follows: If it is hungry and is presented in close succession with the combined stimuli $I_{1} D_{1}$ and $I_{2} D_{1}$, only one of which combinations is accompanied by $A$ (that is, $A I_{1} D_{1}$ or $A I_{2} D_{1}$ ), it always seeks food at the place corresponding to the $I$ in the combination in question (that is, at place 1 when it is presented with $A I_{1} D_{1}$ followed or preceded by $I_{2} D_{1}$, and at place 2 when presented with $A I_{2} D_{1}$ followed or preceded by $I_{1} D_{1}$ ); a similar situation is found when it is thirsty and $D_{2}$ replaces $D_{1}$. Suppose further that there is another stimulus $P$ such that when and only when $P$ is introduced in combination with other stimuli (such as $P I_{2} D_{\mathbf{1}}$, $\left.P A I_{1} D_{2}\right)$ the response-sequence of the dog includes some such specific response as turning in a circle three times when it goes for food or water.

Now under these circumstances the various stimuli $I, D, A$, and $P$ have specific influences upon the behavior of the dog, and so upon its dispositions to respond. All of them are preparatory-stimuli influ- 
encing the responses which the animal is disposed to make, but they influence these responses in quite different ways. The l's dispose the dog to orient its response-sequences to a particular spatio-temporal region; the $D^{\prime} s$ dispose the dog to response-sequences of a particular behavior-family (behavior to food or to water as the case may be); $A$ disposes the dog to respond preferentially to food at one place rather than another, or water at one place rather than another; $P$ disposes the dog to perform certain response-sequences rather than others (that is, those which include the responses of circling three times).

Let us now further suppose that $D_{1}$ and $D_{2}$ do not by themselves dispose the dog to seek food or water in one place rather than another; $I_{1} D_{1}$ when presented with $I_{2} D_{1}$ does not cause the dog to go to one place rather than another; $A$ and $D_{1}$ do not dispose the dog to turn in a circle; and so on for the other cases. Then to the degree that this is true, $I, D$, $A$, and $P$ are signs with distinguishable kinds of interpretants, and so with distinguishable kinds of significata. Signs of the kind represented by $I, D, A$, and $P$ are then respectively identifiors, designators, appraisors, and prescriptors. Formators will be left for later treatment; it will be maintained that such signs presuppose signs in the other modes of signifying and arise from the need of distinguishing how signs signify in specific sign-combinations. The formulation of the kinds of interpretants involved in these various signs gives us the criteria for distinguishing the major modes of signifying.

In the case of identifiors the interpreter is disposed to direct his responses to a certain spatial-temporal region; in the case of designators the interpreter is disposed toward response-sequences which would be terminated by an object with certain characteristics; in the case of appraisors the interpreter is disposed to respond preferentially with respect to certain objects; in the case of prescriptors the interpreter is disposed to perform certain response-sequences rather than others. So identifiors may be said to signify location in space and time, designators to signify characteristics of the environment, appraisors to signify preferential status, and prescriptors to signify the requiredness of specific responses.

It is perhaps desirable to have special terms for the special kinds of significata involved in signs in the various modes of signifying. We 
will use locatum, discriminatum, valuatum, and obligatum as signs signifying the significata of identifiors, designators, appraisors, and prescriptors. The reader who fears that we are in this way peopling the world with questionable "entities" need only be reminded that these terms refer only to the properties something must have to be denoted by a sign, that is, to permit the actualization of the response-sequences to which the interpreter of the sign is disposed. So while the terminology is clumsy, and need not often be used, it is harmless enough and still behavioral in formulation.

\section{Expression, Emotion, and Usage}

Identifiors, designators, appraisors, and prescriptors are all signs in the sense in which that term has previously been employed. They prepare in various ways the behavior of their interpreter with respect to that for which they are preparatory-stimuli: the identifior determines the region upon which behavior converges; the desginator tends to restrict the response-sequences which are prepared to objects of a certain kind; the appraisor directs responses in terms of the import or relevance of certain objects; the prescriptor specializes behavior even further by restricting the specific response-sequences which the interpreter is prepared to make.

We are now in a position to compare and relate this analysis of the modes of signifying to those accounts which are based upon an appeal to expression, emotion, and the use of signs to accomplish certain purposes. Such analyses gain their support in the fact that there is often a close correlation between the production of a certain sign and certain states of the producer of the sign. This correlation may exist with respect to the way the sign is produced as well as with respect to the signification of the sign produced. An excited person may speak with greater intensity, shorter utterances, more rapid speech than a person who is not excited, but equally well he may differ as to what designators he utters and the number and kind of appraisors or prescriptors which he employs. Hence the manner of production of signs and the kinds of signs produced may themselves be to the producer of the sign or to other persons signals of the state of the producer of the 
sign. This is a common situation, and such signs can be called expressive signs. A sign on this usage is expressive if the fact of its production is itself a sign to its interpreter of something about the producer of the sign. ${ }^{\mathbf{B}}$

But the point that needs to be stressed is that such "expressiveness" has nothing to do with the mode of signifying of signs, since any sign whatsoever may be expressive: a consistent use of designators is as "expressive" of their producer as is a consistent use of appraisors or prescriptors. The expressiveness is not part of the signification of the sign in question but rather the signification of another sign, namely of a sign which consists in the fact that a certain sign is produced. That a person often talks in the language of physics may itself be a sign of his interest in certain things rather than others, but this does not make the terms of physics include as their significata states of the users of the terms. Similarly, the fact that appraisors are normally used by persons with certain needs makes it often possible to infer from their use the existence of a given need, without the appraisor itself signifying this need. The expressiveness of signs is thus an additional property of signs over and beyond their signification, and not a basis for the differentiation of the modes of signifying.

The same considerations apply to emotions. Emotions may be signified, and emotions may be correlated in various ways with signs as produced or as interpreted. But the signs which signify emotions need not be appraisors, and appraisors may but need not signify emotions. 'Emotion' is not an emotional term; and one can correctly interpret the signification of a poem praising dogs without oneself liking dogs, without feeling emotional when reading the poem, or without even regarding the poem as signifying that its author liked dogs.

An analogous situation obtains with respect to the various purposes with respect to which signs may be employed. Prescriptors, for example, are normally used by persons who wish to secure the performance of the actions signified as required, and so the receiver of a command will normally take the fact that the command is made as a sign of the wishes of the producer. But even here this may not be done, as in the case where the producer of the sign is known to be in the hands of the enemy; the interpreter might then recognize that the 
sign is a prescriptor and yet not regard it as signifying the wish of the utterer that the action signified be performed. Signs may be employed to realize many purposes, and their employment may be closely correlated with certain purposes of their producers, but such a correlation gives in itself no basis for the differentiation of the modes of signifying.

It does not follow from this discussion that the terms 'expressive sign' and 'emotive sign' and 'instrumental sign' are in themselves objectionable. The point, rather, is to distinguish the features which enter the signification of a sign from the additional information which may be gained about someone from the fact that he produces a certain sign. Much of the contemporary discussion of the differences between kinds of signs, and between the sciences and the humanities, fails to make this distinction. For certain purposes the mixing of these matters is without much importance, but for the underlying issues in the development of semiotic, as well as for the understanding of the arts, religion, the sciences, morality, and politics, confusion at this point is disastrous.

Having made the distinctions in question, and shown how the modes of signifying can be isolated behaviorally in terms of what is predominantly signified by a sign to its interpreter, it is fair to admit the frequent correlation between these kinds of signs and the material which the doctrines of expression, emotions, and usage have as their basis. Designators, for instance, are frequently to self-observation "cold" terms, appraisors "warm" terms, prescriptors terms accompanied by a "sense of obligation." Such feelings and reports about them may therefore be used with caution as indirect evidence that a sign is in a certain mode of signifying. But since the reliability of this evidence is not always high and can only be assessed if one can isolate the kinds of sign by some other criterion, these feelings and reports are not the keystones of a scientific semiotic.

\section{Ogden and Richards on Modes of Signifying}

Since the work of C. K. Ogden and I. A. Richards has been especially influential in the contemporary world of semiotic in the discussion of the modes of signifying, a brief account of their distinctions will further 
serve to underline the approach which is here being followed. Their volume, The Meaning of Meaning, was a pioneer attempt to distinguish the signification of signs represented in its purest form in science from the kinds of signification characteristic of non-scientific discourse; the present chapter has a similar intent, but seeks to find behavioral criteria for the distinction, proceeding in the spirit of the statement in The Meaning of Meaning that "the ultimate settlement of the matter can hardly be expected until tests in some way independent of the opinion of the speaker are obtained." 1 We have attempted to supply these tests lacking in the analysis given in The Meaning of Meaning itself.

Ogden and Richards make basic the distinction of referential (or symbolic) and emotive terms. This distinction might seem to correspond in general with the present distinction of identifiors and designators on the one hand and appraisors and prescriptors on the other. But instead of making the distinction solely in terms of signification, their analysis introduces both the notions of expressiveness and use or function. So emotive terms are described as "the use of words to express or excite feelings and attitudes" ${ }^{2}$ and are linked with certain of the main functions of language as a means of communication. ${ }^{3}$ Four main functions are distinguished: "symbolization of reference," "expression of attitude to listener," "expression of attitude to referent," "promotion of effects intended"; in a number of places these are distinguished by the terms 'sense, 'feeling, 'tone,' and 'intention.' Referential or symbolic terms are terms which are used to perform the first function, emotive terms are those used to perform the remaining functions. Appraisors would then correspond to terms which are used to express an attitude, and prescriptors to terms used to promote an intended effect. The question then arises as to the criteria for determining when a sign is expressive and when it is used intentionally to achieve some result.

It is significant that the key word 'express' is not itself explicitly defined in the volume. But the following passage perhaps indicates what is involved in their account:

\footnotetext{
${ }^{1}$ The Meaning of Meaning, 5th edition, pp. 125, 126.

Ibid., p. 149.

Ibid, pp. 226-27.
} 
Besides symbolizing a reference, our words are also signs of emotions, attitudes, moods, the temper, interest or set of the mind in which the references occur. They are signs in this fashion because they are grouped with these attitudes and interests in certain looser and tighter contexts. Thus in speaking a sentence we are giving rise to, as in hearing it we are confronted by, at least two sign-situations. One is interpreted from symbols to reference and so to referent; the other is interpreted from verbal signs to the attitude, mood, interest, purpose, desire, and so forth of the speaker, and thence to the situation, circumstances and conditions in which the utterance is made. The first of these is a symbol situation as this has been described above, the second is merely a verbal sign-situation like the sign-situations involved in all ordinary perception, weather prediction, etc. Confusion between the two must be avoided, though they are often hard to distinguish. Thus we may interpret from a symbol to a reference and then take this reference as a sign of an attitude in the speaker, either the same or not the same as that to which we should interpret directly from his utterance as a verbal sign. ${ }^{4}$

A sign, according to this account, is "emotive" if someone takes the fact of its production as itself a sign of some state of the producer which often accompanies the production of the sign. This as we have seen may and often does happen; we have defined the expressiveness of a sign in precisely these terms. But since any sign may be so considered, and since a sign based on taking the production of a sign as itself a sign of the producer is no less "referential" than any other sign, no distinction of emotive from referential meaning really issues from the analysis.

At times another criterion is proposed: "The best test of whether our use of words is essentially symbolic or emotive is the question-'Is this true or false in the ordinary strict scientific sense?' If this question is relevant then the use is symbolic, if it is clearly irrelevant then we have an emotive utterance." 5 But the authors themselves immediately point out that the term 'truth' has both referential and emotive usages, and this fact, together with the frequent controversy and disagreement as to the sense in which, say, poetry is "true," makes the truth criterion a difficult one to employ in practice.

Add to this the fact that a poem may be written to realize any one

1 The Meaning of Meaning, 5th edition, pp. 223-224.

Ibid., p. 150. 
of a number of purposes, and our contention is strengthened that an account of the differences in signification between identifiors, designators, appraisors, and prescriptors is not to be given in terms of the expressiveness of signs or the uses to which signs may be put. Every sign has psychological and physiological accompaniments; every sign which is produced may be taken as a sign of its producer; every sign may be used for the accomplishment of a variety of purposes. The differences in the modes of signifying must be sought elsewhere, namely in the differences in what is signified, such differences themselves being explained in terms of the objects to which the sign directs behavior, their preferential status in relation to the goals of the organism, and the ways in which the organism is prepared to behave to those objects. In this manner we keep the distinctions which Ogden and Richards wish to make between referential and emotive modes of signifying and yet anchor these distinctions in objectively determinable criteria. In so doing we but move further in the direction which they themselves have seen to be desirable.

\section{Ascriptors}

Identifiors, designators, appraisors, and prescriptors influence the behavior of their interpreters in different ways, determining the location upon which it is directed, preparing it for objects with observable properties which permit the completion of responses in specific behavior-families, causing it to give preferential attention to certain objects rather than others in terms of their relevance to organic needs, strengthening the tendency to respond by some response-sequences rather than others. Since they answer the questions of where found? what characteristics? why relevant? how respond? they are respectively where, what, why, and how signs. Since behavior needs guidance on all these points, signs in the various modes of signifying complement each other: the identifiors normally serving to locate objects signified by other signs, the designators normally characterizing objects or situations otherwise identified by other signs, the appraisors normally signifying the relevance of what is otherwise identified and 
designated, the prescriptors normally signifying the responses required by something which is identified, designated, and appraised.

Yet signs in all modes of signifying do not occur in all sign-behavior. In the case of language communication the situation may supply many of the clues which behavior requires, and the communicator supplies only the additional clues which are necessary to the communicatee, such as identifying an object by signs but not signifying it further, or prescribing a response without designating the kind of object to which the response is to be made. In case the communication is by writing the situational clues are absent, and must be supplied by additional signs. In a situation in which thirsty people are seeking for water, 'Water!' may be sufficient; but 'Water!' printed in isolation on a page leaves unanswered the questions of where and why and how. Behavior needs direction with respect to what is relevant to its goals, what will be encountered as behavior proceeds, where it will be encountered, and what to do about it. And signs in various modes of signifying are combined to meet these requirements.

At the minimum signs useful to an organism must orient its behavior in space and time and prepare in some way its behavior to the region of its environment which is identified. This makes signs which bring together the identificative mode of signifying with a sign (or signs) in some other mode (or modes) of signifying of basic importance. We will call such a sign complex (and a combination of such sign complexes) an ascriptor. ${ }^{\circ}$ An ascriptor is undifferentiated if the same sign-vehicle is in various modes of signifying; the buzzer in the case of the $\operatorname{dog}$ is an example, since it identifies a location and also signifies something more about what is identified. An ascriptor is differentiated to the degree that distinct sign-vehicles carry the various modes of signifying involved. One may point to an object and say 'black' or one may say 'the object at which I am looking is black.' Where all the signs are lansigns, the ascriptor is a lanascriptor. The notion of ascriptor corresponds roughly to the term 'sentence,' though this term is usually limited to differentiated lanascriptors and to dominant (as opposed to subordinated) ascriptors. The earliest sign-processes are undifferentiated ascriptors. The isolation of the 
signs which make up differentiated ascriptors is usually done by the comparison of ascriptors. In both these senses "sentences" are genetically prior to "words"; hence to call an ascriptor a sign complex does not mean that there were signs before ascriptors. The common languages carry the differentiation of the modes of signifying very far, and much of the work of the linguist lies in describing the specializations of signs with respect to modes of signifying (as in the discussions of parts of speech) and the various forms which ascriptors take in various languages (as in the classification of kinds of sentences).

Ascriptors may be distinguished in terms of the modes of signifying of their component signs. An ascriptor composed of an identifior (or identifiors) and a designator (or designators) will be called a designative ascriptor; in a similar way we distinguish appraisive ascriptors, prescriptive ascriptors, and formative ascriptors. 'That is a deer,' 'He is a fine fellow!,' 'Keep the wind ahead!', 'He will see us or he won't' are illustrations. Since an ascriptor may involve signs in all the modes of signifying, it is necessary to have some means of distinguishing the various cases. Accordingly we propose to classify ascriptors in terms of the mode of signifying which is dominant. How is this to be determined?

An ascriptor identifies something (or a number of somethings) and signifies something more about what is identified. But this more may itself be complex. 'That is a fine deer' (spoken in an actual situation) identifies an object, designates it as a deer, appraises it as fine. Shall we say that the ascriptor is dominantly designative or appraisive? Obviously there are two ascriptors involved, one designative and one appraisive ('That is a deer,' 'That is fine'). On what basis can one say that the original ascriptor is dominantly designative or appraisive? Languages have various devices to show the subordination of the signs within a compound ascriptor, as is instanced in the contrast between 'That is a fine deer' and 'That deer is fine' (or between ' $x+(y \times z)=k$ ' and ' $(x+y) \times z=k$ '). The behavioral correlate is the ordering of the tendencies to respond involved in a number of interpretants. The sign whose interpretant is not subordinated to other interpretants will be called, after Manuel Andrade, the dominant sign of the ascrip- 
tor. An ascriptor may then be classified in terms of whether its dominant sign is a designator, an appraisor, a prescriptor, or a formator.

Languages normally supply various clues for determining what is the dominant sign. Where no such device exists in the case of an ascriptor composed of other ascriptors, the ascriptor is simply a compound ascriptor. In this case it is best to describe the compound ascriptor in terms of the relative proportion of its component ascriptors: "predominantly designative," "equally appraisive and designative," "60\% designative and $40 \%$ appraisive," as the case may be. "That is a fine deer' is then designative or appraisive depending upon whether the form involves a subordination of the ascriptors it contains; if it does not then it is equally appraisive and designative by our criterion. Here as elsewhere we must be careful to distinguish whether we are talking about a specific ascriptor sign-vehicle or are making a statistical statement about an ascriptor sign-family.

What are frequently called the "subject" and the "predicate" of an ascriptor seem to coincide with the distinction between subordinate and dominant signs, and not, as might at first be supposed, with the distinction between identifiors and signs in the other modes of signifying. In a terminology later to be employed, the distinction of subject and predicate is a syntactical distinction, while the distinction between the modes of signifying is semantical. The "subject" of "That deer is fine' is the signs 'that deer' and the predicate is 'fine' ('is' we leave for the later discussion of formators). "Subject" and "predicate" is a distinction resting on the subordination of signs, while identifiors are signs in a special mode of signifying. There is one subject in a sentence, but there may be many identifiors. In ' $A$ hits $B$, ' $A$ ' and ' $B$ ' together form the subject and 'hits' the predicate (or dominant sign). The reader will have noticed that the term 'identificative ascriptor' has not appeared. This term seems unnecessary since it is doubtful if any combination of identifiors alone would make an ascriptor. Examples which appear to be exceptions (' $A$ is here') seem to involve designators, and hence to be designative ascriptors. In any case we will not deal specifically with identificative ascriptors; if such ascriptors occur their analysis must be left to other writers. 


\section{Identifiors, Designators, and Designative Ascriptors}

Identifiors, as signifying locations in space and time (locata), direct behavior toward a certain region of the environment. But since behavior is never to a spatial-temporal region as such, but to various objects, identifiors occur in a context in which objects of a certain kind are involved -objects which are already being sought for or objects which are signified by other signs. If a child is searching for its doll, we may merely point in a certain direction to give it the clue it needs, or we may point in a certain direction and say 'doll' even when the child is not concerned with the doll. An identifior, then, signifies the location of something or other, but does not itself signify anything else about this something or other. It determines where and when behavior will be directed toward something; the kind of behavior must be otherwise determined. The identifior is not then merely a device to draw one's attention to something, such as might be done by turning another person's head in a certain direction, but has a genuine, though minimal, sign status; it is a preparatory-stimulus influencing the orientation of behavior with respect to the location of something other than itself. ${ }^{\mathrm{D}}$

Three kinds of identifiors may be distinguished. Indicators are identifiors which are non-language signals. The pointing gesture and the weather-vane may serve as examples. Such an indicator as pointing is however often unsatisfactory, since there are many regions in the spatial sector which pointing isolates, and since the region to be identified may not fall within the immediate environment of the organism. Language meets this difficulty by the development of descriptors, identifiors which describe a location. 'At ten o'clock tonight,' 'at the corner of 23rd Street and Broadway,' 'at the intersection of $x=3$ and $y=z$ on graph $A^{\prime}$ are examples. Such descriptors themselves involve signs in the other modes of signifying and may need (perhaps always need) supplementation by indicators to complete the identification. Since it is difficult to see that such descriptors always substitute for other signs they may also be signals (language signals). Namors are identifiors which are language symbals, and hence are substitute signs synonymous with other identifiors. In locating a person by pointing, a 
sound may be uttered which becomes synonymous in signification with the particular act of pointing; henceforth behavior may be directed to a certain space-time region by uttering this "name." Or the intersection of lines on a graph may be named, say by ' $B$ '; ' $B$ ' acts henceforth (at least for some time) as an identifior of this region. Terms such as 'it,' 'this,' 'I,' 'now' are likewise namors, singular signs like "proper names" but differing from proper names in that what they denote varies with the circumstances of production of the individual sign-vehicles of the sign-families to which they belong.

In contrast to identifiors, designators signify characteristics (discriminata) but do not determine their location. 'Black,' 'deer,' 'taller,' appearing in sign-behavior, dispose the interpreters to responsesequences which would be terminated by something black, by a deer, by something taller than something else, but they do not give temporal or spatial orientation to such response-sequences.

It is necessary to be more specific as to what is intended by 'discriminatum' in order to sharpen the distinction between designators and appraisors or prescriptors. ${ }^{\mathrm{E}}$ By a discriminatum is meant a characteristic of an object or situation which discriminates it from other things. A discriminatum is a characteristic in that it distinguishes some object as a stimulus-object of a certain kind; it is not necessary that the stimulus-object actually affects a sense-organ or even that it can directly affect a sense-organ, but it must be such that it can have direct causal effects. A book which completely designated an object (say Africa) would signify all its discriminata, but this complete designation would not include the book itself, that is, it is not a characteristic of Africa that books are written about it. An object then may have properties which are not discriminata. Africa may be written about, liked, disliked, damned; these are not characteristics of Africa but of someone's response to Africa. Discriminata belong to the stimulus side of a stimulus-response situation; what an organism can observe about an object or situation belongs to (though it does not exhaust) the discriminata of the object or situation. Designators, as signifying discriminata, signify to an organism the characteristics of what it will or might encounter; they do not signify the import of this object for its goals or what response-sequences are required toward the object 
in order to reach its goals. Appraisive and prescriptive ascriptors may also be designated, but as designated they are not themselves appraised or prescribed.

Designators may be classified in terms of the number of identifiors they require for the completion of ascriptors in which they appear. 'Black' is in this sense monadic, 'hits' dyadic, 'gives' triadic. Designators may also be distinguished in terms of the number of characteristics or the special kind of characteristics which something must have to be a denotatum. One distinction of interest is that between objectdesignators and character-designators. 'Deer' and 'black' are both monadic, since we can say 'that is black' and 'that is a deer.' But it would often be added that 'deer' designates an object and 'black' a quality of an object. The difference seems to consist in the requirement that object-designators signify a complex of characteristics which must endure over a certain time. Terms such as 'object' and 'thing' are however very vague in common usage, especially in the range of endurance which they require in order to denote. So the distinction is not of great importance in semiotic; it is enough to show that it can be made, and made without either affirming or denying a "substance-accident metaphysics." It should be remarked that object-designators do not coincide with "nouns," since 'black' can as well be a noun as 'deer.'

It has often been maintained by semioticians that identifiors must by their nature denote. In our terminology this is not required. Magicians frequently "point" to things which do not exist where pointed to, a descriptor signifying the intersection of two streets may continue to signify when in fact the streets no longer exist, and an object named may no longer exist or may not have yet come into being. Because of these and other considerations it is preferable to have a terminology in which 'denotation' is never an implicate of 'signification,' that is, in which no sign necessarily denotes. This terminology will be extended to ascriptors as well as to signs which occur in ascriptors.

A designative ascriptor is a complex sign involving identificative and designative modes of signifying: what is identified by the identifior or identifiors is further signified by a designator or designators. Designative ascriptors correspond roughly to what are often called "state- 
ments." We shall however employ the term statement for the production of a designative ascriptor by some interpreter. A designative ascriptor is then an abstraction from a statement, that is, is only the signification involved in a statement. The fact that someone states the ascriptor (produces it, asserts it, believes it) is, following H. M. Sheffer, distinguished from what the ascriptor signifies. Analogous distinctions will be made in the case of other kinds of ascriptors.

\section{Appraisors and Appraisive Ascriptors}

Organisms, given certain needs, prefer certain objects to others. Such preferential behavior is a widespread and perhaps universal characteristic of living systems. So it is natural that it should be reflected in sign-behavior. It is believed that such preferential behavior gives the behavioral clue for the interpretation of appraisive signs. We have previously defined an appraisor as a sign which signifies to its interpreter a preferential status for something or other, that is, which disposes its interpreter to favor or react unfavorably toward this something or other. Therefore the test whether a sign is or is not an appraisor is given by determining whether or not the sign disposes its interpreter to preferential behavior to something or other. ${ }^{F}$ To the extent that the sign is only appraisive it will not signify in the other modes and hence will require supplementation by other signs; frequently, however, a sign which is appraisive is also designative or prescriptive, and our test permits the determination of the extent to which this is so.

If we call the preferential status which objects have in behavior valuata, then appraisors may be said to signify valuata. The appraisor is a sign since it exercises a control over behavior of the sort which certain objects would exercise if they were present. If a chimpanzee preferred bananas to lettuce, and if it responded differentially to two sounds each of which designated food in a different place when a third sound was combined with one or the other sounds in a stimulus complex, the third sound would be an appraisor since it would cause the kind of preferential behavior shown to bananas and lettuce when they were both present. It is believed that such signs as 'good,' 'better,' 
'best,' 'bad,' 'worst' operate at the human level as fairly well-differentiated appraisors. Many signs which are designative also have a recognizable appraisive element (such as 'honest,' 'thief,' 'coward,' 'inconsiderate'). And appraisive signs are frequently prescriptive.

Consider for instance the terms 'preferable' and 'preferred.' ' $A$ prefers $B$ to $C$ ' is a designative ascriptor, signifying certain characteristics of $A$ 's behavior; it does not itself signify that $B$ is better than $C$ (does not do so if its interpreter is not in his behavior disposed because of signs to act preferentially to $B$ ). Similarly 'sign $X$ is an appraisor to $Y$ ' is a statement about sign $X$ and not an appraisal. But the utterance ' $B$ is preferable to $C$ ' is more complex. It certainly may have an appraisive element (' $B$ is better than $C$ ') and a prescriptive element (' $B$ ought to be preferred to $C^{\prime}$ ), but it also may have a strong and even dominant designative core: ' $B$ gives more complete satisfaction to some need or needs than does $C$.'

Now if we take 'preferable' to have this designative signification, then an animal may prefer objects which are not preferable, that is, do not as fully satisfy its needs as would other objects. And this is often the case. For while there is a general tendency for organisms to come through trial and error to prefer what is preferable with respect to the satisfaction of their needs, at any given time there may be a wide discrepancy between what is preferred and what is preferable; in the case of psychotic behavior the tendency to correct preferences in terms of preferability is almost completely absent.

The point to be stressed is that the determination of whether or not a sign is appraisive (in terms of whether or not it disposes its interpreter to preferential behavior) must not be confused with the question of determining whether what it signifies is in fact preferable. Only the first inquiry is the task of a scientific semiotic; the second question belongs to other disciplines. It does not follow from ' $X$ is disposed because of a sign to prefer $Y$ to $Z$ ' that ' $Y$ is preferable to $Z$,' or even that ' $X$ will in fact prefer $Y$ to $Z$.' Semiotic is not then a "theory of value"; its concern with "judgments of value" is directed solely to the kinds of signs which occur in such judgments. The situation is analogous to the determination of whether a certain sentence in a 
physics textbook is or is not a designative ascriptor. Such determination belongs to semiotic, but the question whether the sentence is or is not true is the task of the physicist and not the semiotician. For the purposes of semiotic the important point is merely to distinguish the sense in which a term such as 'good' is appraisive or designative or prescriptive. 'Good' is an appraisor in so far as it disposes its interpreters to preferential behavior, and is a designator in so far as it signifies merely that some organism has in fact certain preferences or that something is preferable to something else in the sense that it more fully satisfies a certain need. We have given behavioral tests for distinguishing the cases in terms of the disposition to behavior evoked in interpreters, and that is all that semiotic as such can be expected to do.

The appraisor, when so regarded, is not a designator, for what it signifies is not discriminata; it as such neither identifies an object (though it of course may appear in a descriptor) nor characterizes an object. Valuata are not characteristics of objects-that is, are not among the possible stimulus features of objects. They may be called "properties" of objects, since 'property' is a very general term used to embrace alike the denotata of signs in all modes of signifying; but they are not characteristics of objects in the sense in which colors, weights, and shapes are characteristics. An object is preferred because of some of its characteristics, but its preferential status is not itself another characteristic; the valuata of an object are not additional characteristics of the object, some among others, acting as stimuli to behavior, but are determined by the status which this object has in behavior; its preferential statuses are its valuata, and appraisors signify such a status.

Further, valuata are not to be described in terms of the actual satisfaction of needs, even though the preferential status of an object is normally related to and controlled by the degree of satisfaction it gives to some need-for as we have seen a man or animal may be disposed because of a sign to prefer an object which does not actually satisfy its needs or does not satisfy them as well as other objects would. And an organism may not in fact accord to an object the preferential status it is signified to have. Hence appraisors, as signifying preferential status, do not designate preferences nor needs nor the capacity of an object 
to satisfy a need. Statements can be made about appraisors and statements can be appraised, but statements and appraisals are different in kind, that is, signs in different modes of signifying.

Whether the term 'good,' for example, is an appraisor can only be determined by the way it operates in specific sign-processes. If a person says ' $X$ is good' and investigation shows that he is designating simply the capacity of $X$ to satisfy some need of another person or of himself, then 'good' is not here an appraisor but a designator; if however the interpreter is disposed to accord to $X$ a preferential status in his behavior when he or others signify that ' $X$ is good,' then 'good' is to him an appraisor. In the latter case Ogden and Richards are correct in saying that in 'this is good,' "we merely refer to this, and the addition of 'is good' makes no difference whatever to our reference" "if by 'reference' is meant identification or designation, for 'this' is here the identifior and 'good' is an appraisor. But when they add that "it serves only as an emotive sign expressing our attitude to this, and perhaps evoking similar attitudes in other persons, or inciting them to actions of one kind or another," they fail to isolate clearly enough the distinctive signification which 'good' as an appraisor has. For the term 'good' does not signify an emotion and is no more "expressive" of its user than is any other sign. ${ }^{G}$ The appraisor simply signifies the preferential status of objects; it determines which objects the organism is disposed to favor in its behavior. ${ }^{\text {H }}$ But it neither adds to the designation of the objects nor does it designate (or prescribe) specific responses of a particular behavior-family to the otherwise designated objects, nor is it always expressive of actual approval or disapproval in its interpreters.

Appraisors signify along a positive-negative continuum, and so may be classified as positive or negative. An object may be signified as 'best,' 'very fine,' 'rather good,' 'fair to middling,' 'rather bad,' 'very bad,' 'worst' with many intermediate shades of graduation; a cautious man may be signified as 'wise,' 'far-sighted,' 'prudent,' 'hesitant,' 'indecisive,' 'cowardly'-terms which have a strong designative component and yet which in many instances are markedly appraisive.

What is appraised may operate in behavior either as a means-object

${ }^{6}$ The Meaning of Meaning, 5th edition, p. 125. 
(subordinate goal-object) or goal-object (superordinate goal-object). The distinction between means-objects and goal-objects is relative to a particular need, since presumably any object which itself satisfies a given need may be a means-object with respect to the satisfaction of some other need, and any object which is a means to obtaining goal-objects with respect to a given need may itself be a goal-object with respect to another need; nevertheless with respect to a given need the distinction can legitimately be drawn. Hence appraisors can also be classified as utilitators or consummators, depending upon the status in behavior of the object which they signify. This distinction, unlike that between positive and negative appraisors, is not however a distinction in terms of the significata of appraisors themselves.

The combination of an appraisive term with an identifior such that the appraisor signifies what the identifior signifies constitutes an appraisive ascriptor. An appraisive ascriptor, considered as being produced by someone is an appraisal; this fact about its production is not however a part of its signification. An appraisal is not a statement; both signify but differ in their mode of signifying. Just as appraisals may themselves be designated, so may statements be appraised. Anything which may be signified at all may be appraisively signified, whether it be an object, an organism, a relational complex, a prescription, a statement, or even an appraisal itself.

\section{Prescriptors and Prescriptive Ascriptors}

Organisms, given a need, do not merely react to certain kinds of objects rather than to others, and accord to certain objects of a given kind a preferential place in behavior; they often also exhibit a marked persistency to respond in a given situation by some response-sequences rather than others. Food behind a barrier is responded to differently than food directly available, and the response of the chimpanzee to lettuce is not identical with its response to bananas. By inheritance or by learning the animal shows a tendency to favor certain responsesequences in a behavior-family, and the situation helps to determine at a given moment the hierarchical order of the favored responses. ${ }^{I}$ By and large an animal responds with those responses to a situation 
which are suited to satisfy its motivating needs, and in proportion to its learning capacity, repeats at later times those response-sequences which have proved to be most effective in securing means-objects or goal-objects. But whether the responses are effective or not, and whether or not the more effective responses are retained in similar future situations, the basic fact is that animals in specific situations often are disposed to some ways of acting rather than others.

If the deer is to be shot, the hunter must not be detected until he is within a certain distance of the deer; and he must handle his rifle in a certain way if the bullet is to reach the mark. One action rather than another "must" be performed, "should" be performed, is "required" by the organism-environment situation, if the act is to pass on to its consummation. This requiredness of certain actions within certain situations, like all features of behavior-situations, is reflected at the level of signs, and such signs have the distinctive mode of signifying here called prescriptive. 'Come here!' 'The employment bill is "must" legislation,' 'Keep the wind ahead!' are examples. Prescriptors are signs which signify to their interpreters the required performance of a specific response to some object or situation. They are signs since they are substitutes in the control of behavior for the control some situation would exercise if it were present; they signify the requiredness of a course of behavior to certain objects; their signification is determined by finding the behavior which they as substitutes produce and which the organism is disposed to perform. Things and persons make demands upon behavior, and the signs which signify something as requiring certain responses (obligata) are prescriptors. A person may by various devices other than language signs show that he needs another person near to him (use of force, stretching out his arms, fainting); the command 'Come here!' substitutes for such devices by signifying to the interpreter the requiredness of moving into the neighborhood of the producer of the sign. In self-observation the prescriptor is accompanied by a sense of obligation to act in a certain way; behaviorally the prescriptor is isolated by finding out the persistent behavior which it induces (or tends to induce) in the interpreter consequent to its appearance.

Prescriptors, like appraisors, normally involve designation by other 
signs. They often occur in a sign-process in which objects and actions are designated and they signify a person or objects as requiring certain responses. Since the requiredness is not itself a characteristic of either the action or objects, the prescriptor is not itself a designator; it prescribes action to objects rather than describing either action or objects; it operates in the selection of one course of behavior rather than another. The prescriptor thus gives a favored position to certain responsesequences just as the appraisor gives a favored position to some objects. A sign which is a prescriptor frequently also has a recognizable appraisive element: the action prescribed is appraised as positively significant in such a term as 'ought.' Nevertheless the prescriptive component is distinguishable from the appraisive, though actions too may be appraised and appraisals prescribed. The signification of the command 'Come here!' does not lose its prescriptive character, though it may lose its efficacy, even if to the interpreter nothing is signified as to the import of the action prescribed, and even if the action in question is not in fact conducive to the realization of the goals of either the producer or the receiver of the command. The prescriptive mode of signifying remains then a distinctive mode of signifying.

As in the case of other signs, it is necessary to distinguish between a sign actually occurring in the prescriptive mode of signifying and statements about a sign as a prescriptor or about the relative effectiveness of various responses. In the former case the sign controls the behavior subsequent to its appearance by provoking a persistent tendency to perform certain response-sequences rather than others; in the latter case designation but no prescription occurs. Thus for $Y$ to say that a certain sign is for $Z$ a prescriptor, or is generally a prescriptor, or that the action prescribed by the sign does or does not meet a certain need more effectively than other actions, does not require that the sign is a prescriptor for $Y$ himself.

Prescriptors may be classified as categorical, hypothetical, and grounded. The categorical prescriptor (such as 'Come here!') signifies without qualification an action as prescribed; the hypothetical prescriptor signifies an action as prescribed under certain conditions ('If your brother phones, come here!'); the grounded prescriptor signifies not merely an action as prescribed but signifies reasons why the action 
is prescribed ('Come here so I can give you the note!'). The term 'ought' is complex in its signification; it is a general sign that the prescriptor in which it appears can be grounded, though the grounds are not themselves signified-hence to this extent it appraises the prescribed action positively. Prescriptors have various degrees of generality, as do designators and appraisors. 'Ought' and 'ought not' are analogous in this respect to 'thing' and 'nothing,' and to 'good' and 'bad.'

The combination of a prescriptive term with an identifior or identifiors such that a specific response is signified as required with respect to what is identified is a prescriptive ascriptor. A prescriptive ascriptor considered as produced by someone is a prescription. Prescriptions are analogous to appraisals and statements, but differ in the mode of signifying of the underlying ascriptor.

\section{Formators and Formative Ascriptors}

We now mention one of the most difficult topics in semiotic: the interpretation of what are often called 'logical signs' or 'formal signs' or 'syncategorematic signs,' terms which various authors apply to such features of language as 'or,' 'not,' 'some,' 'is,' '十, ' 'five,' variables, wordorder, suffixes, parts of speech, grammatical structure, punctuation devices, and the like. Here differences of opinion run riot: there is no agreement as to which terms belong to the intended class, whether these terms are properly regarded as signs, and if they are signs, whether they are designators, appraisors, prescriptors or signs in a distinctive mode of signifying. The most one can do in this preliminary section is to recast the problem into a behavioral form, postponing until later the subtleties and complexities which are involved.

Let us take some concrete examples. In Russian there is a sound pronounced 'lee,' which when added to other signs gives the sign complex the form of a question and so changes an otherwise designative or appraisive ascriptor into a prescriptive one (since it now demands a verbal response). We would say of a person, who consistently replies to sign complexes which contain this sound by verbal responses which answer questions, that he "understands the meaning of 'lee." " 
Suppose we want to determine whether a child understands parentheses in numerical expressions. If we gave the child such marks as ' $(2 \times 3)+4$ ' and ' $2 \times(3+4)$,' asked for the results, and obtained respectively the answers ' 10 ' and '14,' we would say that the child understood that the numbers combined in parentheses form a unit which is then combined with other numbers in the expression-and that this in fact gives the signification of the parentheses.

If a dog were trained to respond differentially to five food pans containing food when each of five sounds was produced, and now if a sixth sound were introduced always with some two of the other sounds, and if the dog then always went first to one of the two designated pans and to the other if and only if food was not obtained in that pan which it first approached, it might be said that the sixth sound signified to the dog what 'or' in one of its English usages signifies.

If now we notice what 'lee,' '( )' and 'or' have in common in these examples, four points seem to be relevant:

1. Some stimulus is added to signs which already have a plurisituational signification and which are signs in other combinations where the stimulus in question does not appear;

2. When the new factor is added the signification of the particular sign combination in which it appears is different from when it is absent, as is evidenced by the difference in behavior correlated with its appearance;

3. The new stimulus does not itself signify additional stimulus features of the otherwise designated situation (that is, does not determine the characteristics of objects to which the organism is prepared to respond), nor does it add to the appraisal of what is otherwise designated nor add to the prescription of how to act to what is otherwise designated;

4. The new stimulus influences the response of the person stimulated to the signs with which it appears in a particular sign combination by affecting the interpretants aroused by the other signs in the sign combination; only in that way does it affect the behavior of the person to the situation otherwise signified by the signs which accompany it.

Stimuli with such properties will be called formators. Such ascriptors as 'It will rain tomorrow in Chicago or not rain' will be called 
formative ascriptors; the production of a formative ascriptor will be called a formulization. Chapter VI will be devoted to a discussion of formators, the classification of formators, and formative ascriptors. In the meantime we will regard formators as signs in a distinctive mode of signifying; without further clarification at this point we will say that formators signify distinctive properties of situations later to be called formata. ${ }^{J}$ Whether formators are signs in the sense in which identifiors, designators, appraisors, and prescriptors are signs remains for the present an open question. We have given here merely a preliminary indication of their nature in order to round out the classification of modes of signifying, and to make some reference to formators possible in the intervening stages of our argument. The basic points relevant for the moment are that formators presuppose other signs and that they influence the signification of the specific sign combinations in which they appear. The existence of formators and formative ascriptors is not in doubt, but their interpretation raises difficult problems. Hence the advisability of postponing the discussion of these issues until we have further clarified the topics of truth, reliability, adequacy, and the main types of discourse.

\section{Interrelation of the Modes of Signifying}

The phrase 'mode of signifying' has been adopted since it suggests that the differences between signs with which we have been concerned in this chapter are differences in the ways something can be signified. For anything whatsoever may be signified with respect to its location, or its characteristics, or the preferential status to be accorded to it in some context of behavior, or the kind of response it requires if certain goals are to be reached, or the way it is signified by a combination of signs. Drops of water falling from the sky can be signified as occurring at a certain time and place, or as being rain, or as being a blessing, or as something which ought to be collected, or as something which always must either occur or not occur. All of these properties may be relevant to behavior, but not all of them are signified nor need to be signified in every sign-process. For behavior does not always have the clues 
which signs might advantageously afford, and often the situation makes unnecessary signs in all modes of signifying.

The modes of signifying are therefore in one sense independent, since signs in one mode may occur without there being signs in the other modes. To take an example at the level of language communication, one person might supply cues to another person's behavior by simply saying 'there,' 'rain,' 'good,' 'must,' singly or one after another in any order, depending on the situation. And the same would hold for ascriptors 'That is rain,' 'That is good,' 'You must do it'; one can be - used without the other, and when a number are used they may be produced in any order. It is possible to signify that something requires a certain response without signifying what the something is, or to signify the characteristics of something without signifying its location or its import.

There is nevertheless a sense in which the modes of signifying are interdependent. When the situation does not itself supply the clues needed for the direction of behavior, the organism or other organisms may supplement the situation by signs. And if signs in a number of modes of signifying are produced, these do depend on each other in certain ways. The prescription of an action is not of much help under these circumstances unless the object to which the action is to be directed is appraised, designated, and identified. There is a sense then in which in a highly problematic situation where behavior needs full direction, prescriptions require appraisals and appraisals require statements to a degree to which statements do not require appraisals or appraisals require prescriptions. To put the same point in another way, an organism which requires direction by signs must as a minimum have such signs as direct its behavior to the kind of objects it needs and their location; it can then try out their relevance and how to act on them in case these objects are not further signified appraisively and prescriptively. But the organism would often be quite helpless in its behavior if it merely had a sign that something was good or must be treated in a certain way without this something being designated, for behavior would then be without orientation. For this reason prescriptions rest on appraisals and appraisals on statements in a way in which 
statements do not need to be followed by appraisals and appraisals by prescriptions. These interrelations will become more evident when we consider the relations of such types of discourse as are found in science, art, religion; in that context we will also consider the dependence of formators upon signs in the other modes of signifying.

The order of dependence of signs in the various modes of signifying does not of course mean that in genesis identifiors, designators, appraisors, prescriptors, and formators appeared in a corresponding temporal order. As has been stressed before, the early sign situations involve these components in an undifferentiated form, and their progressive differentiation may well be an essentially simultaneous process. As one kind of sign evolves, the differentiation of the other kinds is made necessary if sign-processes are to be complete and adequate for behavior. Signs which approach the status of pure identifiors, pure designators, pure appraisors, pure prescriptors, or pure formators come late in the process of genetic development and perhaps approach this status simultaneously. At any rate the historical development of language is a distinct problem, and our analysis does not prejudice the answer. ${ }^{\mathbf{K}}$

Aside from the interdependence of kinds of signs furnished by the requirements of activity, there are other kinds of interrelatedness. What is designated can always be appraised and can always be made the basis of a prescription. It is further possible to designate appraisals, prescriptions, and formulizations, to appraise and prescribe designations, appraisals, and prescriptions, and to combine any of these kinds of signs with formators to build more complex sign combinations. Because of this fact it is understandable that many persons should have tried to reduce the various modes of signifying to some one mode, to the designative for example, or to the prescriptive. Thus the fact that a certain sign which is appraisive or prescriptive for a given organism can itself be designated has encouraged some persons to regard designation as all-sufficient and to believe that appraisals and prescriptions are synonymous with certain statements. In a similar way, the fact that most or all signs as they actually occur have something of a prescriptive nature has led some persons to assimilate statements and appraisals to prescriptions as to how we are to behave. The preceding analysis per- 
mits us, however, to avoid these reductions by suggesting behavioral criteria for the distinction and quantitative analysis of the various modes of signifying, and since these criteria are different, signs in the various modes of signifying, while interrelated, cannot be identified with each other, a point important for understanding both the distinctive nature and the interrelations of such cultural phenomena as science, art, religion, mathematics, and philosophy. 


\section{ADEQUACY, TRUTH, AND RELIABILITY OF SIGNS}

\section{The Sense in Which a Sign Is Used}

TN THE preceding chapter the modes of signifying were distinguished from the standpoint of the interpreter. The difference between designators, appraisors, prescriptors, and formators was put in terms of differences in the behavior they prepared. To say that a sign is in one or the other modes of signifying is to say that the sign operates for some interpreter in one or another of the ways described, or generally so operates for some set of interpreters. No account was taken of the manner in which the sign appeared, that is, whether the sign was an event in the non-social environment, whether it was produced by other organisms, or whether it was produced by the organism which interpreted the sign. Attention must now be turned to one phase of the production of signs: to the question of the purpose for which an organism produces the signs which it or other organisms interpret. We now look at signs not from the point of view of their interpretation but in terms of their relation to the purposive behavior in which they are produced and which they serve.

A sign $S$ will be said to be used with respect to purpose $y$ of an organism $z$ if $y$ is some goal of $z$ and if $z$ produces a sign which serves as means to the attainment of $y$. If a person is seeking money and writes a short story to achieve that end, the sign complex which is the short story is "used" for the purpose of getting money. The usage may 
be voluntary or involuntary, however these terms are defined. If by voluntary, for instance, is meant action performed as a result of signifying to oneself the consequence of performing the action, then the production of signs may be voluntary (as where one writes a short story after signifying that the short story is a way to get money) or involuntary (as where one writes a story to depreciate a certain person without having signified to himself that this is the purpose the story is to achieve). In the present state of behavior theory such distinctions often cannot be easily made with much reliability, and they will not figure prominently in our account. To know that a sign is even used in the sense defined is also often difficult, but less so: there is seldom doubt that a chimpanzee is using a stick to pull a banana into its cage, and in the same sense there is often little doubt that an author writes to gain money. ${ }^{A}$

A sign is adequate to the degree to which it achieves the purpose for which it is used. Signs adequate for some purposes may be inadequate for others. To say that a sign is adequate is to say that its use reaches a goal in a particular occasion or that in general it facilitates the attainment of a certain goal. An understanding of the kinds of adequacy which signs have is dependent then upon an understanding of the uses to which signs are put.

The classification of the uses of signs is made difficult by the fact that almost every need which an organism has may utilize signs as means to its attainment. Signs may serve as means to gain money, social prestige, power over others; to deceive, inform, or entertain; to reassure, comfort, or excite; to record, describe, or predict; to satisfy some needs and to arouse others; to solve problems objectively and to gain a partial satisfaction for a conflict which the organism is not able to solve completely; to enlist the aid of others and to strengthen one's own independence; to "express" oneself and to conceal oneself. And so on without end.

Various attempts have been made under these circumstances to attain some simple classification which will embrace at least the majority of the ways in which signs are used. The most frequent of these attempts repeat or stem from the Ogden and Richards' distinction between referential and emotive. Pollock distinguishes the referential and the 
evocative uses of signs; Mace the referential and expressive; Reichenbach the cognitive and the instrumental; Feigl the informational and the non-cognitive; Stevenson the cognitive and the dynamic. The second member of each pair is often further analyzed into subordinate usages: such as into feeling, tone, and intent (Richards); communicative, suggestive, promotive (Reichenbach); imaginative, affective, directive (Feigl). ${ }^{\mathrm{B}}$ These attempted classifications often confuse the signification and the uses of signs. But in spite of this they obviously have many points in common, and suggest that the primary uses of signs correspond in some sense to the modes of signifying which we have isolated. Designators for instance are especially adapted to the primary purpose of giving information, and yet they may be used to gain an income; while over and above their primary and secondary uses they may have a number of individual and social effects or consequences which they are not intended to attain.

An analogy may help to make clearer the differences between a kind of sign, its primary and secondary uses, and the further consequences of its existence. A gasoline engine is to be distinguished from a steam engine in terms of its mode of operation. Each type of engine has, further, certain functions which it is normally used to perform, and these are its primary uses. One type of engine may however under certain circumstances be used to perform the task for which another type of engine is normally employed, and this would be among its secondary uses. Other secondary uses would be to increase the income of the owner of a machine, or to serve in an exhibit of various mechanical appliances. The full story of engines would, however, even go beyond a description of how they work and their usages: it would include the effects of engines upon the men who design, build, and operate them, and upon the whole cultural complex in which they appear. The case of signs is parallel: kinds of signs may be distinguished in terms of what they signify, their primary and secondary uses considered, and the effects of their existence upon the users of signs and the cultural complex in which they operate studied. Semiotic is concerned with all these matters, but it must not confuse them. For the moment we limit attention to the primary uses of signs in the different modes of signifying. 


\section{The Four Primary Sign Usages}

Signs in general serve to control behavior in the way something else would exercise control if it were present. To attain its goals the organism must take account of the environment in which it operates, select for its concern certain features of this environment, respond by response-sequences which will attain an environment suitable to its needs, and organize its sign-provoked responses into some pattern or other. Each of these stages of its activity may be facilitated by the use of signs, and the four primary usages of signs correspond to these four aspects of behavior.

Signs accordingly may be used to inform the organism about something, to aid it in its preferential selection of objects, to incite responsesequences of some behavior family, and to organize sign-produced behavior (interpretants) into a determinate whole. These usages may be called in order the informative, the valuative, the incitive, and the systemic uses of signs. These are the most general sign usages; other usages are subdivisions and specializations of these four. They are the purposes for which an individual produces signs as means-objects in the guidance of his own behavior or in the guidance of the behavior of others. They may be employed with respect to things other than signs or to signs themselves.

An individual may use signs to inform himself or others about what has been or is or will be, with respect to signs or non-semiosical events. He may use signs to confer for himself or others a preferential status upon something-upon things, persons, needs, or even signs (as where he wants the signs he himself produces to be approved as "fine writing" or "fine speech"). He may use signs to incite a particular response in himself or in others to objects or signs, or to call out submission in some one else, or to get the reply to a question which bothers him, or to provoke co-operative or disruptive behavior in the members of some community. And he may use signs to further influence behavior already called out by signs, whether this behavior be to signs themselves or to something other than signs. It seems, indeed, that all the uses to which signs may be put can be classified under these four heads, whether the ends they subserve be further specialized as entertainment, 
domination, co-operation, reassurance, deception, instruction, or the like.

It is obvious that these four comprehensive usages of signs are closely related to the four modes of signifying. The primary use of designators is informative, the primary use of appraisors is valuative, the primary use of prescriptors is incitive, and the primary use of formators is systemic. So close in fact is this relation that doubt may be raised as to whether the mode of signifying of a sign can be distinguished from its corresponding primary usage. Thus if a sign is for a particular person at a given moment a designator, it is informing him (correctly or incorrectly) about something, and if a sign is for a particular person at a given moment an appraisor it tends to confer a preferential place in his behavior to something. Hence it may seem, for instance, that the designative mode of signifying is not distinguishable from the informative use of signs, and that the modes of signifying may perhaps even be defined in terms of the primary uses to which signs are put.

That the distinction is, however, valid and important may be made clear by the following considerations. The term 'use' (or 'function') has various significations. If no more is involved than the position that all signs are to be distinguished in terms of differences in behavior in the situations in which they occur, then of course the occurrence of a sign cannot be distinguished from its "use." We however have defined the use of signs in terms of the production of signs as means-objects to accomplish some end, and in this more limited sense a sign-process can occur without being used-thus a designator may give information without being used to inform. For a sign may be designative without being produced by any organism and an organism may interpret a sign produced by another organism without itself producing (or using) the sign itself. Then too it must not be forgotten that to call a sign designative may mean either that it is designative for some interpreter at a given moment or that it is in general designative for the members of a given community. Hence a person may produce a sign which is generally designative in order to inform someone else of something, and yet it may happen that the sign does not operate in that process as a designator for the someone in question. Here a sign used to inform someone is not in fact for the person addressed a designator. Anal- 
ogously, a person may aim to inform someone else about himself without using signs designating himself; he may for instance write a poem praising the moon and hope that the reader will regard the production of the poem as an expressive sign signifying something about the author; here the production of appraisors is used to accomplish the goal of conveying information. For such reasons it is wise to distinguish the mode of signifying of signs from the uses to which they may be put, even though it is true that each kind of sign is primarily (and in general, most adequately) used for the accomplishment of a certain purpose.

Corresponding to the primary usages of signs are various kinds of adequacy. There are no satisfactory terms in common employment to mark these distinctions. 'Truth' is often a synonym for the adequacy of a sign, but since the term frequently blurs the distinction between denotative reliability and adequacy, it had best be avoided as a synonym for 'adequacy.' We shall somewhat arbitrarily call a sign that is informatively adequate 'convincing'; 'effective' will be used for valuative adequacy, 'persuasive' for incitive adequacy, and 'correct' for systemic adequacy. $\mathrm{C}$ discussion of these types of adequacy will supply an opportunity to expand the treatment of sign usage, and to consider the relations of such usage to the topics of truth, knowledge, and belief.

\section{Informative Adequacy: Convincingness}

In the informative use of signs, signs are produced in order to cause someone to act as if a certain situation has certain characteristics. If food is present in a certain place, then to produce signs so that a dog will behave to the given pan as containing food would be to use these signs informatively, that is, to inform the dog that food was in the pan in question. Signs may be used by one organism to inform other organisms or to inform itself, as where one makes a note of something observed in order to inform oneself at a later time of what was observed. In the informative use of signs the producer of a sign seeks to cause the interpreter to act as if some present, past, or future situation had such and such characteristics.

The information which is thus given may be of various kinds. Signs 
may be used to inform someone about the physical environment, or about certain needs, or how certain objects are related to the satisfaction of needs, or the preferential status which certain objects have for certain organisms, or what someone holds to be desirable, or the characteristics of certain signs. Insofar as signs are used to cause an interpreter to act as if something has certain characteristics they are used informatively.

Signs in any of the modes of signifying may be used informatively. A sign which is in a given community normally an appraisor may be produced by a given person in order to cause someone to act as if the producer himself gives to objects the preferential status signified by the appraisor. Or one may utter a command to inform someone that one wishes a certain action to be performed. Or various grammatical forms may be produced to inform someone how signs are combined in the language in question. In all these cases information is conveyed by the use of appraisors, prescriptors, or formators, and yet that about which information is conveyed is not itself designated by the signs produced, that is, the signs produced are not designators.

Nevertheless, designation is always to some extent involved in the informative use of signs, since the interpreter of the sign must interpret the production of the sign in question designatively even when the sign produced is not itself a designator. If $A$ wants to convey information about himself to $B$ by writing a poem praising the moon, then $B$ must interpret the poem as an expressive sign if $A^{\prime}$ 's purpose is to be adequately realized, that is, must take the fact of its production by $A$ as designating something about $A . B$ of course may not do this, but may remain content with what the poem itself signifies about the moon. For such reasons there is a strong tendency to use directly designative signs where the adequate conveying of information is the main goal sought. Designators do always inform, and hence it is natural that their primary use should be the informative one, even though it remains true that they may be used for other purposes and that other kinds of signs may be used informatively.

A sign is informatively adequate (or convincing) when its production causes its interpreter to act as if something has certain charac- 
teristics. Since such convincingness is a matter of the use of signs, it is not to be confused with the question of the denotative reliability of the signs employed: to inform someone convincingly of something is not necessarily to inform him truly. $A$ may convincingly inform $B$ by a poem about himself in the sense of causing $B$ to act toward $A$ as a certain kind of person, without $A$ being in fact such a person. The term 'inform' is frequently limited to those cases where the sign is not only adequate, but is "true", that is, 'inform' is contrasted to 'misinform' as 'conveying true information' is contrasted to 'conveying false information'. For our purposes it is convenient to distinguish the informative use of signs (and hence convincingness) from the question of the truth or falsity of the signs used; hence to 'misinform' a person der liberately or unknowingly is still, in this usage, to inform him. ${ }^{\mathrm{D}}$ Signs may be informatively adequate even if the signs in fact denote nothing.

It follows that the test of informative adequacy is simply whether one person by the production of signs causes another to act toward something as having the characteristics which the first person wishes him to believe it has; the signs produced by the first person for this purpose may or may not themselves be designators. Designators are normally the best signs for this purpose, but signs in all the modes of signifying may be informatively adequate, and in some cases nondesignative signs may better accomplish informative purposes than designators. The convincingness of signs is ultimately determined by seeing whether their production by one organism causes other organisms to respond to something as having the characteristics which the producer of the signs intends to convey.

\section{Valuative Adequacy: Effectiveness}

The use of signs to cause preferential behavior to certain objects, needs, preferences, responses, or signs is to use signs valuatively. The same purpose may be achieved by various other methods; we are here interested only in the use of signs for this purpose. The interpreter that is being influenced may be the producer of the signs himself or other organisms, and the preferential status which the signs are used to 
induce may be one which has already been accorded by the organism or may itself be built up as a result of a complex sign-process.

Signs in any of the modes of signifying may be used valuatively. $A$ may attempt to cause $B$ to give a preferential status to something or other merely by designating the something in question-by stating, for instance, its relation to certain needs of $B$ in the hope that $B$ will then accord the status in question to what is designated in virtue of its effects on his needs. Or $A$ may demand by prescriptors that $B$ give to the something the preferential status intended. Even formators may occasionally be used in this connection, as when $A$ presents to $B$ certain formatively signified arrangements of signs in the hope that $B$ will then adopt such arrangements in his own sign-producing behavior. Appraisors, however, are the most natural kinds of signs to use valuatively, since if a sign is an appraisor to its interpreter it does in fact dispose its interpreter to give a preferential status to what is signified. Therefore $A$ may use signs which to $B$ are appraisors in order to induce the desired preferential behavior, or may at least use signs which in the linguistic community to which they both belong are normally appraisive, hoping that such signs will in the particular instance of their appearance call out in $B$ the preferential behavior which they normally evoke in members of this community. In reading a poem in which suffering is presented as of great positive significance, the reader (as a member of a linguistic community) signifies suffering as having such significance. If he himself accords to suffering the importance signified, he may deliberately seek poems of this kind as a way of evoking, strengthening, and integrating his own attitudes; if such is not the case he, as a reader, nevertheless participates for a moment in a sign-process in which suffering is signified as having positive significance. It may or may not result that he henceforth in his overt behavior gives to suffering the significance signified. $A$ may present $B$ with such a poem in order to determine the place suffering will be accorded by $B$, or $B$ may himself seek out or write such poems in order to call out or to create in himself an attitude of a certain sort.

The degree to which a sign gives to something the preferential status it is used to establish measures its effectiveness or valuative adequacy. 
The effectiveness of various kinds of signs will vary with individual and social circumstances. If, for instance, the appraisors of a community at a given time have lost much of their interpersonal character, then the establishment of preferential behavior may perhaps be done most effectively by the use of primarily designative signs.

Effectiveness is a different kind of adequacy than convincingness, yet in various ways it often depends on the latter. For signs may not be valuatively adequate unless they or other signs convincingly communicate the where and what of that to which it is desired to cause preferential behavior. And since the preferential status accorded to things is linked-though often remotely-with the way in which they satisfy needs of the organism, the effectiveness of signs is often dependent upon the denotation (and not merely the convincingness) of certain designators, for if certain objects appraised as significant do not in fact meet the needs of their interpreters, then the appraisors may well lose their effectiveness.

An individual may use signs valuatively with respect to himself as well as to others. The process of determining what preferential status will be accorded to something or other (whether objects, needs, preferences, responses, signs) may be called, insofar as it is carried out by signs, evaluation. The signs used in evaluation may be signs in any mode of signifying, but the process eventuates in the establishment of appraisive ascriptors. Evaluation is not itself restricted to the valuative use of signs, but the appraisors which result may then in turn be used valuatively. Evaluation is itself only one of the ways in which the preferences of organisms are formed, just as the valuative use of signs is only one of the ways in which something or other is given a preferential place in behavior. The adequacy of evaluation rests ultimately on the relation of something to some need of the organism, while the valuative adequacy of signs is restricted to the specific question as to whether they are effective in achieving the purpose of inducing some organism to accord to something or other a desired preferential behavior. An effective sign in this sense of the term may or may not be positively evaluated, that is, given a preferential status with respect to other needs and purposes. 


\section{Incitive Adequacy: Persuasiveness}

In the incitive use of signs, signs are produced in order to determine how the interpreter of the sign is to act to something, that is, to call out more or less specific responses. The aim may be merely to limit the responses to those of some behavior-family or to incite some particular response-sequence within a behavior-family: thus $A$ may wish $B$ to come to him and may or may not specify the particular manner in which he is to come. In the incitive use of signs the aim is to direct behavior into definite channels, and not merely to give information or to determine the preferential status of something or other.

Once again, signs in all the modes of signifying may be used for incitive purposes. One may merely designate the consequences of performing a certain action or the relative efficacy of one mode of action to another in reaching a certain end, hoping that the desire of the interpreter for such consequences or for efficient ways of gaining them will cause him to act in the intended way. Appraisors can be used to signify a preferential status for the desired way of acting or for the need which is served by such ways of acting, in the hope that the intended action may thus be performed by the interpreter. Even formative ascriptors may be produced as a means of causing the interpreter himself henceforth to produce signs according to the formula in question. But since prescriptors are signs which do tend to invoke determinate responses, they are the signs primarily used for incitive purposes: $A$ will most frequently present $B$ with signs which are commonly prescriptive in the linguistic community of which they are both members in the expectation that the signs will in the particular case in question cause $B$ to act in the intended way. As a member of the linguistic community, $B$ will have aroused in himself the tendencies to action which the signs normally provoke; whether he himself will actually respond in the intended way will depend upon many factors, such as the needs operative in him at the given moment.

The incitive adequacy of signs has been called persuasiveness. The relative persuasiveness of different kinds of signs will vary with circumstances. Normally prescriptors are highest in persuasiveness, since 
they do tend to invoke specific actions. But in a time where the customary ways of acting have become uncertain, it may well be that signs other than prescriptors have the greater persuasiveness: it may be more persuasive to describe the consequences of a certain action than to command the action directly. The relation between the producer and the interpreter of the sign is also relevant. A parent may be able to control the behavior of a young child by a command which would be unsuccessful when used with an adult rival.

We have seen that the appraisor frequently involves designators, and that the prescriptor frequently rests on designators and appraisors. For this reason the persuasiveness of a prescriptor often depends upon the ability to convince the interpreter one is trying to influence that certain designators are reliable and that certain appraisors coincide with the preferential status which he himself accords to what is designated. The process of persuasion may therefore require the adequate use of designators and appraisors. Persuasiveness is thus often connected with the convincing and effective use of other signs: often the interpreter must be adequately informed and certain preferential statuses adequately established in order for the signs used to be persuasive. Often the preliminary situation is prepared, as in the case of the soldier trained to obey commands without hesitation. But in other cases this is not so, and persuasion can only be effected if certain needs are first made operative in the interpreter by adequately using designative and appraisive signs. It may even be necessary to cause the interpreter himself to undergo a complicated process of evaluation before the signs with which he is confronted become persuasive (incitively adequate).

A person may use signs incitively with respect to himself as well as to other persons. The process is not essentially different in the two cases, though in inciting behavior in oneself the signs used need not be linguistic signs. A person may hang on his wall a motto to incite recurrently a certain kind of behavior in himself or he may go through a complex process of evaluation before he is able to provide himself with signs persuasively adequate, as in the case where decisions have to be made which determine the whole direction of his life. Here too he must often first find true and convincing designators and effective ap- 
praisors before he attains the convictions and the commitments which permit the appearance of chosen lines of actions, and prescriptors which then persuasively incite the course of behavior which he has chosen.

\section{Systemic Adequacy: Correctness}

The systemic use of signs is the use of signs to systematize (organize) behavior which other signs tend to provoke. The limitation to signbehavior distinguishes the systemic use of signs from other attempts to organize behavior. One may, for instance, attempt to inculcate in a person by religious discourse a certain need as basic in his system of needs, and in this way to organize or systematize the needs and behavior of the individual, but such a use of signs would be incitive and not systemic as the term is here used. In the systemic use of signs the aim is simply to organize sign-produced behavior, that is, to organize the interpretants of other signs. This may be done with respect to all kinds and combinations of signs, and by the use of signs in the various modes of signifying.

Suppose that there are two signs, $S_{1}$ and $S_{2}$, which designate respectively the presence of food in two places. Then a particular relation between the two interpretants of these signs (the relation, say, of exclusive alternation) may be set up by various sign devices. One might say to someone: "The signs $S_{1}$ and $S_{2}$ are so related in the present situation that if one does not denote then the other denotes, but not both." Or one might say: "It is good when signs $S_{1}$ and $S_{2}$ both occur to seek food in one of the two designated places, and if (and only if) food is not found there to seek food in the other place." Or an action might be prescribed: "When $S_{1}$ and $S_{2}$ occur together, seek food in one of the two designated places and seek food in the other if (and only if) food is not so found." Finally, one may simply say, by the use of the formator 'or,' " $S_{1}$ or $S_{2}$." In these cases signs in different modes of signifying are all used to organize in the same way the sign-behavior (the interpretants) caused by $S_{1}$ and $S_{2}$. Since formators by their very nature affect the interpretants of the signs with which they are combined, they are especially economical and trustworthy signs for the systemic use. But they are not the only signs that may be used in this way. 
Just as formators presuppose signs in the other modes of signifying, so does the systemic use of signs minister to the other uses. Especially does it serve as a way of increasing the adequacy of other signs. A statement can often be made more convincing by linking it with designators which already function adequately, showing that it is an implicate of statements which are themselves convincing. If an interpreter is convinced by statements $X$ and $Y$, and if it can be shown that statement $Z$ is implied by $X$ and $Y$, then the chance that $Z$ will be convincing is greatly strengthened. Similarly, if $X$ and $Y$ are shown to imply a $Z$ that is convincing to the interpreter, then $X$ and $Y$ are made more convincing to the interpreter in question. Thus by the use of formative ascriptors such as occur in deduction, to relate statements systemically, the informative use of signs is often rendered more adequate. Later we will examine how formative ascriptors are especially serviceable in this connection. Here it is only necessary to call attention to the fact, and to stress the point that such ascriptors serve not only the informative use but also the valuative and incitive uses. An appraisor may more effectively be used to induce a desired appraisal if it is shown to be implied by appraisors that are antecedently effective, and a prescriptor may be made more persuasive by showing that it is a special instance of a prescriptor which is already persuasive to the interpreter one is attempting to influence.

Insofar as signs are systemically adequate they are called (though not very appropriately) correct. Such adequacy not merely influences the other kinds of adequacy, but is itself dependent upon them. Systemic adequacy may require a long preliminary process in which the person addressed must be stimulated by convincing, effective, and persuasive signs in various modes of signifying before engaging in the intended organization of his sign-behavior. The various kinds of sign adequacy are thus interrelated and interdependent. Each may lend its support to the others and inadequacy in one respect may lead to inadequacy in any other respect. And just as signs in all the modes of signifying are frequently necessary to the adequate control of behavior, the use of signs to control the behavior of an interpreter frequently requires that all of the four primary uses of signs be present and adequately performed. The modes of signifying and the primary uses of signs 
are each interdependent in their respective domains and each domain is interlocked with the other.

\section{T-Ascriptors and "Truth"}

As it stands the preceding account is obviously incomplete. For the adequacy of signs has been explained solely in terms of whether the signs produced by some organism influence the behavior of their interpreter in a way which realizes the aims of the producer of the signs. But this is not the full story. The convincingness of a sign is not the same as its truth or reliability; an effective sign may not give a preferential status to objects which actually satisfy the needs of its interpreter; a persuasive sign may incite behavior which does not in fact efficaciously reach the goals of its interpreter; a systemically correct sign may not appropriately organize behavior. In all these cases there seems to be some underlying "factual" components which are distinguished from adequacy as that term has been hitherto used, and which in some sense affect the adequacy of the signs. These neglected factors are the truth and reliability of signs.

A sign is said to be reliable to the degree that it denotes in the various instances of its appearance. If an animal always found food at a certain place when a buzzer sounded the buzzer would be completely reliable; if food were found only 70 per cent of the times the buzzer sounded, the buzzer would be 70 per cent reliable. Now since the buzzer is in this example an ascriptor, the notion of denotation must be stated in a form applicable to ascriptors.

An ascriptor will be said to denote if what is identified in the ascriptor is denoted by the dominant designative, appraisive, or prescriptive signs which complete the ascriptor. The denotatum of an ascriptor is then simply a situation such that the denotatum of an identifior (or the denotata of the identifiors) is a denotatum of the dominant sign. The buzzer sound denotes if at the place and time identified there exists the food which is signified; the ascriptor formed by pointing to a person and saying 'poet' denotes if the person pointed to is a poet (that is, is a denotatum of 'poet').

The denotation of an ascriptor is not to be confused with its reliability. 
For a particular buzzer sound may in fact denote and yet be unreliable in the sense that in general when the buzzer sounds no food exists in the place signified; conversely the buzzer sound may be highly reliable and yet in a given instance not denote.

We will call an ascriptor which denotes a $T$-ascriptor. A T-designative ascriptor is a designative ascriptor such that what is identified has the characteristics it is signified to have; a $T$-appraisive ascriptor is an appraisive ascriptor such that what is identified is accorded the signified preferential status in the behavior of its interpreters; a $T$-prescriptive ascriptor is a prescriptive ascriptor such that what is identified evokes the responses in its interpreters which are signified as required; the criterion of $T$-formative ascriptors will be given in a later discussion of formative ascriptors. That various kinds of ascriptors may be T-ascriptors follows from our account of the modes of signifying. Since discriminata, valuata, obligata, and formata were introduced as properties of objects, the various kinds of ascriptors can be said to denote if what is identified has the properties it is signified to have.

The term 'T-ascriptor' has been chosen because it is believed that ascriptors which denote would ordinarily be called "true." ${ }^{\mathrm{E}}$ Henceforth we will refer indifferently to ascriptors which denote as $T$-ascriptors or as true (and to ascriptors which do not denote as F-ascriptors or as false). Strictly speaking, however, it is only the term 'T-ascriptor' which has here been introduced into semiotic; the reader may (as in the case of 'lansign' and 'language sign') employ 'true' in any way he wishes, or avoid it altogether. 'True' is a very ambiguous term in ordinary speech, and the identification of it with 'T-ascriptor' accords with only one of its many significations. Many logicians, for instance, have since the time of Aristotle restricted 'truth' to designative ascriptors; they would not regard appraisive or prescriptive ascriptors, or such formative ascriptors as 'Come or don't come!' as true. Our terminology permits this restriction. On the other hand it does equal justice to those persons who have emphasized the similarity of appraisals and prescriptions to statements in the matter of denotation, a similarity reflected in the very common tendency in everyday speech to reply to appraisals and prescriptions by 'That is true' or 'That is false.'

The important point for semiotic is not to analyze the various signi- 
fications of 'true' nor to prescribe one of these significations but to clarify the similarities and the differences between ascriptors. The term 'T-ascriptor' does both, for it permits all forms of ascriptors to be alike with respect to denotability and yet makes clear that a T-designative ascriptor differs from other ascriptors in alone signifying characteristics of objects and situations. And since signs may have varying degrees of reliability without being T-ascriptors, room is left for the comparison of various kinds of ascriptors with respect to reliability. It is harder on the whole to obtain highly reliable appraisals and prescriptions than statements (partly because of the variability of needs from person to person and in a given person from time to time); we suspect that this is one reason why many persons are unwilling to extend the term 'truth' to appraisals and prescriptions. Our terminology does justice to both the similarities and the differences between various kinds of ascriptors, and rescues semiotic from the clamor of those who assert that "science alone is true" or "art alone is true" or "religion alone is true."

\section{Belief and Knowledge}

An ascriptor may be a T-ascriptor without being believed to be a T-ascriptor or without being known to be a T-ascriptor, just as it may be a designative ascriptor or a singular ascriptor or an adequate ascriptor without being believed or known to be such. This follows in our terminology from the way 'T-ascriptor' was introduced, since the term was defined without employing the terms 'belief' and 'knowledge.' The question now is how these terms may be given a semiotical formulation, and the relation of the terms when so formulated to their customary significations.

An interpreter may be said to believe that an ascriptor is a T-ascriptor to the degree that he is disposed to act as if the ascriptor denotes. Similarly, an interpreter believes that an ascriptor has reliability $X$ to the degree that he is disposed to act as if the ascriptor in question has reliability $X$. Belief in general may perhaps be regarded as readiness to act as if something has certain properties. In any case, belief about signs (as T-ascriptors, as reliable, as adequate) is here regarded as a readiness to act as if the signs in question had certain properties. Since 
there are degrees of readiness to act under certain conditions, there are degrees of belief about the properties of signs.

An interpreter will be said to know that an ascriptor is a T-ascriptor or has reliability $n$ to the degree he has evidence that the ascriptor denotes or belongs to a class of ascriptors such that $n$ is the ratio of T-ascriptors to the total number of ascriptors in the class. Knowledge in general may perhaps be regarded as the aptitude of an organism to regulate its behavior in the light of evidence afforded by its environment. But as in the case of 'belief,' it is not necessary for the purposes of semiotic to define 'knowledge'; we are only concerned with the terms 'belief' and 'knowledge' as applied to signs. And just as belief in respect to a sign is a matter of degree, so knowledge of signs, when defined in terms of degree of evidence that a sign denotes, or is reliable, or is adequate, is a matter of degree.

A sign may be true or reliable without being believed to be true or reliable and without being known to be true or reliable. Also a sign may be believed to be true or reliable without being true or reliable. The other relations are more complex. Since belief and knowledge about signs is a matter of degree, it is clear that there may be a low degree of knowledge and a high degree of belief. A high degree of knowledge will normally, but not always, strengthen the degree of belief. Common usage is ambiguous as to whether a sign can be known to be true or reliable and yet not be true or reliable. Some usages would not permit us to say that a sign is known to be true unless it is true; yet it is not uncommon for a person to say that he "knows" his friend is dead on the basis of certain evidence when in fact his friend is not dead. I see advantages in siding with this latter usage, since then 'truth,' 'belief,' and 'knowledge' may be regarded as independent terms, neither of which is an implicate of the other. This does not deny that there are complex relations between belief and knowledge about signs, but it transforms the study of their relations into an empirical problem.

What, however, about the relation of 'truth' and 'reliability'? Are these two independent terms? They are certainly not always independent in common usage; however, as we have introduced them into the language of semiotic, neither is an implicate of the other. For an ascriptor can be a T-ascriptor without being reliable or reliable without 
being a T-ascriptor. An ascriptor is a T-ascriptor if it denotes, but it may denote and yet be similar in signification to a set of ascriptors all of which are false. Thus the buzzer sound signifies food at a certain place, say at a future time. If there is food at the place at that time, the buzzer is a $\mathrm{T}$-ascriptor. But at the time the buzzer sounds the question of its reliability is not stated in terms of the truth or falsity of this ascriptor but in terms of the proportion to which other buzzer sounds have signified food in a certain place at a certain time relative to their occurrence. These sounds may have all been false and so the sound in question be completely unreliable and yet be true. And similarly the other sounds may have all been true, in which case the sound in question is completely reliable and yet may be false, since the set of ascriptors in terms of which reliability of a given ascriptor is assessed does not include the ascriptor in question. Truth (as " $T$ ") and reliability are therefore independent concepts. And this in spite of the fact that evidence as to the reliability of a sign may strengthen the belief that a sign is true, or vice versa.

This sharp separation of 'truth' and 'reliability' helps to clarify certain problems connected with the term 'probability.' Carnap ${ }^{F}$ has shown that this term is sometimes synonymous with 'degree of confirmation' and sometimes with 'relative frequency'; it is also sometimes synonymous with 'degree of belief.' Our analysis substantiates in semiotical terms his distinctions. For 'probability' in the sense of degree of confirmation is in our terms the degree of evidence that an ascriptor is true, while probability as developed by the frequency theory gives the degree of evidence that an ascriptor is reliable. And since the evidence in question is different (in one case that a given ascriptor denotes, in the other case that ascriptors similar to the given ascriptor denote) the two concepts of probability differ in spite of their common relation to the concept of degree of evidence. No discussion is required to show that probability considered as degree of belief is still a third, and less closely related, concept.

We have not attempted to state how the terms 'truth,' 'reliability,' 'belief,' and 'knowledge' are currently employed nor how in general they should be employed. Our task has rather been to make certain distinctions that are important for semiotic. So we have introduced the 
above terms in a limited way only; we have made them independent terms since it is feit that semiotic can then best avoid the confusions which now abound in talking about signs. In the interest of clarity we must not lump together such diverse matters as truth, reliability, and belief that signs are true or reliable, or the evidence that signs are believed or are true or are reliable. The distinctions are the important thing, regardless of what terms are used to signify the distinctions. ${ }^{\text {, }}$

\section{The Limits of Signification and Knowledge}

Since a sign can denote without its interpreter knowing whether or not it denotes, and since an interpreter may know that a sign denotes without himself directly encountering its denotatum, it is evident that signification and knowledge are not limited to that portion of the world which acts as a direct stimulus to an interpreter's behavior. What is distant in time and space can be signified and known as well as the directly encountered world, and no narrow limits can be set to such signification and knowledge. Through signs the individual directs his behavior with reference to things and situations which he may never have encountered and never can encounter, and yet the evidence which gives the ultimate control of knowledge must always be found in situations in which he himself behaves.

A distinction is to be made between the locus of signifying, the locus signified, and the locus of confirmation. A sign-process always involves an organism which at a given time and place interprets something. But what is signified by the sign may be signified as at a time and place other than that of the occurrence of the sign, and the confirmation or disconfirmation may be at a still other time and place. So whether a sign denotes or does not denote depends on the locus signified and not on the locus of confirmation. The bell which is a sign to a dog sounds at a certain moment and is heard by a dog at a certain place, but it signifies food to be found at a later time in a pan at another place, that is, the dog is prepared to act in a certain way to objects with a different temporal and spatial location than that of the environment in which the bell is heard. This process may be very complex. The statement 'John expected Bill to come,' uttered by a given individual at a given 
time, signifies that John expected Bill to come at a time later than a certain unspecified time itself earlier than the time of signifying. Signs, then, may denote objects at temporal and spatial locations different from the time and place of their own occurrence.

It may at first seem that the preceding account of signs is incompatible with the signifying of past occurrences. For if a sign is a kind of preparatory-stimulus, it might appear that signifying always has a future reference; how, it may be asked, can an animal be disposed to respond to something which has already occurred? The answer lies in the fact that while the behavior prepared by a sign is always later than the sign itself, the behavior prepared may be to things which no longer exist or to an object or situation as having characteristics due to objects or situations which once existed but which no more exist. $X$ says some afternoon 'It rained this morning.' These signs designate a certain occurrence at a time previous to the time of signifying. This occurrence cannot be a stimulus-object to $X$. But $X$ is prepared to act to his present environment as having certain characteristics due to the earlier occurrence of rain. Any evidence as to the truth of the statement relevant to $X$ 's knowledge must be available to $X$ himself: he may observe puddles of water, note the condition of the ground, use his memory or notes he made in the morning, consult the newspaper, ask other persons. But whatever the evidence employed it is evidence that something occurred at a certain time earlier than the time of signifying. Hence the importance of distinguishing between the locus of signifying, the locus signified, and the locus of confirmation that something occurred as signified at the time and place signified.

These distinctions are necessary not merely with respect to signifying the past, but also for signifying future objects and spatially distant objects. Statements about the distant in time and space may be known to be true though it is impossible for their interpreters to be at these times and places. It is even possible to obtain evidence to confirm statements about the future prior to the occurrences which would act as denotata for such statements.

Evidence is either indirect or direct, depending upon whether what serves as evidence is or is not a sign. Direct evidence that an ascriptor denotes is obtained by reacting to the region identified; if the responses 
which the ascriptor prepared take place, the ascriptor denotes (is a T-ascriptor). If the ascriptor is designative, the evidence is that objects have the characteristics which support the response prepared; if the ascriptor is appraisive, the evidence is that the interpreter of the sign accords to the object or objects the preferential behavior to which he was disposed by the sign; if the ascriptor is prescriptive, the evidence is that the interpreter of the sign does act to the object itself in the specific manner he was disposed to act because of the sign. In all of these cases ascriptors which signify that objects have certain properties are tested by the behavior of the interpreters of the ascriptors when in the presence of the objects in question. Behavior to objects is here the evidence, and such behavior is not a sign. The evidence is then said to be direct.

But in most situations this is not so: the evidence is "indirect." With respect to the truth of statements about the past or distant objects or future events the evidence is always indirect. Taking the experience of memory or the testimony of other persons as evidence for the truth of a statement about the past are instances of such indirect evidence. Here the evidence that a sign denotes is not given by direct response to a denotatum of the sign but by interpreting another event as a sign that the sign in question denotes (that is, has a denotatum at the time and place signified).

Since such indirect evidence is itself a sign, it has a certain degree of reliability which may be known in terms of the degree to which evidence of the kind in question has proved reliable in other instances. There are various ways, for instance, in which the reliability of memory or the testimony of others can be tested (a collection of objects can be shown and the memory of a person and the reliability of his reports tested by noting what he can recall after the collection is removed from view and the accuracy of his reports made while viewing the collection). Signs of varying degrees of reliability can thus be employed as evidence as to the truth or reliability of other signs for which direct evidence cannot be obtained. In this way statements about the past, the future, and the spatially distant can be known to be true without the interpreter of the statements being able to observe the events signified by the statements. The knowledge of an individual can in this way extend far beyond the limits of his immediate environment, and this knowledge 
can be effective in the control of his behavior in his immediate environment.

To what extent, however, can an individual signify and know what he cannot directly encounter ${ }^{\mathrm{H}}$ The limit of signifying is as wide as the ascriptors which individuals can produce by combining signs. Even if it be held that the signification of every component sign in an ascriptor is acquired by contact with something which is a denotatum of the sign in question, it does not follow that every denotatum of a sign must be an actual stimulus to the interpreters of the sign, for this would deny sign generality. And when signs are combined into ascriptors new significations appear the denotata of which may never have been encountered ('the earth a million years ago') or which can never be encountered ('things too small to be sensed').

The limit of knowledge is as wide as the range of evidence which may be secured that ascriptors are true. Since this evidence may be of any degree of indirectness, the limit of knowledge is potentially as wide as the limit of signifying, though practically there are always ascriptors which are understood and even believed and yet which are not known to any significant degree. As long as there is any evidence that a given ascriptor denotes, the lower rungs of the ladder of knowledge have been reached, and the possibility can never be excluded that some evidence can be obtained which bears upon the truth or reliability of any ascriptor which we can produce. It follows that the limits of signifying and knowledge are only practical limits and do not arise from any inherent defect in the nature of signs.

\section{The Control of Non-Designative Ascriptors}

While on our account any kind of ascriptor can be a T-ascriptor or not a $T$-ascriptor (and so true or false in a wide sense of these terms), this fact does not abolish the differences between kinds of ascriptors or differences in the way they are controlled. Since the designative ascriptor signifies characteristics of objects, it is accepted or modified or rejected primarily in terms of observation of identified objects or in terms of observation of reliable evidence that identified objects 
have the characteristics that are designated. But since in appraisive and prescriptive ascriptors what is signified about objects is not their characteristics but how they are to be favored or how they are to be dealt with, such ascriptors are mainly modified or rejected in terms of their adequacy with respect to the needs of their interpreters. But since such adequacy can be signified by designative ascriptors, knowledge of the adequacy or inadequacy of non-designative ascriptors becomes one important factor in their control. Since needs themselves change, and new ways of behaving are discovered to meet old and new needs, non-designative ascriptors adequate at one time are inadequate at another. Such ascriptors are therefore more liable to change than designative ascriptors, and their change is not solely a matter of knowledge.

Suppose, for instance, that a certain food object has been signified as "good," and that the status signified corresponds in fact to the preferential place given to that object in the food behavior of the interpreter of the appraisive ascriptor. If now the physiological needs of the interpreter undergo change, he will normally exhibit differences in his preference for various food objects and will not himself signify the given food object as good nor accept the appraisal of another person who signifies this object as good. The signification of the term 'good' need not change in this process, but the appraisive ascriptor which ascribes goodness to the given object will not be produced by the interpreter and will lose its effectiveness when encountered. And this change can take place without the interpreter designating or knowing the change or its causes. This occurs frequently in the different kinds of poetry which an individual will write or read at various times: love poetry will make its appeal under certain circumstances and the poetry of the Waste Land will carry another day. As needs differ appraisals tend to differ.

The change in the production and effectiveness of appraisive ascriptors may, however, vary with knowledge even when needs remain fairly constant. It may be possible to convince oneself or others by the use of confirmed designators that a certain object which has been given a preferential status in behavior does not in fact satisfy certain 
needs of the person, and this knowledge may cause a change in behavior to this object and influence the production and effectiveness of signs appraisively signifying this object.

The situation is similar with respect to prescriptive and formative ascriptors, and hardly needs elaboration. A command will normally lose its persuasiveness if the need which the commanded action satisfies is not present or if the individual knows that the action does not in fact meet an existing need. A formative ascriptor which explicates the relation between terms in a language will normally lose its correctness if a change of needs requires the development of a language with a different structure or if it is known that the ascriptor is not in accord with the structure of an accepted language. In such cases the use of nondesignative signs varies with needs and with knowledge as to the adequacy with which they meet needs. This control is an empirical control, dependent upon the adequacy of the signs as they operate in behavior and upon the knowledge of such adequacy.

The understanding of this situation throws light upon the question of the relation of designators to other signs. There is a widespread tendency in the literature of semiotic to take designation as the basic notion and to hope that all other modes of signifying can be avoided even if they cannot be "reduced" to the designative mode. We can now see the reasons for and against this view. Designation is basic in the sense that the other modes of signifying frequently involve designation, while designation can occur without signs in the other modes. Add to this the fact that knowledge about the adequacy of non-designative signs is a powerful factor in their control, and that signs in any mode of signifying can be used to realize any of the purposes for which signs are employed, and the case for the preeminence of designators seems strong.

This emphasis is particularly congenial at this time when, due to basic shifts in the value structure of society and vast increases in the techniques by which needs may be satisfied, the traditional appraisors and prescriptors have become increasingly inadequate. Since scientific language is pre-eminently designative in mode and informative in use, the tendency is strong to place reliance upon scientific discourse and to avoid, or even derogate, the other types of discourse. In so far 
as such "scientism" serves the purpose of obtaining more adequate appraisors, prescriptors, and formators it is inevitable and salutary; in so far as it discourages the use of non-designative signs it is theoretically questionable and culturally dangerous. For organic processes necessitate giving preferential statuses to some objects rather than others, the selection of some courses of action rather than others, and the choice of certain linguistic structures among various possible ones. And since all phases of behavior reflect themselves in sign-behavior and need signs for their effective operation, behavior is stultified when it attempts to dispense with signs other than designators (and hence with types of discourse other than the scientific).

It is true that in principle the valuative and incitive uses of signs can be performed by signs in some one mode of signifying, ${ }^{\mathrm{I}}$ and that if a choice has to be made designative signs are the most likely candidates. Instead of using appraisors one would simply designate the characteristics of objects and their relation to needs; instead of using prescriptors one would simply designate the way in which certain modes of action upon certain objects satisfy or thwart various needs. It is in fact possible to go very far in this direction, leaving it to each interpreter to determine on the basis of such information what he will prefer and how he will act. There is even a certain moral gain in this procedure, since interpreters are not coerced in their preferences or actions.

But the attempt to replace all non-designative signs by designators breaks down before the empirical fact that designators are not in general as adequate for valuative and incitive purposes as are signs in the other modes of signifying. Not merely are they cumbersome and circuitous in these respects; they are often highly inefficient. For the accurate description of a situation does not necessarily lead to a common preference by various interpreters or to common actions which are needed and which signs are used to effect. Hence the basic importance of signs which in themselves have appraisive and prescriptive signification common to members of a group-and it is such signification which designators, by their very nature, do not have. Common valuations and actions may of course be set up by the use of designators, but it is more direct and efficient to use for this purpose signs which in themselves appraise and prescribe. The role of des- 
ignative ascriptors in the control of non-designative ascriptors remains; this role is not to supplant these other ascriptors but to give us knowledge about them relevant to their improved usage.

\section{Communication}

The term 'communication,' when widely used, covers any instance of the establishment of a commonage, that is, the making common of some property to a number of things. In this sense a radiator "communicates" its heat to surrounding bodies, and whatever medium serves this process of making common is a means of communication (the air, a road, a telegraph system, a language). For our purposes 'communication' will be limited to the use of signs to establish a commonage of signification; the establishment of a commonage other than that of signification-whether by signs or other means-will be called communization. A person who is angry may be the occasion for another person becoming angry, and signs may or may not be the means of establishing the commonage: this is an instance of communization. Or a person who signifies anger may by the use of signs cause another person to signify anger without necessarily becoming angry: this is a case of communication. The user of signs who effects communication is the communicator and the organism in which the sign-process is aroused by the signs of the communicator is the communicatee. The communicatee may be the same organism which is the communicator, as when one writes a note to oneself to be read at a later time. The signs used are the means of communication and the signification made common by these means in the content of communication.

Communication, in this restricted sense, is not a new use of signs but a special case of the incitive use, namely, the use of signs to incite in the communicatee sign-behavior similar to that of the communicator (that is, of the same family of sign-behavior). Communication is designative, appraisive, prescriptive, or formative according to the mode of signifying of the content of communication. The establishment of communication may in some cases be the sole purpose for which signs are used. But normally communication is desired in order that some further purpose be realized, whether this purpose be informa- 
tive, valuative, incitive, or systemic. All of these purposes involve communication as a state in their realization. The goal intended may or may not be that of communization. An angry person may communicate his anger at something in order to cause the communicatee to be angry at the same thing (and so to communize anger), but equally well a person who is angry may communicate his anger in order to cause the communicatee to fear or respect him. Communica. tion may be used to make people different as well as to make them similar, and communization or differentiation between persons may be established by other means than communication. Since sign-behavior is itself a phase of behavior, to control the sign-behavior of other persons is a powerful means of controlling their total behavior, but the control intended may be for any purpose, moral or immoral, divisive or unificative, differentiative or communizative.

The adequacy of communication must be distinguished from the question as to whether the further purposes which motivate the communication are realized. A person communicating anger in order to make another person angry may succeed in the communication (the establishment of a commonage of signification) without producing the intended anger. The communicatee has, it is true, a tendency to respond in a certain way when a certain sign-process has been incited in him. But whether this tendency will take overt form depends upon many factors other than the signs themselves. It depends upon the needs of the communicatee: an animal that is not hungry will not in general seek food even if the existence of food at a given place is accurately communicated to him. It depends upon certain reactions of the communicatee to the signs, reactions which often involve additional self-produced signs: an estimation, perhaps, of the reliability of the signs by which he is offered information. It depends on the attitude of the communicatee to the communicator: suspicion of the communicator will reduce the readiness to act overtly in the way signified as desirable by the communicator. The fact that communication is established does not at all insure in any automatic way that the purposes for which the communication is made will be realized.

Since communication is the establishment of an at least temporary similarity between the interpretants of communicator and communi- 
catee, the problem of communication itself is how to attain this similarity. The solution is given in part by the existence and extension of language. For language signs have a common core of signification to members of a given linguistic community. Such interpersonality is slowly acquired, but when acquired is then transmitted to the young of the community. The child learns by various social rewards and punishments that in the community of English speaking persons the term 'good' is, among other things, a positive appraisor, that 'tree' denotes certain objects and not others, that 'Come here!' signifies that a certain action is demanded, that parentheses show what signs are to be combined. Hence such language signs are fairly reliable devices by which one member of the community can call out in another a desired process of signification, and so effect communication. Since communication is involved in all of the primary uses of signs, its effectuation and improvement is of basic importance.

The difficulties arise in the fact that interpersonality of language signs is seldom completely achieved, and that even where a common core of signification is obtained, the signs may have to different individuals of the community different additional significations. ${ }^{J}$ The term 'good' is at times an appraisor and at times a designator, so that a person intending to communicate an appraisive signification may by the use of this sign actually produce in another individual a designative sign-process, and thus fail in communication. Or a term which has a common designative core may differ appraisively to two individuals, so that the total communication intended by a person may fail even though a partial communication is effected. In this case the communicator may attempt, by the further use of signs, to improve the communication at the point where it is defective. Since this can be done only if these further signs themselves have (or can be given) some common signification, it is evident that the existence of language signs is an important agency for communication. Such signs insure communication over the area of their common signification, and are valuable in correcting the defects of communication which arise from the fact that signs-including language signs-differ in some degree in their signification for different individuals.

Basic improvement in communication is therefore dependent upon 
the attainment of a body of signs (designators, appraisors, prescriptors, and formators) which have a high degree of similarity of signification to different members of a community, and upon the attainment of skill in using these signs to make clear the particular signification which a given individual intends to communicate in a specific situation.

\section{Belief, Denotation, and Adequacy}

In this chapter we have insisted upon distinguishing the questions whether a sign denotes, whether it is believed by someone to denote, whether it is known to denote, and whether its production is useful to the realization of a certain goal. We must now bring the discussion to a focus by considering the interrelation of these topics.

The general importance of T-ascriptors ("true sentences") lies in the fact that expectations based upon such signs will not be disappointed. If a sign not merely signifies food at a certain place but denotes (that is, is a T-designative ascriptor) then any behavior based on belief that there is food at the given place will not, in that respect at least, be frustrated. And this holds also with respect to the reliability of a sign, though to a less degree, for while if an animal acts in accord with the reliability of a sign it may at times be disappointed in its expectations of what will be encountered, the number of such disappointments will be less in proportion to the reliability of the sign.

Even at the level of animal behavior organisms tend to follow the lead of more reliable signs. This follows from the basic laws of learning, according to which habits are strengthened or weakened depending upon whether connections of stimuli are or are not accompanied by a reinforcing state of affairs which reduces the need of the animal. At the human level, where signs are themselves signified, great effort is spent to find out what signs are true and what reliability signs have, and the resulting knowledge about signs becomes an additional factor determining which signs are to be followed in behavior. Such knowledge is gained not only about the denotation of signs, but also about the adequacy with which certain signs fulfill certain purposes. And this knowledge, as well as knowledge about the truth and reliability of signs, tends to influence the adequacy of signs. For if an 
individual knows that a given sign is unreliable this knowledge will affect the extent to which he is subject to influence by a second individual who uses the sign to exert such influence.

There is then a strong tendency for organisms to modify their beliefs in accordance with the reliability of signs. But there are also strong counter-tendencies at work, stemming in part from the persistency of habits and in part from the deliberate recognition that false and unreliable signs may for some purposes be more adequate than true and reliable signs. Certain beliefs may have become of central importance in the organization of a personality, and prove highly resistant to change even when the signs expressing the belief are shown to be highly unreliable. In this case the individual may in various ways disbelieve the evidence upon which unreliability of the sign is claimed by someone else, a fact which shows how difficult it is for signs to retain their adequacy when the signs are unreliable or are believed to be reliable.

And in case an individual is using signs to control the behavior of someone else, signs which he believes or knows to be false or unreliable may still be more adequate for the accomplishment of his purposes than signs which he believes or knows to be true and reliable. But even here the general dependency of the adequacy of signs upon their reliability is evident in the fact that the producer of signs in such a case must use signs which the person he is influencing takes to be reliable and must carefully guard against the communication of his own belief in their unreliability.

Beliefs, then, in spite of their persistency, do tend to vary with the reliability of signs. And the adequacy of signs, while not identical with their denotation, is in general dependent upon whether the signs do denote. The facts make intelligible the crucial role which the scientific search for true and reliable signs plays in the sign-behavior of man, and at the same time show why scientifically certified signs are not the only kinds of signs which human beings employ. This leads us to the question of the differentiation of scientific discourse from other types of discourse and its relation to such uses of language as occur in art, religion, mathematics, and philosophy. 


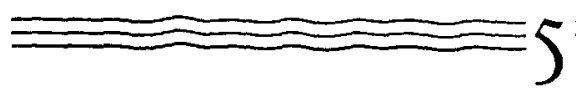

\section{TYPES OF DISCOURSE}

\section{Classificatory Basis}

7 HE language of everyday speech is an amazingly complicated sign complex which contains signs in all of the modes of signifying and which ministers to a vast variety of purposes. In the course of time various specializations of this common language have appeared in order that certain purposes may be more adequately met. Such specializations of language will be called types of discourse. Books, for example, are classified as scientific, mathematical, poetic, religious, and the like; and within these larger classifications subdivisions and overlappings occur almost without limit. The task of the present chapter is to provide a basis for a classification of the various specializations of language which will take account of current distinctions of laymen and semioticians and yet be less ambiguous than these distinctions prove to be. ${ }^{\mathrm{A}}$

A classification of types of discourse made in terms of basic semiotical principles can take several possible directions. Distinctions might be sought solely in terms of the modes of signifying, or solely in terms of the uses of sign complexes, or in terms of mode and use jointly. ${ }^{\mathbf{B}}$ Let us look at these three possibilities in turn. An attempt might be made to classify any given sign complex in terms of the relative proportion in which ascriptors in the various modes of signifying occur in it. Thus, sign complexes in which designators predominate may differ from each other in the relative frequency in which appraisors, prescriptors, and formators appear. And similar variations 
are possible in case the discourse is dominated by some other mode of signifying. In this way there would be six possible types of designative discourse, six possible types of appraisive discourse, etc., and, as a total, twenty-four possible basic types of discourse. The attempt would then be made to correlate as far as possible current distinctions among the specializations of language with these twentyfour possibilities. Discourse predominantly designative, secondarily formative, thirdly prescriptive, and lastly appraisive, might seem to correlate with scientific discourse; discourse in which the signs are in order prescriptors, designators, appraisors, and formators might be correlated with religious discourse. And so on.

This approach has certain merits. It would make possible a quantitative study of the various types of discourse (using ascriptors, for instance, as the unit of measurement), and it accords with the observation that a given type of discourse (say religious discourse) can be used for various purposes-since a type of discourse is not on this approach defined in terms of the category of use. These are strong points, and any acceptable classification will want as far as possible to incorporate them. But there are certain disadvantages to a classification based on the category of modes of signifying alone. The major one stems from the fact that it does not easily accommodate itself to current distinctions among the types of discourse. Suppose for example that a certain article begins by prescribing a course of action, but that the bulk of the article consists of factual statements which are used to support the desirability of the action prescribed. In this case a merely quantitative count of the kinds of ascriptors would lead to a classifcation of the discourse as predominantly designative while common usage would certainly tend to regard it as a prescriptive type of discourse. $^{\mathbf{o}}$

This situation, among others, has led some persons to seek a classification of types of discourse solely in terms of usage. Then the previously mentioned article would be called a form of incitive discourse, since it was used to incite a certain action, regardless of the kinds of signs used. In general, religious discourse would be defined in terms of a certain (probably incitive) usage, scientific discourse would be defined in terms of another (presumably informative) usage, and so 
on. This approach is theoretically valid and agrees in certain respects with prevalent distinctions. But it sacrifices the quantitative possibilities of the classification in terms of modes of signifying, since a given type of discourse could use signs in any mode of signifying. And the tendency, say, to call any piece of writing a poem if it is used in a certain way jars with the common terminology which this approach seeks to placate.

A third possibility, which retains some of the merits and avoids some of the difficulties of both the preceding approaches, may be called the mode-use classification. Here a type of discourse is characterized both in terms of a dominant mode of signifying and a primary usage. Pairing the four main modes with the four main usages gives sixteen major types of discourse (designative-informative, prescriptiveincitive, formative-systemic, etc.). Then the problem is to investigate the relation of these sixteen possibilities to the specializations of language currently employed and distinguished. For example, scientific discourse may perhaps be an instance of designative-informative discourse, and religious discourse an instance of prescriptive-incitive discourse; it is then necessary to find additional distinctive features of scientific and religious discourse which will distinguish them from other instances of the same type (since, for instance, not all designativeinformative discourse is scientific nor all prescriptive-incitive discourse religious). As a first approximation the following illustrations of the sixteen major types of discourse are proposed:

Examples of the Major Types of Discourse

\begin{tabular}{l|l|l|l|l}
\hline Mode & Informative & Valuative & \multicolumn{1}{|c|}{ Incitive } & \multicolumn{1}{|c}{ Systemic } \\
\hline Designative & Scientific & Fictive & Legal & Cosmological \\
\hline Appraisive & Mythical & Poetic & Moral & Critical \\
\hline Prescriptive & Technological & Political & Religious & Propagandistic \\
\hline Formative & $\begin{array}{l}\text { Logico-mathe } \\
\text { matical }\end{array}$ & Rhetorical & Grammatical & Metaphysical \\
\hline
\end{tabular}


The first twelve of these types of discourse will be discussed and illustrated in the present chapter; the last four in the following chapter. The language of philosophy is left for separate treatment.

It must be realized that the illustrations are tentative and indicate merely the problem and a direction of solution; we are not proposing a definition of, say, 'religion' or 'religious discourse,' but illustrating a type of discourse-in this case prescriptive-incitive discourse-by religious literature. It is also necessary to stress the fact that since we are dealing with specializations of language, the classification of a given piece of literature as being a certain type of discourse is in terms of what it normally signifies to members of a certain language community and how it is normally used by members of this community.

\section{Scientific Discourse}

Scientific discourse illustrates the most specialized form of designativeinformative discourse. In it the designative mode of signifying is freed to the maximum degree from the other modes and developed in ways which most adequately perform the task of conveying true information about what has been, does, or will exist. In this respect, it merely elaborates and refines such statements as occur in common speech. As science advances, its statements become more purely designative, more general, better confirmed, and better systematized. Scientific discourse is therefore made up of those statements which constitute the best knowledge of a given time, that is, those statements for which the evidence is highest that the statements are true. Science is especially concerned with the search for reliable signs. The goal to which science moves is a systematized body of true statements about everything which has occurred or will occur. But since at each moment the selection of statements for admission to science is dependent upon evidence that the statements are true, the selection will vary as new evidence is obtained, and so the scientific discourse of one time may differ greatly from the scientific discourse of another.

It follows from this description that any statement which cannot be confirmed or disconfirmed has no place in scientific discourse. For science seeks knowledge, and knowledge, as the term is here used, 
requires evidence. If therefore it is impossible to obtain evidence (direct or indirect) that a statement is true or false, the statement in question falls outside of science. In practice the decision on this point is often difficult and tolerance is wise, but the criterion of confirmability remains in the nature of the case a minimal criterion of scientific statements. Those statements for which at a given time the evidence is low are called hypotheses, while the best confirmed statements are called laws and form the basis for the systematization of science.

Science moves toward its goal by first restricting itself to the designative element in signifying. Then it attempts to gain greater precision by the development of co-ordinate systems which permit greater refinement in temporal and spatial identification, by the substitution of numerical signs for the indefinite 'some,' 'few,' 'many' and 'most' of everyday discourse, and by the introduction of carefully defined designators which signify characteristics of the environment neglected or unnoticed at simpler levels of observation. Finally, it develops an elaborate machinery of instruments, experiments, and training in order to improve the technique for selecting those statements which denote (and so are true) from those which do not denote. Such pruned, precise, and confirmed statements, systematically organized, constitute the body of scientific knowledge at any time. Any discourse composed of such statements is scientific discourse. ${ }^{\mathrm{D}}$

The concern of scientific discourse to attain statements which are known to be true arises from the importance of obtaining reliable information about the environment in which behavior operates or which it will effect. In formulating such statements it makes available for any act knowledge of the environment which the act will encounter in the environment designated. It accordingly serves as a basis for accurately determining predictions as to what conditions behavior will have to deal with. The scientific statement does not approve or disapprove of the conditions it states, nor of any particular act under these conditions; the data it presents are relevant to any act directed upon its denotata. Scientific statements as to how phosphorus reacts to air and water are appraisively and prescriptively neutral; they simply state that substances with such and such characteristics have such and such further characteristics under such and such conditions; the information con- 
veyed may be used to build incendiary bombs, to extinguish them, to avoid any contact with them whatsoever, or to use phosphorus in a variety of ways. In abstracting from any particular goal, science gives information relevant to a variety of goals.

A point previously made bears repetition in this context. While truth is not defined in terms of accuracy of prediction, it follows from the nature of truth that predictions based on a true statement will prove to be accurate, that is, that what is expected as a result of the information conveyed by a true statement will occur as expected. For if the statement is true that dry phosphorus bursts into flame in air, then 'inflammable' denotes what 'dry-phosphorus-in-air' denotes; therefore if one encounters dry phosphorus in air, one can expect correctly that it will burst into flame. If a statement is true, expectations based upon it will be satisfied, and the confirmation of such expectations, though it does not define the signification of 'truth,' is the basis for knowledge that the statement is true.

A person may be interested in scientific statements for their own sake (interested in collecting them as a person may be interested in collecting butterflies); a person may have knowledge and the increase of knowledge as his goal. Nevertheless, it follows from the very nature of knowledge that expectations based on statements known to be true will not be disappointed, and genetically this significance of scientific knowledge has played a large part in the development of science. It is certainly no accident that scientists at a given time are markedly concerned with getting knowledge relevant to the problems of that time; while science does not appraise or command a particular act, the knowledge that it seeks is significant knowledge, that is, information relevant to the accomplishment of various acts. No scientist has set himself the task of measuring the distances between the top of the Eiffel Tower and the corners of the headstones in all the cemeteries of Paris.

\section{Fictive Discourse}

Fiction in its various forms may be taken as an instance of designativevaluative discourse. In fictive discourse some sequence of happenings is designated, but whether the events happened as narrated is not, as 
in scientific discourse, the central issue; fiction explores an imagined universe rather than delineating the actual universe. And in fiction there is likewise a minimizing of appraisal or prescription; though the fictitious characters (if the fiction be of persons) may themselves appraise and prescribe, their appraisals and prescriptions occur within the work as additional fictional elements rather than as characteristics of the dominant mode of signifying of the work itself. A novel about slums designates individuals in a certain imagined environment, shows their interactions with each other and their environment, and presents their ideas and attitudes in such interactions, but it does not as a novel claim fidelity to an actual situation, nor appraise slum conditions, nor tell us how we ought to act with regard to slums. And though our actions and attitudes may be influenced by the work, and by such indications as we can gather concerning the position of the author himself on these matters, these considerations do not determine our classification of the work as fiction.

This does not mean that fiction is unconcerned with questions of value. Although its mode of discourse is primarily designative rather than appraisive, its primary purpose is valuative rather than informative. Fiction abounds with heroes and villains even when it does not label them as such. It aims to induce preferential attitudes to what it designates (and often to the discourse itself) even though it does not itself specifically appraise itself or the environment imagined. The telling of the tale is to be approved and the events narrated are to be found significant; if neither result is attained, the work has failed of its purpose. Fiction then not merely presents an imagined series of incidents, but it signifies these in such a way that the interpreter is constantly led to prefer certain of the signified events and characters, though the valuations induced may differ widely from interpreter to interpreter (partly because the work itself does not, as fiction, appraisively signify). The writer and the reader of fiction understand that what is signified is fictively and not scientifically signified, and both are interested in fiction because of its relation to their preferential behavior. The world of fiction is an imagined and fictive world important because of some interest (some preferential behavior) with respect to what is signified. 
This view does not deny that fiction may contain scientifically true statements nor even that belief in the truth of certain statements may be a factor in determining the adequacy of fiction. The interest in the description of a utopia may be dependent upon the belief that the imagined society is not only physically possible (compatible with known empirical laws) but that it is probable (that events are moving in a direction such that the imagined society will one day be actual). Insofar as the events depicted are incompatible with existing knowledge, fictive discourse becomes more and more fantastic and in time becomes absurd. This suggests that fictive discourse remains related, though often tenuously, to the actual world and actual behavior, supplying as it were an imaginative exploration of possible environments congenial to such behavior. Utopias are projected by persons interested in a world different from the one they inhabit, but a world different only in certain respects, namely, in those respects which would provide a more congenial environment for their actual needs. In general the reader of fiction reads those works which designate the kind of environment in which he is interested and which present this environment in a way which supports his own valuations.

The importance of fictive discourse resides in the fact that it provides a presentation through signs of significant imagined environments. It permits the interpreter not only to delight in the way the story is told and indulge symbolically his actual preferences, but to get material to test, to reconstruct, and to form his preferences. The liberating quality of fictive discourse lies in the exploration which it permits of how life might be lived in various ways in various environments.

\section{Legal Discourse}

The language of law furnishes an example of designative-incitive discourse. Legal discourse designates the punishments which an organized community empowers itself to employ if certain actions are or are not performed, and its aim is to cause individuals to perform or not to perform the actions in question. Legal discourse as such does not appraise these actions nor prescribe them; it does not say that it is good to act legally or that one should so act. It merely designates the 
steps the community says that it is prepared to take if certain actions are performed or not performed. It does not even say that the community will actually take the steps signified (i.e., it makes no predictions of a scientific sort about society), though if the community persistently fails to take such steps legal discourse becomes progressively inadequate with respect to its incitive aim.

While legal discourse is primarily designative, its aim makes its designative status different from that of scientific or fictive discourse. It designates the ways a given community has committed itself to act if the members of that community behave in certain ways. A given body of laws designates the practices a given community has committed itself to control by the use of force. The laws which are so laid down, and which the discourse states, designate commitments, and are neither fictive nor scientific. Not fictive, because a commitment is something actual and not imaginative; not scientific because the law states how a community is committed to act under certain circumstances and not how it will act (a law which is not administered is no less a law because of that fact). Even such a sentence as 'John Doe is guilty of larceny' is a sentence in legal discourse only when competent legal authorities have decided that John Doe is guilty of larceny. It is true that the decision may, though it need not, rest on evidence which science would accept as trustworthy, but the fact that the sentence remains legally binding when decided by delegated legal authorities until overruled by another legal decision, even though new evidence has established its falsity, indicates that its legal status is not determined by its truth or falsity as a statement. ${ }^{E}$ Similarly, appraisals of the law or prescriptions with respect to law belong not to legal discourse itself but to some other form of discourse (moral, critical, technological, etc.), while statements about legal processes or legal discourse fall in the science of law.

The basic point that has been made can be remade in a way that comes to the same thing. It is sometimes said that a constitution, whether written or unwritten, is the ultimate test of when a sentence is a sentence of legal discourse, since the constitution determines who are the authorities competent to make judicial decisions and thus give to sentences a legal status. But since a constitution in this wide sense is simply the organization of the society in its way of determining which 
activities it shall force to be performed or prevented, or the symbolic expression of this organization and its commitments, the determination of the legal status of a sentence by appeal to a constitution simply restates the criterion incorporated in the preceding account of legal discourse.

Legal discourse aims to inhibit or encourage the individual in the performance of those acts which some social group aims to control by the application of its organized power. Its sphere may be very wide, determining what religious activities and what moral solutions are permissible; or in some societies, such as our own, it may leave great freedom to religious and moral practices. Through its legal discourse a social group aims to secure for itself a body of common and dependable practices to protect and advance its own organization; and the individual who "lays down the law" to himself is again following within the social commonwealth of his own personality the pattern of legal procedure exemplified in social groups.

\section{Cosmological Discourse}

It is difficult to identify among the commonly recognized types of discourse one that clearly illustrates designative-systemic discourse, that is, discourse which while designative is used for the purpose of organizing sign-behavior provoked by designators: The difficulties stem from a number of sources: signs are normally used systemically in order to fulfill some other use, so that it is especially difficult to distinguish in the case of designative-systemic discourse (indeed, in the case of all systemic uses of signs) the discourse itself from the further uses to which it is put; it is also difficult to distinguish the type of discourse in question from the unification of knowledge which would be part of scientific discourse, or from the organization of sign-produced behavior which we propose later to distinguish as metaphysical discourse. Nevertheless the theoretical possibility of designative discourse aiming to organize designative signification remains, and it would be surprising if the specializations of language showed no exemplification of this possibility. 
The best illustration of what is meant by cosmological discourse is found in the world views of the philosophers. ${ }^{\mathrm{F}}$ We are told by them that the universe is one or many, that it is material or spiritual, that it is composed of substances or events, that it is purposive or mechanistic, that it is hostile or friendly to human values.

These pictures of the world are stated in a primarily designative language; they have the "tone" of science and often its content; they seem to inform the interpreter what the world "really is." Hence the easy confusion of them with science, and hence also the frequent attempts to distinguish them from science by the claim of some "superempirical" truth found by some special method (such as the "dialectical" method). The present suggestion is that cosmological discourse is different from science in that it aims to organize the main designators accepted by a given individual at a given time, and so may include fictive and legal designators as well as poorly confirmed statements and current scientific knowledge. This hypothesis explains the frequent feeling that cosmological language is charged with "speculative" statements and with fictive and often even legal elements, as in the doctrines of "natural law." That such discourse is not in its entirety scientific is brought out by the fact that alternative world views are framed in the face of the same scientific evidence and persist in spite of the change and accumulation of such evidence; hence they are not in toto composed of highly confirmed statements as are genuinely scientific theories. This suggests that they include more than scientific statements, and the present hypothesis is that this "more" is found in the totality of statements which a given thinker believes to be true. The cosmologies organize basic beliefs about the nature of the world; while they may include science, they extend beyond it.

So conceived, cosmological discourse is a distinctive type of discourse, designative-systemic in character. Though it embraces more than science it is not to be rejected as "meaningless" or "emotive" or "unimportant." Its significance lies in the fact that men need an organization of the statements they believe to be true as one phase of their total orientation. What organization is most adequate will depend both on the knowledge of the time and on the purposes dominant in a given 
community or in a given individual. Insofar as science is dominant in a culture, cosmological discourse will include science and will change to some degree as science changes. But scientific statements are never the whole of the designative component of a given individual and information about the actual world is never the sole purpose operating in behavior. So the adequacy of a cosmology varies with cultures and individuals as well as with changes in science. Only if 'truth' be confused with 'adequacy' is it necessary to defend cosmological discourse by claiming that it is science or that it gives superscientific knowledge. Such defenses are to be understood more as devices to make such discourse adequate than as true explanations of its nature.

\section{Mythical Discourse}

We now turn to the four types of discourse which are predominantly appraisive in their mode of signifying. The first of these is appraisiveinformative discourse. The kind of discourse represented in mythology may serve as an example, though the term 'mythical,' like most such terms in current usage, has a wide range of signification, often overlapping with the terms 'fictive' and 'cosmological,' or negatively appraising statements which are not scientifically accepted (and thus often coinciding with certain of the depreciative uses of 'metaphysical'). The term 'mythical' as here used covers a type of discourse which is neither fictive (since its aim is primarily informative) nor scientific or cosmological (since its ascriptors are primarily appraisive). In mythical discourse the signs are appraisive of certain actions and aim to give information as to the preferential status accorded by some person or group to these actions.

In one of the stories of the Buddha's previous incarnations he is said to have been a rabbit, and to have offered himself to a hungry monk for food, first shaking himself before he plunged into the fire in order that no insects in his hair might be killed. The story as told employs many statements to be sure (since most signifying involves designation), but it is told in such a way as to inform the reader of what Buddhists esteem in a language strongly appraisive of the self-forgetful- 
ness of the rabbit and its kindness to other living things. And neither of these features of the myth requires the interpreter to believe literally in the doctrine of reincarnation nor in the historical actuality of the events signified, though of course he may hold such beliefs and hold the discourse to be designatively-informative of historical occurrences, that is, he may not regard the discourse as mythical. This situation makes clear that an interpreter of any discourse may be mistaken as to what type of discourse it is, and that a set of signs which in a given community is normally one type of discourse may for a specific individual not operate as that type of discourse. How far a given individual or group of individuals distinguish mythical and, say, scientific discourse, and how interpersonal a given set of signs is to various individuals, are empirical problems, and do not affect, but rather presuppose, such semiotical distinctions as we have made. The relevant distinction here is between appraisive-informative and other types of discourse; we have merely said that myths, in one sense of the term now current, serve to illustrate such discourse.

Such an interpretation of mythical discourse permits us to explain the divergent interpretations of myths given by contemporary writers. Thus Ernst Cassirer stresses the cognitive character of the myth, Bronislaw Malinowski stresses the place of the myth in social organization, and others interpret the myth esthetically. ${ }^{6}$ That the myth is informative in purpose and so is distinguishable from fiction makes intelligible Cassirer's emphasis; while the fact that the myth may be effectively used for purposes of social organization (since it appraises and informs the individual of the group's standards of behavior) gives leverage to Malinowski's treatment. That the myth may incorporate poetry or form the subject for poetry explains how it may be confused with poetic discourse, the type of discourse to which we next turn.

The general significance of mythical discourse lies in the fact that it informs the interpreter in a vivid manner of the modes of action approved and disapproved by some group (or in the extreme case, by some individual). It thus makes available to the interpreter information concerning an important body of appraisals which he may utilize in his behavior, whether by way of agreement or disagreement. 


\section{Poetic Discourse}

Poetry may serve as an example of discourse which is primarily appraisive-valuative. Poetic discourse, so conceived, signifies by signs which are appraisive in mode and its primary aim is to cause the interpreter to accord to what is signified the preferential place in his behavior signified by the appraisors. Whitman writes:

I believe in the flesh and the appetites,

Seeing, hearing, feeling are miracles, and each part and tag of me is a miracle.

Divine am I inside and out, and I make holy whatever I touch or am touched from.

The scent of these arm-pits aroma finer than prayer,

This head more than churches, bibles, and all the creeds.

Flesh and the appetites are here manifestly approved individually and in their multiplicity; they are specifically signified as finer than conventional religious institutions and literature: to them is attributed the values historically attributed to religion. Designation of course occurs, as it does in most signifying, but it occurs as a way of isolating what the appraisors signify. That arm-pits have a scent, that the human personality is complex, are, to be sure, statements and are subject to confirmation, but the appraisive superstructure is evident in such terms as 'miracle,' 'holy,' 'aroma': how test by observation that feeling is a miracle? or that Whitman made the beer glass out of which he drank holy? or that the aroma of arm-pits is finer than a prayer? These ascriptors are appraisals and subject to the special kinds of controls which characterize appraisals. What the poem does is to signify a preferential place to the body and its desires as over against certain products of man's religious activities.

The illustration also brings out, though not in an especially vivid way, the reasons why poetical discourse is so frequently described (by Shelley, for instance) as metaphorical. A sign is metaphorical if in a particular instance of its occurrence it is used to denote an object which it does not literally denote in virtue of its signification, but which has some of the properties which its genuine denotata have. To call an automobile 
a beetle, or to call a picture of a man a man is to use 'beetle' and 'man' metaphorically. Since an automobile is not literally a beetle, to call it a beetle forces the interpreter to attend with special care to the automobile in order to determine in what sense the automobile is like (and unlike) a beetle. So if the significances of objects are to be signified, the metaphor in which the metaphorical term is highly appraisive is a handy instrument, since the interpreter is then forced to consider the significance of the object in order to find the common element which is signified. To call feeling a "miracle" or objects touched by a certain person "holy" or the scent of arm-pits "aroma" is a powerful way of causing the interpreter to signify certain objects as having the importance ordinarily given to the literal denotata of the terms in question. This explains the high place which metaphors have in poetic discourse without equating poetry and the metaphorical use of language-for not all metaphorical terms are appraisive.

It is perhaps more doubtful that the aim of poetic discourse is primarily valuative. It may appear that appraisive signifying, especially when metaphorical in character, is sufficient, and that there is no further need to claim that the poet aims to excite valuations. There are undoubtedly senses in which this contention is true. A person can, of course, know that a certain discourse is poetic in the sense that it operates as such for certain other persons without it operating as poetic discourse for him. But there is still the question of the criterion by which it is called poetic discourse, and I believe this involves the aim of inducing valuative attitudes. This does not mean that such attitudes are maintained over any given length of time or even that the interpreter will accord in his overt behavior the preferential status to objects signified in the poem. But the fact that preferential behavior does not take overt form does not mean that it is not present. The matter is complicated by the further fact that the valuative attitudes the discourse aims to induce may be primarily to the discourse itself rather than to what is designated. Undoubtedly in the development of poetry this emphasis occurs, and to the poetic craftsman the interest in the way signs are employed becomes the predominant interest-so much so that such persons may wish to limit poetic discourse to appraisive discourse which aims to induce approval of the discourse itself. Even if this 
restriction is made, poetic discourse remains appraisive-valuative discourse. But since protests frequently arise when the virtuosity of the poet becomes so limited that he writes solely for other poets to approve his writing, the wisdom of this restriction is doubtful.

The great significance of poetic discourse lies in the vivid and direct way that it records and sustains achieved valuations, and explores and strengthens novel valuations. In poetry the object signified is turned, as it were, before our eyes with symbolic fingers, and as we look at the object described and exemplified by the poet, we come in varying degrees and for a longer or shorter time to take the valuative perspective of the poet in terms of which the object signified has the apprehended significance. The interpreter will naturally seek and prefer those poems most in accord with his own valuative attitudes, but even in them his own impulses will be somewhat modified and somewhat differently organized; and insofar as other poems can gain his attention, he will vicariously try out new valuations for familiar objects and familiar valuations on unfamiliar objects. In this process the valuative attitudes evoked by the poems will be intensified, modified, reorganized in various ways. In taking the valuative attitudes of others, one reacts back on one's old valuative attitudes, and the system of valuations and appraisals stands the test or is accordingly changed. Poetry then not merely records what in fact men have found significant, but plays a dynamic role in the development and integration of valuative attitudes and explicit evaluations. Poetry at its best is a symbolic antenna of behavior at the immediate frontier of its valuational creativity. How far what is true of poetic discourse can be extended to apply to art generally is a question to which we will return, but the relevance of the question shows the significance of semiotic for the general problems of esthetics. ${ }^{\text {I }}$

\section{Moral Discourse}

Appraisive-incitive discourse may be illustrated by the language of morality. For discourse that appraises actions as favorable (or unfavorable) from the standpoint of some group, and aims to incite (or inhibit) these actions, is certainly close to what is commonly recognized 
as moral in character. It is true that the term 'moral' is often more widely used to apply to discourse which we shall classify as critical or technological or religious, but the combined appraisive and incitive character (with group welfare as the focus) seems to be the central and differentiating element. ' Not all 'oughts' or all prescriptions of techniques to be followed are called moral discourse, but few persons would hesitate to ascribe this term to language which appraises actions in terms of group welfare. Further, the close interrelation of the moral and the social is an almost universal theme in the writings of ethicists.

From this point of view moral discourse is linked with the sphere of social behavior. We have seen that groups of organisms may have goals in whose realization individual organisms play certain specialized roles. Further, these individuals may, in ways suggested by George Mead, come to symbolize the role of other individuals in social behavior and the needs of the group, and to appraise their own behavior or the behavior of other individuals with respect to the needs of others or the needs of the group. In terms of the perspective of social behavior the individual in this way gains a basis for appraisals in which he can even approve or disapprove of certain of his own preferences and actions. Appraisals made of oneself or others in terms of what is conducive to group welfare, when incitive in aim, constitute moral discourse. The term 'ought,' so common in moral discourse, is (when a moral 'ought') evidence of the dual appraisive and incitive character of moral discourse, for 'ought' signifies some action that is positively appraised, and is used in contexts which make clear that the user of the sign is endeavoring to incite the action in question.

The appraisals of moral discourse are very commonly the appraisals of actions customarily made by members of the group in question. Indeed, a moral appraisal which runs counter to such approved actions will in general be disapproved of by members of the group. But the fact that even these persons will generally call the appraisal (and the person who makes it) "immoral," while occasional individuals will even approve it, shows that the appraisal still falls within the sphere of moral discourse. Diversity in moral appraisals is made intelligible by the fact that the members of a group may be mistaken both with respect to the needs of the grcup and to the actions which serve, or best serve, its 
needs. So it is possible for there to be moral appraisals (appraisals made from the standpoint of group behavior) which differ from the customary moral appraisals of members of the group. Socrates' appraisals, in being condemned as immoral by his society or in being defended as of a superior morality by his friends, are in either case recognized as being moral discourse.

Moral discourse is of importance in the social control which it exercises over the conduct of individuals. To the extent that it is effective it causes individuals to act in the light of the effect of their actions upon other individuals of the social group to which they belong. To the degree that moral appraisals are built upon reliable knowledge of group needs and ways of meeting these needs, moral discourse is an important agency in advancing the consummation of group interests and the interests of individuals in a group. While moral discourse is frequently conservative, it is not inherently so, and when vital and adequate it not only accommodates itself to changes in social behavior and technological advances, but serves to facilitate the adjustment of a group to these changes.

Moral discourse has close relations to legal, technological, and religious discourse. Yet in its combined appraisive and incitive character it is distinct from them, and distinct also from the critical and scientific discourse which may be directed upon it. Not all criticism is morally oriented and a science of moral discourse is not itself couched in the language of morality.

\section{Critical Discourse}

What is often called "criticism" (or "evaluation") serves to illustrate appraisive-systemic discourse. A person asks his friend to criticize a manuscript. What he expects is an appraisal of the manuscript as a whole which is defended by the systematic organization of specific appraisals. We will call discourse critical in a slightly narrower sense than is usual: namely, when it is systematically appraisive of appraisals. So used, critical discourse has the same relation to the appraisive mode of signifying as cosmological discourse has to the designative mode. 
In critical discourse some body of appraisors is organized into a more complex system of signs itself appraisive in mode of signifying; in this way there arises a grounded appraisal of appraisals.

The criticism of a poem illustrates the situation. The critic of Whitman's lines quoted a few pages back will appraise them as good or bad and ground this appraisal in terms of an analysis of the appraisals in the poem itself. He may attempt to limit the sense in which the body and its desires are praiseworthy, question whether it is possible to compare valuatively such diverse things as scents of arm-pits and prayers, attempt to show inconsistencies in specific appraisals in the poem, disparage the words and style used by Whitman. All sorts of considerations may be employed by the critic to ground his own appraisals: he may question the statemental foundation of the poem, he may make statements about the effects of such a poem, he may compare it to other similar poems which he or others approve, he may call to his support moral, religious, metaphysical, logical, grammatical doctrines. But the designative features serve as grounds for his own appraisals and for their systematization. His own discourse as critic remains ultimately appraisive and his immediate aim is the systematization of appraisals to support some more comprehensive appraisal. Criticism may, of course, be undertaken for a host of other further reasons (informative, valuative, and incitive of various kinds) but the mark of critical discourse itself is its systemically appraisive character.

Varieties of critical discourse may be distinguished in terms of the kind of appraisors dealt with. Just as poetic criticism deals with poetry, so does moral criticism deal with the appraisors of moral discourse. Moral criticism is often represented in works of ethics; the characteristic ethical treatises (such as those of Aristotle, Spinoza, Kant, Mill) are not primarily designative and informative (i.e., are not primarily scientific studies of morality and moral discourse) but are grounded defenses of some body of moral appraisals. The same is true of theology as a criticism of the appraisals of religion; such criticism (say that of Aquinas) is a defense of a certain set of religious values within a specific religious tradition and is not to be confused with a science of religion. Ethics and theology, as forms of critical discourse, are to be distinguished 
both from moral and religious discourse, and from a science of morality and a science of religion.

Since critical discourse is appraisive in nature, its adequacy is that appropriate to such discourse; it is not, for instance, subject simply to the type of confirmation of scientific discourse. As we have seen, the critic may in the course of his evaluation attempt to ground his appraisals on scientific knowledge, but this is only a means toward his own concluding appraisal. As Abraham Kaplan has argued,' a critical appraisal in the end can be defended only by a continuation of the process of criticism itself. The adequacy of a scientific statement is tested by finding further data which increase or decrease its confirmation; the adequacy of criticism is tested by additional criticism. The situation is complicated by the fact that criticism may be carried on for a great variety of secondary purposes (informative, valuative, and incitive), and in this case further questions of adequacy arise with respect to the way it accomplishes these purposes. Nevertheless, there remains the primary purpose of critical discourse (the grounding of appraisals by a systematic process of appraising), and here the way in which a critical appraisal stands up under further criticism is the test of its adequacy. For there is no ultimate evaluation of preferences except in terms of preferential behavior.

Critical discourse emerges as a distinctive type of discourse. It allows its interpreters to organize their appraisals into more complex appraisals, and is thus one of the ways in which behavior attains organization at the appraisive stage of its development. Criticism may, but need not, start with specific appraisals which are then simply defended in the critical analysis; the critical reaction may at first be mixed in character, hesitant and uncertain, and the process of criticism serve to disentangle the confusion and organize the response into a unified whole which issues in a positive appraisal. The initial appraisals and preferences may be changed in the process. As a way of attaining appraisals based on careful scrutiny of appraisals, criticism helps to advance the act beyond its initial impulsive and indeterminate stages, and to give it determinateness and organization. Since criticism may be directed to any appraisals, it is relevant to the advancement of any act whatsoever. 


\section{Technological Discourse}

Discourse which prescribes actions for the purpose of informing interpreters how to attain certain goals is an instance of prescriptive discourse, and in particular of prescriptive-informative discourse; it is not unfairly called technological discourse. For such discourse aims to give information concerning the techniques for attaining specific ends, whatever they may be. It is "how to" discourse: discourse informing one how to rivet, how to play the flute, how to cook a duck, how to speak Spanish. Since the goals may be anything whatever, there is technological discourse relevant to science, to morality, to religion, to mathematics, etc. Such technological discourse neither appraises the goal for which it is relevant nor aims to incite the actions it prescribes for reaching a goal; a manual on flute playing does not extol the significance of the flute nor tell the person that he ought to acquire flute-playing techniques: it merely tells how to play the flute. And the case is similar for technological treatises in engineering, medicine, agriculture, and the like. A goal is taken for granted; the treatises tell how to attain it.

Since techniques do or do not attain the goal for which they are instrumental, and do so with greater or lesser efficiency, the adequacy of technological discourse is closely connected with knowledge about the efficacy of techniques. Manuel Andrade used to illustrate this by a story of the rain-making practices of certain American Indians. In collecting accounts of such practices he found one shaman who omitted a series of actions prescribed by other shamans, namely, the lighting of fires at night which were to be put out at the stroke of dawn. In inquiring into the reasons for this omission he was told by the shaman that he didn't "believe" in doing this, since one morning when a certain fire was not put out "the rain came anyway"-a result which was repeated when he again deliberately left a fire burning. Here we see how technological discourse changes as knowledge of its efficacy changes, a point that could be equally well illustrated in changes in medical practice.

What is often called "magic" is the persistence of techniques when there is evidence that the practices do not in fact influence the attainment 
of the goal, especially when these practices are symbolic in nature. The savage who, in instructing the young in canoe making, prescribes that a feather should be waved over the canoe at a certain moment in its construction so that its speed may be increased, is talking "magically" in that he is advocating a practice which we believe adds nothing to the speed of the finished canoe. And yet even here caution is needed; for the practice may, in the culture in question, give to those who use the canoe a greater confidence in the boat, and by increasing their confidence increase their efforts, so that a greater speed does in fact result. In this wider context the symbolic action may be technically relevant and the technological discourse which prescribes it be adequate. Such complications are not to be forgotten in assessing the adequacy of technological discourse for moral and religious ends.

Scientific knowledge acts as a constant source of control for existing techniques and is often generative of new techniques, yet technological discourse is not scientific discourse and is not controlled by it alone. To say that it "should" be is to speak prescriptively, and is only one prescription among others. Factually the most we can say is that technological discourse does normally vary with increasing knowledge of the reliability of the techniques prescribed. In a culture in which science plays a prominent role, science will undoubtedly exercise increasing control over the accepted technological discourse of that culture. But much of such discourse is highly resistant to scientific control and cannot be simply condemned on that basis alone if we are genuinely concerned with an analysis of the way signs operate in behavior. Indeed, it is certain that many of our practices for which we invoke the sanction of science-including some techniques in contemporary science itself-will be regarded by later generations as magical. This is a special instance of the general point that a person may be mistaken as to the type of discourse which he is producing or interpreting.

Technological discourse provides a way in which individuals may acquire information as to current techniques employed in attempts to reach various goals. Since this information is important if behavior is to move toward its consummation, technological discourse is and will remain an important specialization of language. 


\section{Political Discourse}

The prescription of actions with respect to the institutional organization of a society as a whole, with the aim of calling out approval of such organization, is characteristic of political ideologies. The corresponding "political discourse" seems to illustrate discourse which is prescriptive-valuative. The Declaration of Independence may be taken as an example; Rousseau's Social Contract or the Communist Manifesto would be even clearer illustrations.

The Declaration prescribes an organization of society in which all men are to be treated equally with respect to their "inalienable rights," rights which government is to preserve and respect. The whole document is couched in terms which aim to cause the reader to approve such a society. It brings to the support of the approval it aims to produce current moral and religious beliefs, as in the assertion that all men are created equal; and in its appeal to naturally inalienable rights it makes use of current metaphysical doctrines as to natural law. In these ways it aims to induce approval of the form of social organization whose erection it demands. It is not describing an existing society but prescribing the organization of a new society dedicated to securing life, liberty, and happiness. Its prescriptions are primarily, though not exclusively, valuative in aim.

That even such doctrines as the equality of men may be prescriptive rather than designative in character has often been recognized. The defenders of the equality of men need not regard it as a scientific doctrine. It may well be that all men are the same in certain respects and differ from all other beings in these respects, but if the doctrine in question is meant primarily as a true description of human nature it would be necessary to detail also the enormous differences between individuals. The words 'all men are equal' are admittedly designative and not prescriptive; they do not say 'Treat all men as equal.' But taken in their context they certainly are used to awaken approval for an attitude which treats all men equally in certain respects and for certain purposes, and the document as a whole is prescriptive of a form of social organization dedicated to implementing this attitude which it aims to 
induce. A later age may accept the document in this sense without accepting the grounds given for approving of the attitude invoked and the resulting scheme of social organization. Indeed, the failure to give a frankly prescriptive formulation of the equality of man may now weaken its present-day effectiveness.

The adequacy of such political discourse is determined by the extent to which it is able to call out approval for the form of society which it prescribes. Such adequacy, like that of all prescriptive discourse, will depend upon the convincingness of its designative base and upon its relevancy to the social problems of the group to which it is addressed. As knowledge changes and as social goals change, the political discourse of an earlier time tends to lose its effectiveness. Such documents then become objects of critical scrutiny, aimed either at buttressing up the effectiveness of the document or weakening it. In this way there arises a clash between those defending the existing social organization and those proposing a new form of social organization. Political discourse, in common with most types of discourse, is both an agency for social conservatism and for social reconstruction. It is a tool through which social behavior in its widest reaches aims to induce approval of certain institutional forms for the realization of its widest social goals. Its adequacy is to be measured in terms of its effectiveness for advancing the purposes for which it is used.

In the nature of the case political discourse has close relations to other types of discourse, and is difficult to isolate with precision. ${ }^{\mathrm{K}}$ Any particular instance of such discourse may contain features of scientific, fictive, moral, legal, technological, or religious discourse. But insofar as a unique core is identifiable it seems to lie in an attempt to prescribe a general course of action to support the form of social organization for which the discourse aims to induce approval.

\section{Religious Discourse}

Religious language, as prescribing the mode of behavior which is to be given precedence over all other behavior and as aiming to incite such behavior in its interpreters, is a striking exemplification of discourse that is primarily prescriptive-incitive. Religious discourse lays down the pat- 
tern of behavior which is to be made dominant in the total orientation of the personality and in terms of which all other behavior is to be assessed. $^{\mathrm{L}}$ In giving positive approval to one kind of personality rather than to others, it involves appraisors which signify the ultimate commitments (the supreme valuata) of the religion in question, but since it signifies this personality as something to be attained, its mode of signifying is prescriptive. And since its aim is to cause persons to become personalities of the kind prescribed, its aim is not merely informative or valuative but incitive. The languages of various religions differ with respect to the form of behavior (the ideal of personality) for which dominance is prescribed. In some (Christianity, for instance) moral behavior is assigned a very high place, and in such cases religious discourse and moral discourse may be very much alike; in others moral behavior is regarded as but a stage on the path to individual salvation (Buddhism) or as something which religious behavior may deliberately transgress (Dionysianism). The religious frame of reference may or may not coincide with other frames of reference, and may tolerantly include or intolerantly exclude other kinds of behavior.

In the religious literature of early Christianity, such as is represented by the Gospels, the individual is told to be a person dominated by the attitude and practices of Christian love: he or she is to restrain the impulses which make for lust, conquest, possessiveness, and to stand before God and man as a tender and sympathetic lover stands before his beloved. In early Buddhism the attitude and practices prescribed are not those of socialized and sympathetic love but those which seek and lead to a state (nirvana) in which all craving for existence and nonexistence alike has been overcome by an individual who depends on no one but himself for salvation. In Dionysian literature the self seeks release from intellectual and social restraints through an orgiastic freeing of its most elemental impulses. The commitments as to the finally preferred form of behavior (and hence the kind of personality taken as ideal) differ as these religions differ, and their literature prescribes and incites these ultimate commitments.

Around every religion there grows in time a body of critical discourse (a theology) which aims to defend systematically the way of life approved by the religion. Such discourse makes use of contemporary 
science and metaphysics in order to convince its interpreters that the way of life which the religion prescribes is in accord with cosmic and human nature. A religion will also seek esthetic embodiment for the ideals to which it gives allegiance, and will build up a technological literature giving in detail the techniques which the individual is to apply to realize the accepted personality goal. But neither the theology nor the poetry nor the technology are primary religious discourse.

The adequacy of religious discourse itself depends upon whether or not it appeals to given individuals in a given cultural milieu as a way in which their lives can be satisfactorily focused and directed. When this is not the case, new prophets appear to proclaim a mode of life which they have found significant, and if other individuals find the new way to be significant for themselves, a new religion and religious literature arises which will seek its esthetic embodiment, its appropriate techniques, and its critical defense. The complicated human self has need of some focal attitude to give it orientation, and the significance of religion lies in its attempt to meet this need. Since there is no evidence of a diminution of this need, it can safely be expected that religious discourse will continue to exist regardless of whatever development science undergoes, though it may take forms strikingly different from those embodied in the religious literature of the past.

\section{Propagandistic Discourse}

There is no very suitable term in common usage to name discourse which is prescriptive in character and systemic in aim, that is, discourse which organizes prescriptors and yet is itself prescriptive. We shall in this situation somewhat arbitrarily use the term 'propagandistic discourse' for what we have in mind. Such discourse is analogous to cosmological and critical discourse, but unlike them it is concerned with the organization of prescriptors through the use of signs themselves prescriptive. It too may be and is used for various other purposes (valuative, incitive, informative) but has as its primary use the systemic one. It buttresses prescriptions by relating them to other prescriptions. ${ }^{M}$

Examples abound in newspaper editorials, in advertising, and in the pages of the Congressional Record. A given newspaper, for instance, 
is against social security measures; in its editorial columns it argues that such measures weaken the initiative of those they protect; the prescription that we should protect the initiative of our citizens is used to buttress the prescription that we should oppose the measures for social security. Or the advertiser attempts to strengthen his claim that we should buy his product by relating it to real or reputed scientific testsand of course "we should be scientific." Or, again, the speaker before Congress who opposes the regulation of working hours of women thunders out that one's labor is one's property-and that because of the Constitution we must not deprive a person of his property without due process of law. In all these cases a certain connection of prescriptions is presented in discourse which itself is prescriptive.

As C. L. Stevenson has pointed out, such arguments make frequent use of what he calls "persuasive definitions": put in our terms, the speaker changes the denotation of certain common terms while continuing to use the existing appraisive and prescriptive features of their signification. The defender of the status quo will call anyone a "Communist" who proposes social changes as long as the term carries the power of prescribing repressive measures to anyone so denoted. Or a term like 'free' will, in certain democratic societies, be extended to any existing practices that a person wishes to perpetuate-since in such societies the protection and extension of freedom is prescribed morally and approved politically. Such a use of terms is really a prescriptive metaphor since it extends the denotation of signs in terms of a similarity of prescriptions with respect to the old and the new denotata.

Propagandistic discourse is somewhat difficult to isolate. Since prescriptors so often depend on appraisors, propagandistic discourse makes constant use of the language of poetry, morality, and criticism. And since the prescriptors which are being defended may be technological, political, or religious, propagandistic discourse takes on different forms which are not easily distinguished from the corresponding types of prescriptive discourse. Finally, propagandistic discourse is itself used for various other secondary purposes, and so is easily confused with discourse which is primarily informative, valuative, and incitive. Nevertheless, semiotic shows the possibility of a type of discourse which is prescriptive and systemic, and when analyzed with care, what we have 
called propagandistic discourse may be taken to exemplify this possibility.

The significance of propagandistic discourse lies in the organization of prescriptions. This organization may be over a small range of prescriptions or may extend over the whole range of existing prescriptions. It gives to prescriptions the support of other prescriptions, and thus strengthens these prescriptions in the control they exercise over behavior. The adequacy of propagandistic discourse is determined by the adequacy with which it performs such organization of the prescriptive elements of sign-behavior. That this adequacy is dependent in part upon the adequacy of its component statements and appraisals illustrates the previously discussed relation of prescriptors to designators and appraisors.

\section{The Study of Types of Discourse}

Before turning to the varieties of formative discourse, it is well to pause a moment to gain clarity as to what has been done.

The first point to note is that no definitions of the common names for types of discourse have been proposed. As far as the formal structure of semiotic is concerned, we have merely shown that there are sixteen possible major kinds of discourse if four modes of signifying and four primary uses of signs are accepted. We have not stipulated that the term 'religious discourse' is by definition identical with one of these sixteen possibilities. Rather, we have said that certain kinds of sign complexes sometimes classified as religious literature seem to have characteristics which bring them under the label of prescriptive-incitive discourse. Whether this is so or not so is an empirical matter and not a matter of definition: it can only be answered by taking documents or utterances which are commonly accepted as religious and submitting them to careful study. Since such a term as 'religious' has in the common language many significations, it is to be expected that documents and utterances which some persons call religious will not under analysis prove to be prescriptive-incitive discourse in the sense here defined. And this of course holds for the other terms which have been employed, such as 'cosmological,' 'poetic,' 'moral,' and so on. 
In the light of this situation it seems unwise at the present stage of semiotic to attempt to lay down precise definitions within semiotic for such terms. Accordingly we have merely used certain kinds of documents and utterances as illustrations of the kinds of discourse which are distinguishable in terms of the present framework of semiotic. The next step would be to study these and other such examples in detail. Much material for such a study is already available in the investigations which have been made of the language of poetry, fiction, law, religion and the like. Much more material can be found by carefully controlled studies of the kinds of signs found in such specializations of language and of the behavioral contexts in which such signs operate. The work of Harold D. Lasswell and his group is an illustration of directions which can be followed. ${ }^{\mathrm{N}} \mathrm{He}$ and his co-workers have been especially concerned with "political" documents (political and in part propagandistic discourse in the sense of our analysis), and have attempted to apply quantitative methods in this study. This intensive work in a certain area of the field of discourse has many features applicable to other fields; the terminology of the present book may help supply operationally defined categories for the extension of such empirical studies. As they proceed, the body of empirical knowledge within descriptive semiotic will grow. Such knowledge may in turn indicate the desirability of changes within the framework of pure semiotic itself. And in time it may be feasible to set up definitions of religious, poetic, and other types of discourse instead of using everyday material simply as illustrative examples. As this is done a precise and comprehensive classification of types of discourse will be obtained.

Even at the present time it is believed that the analysis of the preceding sections has power. It avoids the crude separation of types of discourse into scientific and non-scientific, and it shows in a sympathetic manner the unique importance of a great variety of specialized forms of language. ${ }^{0}$ It also suggests the interrelations of these varieties, the ways in which some of them utilize and depend upon others. While it gives to each a function, it also serves to unmask the ways in which they may seem to be something other than what they are, and so to help avoid the maze of confusions surrounding the question of the numerous kinds of adequacy or inadequacy to which language is subject. Com- 
plex human behavior needs all of these types of discourse, but it is ill served when one of them claims for itself an adequacy and importance which it does not possess. Any actual document or speech may of course be composed of all kinds of signs and used for the fulfillment of all the main purposes we have distinguished. But clarity as to the major types of discourse is essential if language is to become the magnificent instrument of man's total life which its nature permits it to be. ${ }^{\mathrm{P}}$ 


\section{FORMATORS AND FORMATIVE DISCOURSE}

\section{The Problem of Formators}

TN CHAPTER III brief reference was made to a kind of sign called 1 formator, and it was there stated that we would return to the diffcult problems posed by such signs. Those problems must now be considered in some detail, for they are basic to the complex question of the nature of formative discourse and to the interpretations of mathematics, logic, rhetoric, grammar, and metaphysics. This chapter will deal with these topics, beginning with a discussion of the formative mode of signifying, passing on to illustrations of four main types of formative discourse, and then considering the status of logic, mathematics, rhetoric, grammar, and metaphysics in relation to formative discourse and to semiotic. The reader should be warned at the outset that we are dealing with the most debatable issues in the whole field of the science of signs. The treatment, in the nature of the case, will be explorative; considerable advance in semiotic will be necessary before the problems can be satisfactorily resolved. Our main purpose is to show that these topics can be approached within a behavioral semiotic, and to indicate in these terms a direction for their solution.

We have considered at some length the conditions which a stimulus has to meet to be an identifior, designator, appraisor, or prescriptor. Let us henceforth call all such signs lexicative signs, or lexicators. Then in terms of our previous discussion of the term 'formator' we 
may say that if a stimulus is not a lexicative sign, but nevertheless influences in a uniform way the total signification of the particular sign combinations in which it appears, the stimulus in question is a formator. This formulation, however, leaves open the questions whether formators are signs, whether and what they signify, why they occur, and what-if any-features of language are formators.

On no point do semioticians differ more than on the interpretation of such items as 'or' '?' and '( )'. Some semioticians regard them as auxiliary devices influencing the signification of the sign combinations in which they appear, but without signification of their own; some as signs signifying special "formal" or "logical" properties of situations; some as designators signifying other signs; some as signs expressive of the states of their users; some as prescriptors of action with respect to things or signs. ${ }^{A}$ In the face of such diversity it is reasonable to surmise that the differences are not factual but arise in part from the acceptance of different terminologies in which to talk about signs. Here the semiotician must be careful to take upon himself the advice he likes to give to others: Do not confuse terminological and factual problems!

The terminology which has been developed in preceding chapters is incomplete and does not settle the problem at issue. For it has left open the question as to whether or not we are to admit as signs things which do not fulfill the conditions of being preparatory-stimuli influencing the dispositions to response to something which itself is not at the moment of signifying a stimulus to behavior. If there are phenomena which are commonly regarded as signs but which do not fulfill these conditions, either we can say that they are not signs within this system of semiotic or we can enlarge the class of things admitted as signs within this system by laying down additional sets of conditions under which the term 'sign' denotes. The term 'signification' can then be extended to such signs or restricted to signs which fulfill the initial set of conditions (i.e., to lexicative signs). It is therefore possible to develop the present system of semiotic in such a way as to regard 'or' '?' '( )' as signs without signification in the sense previously defined. One would simply say that any stimulus, not a lexicative sign, which influences in a determinate way the signification of sign combinations 
in which it appears is a formative sign (or formator); it would not be necessary to attribute to the formator as such any signification, nor to give any definition of 'sign' in terms of which lexicative signs and formative signs are subvarieties. This procedure is not only compatible with our previous discussions but it is in agreement with the position of many contemporary logicians. It may be that this is all that is required to do justice to formators. The reader is free to adopt such a position if he wishes, and we are free to return to it if the alternative which we will propose proves under analysis to be unsatisfactory.

The alternative which we will endeavor to defend is to regard formators as signs in the previous sense of the term, that is, as preparatory-stimuli influencing the dispositions to respond to something which is not at the moment of signifying a stimulus to behavior. Under this interpretation formators will differ from lexicative signs in terms of their interpretants, that is, in terms of the particular kind of disposition to respond which they occasion. The behavioral problem is then to isolate the distinctive interpretant for formators, and correspondingly, the distinctive kind of signification which formators have.

The advantage of this approach, if it can be carried through, is the simplification and uniformity of the language of semiotic which it permits. For it would mean that at this point at least it has proved unnecessary to add a totally new set of conditions under which something is admitted to be a sign; formators become a subdivision of signs as the term has been previously used, a "variety" but not a new "species" of signs. So 'sign' would remain an unambiguous term; and a formator would signify in the sense that other signs signify.

Is the difference between these two alternatives factual or purely terminological? I think that it is in one sense factual. It is a fact that what are generally admitted to be languages include such devices as 'or' '?' '( )'. Therefore semiotic, if it is to include the entire study of languages, must deal with such devices. It is also a question of fact whether on the account of signs given up to this point such devices are denotata of the term 'sign,' and if so whether they can be regarded as lexicative signs (that is, whether the introduction of the term 'formator' is required for their analysis). But here the factual phase of the problem ends. If such devices are not signs in the previous sense of the 
term, then semiotic must be extended so that it is applicable to such devices. And this extension can be carried out in a number of ways.

So our problem is whether such items in a language as 'or' '?' and ' $(\cdot)$ ' are signs in the sense that lexicators are signs, and if so whether they are themselves lexicators or a variety of signs co-ordinate with identifiors, designators, appraisors, and prescriptors. We shall argue that such items are signs, but formators and not lexicators. If this claim cannot be defended then the alternatives are to regard such items as lexicative signs of one kind or another, or to extend the terminology of semiotic by laying down a totally new set of conditions under which something is admitted into the class of signs.

\section{The Nature of Formators}

Since the situation is clearly complex and easily engenders unwarranted verbalizations, let us keep in mind the behavioral facts which we aim to describe by the introduction of the term 'formator.' And so back to the dog! Suppose that $S_{1}, S_{2}, S_{3}$ are signals to the dog of food in three different places, so that the dog, when hungry, seeks food in the place signified by the stimulus presented to it. Now if a new stimulus, $S_{6}$, be combined always with two of these other stimuli (as in, say, $S_{1} S_{6} S_{2}$ ), and if the dog then, without preference, seeks food at one of the two places signified and at the other place if and only if food is not secured at the place first approached, then $S_{6}$ would be a stimulus which has much in common with the exclusive 'or' of English ('at least one but not both'). If the dog could be further trained so that the signs which appear with $S_{6}$ may be appraisors and prescriptors as well as designators, then $S_{6}$ and the exclusive 'or' would be behaviorally identical.

The introduction of the term 'formator' is intended solely to designate this and similar situations. The signification of the combination $S_{1} S_{6} S_{2}$ is different, as shown by behavior, from that of $S_{1}$ or $S_{2}$ alone, or from that of $S_{1}$ and $S_{2}$ together. 'Formator' simply gives a way of referring to the properties of such stimuli as $S_{6}$.

Is $S_{6}$ itself however a lexicative sign? That it does not lexicatively 
designate is made evident by the fact that it does not identify the location of food or characterize certain objects as food objects (this is done by $S_{1}, S_{2}$, and $S_{3}$, and by such sign combinations as $S_{1} S_{6} S_{2}$ ). Nor does it itself appraise one food location as preferential to another, nor prescribe any particular response-sequence for obtaining food. So it does not lexicatively signify with respect to things other than signs. Nor with respect to signs. $S_{6}$ does not signify characteristic of signs, nor appraise signs, nor prescribe responses to signs. It occurs only in sign combinations, ${ }^{B}$ but it no more signifies signs lexicatively than it signifies things other than signs.

The question then is legitimate whether formators are signs at all in the sense in which lexicators are signs. Let us look more closely at the way in which $S_{6}$ affects the dog's behavior in order to see if we can isolate a kind of interpretant distinctive of a formator. $S_{1}$ disposes the dog to seek food at a certain place, and $S_{2}$ to seek food at another place. What behavior is the dog disposed to because of $S_{6}$ when it is presented along with $S_{1}$ and $S_{2}$ ? It is a complex disposition to response in which the interpretants of $S_{1}$ and $S_{2}$ are related in a certain way: the dog is disposed to seek food at one place if food is not found at the other place, and not to seek food at one place if food is found at the other place. The disposition to relate the interpretants of other signs in this way is the distinctive interpretant of $S_{6}$, for $S_{6}$ establishes this disposition regardless of what other signs it accompanies. It is a second-order disposition (interpretant) since it is a disposition to relate other dispositions (interpretants) in a determinate way.

$S_{6}$ is then a sign in the previous sense of the term. For the essential feature of a sign lies in its giving rise to an interpretant, and this $S_{6}$ does. $S_{6}$ determines how the dog will respond to one of the signified places in virtue of what it encounters at the other; it therefore is a preparatory-stimulus influencing the response to a later stimulus. It is a preparatory-stimulus controlling behavior as do situations in which the dog has learned to seek food in one of two places when and only when it is not found in the other. If a situation of this sort be called a situation of alternatives, then $S_{\mathbf{v}}$ may be said to signify the property of alternativity. What has the property can only be determined by 
other signs, so that $S_{6}$ will occur only in sign combinations; it occasions a distinctive linkage of response-sequences within the behavior-families related to the accompanying signs in these sign combinations. And when $S_{\mathbf{6}}$ does occur in ascriptors these ascriptors will be true or false, reliable or unreliable, confirmed or disconfirmed as any other ascriptor.

$S_{6}$ then not merely conforms to our account of a formator, but proves to be a sign as that term has been used, and to signify in the same sense that lexicators signify. It differs from lexicators however in that it does not characterize, appraise, or prescribe with respect to a situation but signifies that the otherwise signified situation is a situation of alternatives. Such signifying is an instance of what we have called the formative mode of signifying, and the signification-in this case alternativity-is a formatum.

If we now generalize our analysis we obtain the following result: Formators are signs which dispose their interpreters to modify in determinate ways the dispositions to response occasioned by other signs in the sign combinations in which the formator appears. The conditions under which these dispositions to response can be actualized (that is, the conditions under which formators denote) are called formative properties or formata. Formators occur in the formative mode of signifying, that is, signify formata.

In terms of this result it might seem advisable to transform our previous set of conditions under which something was admitted to be a sign into a definition of 'sign.' For one of the main reasons for not giving a definition of 'sign' was to leave open the possibility of regarding formators as signs in case they did not fulfill the initial set of conditions. Nevertheless, I think it would be premature to take this step. Not only may there be hidden difficulties in the analysis of formators which has been given, but there may be other kinds of signs which our analysis has not uncovered and which we do not wish arbitrarily to exclude from semiotic. So it seems wise not to give a general definition of 'sign.' It is sufficient for the moment to have at least made plausible the position that formators are signs in the sense that lexicators are signs, and yet signs in a distinctive mode of signifying. 


\section{Kinds of Formators}

Formators are made necessary by the facts that the lexicators of a language are often general, may be combined with each other in a variety of ways in specific sign combinations, and may occur in sign combinations of various modes of signifying. Formators serve to influence in a particular sign combination the way in which certain component signs signify or the way in which the combination as a whole signifies. Three large classes of formators may be differentiated in terms of what kinds of influence the formators exert upon the interpretants of the signs with which they are combined. We shall make no attempt to give an exhaustive classification of formators, but merely show the basis upon which such a classification might be erected.

Determinors are formators which determine the range of possible denotation which a general sign has in the particular sign combination in which it appears with the formator (that is, signs which determine whether the interpretant of a general sign is to remain general or is to be restricted in the given sign combination). 'All' in 'all houses' signifies that no restriction is imposed in this particular instance on the houses signified, while 'one' in 'one house' signifies that the way houses are signified is in this instance singular. Numerical terms such as '2' and ' 10 ' determine exactly the range of denotata of some general sign which is signified in the sign combination in which they appear. 'Some' is less definite in the restrictions imposed, since it is compatible with the denotata signified being either singular or unrestricted; it excludes the possibility, signified by 'no,' that the range of denotata of some general sign which is signified in the sign combination in which it appears is null. ${ }^{\mathrm{C}}$ Behaviorally, determinors influence the range of denotata of general signs to which the interpreter of the sign combinations in which they appear is disposed to act. It may be pointed out that the general sign whose interpretant is influenced by the formator is determined in various ways, the simplest of which is physical adjacency of the sign-vehicles; such a device draws attention to the relevant general sign but it does not follow that the formator signifies this sign.

Connectors are formators which establish a particular connection 
between the interpretants of other signs in the given sign combination in which they appear. They may be intra-ascriptor connectors or interascriptor connectors, depending upon whether they connect the interpretants of the component signs of ascriptors or connect the interpretants of ascriptors into a compound interpretant. Commas, parentheses, "gender" devices, 'is' and 'is not' illustrate the former kind of connectors. Thus ' $(2+2) \times 4$ ' differs in signification from ' $2+$ $(2 \times 4)$ ' in virtue of the position of the parenthesis; in the first case what is established by the parenthesis is the summation of denotata, while in the second case what is established by the parenthesis is a product of denotata. Gender endings are in rare cases lexicative, as when they signify sexual characteristics, but in general they simply serve to connect the interpretants of certain signs with those of certain other signs in particular sign combinations, and have no lexicative signification: that the term 'table' in French requires certain endings in certain sign combinations shows nothing about the characteristics of tables. 'Is' and 'is not,' as formators, signify the formative properties of attribution and non-attribution; they determine that the interpretants of the signs with which they appear are connected in such a way that the interpreter is disposed to act toward certain identified objects as having or not having certain properties.

Inter-ascriptor connectors are illustrated by certain instances of 'and,' 'or,' 'implies.' 'And' may have a lexicative component, as when it designates togetherness ('Pierre and Yillah pulled the log', that is, pulled it together); it is a formator insofar as it merely signifies the formative property of being conjointly signified. ('Pierre pulled the $\log$ and Yillah pulled the log'; here it is not necessarily signified that they pulled the $\log$ at the same time). 'Or' in the exclusive sense has already been analyzed; in its wider sense it signifies non-exclusive alternativity (' $\mathrm{He}$ is a scientist or an artist'). 'Implies' is often a metalinguistic designator, signifying a relation of implication between ascriptors (' $x$ is red' implies ' $x$ is colored'); but in the sense frequent in current logic in which 'implies' signifies merely "either not the first or the second," it is an inter-ascriptor connector definable in terms of 'or' and 'is not' ('His coming will imply his willingness,' that is, 'Either he will not come or he is willing'). 
Another kind of inter-ascriptive connector is illustrated by the devices which establish subordination among the interpretants of ascriptors in a compound ascriptor. 'That red tree is beautiful' is a compound ascriptor which includes the ascriptor 'that tree is red'; the position of 'red' before 'tree,' together with the absence of 'is' as a connector of the two terms, subordinates the interpretant of this ascriptor within the total ascriptive signification; 'is,' then, is not merely an intra-ascriptive connector but is often an inter-ascriptive connector establishing the superordination of the interpretants of certain signs.

Modors are formators which establish the ascriptive mode of signifying of the sign combination in which they occur (that is, determine whether the total interpretant composed of the interpretants of the other signs in the given sign combination is to be the interpretant of a designative, appraisive, or prescriptive ascriptor). ' $\mathrm{He}$ is coming' may be spoken in such a way that the ascriptor is a statement, an appraisal, or a prescription--signifying merely that an event does occur, or signifying that his coming is good or bad, or signifying that his coming is inquired about or demanded. The intonations and speech melodies which effect these differentiations are modors. In writing, the corresponding modors are the punctuation devices '.' '!' '?' '!!,' though there is no sharp standardization here of appraisive and prescriptive devices. In both speech and in writing special devices other than intonation and melody may occur to indicate the differences ('Fine that-,', 'Is he-,' 'He must--,' etc.); often the absence of a special device is itself a modor establishing the mode of signifying as designative.

The isolation and classification of formators does not require that the signs which signify formatively may not also have a lexicative component, but merely that over and above any such component they have a distinctive non-lexicative character of the sort described. ${ }^{p}$ Formators often seem to be developed from previous lexicators (as 'is' comes from a word which signified "to grow") and seldom lose entirely their lexicative character. But it is essential to recognize that in their capacity of formators they are not lexicative, and that a language may contain, and perhaps at times does contain, signs which are only formative in their mode of signifying.

Formators, then, are distinctive kinds of signs. The difficulty in 
isolating their status has many causes, but is certainly due in part to the fact that most thinkers have, here as elsewhere, been content with selfobservation. They have looked at a sentence such as 'He will come or he will phone' and then tried to describe introspectively the role of 'or' in this combination. The variety of results shows the difficulties of such an approach. Only if we first clarify the behavioral distinctions in terms of which we classify lexicators are we in a position to decide whether such signs as 'all,' 'or,' and '?' are lexicative, and only if we study formators in terms of their effect on behavior are we able to isolate the distinctive character of such signs in relation to lexicators. The present account is undoubtedly defective in many ways, but it at least points a direction in which the confusions and disagreements in this field may in time be overcome.

\section{Formative Ascriptors}

Formative discourse, conceived in analogy to the differentiation of other types of discourse, is discourse composed primarily of formative ascriptors. How now may we identify a formative ascriptor? Two suggestions which present themselves at first glance prove not to be satisfactory. One might suppose that an ascriptor is formative if it contains a formator, but this is too wide a definition since then almost all ascriptors would be formative. Or one might try to distinguish formative ascriptors as those which contain only formators, but this proves too narrow a definition, since it would exclude such sentences as 'Tomorrow it will rain or not rain in Chicago.' ${ }^{\mathbf{E}}$ What is wanted is some way to set off formative ascriptors from lexicative ascriptors that will accord with the commonly recognized distinction of "logical" and "factual" sentences ("analytic" and "synthetic" sentences, "L-determinate" and "L-indeterminate" sentences). And while formators must somehow play the crucial role in making the intended distinction, it is neither their individual presence nor their exclusive presence which gives what is wanted.

The criterion proposed is as follows: A formative ascriptor is a compound ascriptor such that the denotation of one or more of the component ascriptors (called the antecedent ascriptors), is, because of the 
signification of the ascriptor, a sufficient condition for the denotation or lack of denotation of the remaining component ascriptor or ascriptors (called the consequent ascriptors), and hence for the denotation or lack of denotation of the compound ascriptor itself. Formative ascriptors which denote if their antecedent ascriptors denote will be called analytic; formative ascriptors which do not denote if their antecedent ascriptors denote will be called contradictory; ascriptors which are neither analytic nor contradictory are lexicative ascriptors, and are said to be synthetic.

The following examples, to be analyzed later, illustrate these three kinds of ascriptors. 'That black berry is black' is an analytic formative ascriptor since it is sufficient for 'that is a black berry' to denote for 'that is black' to denote, and hence for 'that black berry is black' to denote. 'That black berry is not black' is a contradictory formative ascriptor since it is sufficient for 'that is a black berry' to denote for 'that is not black' not to denote, and hence for 'that black berry is not black' not to denote. But 'that blackberry is black' is a lexicative (synthetic) ascriptor since 'that is a blackberry' may denote and yet 'that is black' may or may not denote (that is, blackberries are black at some stages of their growth and not black at others).

Before seeking the behavioral formulation of the distinctive status which formative ascriptors have among ascriptors, one objection to the above analysis may be noticed. It might be asked why we did not merely say that formative ascriptors are such that they are those ascriptors which are always true or false, regardless of factual considerations, and so regardless of denotation. The answer is that if we keep to our definition of 'truth' ('T-ascriptor') in terms of the denotation of ascriptors, then the truth of formative ascriptors must also be stated with respect to denotation. And we cannot evade this by saying that an analytic formative ascriptor is one which "by necessity" denotes, for denotation is not something which in terms of our previous terminology can ever be determined from a consideration of signification alone, that is, no ascriptor "necessarily" denotes as we have used 'denote.' Nor can we say that a contradictory formative ascriptor is one which "by necessity" does not denote, for no ascriptor denotes if its component signs do not denote-hence this proposal would give us no way of differentiating 
contradictory formative ascriptors from other ascriptors. Therefore the differentiation of formative from lexicative ascriptors is not made on the basis of omitting reference to denotation, but rather in terms of whether the denotation of the component antecedent ascriptor or ascriptors is or is not a sufficient condition for the denotation of a compound ascriptor. ${ }^{\mathrm{F}}$ 'All red things are colored' as analytic does not say that there are red things, but merely combines signs in such a way that if there are red things, they are colored, that is, such that being denoted by 'red' is a sufficient condition for being denoted by 'colored.'

\section{The Behavioral Basis of Formative Ascriptors}

We must now seek to explain behaviorally the distinctive status of formative ascriptors. Logicians have shown that many analytic formative ascriptors are of the pattern ' $S_{1}$ or not $S_{1}$,' and many contradictory formative ascriptors are of the pattern ' $S_{1}$ and not $S_{1}$.' So we will take such patterns as our examples. The distinctive element in such ascriptors is found in relating ascriptors of the same sign-family by the combination of formators 'or' and 'is not,' or 'and' and 'is not.' The 'or' in this case will be taken as the exclusive 'or' ('at least one but not both'). 'Is not' we have seen to be a connector establishing a relation of exclusion between interpretants in a sign combination, and so signifying nonattribution. Hence to our $\operatorname{dog}$, ' $S_{1}$ or not $S_{1}$ ' signifies that food is at place 1 or food is not at place 1. What does this involve in terms of his behavior?

We have already analyzed the dog's behavior when he is signaled food in one place or in some other place. The peculiarity of the present sign combination is that food is signaled to be or not to be at the same place at the same time. Certain of the component signs dispose him to seek food at the given place while others tend to negate his disposition to seek food at that place. But these interpretants are connected by the formator 'or' into a complex interpretant such that the dog is disposed to seek food at the place in question if he does not find food at that place, and is disposed not to seek food at the given place if he finds food at that place. All of which is undoubtedly rather hard on the $\operatorname{dog}$ ! And yet that this behavioral situation obtains cannot be denied 
if $S_{1}, S_{2}$, 'or' and 'not' are signs to him. For if an animal has an interpretant for ' $S_{1}$ or not $S_{1}$,' the interpretant in question is obtained when $S_{2}$ is substituted for $S_{1}$.

The uniqueness of this interpretant lies in the way in which the interpretants of the component signs are related. The formator 'or,' in this combination with 'not' and two sign-vehicles of the same signfamily, links two interpretants in such a way that in any given situation if one is prevented from issuing in overt form this fact alone permits the other to take overt form. Put in terms of expectation, the expectations of the animal are so linked that if in a given situation one is satisfied the other is thereby thwarted, and if one is thwarted the other is thereby satisfied; if the dog does not find food at the place identified then his expectation that he will not find food is confirmed, while if his expectation that he will not find food is thwarted his expectation that he will find food is confirmed.

Now this is the same thing as saying that an implicate relation has been established between the signs. It will be recalled that one sign (say, 'colored') is said to be an analytic implicate of another (say, 'red') if the conditions for the first sign to denote are the same or part of the conditions for the second sign to denote. In the present case the formator 'or' has established implicate relations between 'food at place 1 ' and 'food not at place 1,' that is, the denotation of 'food at place 1 ' is sufficient to insure the denotation of 'food not at place 1' and the denotation of 'food not at place 1 ' is sufficient to insure the denotation of 'food at place 1.' So not only is 'food at place 1 or food not at place 1' an analytic formator by our criterion, but its nature is found to involve an implicate relation between signs, and that in turn to be expressible in terms of a relation of interpretants such that if one is prevented from issuing in overt behavior that fact alone supplies the conditions for the other to issue in overt behavior. Interpretants in such a relation may be called analytic interpretants. All analytic formative ascriptors are believed to be of the same general nature as that revealed in our example. They presumably all involve signs which are analytic implicates, that is, they presumably all involve analytic interpretants.

The contradictory formative ascriptor ' $S_{1}$ and not $S_{1}$ ' is analogous. Only here contradictory interpretants are involved in that if one issues 
in overt behavior that fact prevents the other from issuing in overt behavior. Hence in that case there is a contradictory implicate relation between signs, the condition for the denoting by one sign being a sufficient condition for the other sign not to denote.

In this way we have attempted to carry out George Mead's hint that the relation of implication depends upon a relation of responses. ${ }^{1}$ And if our account is correct formative ascriptors always rest upon an implicate relation between their component antecedent and consequent ascriptors, and so find their behavioral basis in analytic or contradictory interpretants.

\section{Formative Ascriptors, Truth, and Knowledge}

Formative ascriptors are not lexicative even though they contain lexicators, for they do not designate, appraise, or prescribe. If one is told that it will either rain or not rain tomorrow in Chicago, he is not led by the signs of his communicator to expect one kind of weather rather than another, nor to give preferential consideration to rain or the absence of rain, nor to act in one way rather than another to his environment. Nevertheless such a formative ascriptor is "about" the weather, "says something," is true or false, can be believed and be known to be true.

What such an ascriptor signifies is that rain tomorrow in Chicago or its absence tomorrow in Chicago are alternatives. In spite of its similarity to statements (such as 'it will either rain or not snow tomorrow in Chicago') we have felt it wise to call an assertion of a formative ascriptor by another name, i.e., a formulization, restricting statements to the assertion of ascriptors which designate. A formulization then asserts a formative property of something. Such properties as alternativity, conjunctivity, singularity are as "objective" as any other properties, but just as the property of being a valuata is objective only in relation to preferential behavior, so formata are objective properties only in relation to sign-behavior (that is, to interpretants). That formata have this status, and are not characteristics of situations, is shown by the fact that

${ }^{1}$ Mind, Self, and Society, p. $126 \mathrm{n}$. 
any object or situation may be an "or" object or situation with respect to some sign-process (such as 'That is either gold or it is not gold').

Since a formative ascriptor ascribes a formative property to something or other, it denotes if the something or other has the property ascribed. A formative ascriptor is, therefore, in common with other ascriptors true or false (a $\mathrm{T}$-ascriptor or an $\mathrm{F}$-ascriptor). But since an ascriptor is true (is a T-ascriptor) if it denotes, it follows from our criterion for a formative ascriptor that the truth of a formative ascriptor requires as its sole condition the truth of its component antecedent ascriptor or ascriptors.

To know that an ascriptor is formative, just as to know that an ascriptor is designative, is not the same thing as to know that it is true. Both require evidence, but in the first case the evidence is merely as to the signification of the ascriptor while in the second case evidence is required as to whether the ascriptor denotes. In proportion to the evidence that an ascriptor is such that its truth requires as its sole condition the truth of its component antecedent ascriptor or ascriptors, we have knowledge that (know it to be true that) the ascriptor in question is formative; in proportion to the evidence that the antecedent ascriptor (or ascriptors) of a formative ascriptor is true, we have knowledge that (know it to be true that) the formative ascriptor in question is true. These two questions are often confused, ${ }^{\mathrm{G}}$ even by able logicians, with the result that "formal truth" is sometimes supposed to be a different kind of truth than "factual truth," and to be known by some unique kind of "a priori" knowledge. Formative ascriptors are not lexicative or synthetic ascriptors, to be sure, but there is no difference in kind in knowing whether an ascriptor is formative or lexicative, or whether a formative or a lexicative ascriptor is true.

Let us illustrate the situation by an example at the human level where we may suppose a formulation of the signification of the signs in question. We want to know if that 'that black berry is black' is a formative ascriptor. Let the signification of 'black berry' be $A$ and $B$ and $C$ (that is, anything which meets conditions $A$ and $B$ and $C$ is a denotatum of 'black berry'); and the signification of 'black' be $A$. We now see from an observation of these formulated significations that if 'black berry' denotes, then 'black' denotes, for the conditions of denotation for 'black' 
(namely, $A$ ) are met if the conditions of denotation for 'black berry' (namely, $A$ and $B$ and $C$ ) are met. But then the ascriptor as a whole denotes if 'black berry' denotes, for an ascriptor by definition denotes if the dominant sign denotes what the identifior or the subordinate ascriptor denotes. Hence 'that black berry is black' is an analytic formative ascriptor. But if we take the ascriptor 'that blackberry is black' we find that in normal English usage the signification of 'blackberry' is such that its denotata need not be black (as when the berry is young). Hence this ascriptor is not formative but lexicative or synthetic.

To know now whether ascriptors are true takes us beyond evidence as to their signification and requires evidence as to denotation. To know the truth of 'that blackberry is black' we have to have evidence that something is a blackberry and that it is black. While to know the truth of 'that black berry is black' we need evidence only that what is identified is a black berry. The case would be less trivial if we took as our example here 'that black berry is colored,' since this would make clear that we need no additional evidence to determine that a berry is colored beyond what is required in determining that it is black.

In both cases truth requires denotation. And in both cases knowledge requires evidence; whether the knowledge concerns the mode of signifying of an ascriptor or its truth. If there was nothing existent there would be no denotation-and no truth, factual or formal. If by some miracle there were still ascriptors it would be true that a given ascriptor was formative, and true that it would be true if its antecedent ascriptors were to denote. But true it would not be. And if there was no evidence, no ascriptor, formative or lexicative, would be known to be true. But there is a world and it furnishes evidence as to whether our signs denote. And if there was no such world, then there would be no signs, and no knowledge, and no truth. Not even the truth that there was nothing. The reader is free at this point to utter any appraisor he desires. I am satisfied to smile.

\section{Logico-Mathematical Discourse}

If formative discourse be considered as discourse in which formative ascriptors are dominant, we are led to expect four main types of forma- 
tive discourse distinguishable in terms of the four main uses to which signs can be put. It must be admitted that illustrations of these are difficult and somewhat arbitrary, since our common language can hardly be expected to reflect accurately the complex and subtle distinctions which are involved. Nevertheless, the distinctions are in a vague way present there, and an illustration of them will show that nonlexicative ascriptors can be important.

The first type of formative discourse-formative-informative discourse-causes the least difficulty. For it would be very generally admitted that many sentences commonly called "logical" and "mathematical" are of this nature, though the definition of 'logic' and 'mathematics' is a matter upon which there is a great difference of opinion. So we may regard logico-mathematical discourse as giving an illustration of formative-informative discourse, provided we are clear that we are not defining such discourse or defining the relation of logic or mathematics to this discourse.

'If men are animals, and animals are mortal, then men are mortal' may be taken as an instance of the kind of discourse we have in mind. Tested in terms of our preceding distinctions and methods, this compound ascriptor is formative and analytic in English, regardless of whether the component ascriptors are formative or lexicative. A consideration of the interrelationship of the significations of the component signs shows that any situation which fulfills the conditions of denotation of the antecedent ascriptors has thereby fulfilled the conditions of denotation of the consequent ascriptor. So we know that men are mortal if they are animals and if animals are mortal; no evidence in addition to that attesting the truth of the premisses is necessary to attest the truth of the conclusion. The ascriptor as a whole is accordingly an analytic formative ascriptor, differing only in complexity from such an ascriptor as 'That red thing is colored.' The same is true of ' $2+2=4$.' The analysis of this ascriptor shows that in a certain language the significations of the signs in the sign combination are such that any situation which meets the conditions of denotation of ' $2+2$ ' thereby meets the conditions of denotation of ' 4 ,' and vice versa. ' $2+2=4$ ' is, therefore, in the language in question an analytic formative ascriptor.

Now such formative ascriptors may be used for various purposes. 
We might use ' $2+2=4$,' for instance, to cause someone to appraise us positively as learned, or to incite a certain habit of substitution in someone. Neither of these uses would, however, differentiate what is often intended as logico-mathematical discourse; such discourse seems to be "informative." But informative of what? Informative of the speech habits of certain persons?-but then logico-mathematical discourse would be a part of the social sciences. Informative of features of the world, such as that 2 volumes added to 2 volumes give 4 volumes?but 2 quarts of alcohol added to 2 quarts of water do not give 4 quarts. The remaining alternative seems the acceptable one: such formative ascriptors constitute logico-mathematical discourse when they are used to inform concerning the formative ascriptors of the language in which they appear. Since they are formative ascriptors they do not designate either the world or language; they may, however, be used to inform their interpreters concerning their status as formative ascriptors, and when so used they are illustrative of logico-mathematical discourse. ${ }^{\mathrm{H}}$

This discussion, fragmentary though it is, throws some light on why logico-mathematical discourse is significant. It is important, first of all, to be informed of which ascriptors are formative and which lexicative so as not to confuse the two; otherwise we are confusing knowledge of our language with knowledge about the rest of the world. We may then think that Euclidean geometry answers the question as to the character of the space of our physical world, or that ' $2+2=4$ ' tells us something about the results of combining objects in the world, or that deductive argumentation by itself suffices to determine what is true. But this distinction, important as it is for intellectual clarity, is secondary in importance to the way that formative ascriptors aid the organization and testing of lexicative ascriptors. For it is by formative ascriptors that we see certain interrelationships between our lexicative ascriptors. By finding a small number of ascriptors from which the other ascriptors that we accept follow as implicates we attain organization, compactness, and generality in our knowledge. And by the reverse process, in finding what other ascriptors we are committed to if we accept certain ascriptors as premisses, we are often in a better position to test the conclusions in a more direct way than we can test the premisses, and in this procedure indirectly to test the premisses themselves. So both in the organization 
and in the verification of our knowledge formative ascriptors play a significant role. ${ }^{I}$ The kind of information they provide us is for these reasons of high importance, and not of less importance simply because formative ascriptors do not and cannot give us lexicative knowledge. Logico-mathematical discourse informs us of the portion of our language which is composed of formative ascriptors. And in the organization and the facilitation of knowledge it is a powerful aid toward the advancement of the lexicative modes of signifying and the purposes to which they minister.

\section{Rhetorical Discourse}

It is more difficult to find examples of discourse which is formative in mode of signifying and valuative in aim. 'Children are children,' 'Women are women' are obvious examples; 'A good will is alone good' (in Kant's language) and 'The nature that craves change is bad' (in Aristotle's language) may perhaps serve as less obvious illustrations; the ascription in some religions of contradictory predicates to god would supply examples of contradictory formative ascriptors. Such sentences are formative ascriptors; in these examples, however, they are not used to give information about a language but to call out valuations: children are not to be condemned for acting as children and not as adults; persons who crave change are persons to be disapproved of; moral approval should be given to a person in terms of his intentions and not in terms of the consequences of his acts; god transcends and is superior to the contradictions of mortal and finite existence. Another set of illustrations can perhaps be drawn from the use of formative ascriptors to induce valuations of ascriptors themselves: the conclusion that a person draws from certain premisses is opposed on the ground that his reasoning is "bad" since his argument is "incorrect": "It does not follow that all signs are lexicators just because all lexicators are signs." Here an analytical formative ascriptor (of the type "all $x$ is $y$ ' does not imply 'all $y$ is $x^{\prime}$. ') is used to call out disapproval of the statement 'all signs are lexicators.'

There is, however, no term in general use to name discourse of the type represented by these examples. In past times 'normative logic' 
would have seemed appropriate for some of the illustrations, since "logic" was often held to be the "art" of correct and incorrect (good and bad) reasoning. Today, the term 'logic' has so many significations that its employment in this connection would be misleading. The same is true of another term which suggests itself: 'dialectical discourse.' The term we have chosen-'rhetorical discourse'-is not without disadvantages, for 'rhetorical' is often used to designate any pompous or bombastic discourse. Nevertheless, the term is defensible to some extent since certainly one main characteristic of rhetorical utterances is their use of formative ascriptors to induce valuations of persons or events or ascriptors. In an oft-quoted funeral oration of Gorgias, clearly delivered to awaken praise for men whom others were condemning, occur these lines: "For the virtue of these men was a divine possession; their mortality was human. Frequently they preferred the clemency of equity to the harshness of law; frequently, too, the righteousness of reason to the rigidity of codes. For this they held to be the most godlike and most universal code; in the right place to do aright and to speak aright, to keep silence aright, and to bear aright. It is a law of nature that the strong shall not be hindered by the weak, but that the weak shall be ruled and led by the strong; that the strong shall go before and the weak shall follow after." Here certain deviations from rigid laws and codes are made to appear praiseworthy by the use of two implicit formative ascriptors (that is, formative ascriptors in the language of Gorgias): the right is relative to specific situations; the right is relative to the strength and weakness of persons. These analytic sentences are presented, to be sure, as if they were appraisively or designatively true statements, but that itself is simply a device to increase the adequacy of the discourse with respect to the purposes for which it is intended. And the rhetorical character of the oration is certainly due in part to the valuative use to which the formative ascriptors are put.

A similar situation is found in cases where the purpose of the discourse is to call out acceptance or rejection of an ascriptor by using formative ascriptors in the argument by which the conclusion is reached. Simplicius formulates Zeno's argument that the arrow shot from a bow does not move as follows: "The arrow which is moving forward is at every moment in a space equal to itself, accordingly it is in a space equal 
to itself; but that which is in a space equal to itself in the present moment is not in motion. Accordingly it is in a state of rest, since it is not moved in the present moment, and that which is not moving is at rest, since everything is either in motion or at rest. So the arrow which is moving forward is at rest while it is moving forward, in every moment of its motion." A detailed analysis of this selection is complicated and not necessary here, but it is evident that it aims to induce approval of the doctrine that the arrow does not really move by the use of the analytic formative ascriptors: 'that which is at a place at a moment is at rest in that place at that moment,' 'everything is either in motion or at rest,' 'that which is at rest at every moment is not in motion.'

In both of these examples we see the temptation which always besets the producers and interpreters of formative discourse: the temptation to see its results as more than formative, that is, as lexicative. It is hard to learn the lessons that no consideration of signification alone establishes denotation and that the presence of lexicators in an ascriptor does not guarantee that the ascriptor is lexicative. So the eternal raising of the ghost of the "synthetic a priori," a ghost which we believe to have theoretically laid. But a recognition of the seductiveness of the temptation to confuse formative and lexicative types of discourse should not lead us to overlook the importance of formative discourse in general, or of rhetorical discourse in particular, as contemporary semioticians have often done. For formative ascriptors can be used for many purposes, of which the informative is only one; rhetorical discourse shows how formative ascriptors can be used to induce valuations, and this use is as legitimate as any other. We will now see that they may be used incitively as well.

\section{Grammatical Discourse}

Illustrations of formative-incitive discourse can be drawn from various fields. A person irritated by the indecisiveness of another person might exclaim: 'Either come or don't come!'-hoping to incite in this way the making of a decision by the other person. Or one might make the utterance 'A moral person ought to try to do the right thing' in order to 
incite some activity in the person addressed which is admitted to be "right." Contradictory formative ascriptors ('Come and don't come!') can be used to paralyze and prevent action. We will, however, confine our illustrations to a special instance of formative-incitive discourse -an instance that may be called grammatical discourse (though other terms such as 'hortative' or 'pedagogical' or 'paradigmatic' might be employed instead).

As a preliminary remark, it will be recalled that one of the conditions laid down for signs to constitute a language was that they be combinable in some ways and not in others. This requirement shows itself in several ways. A language may require that if a sign with a certain signification appears in a sign combination certain other signs must appear; thus there are languages which require that if a person be designated one must also designate whether the person is invisible or visible to the speaker, or that if something is done one must signify the instrument by which it is done, or that a signified action must be signified as completed or not completed. Further, a language will require that certain signs have a fixed place in the sign combinations in which they appear: we can say in English 'John gives the book to Jack' but not 'to book the gives Jack John.' And finally, languages impose various requirements upon the physical characteristics of the signs which occur in various sign combinations, such as the adding of certain endings to one sign when it is linked in certain ways with a sign of a given class ("gender" endings, agreement in person and number between the signs signifying actor and action, prefixes or suffixes distinctive of parts of speech are examples).

Now these requirements concerning sign combinations which are distinctive of a given language enter into the definition of the language itself (or at least of the language at a given time). They become part of the norm, then, as to whether one speaks the language "correctly" or "incorrectly"; they constitute the "grammar" of the language in question. One might be understood in a given communication if he said 'hit Jack John,' intending to inform that Jack hit John, but he would not have spoken English "correctly" or "grammatically." It follows that a table, say, of the declensions of Russian adjectives in relation to the nouns they specify need not be taken as a designative statement that 
all Russians do in fact speak in a certain way, but as a paradigm of the way one "ought" to speak Russian. And so taken, the table of declensions is really an analytical formative ascriptor: 'Adjectives in Russian are declined as follows: _____.' The ascriptor is formative since the signification of 'Russian language' implicates the paradigmatically presented declension of adjectives. Insofar as one uses such a table to incite in himself or in others the acquisition of the habits of combining signs definitive of the Russian language, the discourse in question is formative-incitive, and is not unfairly called grammatical.

In the same way a text book of Russian will give paradigms of the forms of Russian ascriptors, showing the kinds of signs which must appear in them and the order of these signs in the ascriptors. By the use of these forms-which include both lexicative and formative signsone may endeavor to learn to speak and write Russian. A poet's handbook would provide other illustrations. It will give, for example, the rhyme scheme of a sonnet; that this is the rhyme scheme for a sonnet is a formative ascriptor (or at least may be). Used by a person to incite the habits of writing sonnets it would be an instance of what is here called grammatical discourse.

These examples are intended to reinforce the point already made that formative ascriptors are subject to the same variety of uses as are other ascriptors. They may contain designative, appraisive, and prescriptive signs along with the formators which give them their unique character, and they may be used to inform, provoke valuations, and incite actions both with respect to linguistic and non-linguistic matters. A developed and comprehensive semiotic must shake off the prevalent tendency to concentrate undue attention upon the designative mode of signifying and the informative use of signs.

\section{Metaphysical Discourse}

Any use of the word 'metaphysical' takes one into controversial waters. For the term has taken on strongly appraisive signification, being a term of condemnation to some persons and a term of highest praise to others. Often, too, any cosmology is called a "metaphysics." Nevertheless there is a sense of this term, with historical precedent, 
which makes it applicable to a type of discourse formative in mode and systemic in use. There is a long tradition, stemming from Aristotle, which interprets metaphysical sentences as "necessary," as of extreme generality, and as having the peculiar status of being neither verifiable nor refutable by the data of the special sciences-and hence as "meta" physical or "meta" scientific.

The existence of this tradition immediately suggests the view that metaphysical discourse may, in one of the significations of 'metaphysical,' be regarded as formative-systemic discourse, and that its "truth" is the truth of formative rather than that of lexicative ascriptors. It must be at once granted that this interpretation will be rejected by many metaphysicians, who have persistently claimed that their principles, while not empirical, apply to "all being" or "being as such," and are known to be true by other methods than those of science. We suggest that these claims are valid only in so far as they in a vague sense mark the distinction that has been drawn between formative and lexicative ascriptors, and between the differences in the kind of evidence by which knowledge is obtained about formative and lexicative ascriptors; if more than this is meant we believe that the metaphysicians in question fail to make the distinctions which our analysis has revealed. But we will also hold that if metaphysical discourse be interpreted as formativesystemic discourse, it does not thereby lose its importance, but rather that its own unique significance comes into view, perhaps for the first time. ${ }^{J}$

As illustrations of metaphysical sentences we may take such utterances as the following: 'Nothing both is and is not,' 'Change is of the nature of the bad,' 'Only actualities are real,' and their opposites, such as 'Everything both is and is not,' 'Change is not bad,' 'Not only actualities are real.' Now it must be admitted that such sentences do, for some writers, signify lexicatively, and that the line between the lexicative and the formative must be drawn with respect to the specific language in which a sign combination appears. Nevertheless it is also true that for certain writers such utterances are regarded as more than generalizations subject to qualification by additional evidence, since it is often explicitly held that the utterances in question can neither be verified nor 
refuted by the statements of natural science. And when this is the case, such utterances are no longer lexicative, but formative. What then is their importance? In the first place, by their extreme generality, they organize a whole body of formative ascriptors and thereby perform a systemic function. A person who regards only actualities as real will differ basically in what causes or institutions he supports from a person who regards possibilities as real. A person who disapproves of social changes, changeful persons, death and disintegration in all its forms organizes these appraisals in the sentence 'Change is of the nature of the bad' ('either something does not change or it is bad'). And insofar as the resulting ascriptor is formative, the person who accepts this ascriptor has given the widest possible organization to his behavior, since he can no longer be surprised (in case the ascriptor is analytic) or since he always prepared for surprises (in case the ascriptor is contradictory).

This position can be emphasized by considering the differences between the sentences 'Being is material' and 'Being is mental,' assuming that the sentences are analytically formative in the language of the persons who assert the sentences. If this is so the sentences are not designative, so that no specific predictions can be drawn for, say, biology or chemistry. The "materialist" and the "idealist" will have the same biology and the same chemistry; only everything that happens will be called "material" by the first and "mental" by the second; each is in a very general sense "prepared for anything" but not prepared for any specific happening. And the differences of attractiveness of the two formulations may well connect with differences in the appraisive signification of 'material' and 'mental' and these in turn with differences in the personalities or experiences of the two persons. This fits in with the fact that all of the main metaphysical systems appear in the main historical cultures, and even appear at the same time in any one of these cultures. Each can assimilate whatever the science of the time affirms, and neither uniquely confirms itself nor refutes its rivals. This is intelligible if the major metaphysical systems are formative in mode of signifying and systemic in purpose. They give very wide orientations of behavior by generalizing the formative ascriptors of their ad 
herents, and perform for formative discourse a role analogous to that which cosmological, critical, and methodological discourse perform for the other modes of signifying. They organize behavior in such a way that their interpreter cannot be surprised. And this is true even of a metaphysics based on contradictory ascriptors, for to be prepared to be always surprised is itself one strategy for obtaining stability in a surprising world.

When so interpreted as formative-systemic discourse, metaphysical discourse is neither without signification or significance. Rather it becomes one type of discourse among others, objectionable only when its producers or interpreters make claims for it that it cannot fulfill. Since it is not designative, for instance, it cannot claim to give an alternative or rival account of the world to that furnished by science; since it is not appraisive or prescriptive, it cannot claim to give the specific appraisals and prescriptions which life at its actual frontier must always undertake. But as a phase of the organization of behavior, metaphysical discourse has a novel and justifiable place.

A last word to avoid misinterpretation. We have not so far said anything about philosophy, and so have not equated it with metaphysical discourse. The nature of philosophy is a topic to which we will return in the final pages of our study.

\section{Logic and Mathematics}

The identification of the four preceding types of formative discourse does not in itself decide the question as to the signification of 'logic,' 'mathematics,' 'rhetoric,' 'grammar,' and 'metaphysics.' In the present section we will consider the terms 'logic' and 'mathematics,' leaving the others for the following section. We raise these topics here partly because of their own interest and partly because their consideration throws light upon the nature of semiotic and on science. Our aim is to show in a general way how such terms might be incorporated into the terminology of semiotic rather than to insist upon certain definitions of the terms in question.

Logico-mathematical discourse is, in the preceding account, a type 
of formative discourse. 'Logic' might then be synonymous with such discourse or with some portion of it (and so signify the world formatively), or it might be discourse of some other type. Now as a matter of fact the term 'logic' has been, and is today, a term of great ambiguity; this is why we have avoided it (and the adjective 'logical') in building the basic terminology of semiotic. It has been employed to designate various kinds of appraisive and prescriptive discourse (critical and technological), and to designate various kinds of formative discourse (logico-mathematical and rhetorical discourse); it has been regarded as a form of scientific discourse; and it has been linked with semiotic as a whole or with some part of it. In the face of such diversity it might seem wisest to conclude that the term has lost its usefulness for semiotic. But since the term 'logic' (and 'logical') is so strongly entrenched in the vocabulary of workers in this field, it is worth-while to subject it to analysis and to suggest a possible definition of the term in the light of such analysis.

A distinction is current today between an "object language" and a "metalanguage," an object language being any language which is an object for investigation, and a metalanguage being any language signifying some other language; in this usage the terms do not refer to two kinds of language but to a relation between two languages in a given investigation. So if we talked about French in English, French would be the object language and English the metalanguage. Now this distinction itself, as often interpreted, has certain defects. The first of these, the restriction to language, can easily be avoided by recognizing that there may be metasigns (signs about signs) which denote object signs which are not language signs. Hence an object language and a metalanguage are then simply special cases of objectsigns and metasigns (that is, where these are language signs). The second defect is more serious, and springs not from the distinction itself but from the almost universal neglect of the great variety of types of discourse: it is uncritically assumed that a metalanguage is either scientific discourse or logico-mathematical discourse. A metalanguage, however, might be of any type of discourse and its object language of any type of discourse: there can be poetic discourse about poetic dis- 
course, scientific discourse about poetic discourse, legal discourse about poetic discourse, poetic discourse about scientific discourse, logicomathematical discourse about scientific discourse, scientific discourse about logico-mathematical discourse, and so on. Therefore the term 'about' in the phrase 'signs about signs,' is as wide in signification as the term 'signifies,' and signs in any mode of signifying can signify ("be about") signs in any mode of signifying. So we either have to recognize explicitly that any type of discourse can be metalinguistic or restrict the term 'metalanguage' to some specified type (or types) of discourse; we propose the former usage.

This decision has bearings on the interpretation of semiotic itself. For semiotic is "about" signs: its signs are metasigns. But this leaves open the question as to its type of discourse. Semiotic might include all metalanguages or only metalanguages of a given type or types. Since most students in this field would not want to say that all poems about poems are part of semiotic, we propose that semiotic be limited to a metalanguage appropriate to a science.

But at this point it becomes clear that 'science' itself is a term of varying significations. It is seldom synonymous with 'scientific discourse, though closely related to such discourse. When we speak of "the science of physics" we normally include reference to the methods and the instruments which physicists use as well as to their language as physicists, and we would surely hesitate to exclude from this language itself the formative ascriptors which occur in any textbook of physics, though such ascriptors are in our terms logico-mathematical discourse and not scientific discourse. Nevertheless we certainly think of physics as being centrally concerned with the establishment of statements (designative ascriptors) known to be true of physical processes. Accordingly, it seems appropriate to mean by a science the totality of non-linguistic and linguistic factors employed in obtaining statements known to be true about some subject matter (that is, in obtaining scientific discourse) together with the statements so obtained.

Semiotic will then be in this sense a science of signs. Its aim will be to eventuate in scientific discourse about signs; it will in time develop experimental techniques for this purpose, and its language will also 
contain a body of formative ascriptors which considered in themselves belong to logico-mathematical discourse (that is, its language will contain both scientific discourse and logico-mathematical discourse).

It is believed that the preceding analysis suggests an appropriate and useful definition of 'logic': logic is the logico-mathematical portion of the science of semiotic. ${ }^{\mathrm{K}}$ It is formative discourse at the metalinguistic level aiming to give information concerning languages which are the objects of its investigation. Logical analysis of a language consists of the sentences about an object language which are analytic formative ascriptors in the semiotical metalanguage. Logical analysis is then always signification analysis; it takes the signification of signs as given, and neither studies what signification signs have to specific individuals nor the truth or falsity of these signs-such studies belong to the lexicative discourse of semiotic or some other science. Logic is then part of semiotic in the same sense in which mathematical physics is part of physics.

For the term 'mathematics' there are various possibilities open, even if-as seems to us desirable-the term be limited to formative discourse. It might be identified with logico-mathematical discourse (in which case logic is part of mathematics, i.e., the mathematical portion of semiotic). Or it could be restricted to the logico-mathematical portion of semiotic (in which case 'logic' and 'mathematics' are synonymous). Or it could be limited to that part of the logico-mathematical portion of semiotic which deals with the mathematical part of logico-mathematical discourse, however this be differentiated (in which case mathematics is part of logic). Or mathematics and logic could be combined into one discipline, logico-mathematics. Or mathematics could be restricted to logico-mathematical discourse which is not part of semiotic (in which case logic and mathematics are non-overlapping subdivisions of logico-mathematical discourse, logic covering the semiotical subdivision and mathematics the non-semiotical subdivision). I should myself prefer the last alternative; it has the advantage of doing justice to the widely recognized similarity of logic and mathematics by making them both logico-mathematical discourse; it preserves the distinction between mathematics and logic on the one hand and scientific discourse 
on the other; and it "saves" two historically important terms without raising disputes as to which discipline is "part" of the other. When so used, mathematics is not part of semiotic while logic is a part of semiotic; and while mathematics informs about the logico-mathematical discourse of the language in which it itself appears, logic informs about object languages in so far as such information can be given by the formative discourse of semiotic. ${ }^{\mathbf{x}}$

On this proposal the relation of the work of such thinkers as Rudolf Carnap to the science of semiotic becomes clear: Carnap has proposed certain terms for the language of semiotic, has as a mathematician explored a portion of the language of semiotic insofar as it contains such terms (that is, explored a segment of its logico-mathematical discourse) and has as a logician studied various object languages in these terms. The behavioristician has in the meantime been concerned with getting empirically verified statements of actual sign-behavior. Semiotic as an empirical science eventuates in designative-informative discourse about language and non-language signs; semiotic as logic eventuates in formative-informative discourse about languages. Semiotic, then, has its scientific discourse and its logic, and these are no more in opposition than experimental and mathematical physics. ${ }^{\text {M }}$

What some thinkers will feel is left out in this analysis of logic will on this account find its place within technological, methodological, rhetorical, or critical discourse, or in the lexicative portion of semiotic itself. $^{\mathrm{N}}$

\section{Rhetoric, Grammar, and Metaphysics}

Considerations and difficulties similar to those met in the preceding section occur with respect to the terms 'rhetoric,' 'grammar,' and 'metaphysics.' Do these terms designate non-metalinguistic or metalinguistic levels of discourse? If metalinguistic do they fall within or without semiotic? If within semiotic, do they belong to its lexicative portion or to its formative portion?

Rather than carrying through in detail the analysis of these problems we propose to accept a position akin to that often taken in the Middle 
Ages: rhetoric and grammar (the latter we will call grammatic), but not metaphysics, are regarded as parts of the science of semiotic. This, however, raises the question as to whether they are parts of the formative discourse of semiotic (in which case they must be differentiated from logic) or whether they are parts of the lexicative (scientific) discourse of semiotic.

If rhetoric and grammatic are interpreted as formative discourse, the problem is how to differentiate them from each other and from logic. A possible differentiation is the following: logic, rhetoric, and grammatic, regarded as formative-informative discourse (and so as part of semiotic), differ in the kinds of lexicative signs which occur in their formative ascriptors. Thus it might be said that the formative ascriptors which constitute logic contain designative ascriptors, that the formative ascriptors which constitute rhetoric contain appraisive ascriptors, and that the formative ascriptors which constitute grammatic contain prescriptive ascriptors, all alike being used to inform (formatively inform) concerning the languages which are their objects. On this usage " Tomorrow it will rain or not rain' is an analytic formative ascriptor' is part of logic since it is informative of English, contains designative ascriptors, and is itself in the formative part of the language of semiotic; 'I ain't got none' is bad English' is part of rhetoric since it is informative of English, contains appraisive ascriptors, and is itself a formative ascriptor in the language of semiotic; 'One should say in English 'Cows come home' and not 'Cows comes home' is part of grammatic since it is informative of English, contains prescriptive ascriptors, and yet is formatively ascriptive at the semiotic level.

This position would make intelligible the sense in which logic, rhetoric, and grammatic have often been called (misleadingly) "formal sciences." Their "formal" character is unobjectionable: so interpreted they would be formative types of discourse. But as formative discourse they are not sciences, but only parts of a science-that is, of semiotic. Moreover, any ascriptor of the form 'It is known to be true that-' is not itself formative discourse but designative discourse, regardless of whether the form is filled by a lexical ascriptor ('fire burns') or a formative ascriptor (' $p$ or not $p$ ' is analytic in English'), since it rests 
upon non-linguistic evidence that the ascriptor denotes. Therefore, insofar as the formative sentences of logic, rhetoric, and grammatic are known to be true, the sentences embodying this knowledge fall within scientific discourse. But this does not mean that the sentences of logic, rhetoric, and grammatic are part of scientific discourse; they themselves would remain formative discourse within the science of signs.

The other alternative would be to regard rhetoric and grammatic as part of the lexicative discourse of semiotic rather than as part of its formative discourse. In this case rhetoric might be regarded as the study of the adequate use of signs for the realization of various purposes, and grammatic as the study of the grammar of language (that is, the restrictions on the way signs can be combined within various specific languages).

The issues are not as compelling as in the case of the terms 'logic' and 'mathematics' (since few semioticians today give a central place to the terms 'rhetoric' and 'grammatic'), and no decision is imperative as to the way in which the terms 'rhetoric' and 'grammatic' might be incorporated within semiotic. If a choice had to be made, I would prefer the first of the two alternatives (in which case rhetoric and grammatic are parts of the formative discourse of semiotic), mainly because contemporary semiotic tends to adopt other terms which cover what is intended in the second alternative ('descriptive pragmatics' and 'descriptive syntactics,' terms to be discussed in the final chapter). But it is perhaps preferable not to introduce the terms 'rhetoric' and 'grammatic' into semiotic at all; they are not necessary, and if not introduced no qualification has to be made to the proposal that logic be regarded as the logico-mathematical portion of semiotic.

A word as to 'metaphysics.' It would of course be possible to treat metaphysics co-ordinately with logic, rhetoric, and grammatic. But the historical tendency to which we have tried in the main to conform has not done so, and for understandable reasons. Metaphysical discourse, as an instance of the systemic use of signs, is similar in character to cosmological, critical, and methodological discourse, and these types of discourse have never been regarded as falling within (or at least exclusively within) semiotic. So our proposal is not to distinguish 
metaphysics and metaphysical discourse. Certain of the issues suggested by this rather cavalier suggestion will reappear when we raise questions concerning the language of philosophy.

And now a word of explanation. I should feel somewhat disappointed if the reader does not himself experience a sense of disappointment with this chapter, and indeed with the entire treatment of the various types of discourse. Such misgivings, however, are but witnesses to the astonishing complexity of sign phenomena and to the corresponding difficulty of devising a language in which to talk about such phenomena. Our account at least shows that semiotic cannot simply take over the current terminology employed in talking about signs. For these terms are riddled with ambiguities and inconsistencies, as the incomplete analyses of 'science,' 'logic,' 'mathematics,' 'grammar,' 'rhetoric,' and 'metaphysics' has shown. The inconclusiveness which most readers will feel in the analysis comes from the fact that different analyses are possible, each of which is defensible in terms of an appeal to some segment of historical tradition.

What must be made clear, however, is that our attempt to show the relevance of semiotic to the actual complexity and multiplicity of the types of discourse employed in human society has not compromised or weakened the foundations laid in previous chapters. We have not introduced any such term as 'logic,' 'science,' 'poetry,' 'law,' 'religion,' etc., into the basic terminology of semiotic. We have tentatively suggested how some such terms might be introduced into the superstructure of semiotic, but their analysis is secondary to our previous analysis and not vice versa. Semiotic is not committed to any one such analysis, and may dispense with all of them.

What is basic to our argument is the behavioral analysis of signs, the differentiation of the modes of signifying, the distinction of the main uses of signs, and the mode-use classification of types of discourse. Everything else that has been said is superstructure, and here our contention has been merely that it is possible to deal with all sign phenomena in terms of the basic terminology of semiotic, and hence to define any other term signifying sign phenomena in these terms. Our concern in this study is with the foundations of semiotic; the suggestions 
as to the relations of currently employed terminology to these foundations is merely illustrative and suggestive, and in the nature of the case inconclusive. So in the present chapter the important point is the attempt to give a behavioral differentiation of formators and formative discourse, and not the proposals as to how 'logic,' 'mathematics,' 'rhetoric,' 'grammatic,' and 'metaphysics' might be defined on the basis of the fundamental terms of semiotic. 


\section{INDIVIDUAL AND SOCIAL IMPORT OF SIGNS}

\section{The Problem of This Chapter}

G VERY sign involves behavior, for a sign must have an interpretant, and an interpretant is a disposition to a response. But the behavior which is involved in the sign as sign itself occurs within the context of the more inclusive system of behavior of the interpreter of the sign, and frequently within the context of a behavioral system involving a number of organisms. There arises in this way the problem of the relation of signs to the individuals and societies in which signs appear and operate. This topic has been touched upon already in a fragmentary way in the discussions of the genesis of language and the uses of signs. But there the problem was incidental and not focal. Nothing has yet been said in any comprehensive way as to the individual and social conditions for the appearance of signs or the effect of signs upon the individual personality and upon society. And here a very extensive field for semiotic opens up. Not merely do signs have a certain signification at a given moment, but they have this signification only within the particular life history of their interpreters; and their appearance affects for good or ill the further life history of these interpreters. The genesis and the effects of signs ("healthy" as well as "pathic"), with genesis and effects considered both from the point of view of individuals and societies-this is the field with which we are now concerned.

And once again we must raise the warning that not too much can 
be expected. For the answers to such problems would constitute a large part of the content of the science of semiotic, and we are only at the threshold of such a science. We have begun to develop a language to talk about sign phenomena; we are not yet in a position to formulate the laws of such sign phenomena. For this reason anything said at the present moment must be mainly a clarification of problems and the indication of a method for their solution.

This does not mean that there does not now exist a large body of material bearing on the relation of signs to individual and social behavior. For one can point almost without end to studies on the language of children, on ritual, magic, and myth, on the individual and social roles of the arts, on the non-language and language symbols of neurotics and psychotics, on the way signs serve social organization. Indeed, there is hardly any study in psychology or the social sciences which does not bear in one way or another upon signs in relation to wider behavioral systems. One can even say that certain large generalizations as to the crucial role which signs play in the development of the most distinctive features of human personality and human society are almost universally accepted by those who have worked through even a part of the vast body of material presented by contemporary psychology, sociology, anthropology, psychiatry, and linguistics. Nevertheless, the total result on the science of semiotic is not impressive. And the reasons are obvious: there is as yet no wide acceptance of a common terminology to talk about sign phenomena; most of the material gained on such phenomena has been incidental to other research; the point of view under which these psychological and cultural studies are made has seldom been behavioral; the semioticians as such have too rarely extended their interests beyond the signs of science and logic. The present situation, then, is about as follows: the material relevant to the problem of this chapter, while abundant, is generally couched in terms of an uncritical, unprecise, and overly simple semiotic vocabulary, while the semioticians (even when behavioristicians) have not devoted themselves sufficiently to a study of the personal and social contexts within which signs function.

An effective co-operation between semiotic and the social-psychological disciplines is then largely a matter for the future. Before general 
empirical laws are obtained which will permit predictions in this field much deliberate experimentation on animals, children, and adults will be necessary, and where experimentation is not possible, careful observation deliberately oriented within the framework of, and for the purposes of, semiotic is essential. Already there is a recognition of the need of such co-operation between the analytical and the empirical poles of semiotical studies. Both Tolman's and Hull's laboratories have recognized that their programs for behavioral science involve the study of signs, and the general behavioral principles they have obtained should point the way in the near future to laws for signbehavior; linguists such as Sapir, Bloomfield, Andrade, and Gardiner have progressively tended to link linguistic studies with psychological and social studies; a long line of psychiatrists (among them Head, Freud, Meyer, Sullivan, Goldstein, Masserman) have come to see the crucial place which signs play in personality disturbances; Lasswell and his co-workers are making earnest attempts to apply statistical methods to the analysis of communication; anthropologists such as Malinowski, Warner, Chapple, and Coon have not only recognized the great dependence of culture on signs but have begun to analyze specific cultural phenomena (ritual, myth, law, money) in semiotical terms. And the semioticians have likewise begun to extend the province of their studies, preparing categories applicable to all forms of sign phenomena, and organizing in a preliminary manner the data obtained by workers in all of the fields which have been mentioned. One evidence of this is the work of Ernst Cassirer and his followers (such as Urban and Langer); another and more behavioral emphasis is represented by the work of George Mead and such studies as the present volume which continue the general direction of his investigations.

This situation sets the task of the present chapter. It cannot pretend to give laws as to the relation of signs to individual persons or societies. It cannot even aim at a systematization of all the data garnered on these topics by various investigators. But it must attempt to show how the problems concerning the individual and social contexts of signs present themselves at the present stage of development of semiotic, and suggest in a large and tentative way the healthy and pathic effects of signs on individual and social behavior. This may serve 
to advance more genuinely scientific work on these problems, and in this way to prepare the further development of semiotic itself.

\section{The Significance of Non-Vocal Signs}

A proper understanding of the way signs operate in individual and social behavior demands an explicit recognition of the importance of signs other than those produced by the vocal chords and heard by the ear. Spoken-heard signs are so central in the life of man, and offer such opportunities for study, that they have almost dominated the interest of semioticians. Such signs alone are often called "language," and since man has been regarded as the linguistic animal, it has seemed natural to concentrate on them. But this, if carried too far, is a great error, and one from which semiotic must now free itself. For not only does the spoken-heard language depend in crucial places on other signs, and itself give rise to signs which are not spoken and heard (post-language signs), but such language is not coextensive with all signs drawn from sounds and has never supplanted its great rivalvisual signs. An age in which printing, photography, painting, film, and television have an important place will call for a semiotic which has not neglected the visual sign; music lovers will rightly ask the sign status of musical sounds; and students of human nature will seek insight into the role of those signs which play such a prominent place in "thinking" and yet which are not spoken or heard. A comprehensive semiotic must then do justice to non-vocal signs. ${ }^{\mathbf{A}}$

One of the advantages of our basic terminology lies in its generality: it allows us to talk of all signs, whether language or non-language, and whether drawn from auditory, visual, tactile, or proprioceptive stimuli. So in dealing with non-vocal signs no new principles are involved. For the moment we wish to draw attention to visual signs and to iconic signs (whether or not visual).

Visual stimuli compete with auditory stimuli as the main source material for signs. It is perhaps useless to try to give one a place of dominance over the other. The enthusiasts for the spoken word have often tried to derive every other form of sign from spoken-heard languages. This may be true for some of the other forms of language; 
it is hardly true for signs in general. Signals, as we have used the term, can be auditory without being language signs, and the psychological mechanisms by which a sound becomes a sign apply equally well, and as directly, to visual stimuli. It seems probable that sounds and sights are two independent sources of signs and that though each has used the other where it can (as picture drawing became in part subordinate to speech in the development of writing), the two developments have gone on parallel, one or the other becoming relatively more important at certain stages of human history. Semiotic should neglect neither, nor the complexity of their interrelationships.

A light which signals food to a dog is as "primitive" as a sound signal; and interpersonal relations are as much determined by the signs gained by the sight of other persons (manner of dress, gestures, facial movements, physical appearance) as by the sounds he utters. I recall once in Poland an elaborate communication in which not a sound was uttered: a young boy and his father stood near me on a crowded train; the father moved down the car without the boy noticing this movement; the boy then looked for his father, did not see him, became panicky; I touched the boy on the arm, pointed to his father, and the boy ran off, happy; his father smiled, and tipped his hat, and I smiled back. The situation was "clear" to all of us, replete with signs, and soundless. Although what is "perceived" in such institutions is certainly in part due to the prior occurrence of spoken language, ${ }^{\mathbf{B}}$ such facts do not warrant the general conclusion that visual signs are less primitive than auditory signs.

An iconic sign, it will be recalled, is any sign which is similar in some respects to what it denotes. Iconicity is thus a matter of degree. It can obviously be a property of auditory and visual signs alike. Spoken language contains some sounds which are clearly iconic ("onomatopoetic"); the extent of its iconicity is a difficult matter to determine. Some linguists have claimed this quality for certain vowels, ${ }^{\mathrm{c}}$ and the poet certainly at times reproduces in the rhythm of his words movements of the objects which the poem signifies. A sound may be iconic of things other than sounds. Max Wertheimer has shown that there is a large agreement between persons who are asked to pair musical improvizations with persons or with paintings. The similarities in 
such inter-sensory phenomena are often difficult to isolate, but are indubitable; they are important in considerations of the possible range of signifying in music.

Visual signs often show iconicity to a very great extent, though of course a visual sign need not be iconic. Photographs, portraits, maps, roadmarkers, models are iconic to a high degree; dreams, paintings other than portraits, musical scores, moving pictures, the theatre, rituals, pageants, the dance, dress, play, and architecture are iconic in varying degrees. Such phenomena make up a large part of human culture, and indicate the possibilities, and the problems, which a developed theory of signs must embrace.

The general importance of the icon follows from its nature: the interpreter highly activates in himself his dispositions to respond, and can by the study of the icon acquaint himself with certain properties of what is signified. And since the icon can signify in any mode of signifying, it can be a powerful aid, by its concreteness, in facilitating any kind of sign-process directed to any purpose for which signs can be used. And in ritual, play, the theatre, and the dance, human activity is itself partially iconic, and as such is partially satisfying of the needs by which it is provoked, while at the same time intensifying the dispositions to response which are necessary for the fuller satisfaction of these needs.

\section{Are the Arts Languages?}

That there are non-vocal languages is not generally disputed, though there is dispute as to the extent of such languages and their relation to spoken language. Hardly anyone would deny that the deaf and dumb communicate linguistically; most persons would include writing within language (though L. Bloomfield would not do so). And in such instances the claim that such languages are genetically later and so historically dependent on spoken language is convincing. How do matters stand with the arts? It is common to speak of the language of music and painting; is the term 'language' in such cases to be taken literally or metaphorically?

Susanne Langer has recently argued (in Philosophy in a New Key) that music and painting, while sign phenomena, are not languages, 
since they have no "vocabulary" - that is, no body of signs with an assignable signification. From our point of view (and probably from hers) the determination of whether the arts are languages (lansign sets) is of less importance than the investigation of what they signify and how they signify it-topics upon which she has much to say that is illuminating. Nevertheless I think that the case for the linguistic character of music and painting can be maintained with some plausibility if the iconic sign is made central (though not all-sufficient) in the analysis. ${ }^{\mathbf{D}}$ For it is no more necessary that the single tones of music or the single lines of a painting be signs than that the vocabulary of a spoken language be sought in the individual sounds phonemes out of which its signs are built up.

In the case of "realistic" painting and "program" music at any rate it seems clear that recognizable objects (such as painted chairs or persons, or the tonally "painted" portrait of an object or person) furnish a vocabulary of signs which are then combined "grammatically" in various ways according to the style of a particular school or artist. It is true that such icons may become very general, as in the case of the "formal" or "automorphic" kinds of painting and music, but generality of signification is not the absence of signification. I have asked many persons, for instance, what kind of situation Stravinsky's Rite of Spring might denote (that is, what is its signification). The answers are various: a herd of wild elephants in panic, a Dionysian orgy, mountains being formed by geological processes, dinosaurs in conflict. But there is no suggestion that it might denote a quiet brook, or lovers in the moonlight, or the self's tranquillity. "Primitive forces in elemental conflict" - such is the approximate signification of the music, and such conflict is presented iconically in the music itself.

A piece of music or an individual painting, looked at in this way, builds its material mainly into iconic signs and these into a single compound icon, and can in this way signify whatever can be iconically signified (painting is not, for reasons already mentioned, limited to signifying what can be seen nor music to what is heard). And I see no compelling reason for not regarding the arts as languages, dependent in part upon spoken language (so that the works of art often become interpersonal post-language symbols), less adequate than spoken lan- 
guage for some purposes of communication but more adequate for others.

It is, however, quite correct, as Langer insists, that the arts (whether conceived as languages or quasi-languages) are not to be looked at merely as being composed of expressive or emotive signs. For an iconic sign is no more and no less expressive than a non-iconic sign; it can, like the latter, signify in any mode of signifying. An icon can designate, but it can also appraise or prescribe. A portrait of a person, together with the name of the person, is, as Peirce held, no less a statement than the verbal description of a person. And by the same token something can be appraised by icons (can for instance be caricatured or idealized in paint or sound), so that the arts may signify in the appraisive mode They may also signify prescriptively, since a command can be given visual form or signified musically by the use of sounds similar to the speech melodies of spoken imperatives. Finally, the arts may signify formatively: not only may they include formators but they may in subtle ways link signified properties as both pertaining to a given object and as not pertaining, and thus at least approximate the formative ascriptors of speech and writing.

Such arts as music and painting may then signify in any of the modes of signifying. And since they can be put to various uses, they can illustrate in various degrees all of the types of discourse which have been distinguished. Painting or music, for example, can be designatively informative, appraisively valuative, and so on. Hence a painting or a piece of music can in principle be scientific, poetic, mythological, religious, and the like, terms which we do in fact frequently (and correctly) employ in this way.

The differentiation of music and painting from other languages does not lie, then, in what is signified or how it is signified but in the dominant role which icons have in the signifying. And if the strength of the iconic sign lies in its ability to present for inspection what it signifies, its weakness lies in the fact that it can only signify what is like itself-a field much wider than might be supposed but far less than human needs require. The iconic sign is ill-adapted to the identification of space and time, so that music and painting are not satisfactory material for scientific discourse-drawings and diagrams being brought 
in more as auxiliary devices to the verbal language than as complete carriers of scientific statements. Nor are prescriptions easily made by icons; the primary religious literature of the world is not adequately formulable in music and paint alone. And formators are clumsily handled in other media than speech or writing. The result is that music and painting have shown themselves most indispensable for appraisive-valuative discourse, since they can embody vividly and concretely in their icons the very characteristics of the objects which in their appraisive capacity they signify as valuata.

This situation throws light on but does not completely resolve the status of the "fine" arts, or art in the honorific and distinctive sense. Iconicity itself is not an absolute criterion of the fine arts, for a novel may be as much a work of art as a painting, and a painting of an object may be scientifically reliable and yet esthetically bad. The common feature of the fine arts of various linguistic media would seem to lie primarily in their valuative use of signs which signify goal-objects, with the additional requirement that the way the signs are employed must awaken a positive valuation of themselves as goal-objects (that is, be at least a part, and perhaps in the limiting case the whole part, of their valuative use). No sign is as such "esthetic" and the attempt to isolate the fine arts by isolating a special class of esthetic signs seems now an error. ${ }^{E}$ The icon naturally has an important place in the fine arts because the valuative aim is more surely reached if an object can be presented for inspection which itself is prized and which iconically embodies the very characteristics of a goal-object concerning which the aim is to induce valuation. So that even fiction and poetry, in the images they induce, and in the appropriateness of their style (or "form") to subject matter ("content"), have to a noticeable degree iconic characteristics. But they are also evidence that the arts are not limited in their valuative aim to the use of icons.

The separation of the fine arts within the arts-however made in detail-is less important than the recognition that non-vocal signs occur in all modes of signifying and are used for all purposes. Ritual (or the theatre or the films), for example, is not a special type of discourse, but an iconic performance of actions which may minister to a great number of individual and social goals. If it may become at times predomi- 
nantly "esthetic," it may also serve to inform its participants and onlookers, or to determine their valuations, or to incite in them specific courses of action. From such a perspective the way is open for a just appreciation of the roles which non-vocal signs (visual or non-visual, iconic or non-iconic) perform in the life of individuals and societies.

\section{Effects of Personal Post-Language Symbols}

Speech, when attained, itself makes possible a vast extension of signprocesses: it may, as we have seen, give to perceived objects a signification first attained by language, and it may give rise to languages (such as that of the deaf and dumb, or the highly developed fine arts) which would not otherwise appear. All such signs are in a large sense of the term "post-linguistic" in that they are dependent upon language for their appearance.

By 'personal post-language symbol' we referred to a special class of such signs, primarily those which are symbols drawn from the proprioceptive stimulation resulting from speech. In talking aloud, for instance, the organism not merely hears the sounds it produces, but receives stimuli from the responses which it makes in and while producing sounds, and such stimuli may in turn become symbols (signs substitutive for and synonymous with other signs). Since such symbols are dependent on speech (and so are post-language symbols), they are often referred to by such terms as 'talking to oneself' or 'subvocal speech'; strictly speaking, however, they are not language signs since they are not stimuli to other interpreters, and the use of such terms as 'speech' and 'talking' with respect to them obscures the important point that they may come from any internal stimulus of the organism and not solely from the movements of the vocal chords. The operation of such post-language symbols is closely connected with "thinking," if indeed thought and the operation of such symbols are not the same thing. ${ }^{\mathrm{F}}$

The importance of such symbols can be best seen in terms of the general significance to an organism of the ability to produce signs. In so far as an organism simply responds to features of its world or itself as signals it is largely at the mercy of events. True that even in this 
case the organism is responding with respect to a wider world than is immediately present, since its response is in terms of what is signified by the signals it receives. When however an organism produces symbols which in turn influence its behavior, its dependence on events for the occurrence of signs is correspondingly (though not entirely) reduced; it becomes in a heightened sense autonomous and self-stimulating. The development of spoken language marks an enormous step in this process: things may be signified in their absence, and the individual may utilize for his goals the experiences, the advice, and the co-operation of other individuals. Personal post-language symbols carry further in certain ways the process of autonomous self-stimulation, for they advance the point of incidence of signs upon behavior. The response involved in the production of sounds is already a welldeveloped response, but if stimuli from the early stages of this response or of other attendant responses become substitute signs for such sounds, then these signs operate earlier within behavior, with great rapidity and with great economy of efforts. Personal post-language symbols allow in this way the maximum possibility for an organism to influence by signs the direction of its behavior.

If we mean by 'freedom' the ability of an organism to direct its behavior by signs, then the highest degree of freedom is found in those organisms in which post-language symbols have attained the highest level of development. ${ }^{G}$ Mead stresses in a number of places that the significant symbol (which, as we have seen, covers our language signs and post-language symbols) allows man to become a "self-conditioning" being, a fact which he thought the existing doctrines of the "conditioned response" failed to account for. The present account can incorporate and explain such self-conditioning. For the person who is planning a shopping tour can by language and post-language symbols signify to himself the consequences, say, of going first to one store rather than another, and through such signifying "condition" himself to respond to the door of the place in question, determining whether he will enter it or pass it by when he encounters it. In the same way a person mạy determine how he will act with respect to certain signs: by signifying the reliability, or adequacy, of certain signs or the purpose for which someone else is producing a given sign, one may decide to 
ignore the sign when it appears, to act cautiously or boldly with respect to it. In this way the effect of a given sign on behavior may be dependent in subtle and complex ways upon the signs which intervene before the incidence of the sign in question, or before the stimulus for which it is preparatory. ${ }^{\text {H }}$

Such considerations clarify the difficulties which confront the extension of behavioral studies to the human person, and offer counsel to such studies. In a number of experiments on human beings the results show marked divergencies from similar studies on non-human animals. In the face of this behavioristicians tend to shy away from human beings and turn back with relief to rats and cats and dogs and apes. The moral would seem to be that significant advance in behavioral studies of human persons requires that behavioristics conquer for itself language and post-language sign phenomena. When this is done, the possibility of finding "laws" for the behavior of human individuals will be greatly advanced. For non-human beings seldom produce the signs which influence their behavior, while human individuals in their language and post-language symbols characteristically do this and to a surprising degree. Here is a basic difference between men and animals, and until behaviorial theory develops a semiotic adequate to this difference it will remain what it is today: a careful study of animals and a pious hope for a science of the human person.

\section{The Pathology of Signs}

Students of plants and animals are accustomed to distinguish between "healthy" and "pathic" events. George K. Link has expressed this difference in such a way that the terms-so frequently appraisive-are given designative signification. Health, he writes, is a state of the organism "in which all structures and activities are so harmoniously integrated and regulated that easy maintenance of the whole organization is continually insured," while "injury, or disturbance of the ideal state of functioning, is the essence of all pathic events." I He has since taken one further step in the isolation of the pathic in order to avoid making every case of the death of organisms pathic: a pathic event is an anomalous or statistically nonfrequent injury. 
In these terms a sign-process, as one kind of event, may be healthy or pathic. "Healthy" or "pathic" signs must be distinguished from "adequate" and "inadequate" signs. An organism with healthy signprocesses may make mistakes: for instance, a dog may be influenced in its behavior by the less reliable of two signs, or a scientist may propound a false hypothesis; such happenings are frequent enough, and though they may be injurious they are not pathic. The signs of an individual are in general healthy in so far as they are subject to correction and improvement; they become pathic in so far as they are anomalously resistant to such correction and improvement. For the most part the signs of an organism are flexible, changing as the needs and abilities of the organism change, and as the environment changes; in this way more reliable and adequate signs replace less reliable and adequate signs. But under certain conditions this flexibility is lost, and sign events become pathic events.

As an illustration at the level of animal behavior we may mention Jules Masserman's experiments on cats. ${ }^{J}$ These cats are first taught to respond to a light as a signal for food in a box in their cage. Then, later, when they respond to the signal by seeking food at the place indicated, a blast of air is let loose at them, and they respond to this by a violent retreat from the place of food. When now the light signal is given, striking behavior results: the animal shows great fear and excitement, cowers in a corner of the cage as far as possible from the food, will refuse food which is available, and may under these circumstances starve itself to death.

One can say that the signal has under these circumstances taken on contradictory appraisive and prescriptive significations. A certain region is designatively signified as containing food and air blasts, and so, because of the preferential behavior of the animal, as being both "good" and "bad"; the animal is as it were "commanded" both to approach the identified place and to get away from it. The result is a paralysis of any effective behavior whatsoever. The pathic element in this situation does not, however, reside in the opposed significations of the signal, but rather in the inflexible way in which correction, or possible correction, of the sign is resisted. Suppose, for example, that the situation is in fact changed so that the air blast is no longer given, 
or is very seldom given; the "neurotic" cat will not undertake behavior by which the former signification of the light is corrected; the light signal becomes a pathic sign. The "healthy" animal would by contrast continue to explore the situation and thus attain such differentiations, and perhaps even attain additional signals as to when the light signal signified both food and blast and when it signified food alone.

The causes of such stubborn resistance to the improvement of sign processes are undoubtedly numerous and complex. But at the human level at least one cause is the partial satisfaction which otherwise inadequate signs may give, or put in another way, is the partial adequacy of the sign for the realization of certain purposes-hence the reluctance to forego this amount of satisfaction by taking the risks which a change in the sign would require. Human beings use signs not only to advance directly certain interests but also to assure themselves that satisfaction of their interests will be achieved and that frustration of their interests will not take place; a signified satisfaction is a kind of satisfaction and a signified frustration a kind of frustration. ${ }^{\mathrm{K}}$ There is thus a certain satisfaction gained by a sign that signifies the attainment of a goal or that relieves the anxiety lest a certain goal be not achieved. Therefore the tendency of a person to hold on to signs which give such satisfaction. This tendency, normal and healthy within limits, becomes pathic when the retention of signs stands in the way of the attainment of greater satisfaction and of more reliable and adequate signs. It is not pathic to cherish the photograph of a loved one; if the photograph comes to prevent satisfactory interaction with the one loved, it has become a pathic sign.

Signs, like other things, become objects of preferential behavior. Since preferential behavior is a concomitant of life, there is nothing objectionable in this. Every normal person prefers some languages to others, some poems to others, some theories to others. But it is characteristic of neurotic and psychotic persons to prefer pathic signs which are unreliable and inadequate with respect to a large segment of behavior because such signs are partially adequate for the attainment of certain goals (such as the temporary reduction of anxiety). In such persons sign-behavior is stubbornly inflexible, and the good has once again become the enemy of the better. The compulsive neurotic who 
must step on every third crack in the walk as a means of gaining selfassurance, or the paranoic who signifies himself an important but unappreciated person, or the schizophrenic who must wear a bit of white ribbon in her hair as a sign of her purity, all relieve their anxieties by signs by which the producers of the signs positively appraise themselves -and so resist violently anything which threatens a change in the signs upon which they have come to depend for assurance of their own importance.

In this way there arises the possibility of explaining the paradoxical situation that signs which normally serve as instruments for behavior with respect to objects may under certain conditions work against adaptive behavior to such objects. The explanation has the merit of not invoking non-behavioral factors, but by showing how certain kinds of false statements, appraisals, and prescriptions give a partial satisfaction to tendencies to behavior which have in one way or another been thwarted. The iconic sign is particularly useful in this respect, for since it is like what it signifies its apprehension gives something of the satisfaction which its denotata would give; at the same time, since iconicity is a matter of degree, the iconic sign permits of being treated in ways which the individual would not do if he recognized the object (say the person) for which the icon is acting as a substitute. Dreams, fantasies, myths, and the arts give abundant examples of these properties of iconic signs as substitute objects for attaining some measure of satisfaction for frustrated actions. ${ }^{\mathrm{L}}$ But icons are not the only signs which function pathically. All signs permit of false statements, appraisals, and prescriptions, and when such signs operate in a behavior context in which their reliability and adequacy is actively opposed because of the partial satisfaction they give, they, whether non-iconic or iconic, function pathically.

\section{Signs and Personality Disturbances}

The previous suggestions as to the way signs function pathically give no basis for the opinion that psychiatry falls entirely within semiotic, or in other terms, that personality disturbances ("mental diseases") are solely pathic sign-processes. Such a position is at times suggested in 
the name of semiotic; it represents an over-simplification which does no service to either semiotic or to psychiatry. What is needed rather is a careful study of the actual extent to which neurotic and psychotic behavior is caused by, transmitted by, and can be cured by signs. ${ }^{M}$ For while the whole explanation of such behavior cannot be couched in terms of signs, signs do figure to an undetermined but important extent in the etiology and therapy of such processes.

With respect to the problem of genesis, Jules Masserman, who accords a prominent place to signs in his behaviorally oriented psychiatry, is explicit on the limitation he imposes with respect to the place of signs in the genesis of pathic behavior: "The motivations of behavior ... may become conflictful, either because of the simultaneous evocation of antagonistic inner needs or through the necessity of diffcult adjustments to complex and contradictory external symbolisms." ${ }^{N}$ The neurotic behavior of the cat may be called out by receiving blasts of air when it is eating without any such signal as the buzzer being involved; or, to use an example suggested by Erich Fromm, a child being held by the mother in feeding may develop conflicting attitudes to the mother merely because of the satisfaction she affords and yet the restraints in bodily motion which she imposes. So even if one neglects personality disturbances due to drugs and organic degeneration, not even all those personality disturbances which come from motivational conflicts can be regarded as due to signs.

That signs may in some cases be a causative factor is not thereby excluded. Harry Stack Sullivan and Erich Fromm have both suggested to me the possible importance of conflicts between the interpersonal and the personal signification of signs. The term 'mother,' for example, may be in a community a term of positive appraisal; a given individual of this community who has come for various reasons to dislike his mother may be caught in conflict between the interpersonal and the personal signification of the term 'mother.' There can in this way arise motivational conflicts which may be caused, at least in part, by the contradictory appraisive and prescriptive significations of certain signs. It would seem possible that certain personality disturbances can be semiotical in origin, and that they may even be intentionally produced in this way-and not only in animals. 
There is even less doubt that pathic behavior, whenever it has arisen, can be perpetuated by signs, for a sign which signifies the situation causing such behavior will itself tend to call out behavior of the same families as those called out by the situation itself. So if the food box or the mother has come to be both an approved and disapproved object, then the light or the word 'mother,' or any other stimuli which become signs of these objects, will in the absence of the food box and the mother call out the behavioral tendencies which they would call out if present. $^{\circ}$ Since at the human level, very complex chains of symbols may arise, it is intelligible that such signs may indefinitely perpetuate personality disturbances beyond the situation in which they arose. Individuals may in fact consciously or unconsciously use signs in order to continue personality disturbances in others or in themselves because of the satisfaction which such disturbances may afford.

If signs play some role in the genesis and perpetuation of pathic behavior, it is to be expected that they may be used to some extent in the alleviation or even the removal of such behavior. Where the signification of a certain sign is a key factor in such behavior, it may be possible to change its signification by the use of other signs-to change for instance its appraisive signification from positive to negative or vice versa. In the same way it may be possible to convince a person by argument that a sign taken to be reliable is not reliable, or that a sign taken to be adequate for the realization of a certain goal is not adequate for this purpose, and in these ways to influence the behavior connected with the sign. Or instead of trying to change the signification of signs directly, signs may be used to change the motivations or the strength of motivations of a person-to get someone, for instance, to dare to tackle a problem that he has been afraid to face; and in this way changes in signs may come about through the new activities of the person himself, a sign previously regarded as reliable perhaps now being seen to be unreliable. The reason why signs may be used for these various ends lies in the fact that the interpretant of every sign is itself a behavioral phenomenon, and so the effect of signs on other signs and on behavior is itself an effect of behavior upon behavior. But since behavior can also be influenced by drugs and by changing the environment in which the organism acts, it would be shortsighted to limit therapeutic tech- 
niques for dealing with pathic behavior to the use of signs, just as it is shortsighted to limit an inquiry into the causes of such behavior to the signs of the person in distress. ${ }^{P}$

Because signs are of such central importance in human behavior, psychiatry must concern itself in a serious way with sign-processes; in doing this it will overlap with semiotic, drawing aid from semiotic as this develops and contributing data important for this development. Semiotic, behaviorally conceived, furnishes a corrective to those semioticians who seem to believe that personality disturbances are everywhere caused by signs and everywhere curable by signs. For since signs themselves can only be understood in the general context of behavior, pathic signs too must be placed within the context of pathic behavior. A behavioral psychiatry is not only possible, but is being developed; and as it develops it will have to do full justice to the place of signs in pathic behavior and to the use of signs in the treatment of such pathic behavior. But it, like semiotic, will be part of the general science of behavior; and while it will utilize semiotic it will not be reducible to it.

\section{Signs and Societies}

A society is a group of organisms in relatively persistent social interaction of such a sort that the behavior of each of the organisms contributes to the satisfaction of the needs of other organisms. In most societies there is some co-operative social behavior, in that there is at least some behavior which requires the conjoint participation of the members of the group for the attainment of the goals of such behavior. Societies show various degrees of cohesion depending on the amount of co-operative social behavior manifested; this varies from group to group and from time to time within a given group. The "totalitarian" societies of today are highly integrated, and in their extreme form attempt to suppress any form of individual behavior that does not support the goals of the society; the earth community is by contrast a very tenuous society in which the goals of national and regional groups greatly predominate over co-operation for the goals of mankind as a whole. A given society may become cohesive in war or in periods of 
threatened danger, and less cohesive as it is secure. But in no case does the relation between socially interacting organisms become entirely cooperative: competitive and symbiotic social behavior is as genuine as co-operative social behavior.

No one, I suppose, maintains that a society is impossible without signs, nor that the existence of signs necessarily produces co-operative social behavior even in the case of socially interacting organisms. But a very widespread opinion has arisen that human society, or at least human society in its cultural aspects, is dependent on signs, and specifically upon language signs for its existence and perpetuation. Malinowski states that "culture without language does not exist"; according to Dewey, language, considered as including "not only gestures but rites, ceremonies, monuments and the products of industrial and fine arts," is "the medium in which culture exists and through which it is transmitted." And such statements are typical of hundreds of similar ones. Since this thesis is, if true, of great importance to semiotic and to the social sciences, it is necessary to be clear as to what it signifies. Unfortunately, this requires agreement as to the signification of the word 'culture,' and such agreement is by no means prevalent among its users. ${ }^{9}$ A sense relevant to our inquiry would be one in which the sphere of the cultural is less wide than that of the social. In all societies, for instance, sexual interactions occur, but they occur differently in different societies, and it is in such differences that differences of culture consist. Not sex relations but the form of sex relations of a given society is cultural; eating is not cultural but the way in which eating takes place in a given society is cultural. Generalizing, the culture of a society may be said to consist of the characteristic ways in which basic needs of individuals are satisfied in that society (that is, to consist of the particular response-sequences of various behavior-families which occur in the society). And a given society, depending on its degree of integration, may show a pervasive and consistent "pattern of culture" or may contain a number of such patterns in a loose or even conflicting juxtaposition.

It is not our task to express an opinion on the origin of cultures, but merely to consider the relation of cultures to signs. In the sense in which 'culture' is defined above, it seems clear that caution is needed: 
it is not evident that a culture is dependent on signs for its origin or even that all cultural phenomena are sign phenomena. The way that one eats, for example, may be due to learning processes which do not involve signs, and the manner of eating need not in any recognizable sense of the term be a sign.

But if cultural phenomena are not necessarily sign phenomena, it may still be true that signs play a very important role in culture and in its transmission. And on this point no doubt is possible. For the language in which people talk, the rites they perform, the monuments they erect, the works of art they make, the devices they utilize to indicate social prestige are all cultural phenomena, and all sign phenomena. For this reason it is possible and revealing, as Chapple and Coon have done in their Principles of Anthropology, to interpret much of the data with which the social scientist deals from a semiotical point of view. In Parts IV and V of this book (entitled "Symbols and Human Relations") they consider rites of passage, rites of intensification, magic, religion, art, money, and law as "series of symbols and their asociated techniques which have meanings held in common by a number of persons," and attempt to differentiate these various cultural phenomena in terms of the signs employed (regarding a law, for instance, as "any rule symbolizing a pattern of interaction which applies to all the members of a group, regardless of institutions"; and a ritual as "a symbolic configuration used to restore equilibrium after a crisis"). This treatment goes far to show the important place which signs have in cultural phenomena, and to suggest the power of semiotic as an organon for the study of man. The semiotic used by these authors is much more adequate than the vague terminology in this field still employed by most social scientists, but though behavioral in its approach, their semiotic is still far from adequate for the purposes of a study of human culture (a sign, for instance, is regarded by them as anything "which sets off a conditioned response"). It is believed that the refinements and distinctions in the science of signs which have been made in this and previous chapters will be useful to the study of man as a cultural being, and that as they are so used in this study the results obtained will in turn greatly help to advance semiotic beyond its present formulation.

Culture, then, does to a large extent exist in the medium of signs. 
Since culture consists of the ways of behaving characteristic of a society, the cultural signs of that society are interpersonal in character. Participation in a culture involves the sharing of its interpersonal signs. These signs are to a considerable extent iconic and, whether iconic or not, are in the main linguistic and post-linguistic. Through such interpersonal signs the members of a society are linked with respect to their statements, appraisals, and prescriptions, and so linked with respect to their behavior. The group can in this way utilize the services of its individual members within complex social acts, determine its goals, relieve its anxieties, celebrate its consummations, and incite itself to action. Human society in its most characteristic traits, like the developed human individual, is dependent in its nature and for its continued existence upon signs, and especially upon language signs and the signs language makes possible.

\section{Social Control}

The recognition that culture is largely a sign configuration suggests at once that the transmission of culture is mainly effected by the transmission of signs from the existing members of the society to the young or to those who enter the society from other societies. It is by instilling in the members of a culture the designations, appraisals, and prescriptions characteristic of that culture that society gains its major control over the individual. For through the induction of the individual into the interpersonal signs of the culture, the individual comes to appraise himself and others and to prescribe to himself and to others in ways born out of, and so congenial to, the society to which he belongs.

George Mead has elaborated the details of this process with great richness and penetration, showing the way in which the "significant symbols" of the culture permit the individual to take the roles of the other members of that culture and to react to himself in terms of such roles. ${ }^{\mathbf{R}}$ Through language signs and post-language symbols the individual internalizes in himself the socially objective process of language communication, his thinking retaining the pattern of conversation, and his self-control through signs continuing in a new and subtle form the techniques of social control. It is in such ways that the phenomena of 
conscience and guilt arise, phenomena which introduce into human pathic and non-pathic behavior a vast range of complications unknown to the behavior of non-human animals and which set limitations to (though they do not deny the importance of) the treatment of human personality disturbances in analogy to the behavioral disturbances of other animals. The human being not only appraises and prescribes for things other than himself, but is constantly engaged in self-appraisals and self-prescriptions of the sort which other members of the society would make with respect to him and to themselves. To his other goals is added the goal of gaining social approval for himself and avoiding social disapproval. And the signs which signify to him such possible approval and disapproval come to cause their own distinctive forms of anxiety and relief from anxiety. So pervasive is this influence that almost anything the individual does-however disruptive or reformatory with respect to society-will be done in the name of the dominant symbols of the society in which he lives.

These socially derived techniques by which the individual carries over into his self-control the forms of social control are so important that every society is especially concerned with the determination of the signs of its individual members. As Mark May has suggested in conversation, such concern is the distinctive feature of propaganda in all its forms; in the widest sense of the word it is the mark of educational institutions. Through its control over what can be said by school and print and film and stage, society attempts to secure for itself the ultimate control over the sign-processes of the individual, and in this way to control its individual members through the signs which will operate in their behavior. The enormous importance in the modern world as to who will control the agencies of mass communication becomes evident. For even if explicit appraisals and prescriptions are not made through such agencies, the mere control of what information is made available to individuals will go a long way toward determining the nature of their own appraisals and prescriptions and hence their behavior.

It should be noticed that there are factors which limit to some degree the social control of individuals by the attempted control of their signs. For the human personality is biological as well as social, and certain biological needs are so pervasive that they cannot with impunity be 
ignored by any special culture. Constitutional psychology, especially as developed by W. H. Sheldon, is performing an important service in calling attention to the place of biological differences in influencing temperament and the personality differences of individuals. And the anthropologist, though inclined to stress the plasticity of personality, has recognized that every culture has its "deviant" members who find it difficult to satisfy their individual needs within the techniques of satisfaction favored by their society. ${ }^{\mathrm{S}}$

A culture, as a preference for certain modes of behavior, involves a preference for certain personality structures rather than others; the kind of person favored in one group may be condemned in another. And those whose personality is looked down upon in a given society constitute one reservoir of resistance to social control and a dynamic source of possible social change. For the sign-processes of a given individual are inevitably subject to correction in terms of the reliability and adequacy of the signs with respect to his own observations and needs, and this is as true of socially implanted signs as of other signs; the deviant individual is simply one in which this process of correction will be strongest. Because of this it is a false picture to assume that "society" simply molds a passive individual to its pattern. Society exists only in the interactions of persons, and the differences in and the variability of persons is an inevitable source of change in society. Though the society makes possible through language the major post-language symbols of the individual, these symbols, when they have arisen, extend the range of behavior of the individual and react in turn upon the language and the culture of the society. The complex society is the condition of the complex individual, but that individual as he arises reacts differently in his social relations and so changes the culture in which he has appeared.

Social control of the individual through the control of his sign-processes is not therefore complete. Nevertheless it can be carried very far, and the extent to which it is attempted and felt desirable will differ with various cultures. A totalitarian society pushes this process to its limit; a loosely organized society will reveal a plurality of various centers competing for control of as many individuals as they can reach; a truly democratic society would aim, as a matter of principle, to enlarge and diversify the sign capacities and resources of its members, assuring them 
access to a wide area of information and encouraging them to test and improve current appraisals and prescriptions in terms of their reliability and adequacy. Only in such a society would semiotic be given a basic place in the educational process, so that the individual would be prepared to resist the exploitation of himself by other users of signs, to avoid pathic signs in his behavior, and to make his contribution to the constant correction and creation of signs upon which a healthy society depends.

\section{The Social Pathology of Signs}

Analogously to the case of the individual it is possible to distinguish socially healthy sign-processes and socially pathic sign-processes, provided that here too we recognize that not all inadequate signs are pathic. In a healthy society signs in all modes of signifying would fulfill the various uses to which signs can be put, and each specialized type of discourse would lend its support to the other types and be in turn controlled by them. Such a society would ground its basic appraisals and prescriptions upon its actual needs and keep them linked to advance in knowledge of itself and its environment. In this way it would seek to reconstruct its preferences and techniques in terms of its knowledge and its needs, and would support all investigations which gave it knowledge relevant to its needs; its sign-processes would flexibly express its needs and aid in their constant reconstruction and redirection; need, knowledge, appraisals, and prescriptions would mutually support and influence each other. At no one time would its signs be completely reliable or adequate, but conditions would exist such that the way was open for the progressive improvement of their reliability and adequacy.

Signs which are socially pathic are signs which are clung to in ways which resist correction of these signs because of the partial satisfaction which these signs give to the social behavior of a group of persons. That there are socially pathic signs seems plausible, though lines cannot be drawn with absolute precision, and though it must be admitted that the area of such signs is less than might at first be expected. If, for instance, the power of the community has fallen into the hands of a group of persons who exploit the community for their own ends, the 
success of this group in prohibiting changes in the sign structure of the society which would be disadvantageous to its members is not a matter of sign pathology; it is with respect to such persons an adequate use of signs. Judged from the standpoint of the society it is immoral and inadequate, but still not pathic. Suppose, however, that the people themselves who are thus exploited, and as a social group, actively resist changes in the very sign structure by which they are exploited, then the situation would have become socially pathic. The signs in question may relieve certain anxieties in the members of society with respect to the social behavior in which they are engaged, and so be cherished for this satisfaction even though the signs hinder or even make impossible the actual realization of the goals of such social behavior itself. And this may be the case for society as a whole and not merely in a group within a society exploited by another group. Signs then become socially pathic only insofar as a number of persons constituting a society resist changes in the correction of signs which function in the social behavior of this group because of the partial satisfaction these signs give to such social behavior.

Illustrations of both these situations are numerous, as the history of religious, political, moral, or metaphysical discourse makes evident. Religious symbols, for example, which are no longer adequate to the social behavior of a society may not only be used by a subgroup within a society to maintain its own privileged status, but the other members of the society may themselves resist changes in these signs because of the reassurance given in these signs as to the status and destiny of the society. And a society as a whole may act likewise, assuring itself of its power and permanence by the repetition of positive appraisals of itself and characteristic prescriptions which no longer correspond to actual realities but which in fact hinder the very power and permanence they are presumed to assure. As the correction of the signs upon which a society depends is actively and unrealistically resisted, the inadequacy of signs-which is universal and compatible with health-passes insensibly into the sphere of social pathology. We have witnessed this process in a startling form in the ideology of the Nazis and Japanese. We are not so likely to recognize the extent to which in ourselves the germs of the same process exist in our religions, our politics, and our 
educational systems. For the same psychological factors which operate in the dreams and neuroses and psychoses of the individual with respect to himself as an individual operate in the sphere of his social behavior to hide from him the social analogues of dreams and neuroses and psychoses.

Pathic behavior at the level of society is as resistant to inspection and correction as it is at the level of the individual. And semiotic has the same possibilities and the same limitations in the one case that it has in the other. Socially pathic behavior, like pathic behavior in general, is not caused solely by signs, but when once it has arisen signs operate as powerful means for its perpetuation. Knowledge about signs may forestall some such socially pathic behavior and aid to some extent in exposing it and breaking it up. But it is utopian to exaggerate such possible influence and to expect the theory of signs itself to produce the signs needed by an individual or a society for an integrated and healthy existence. Semiotic can explain the healthy and the pathic in the realm of signs, and to some extent aid the one and combat the other; but the primary signs which life needs can only be created by those at the frontiers of life itself-by the thinkers and artists and prophets and statesmen whose task is the continual reconstruction of the signs upon which the developed individual and society depend.

\section{Communication, Co-operation, and Conflict}

From the days of Confucius to the present there has been a persistent hope and belief that if men's language was put in order, and this ordered language made a common possession of all men, men would understand each other, society would be stabilized, and conflict would give way to peace.

In its extreme form this position is certainly naïve and indefensible. The sociologist, Robert E. Park, in an article on the relation of communication and culture, ${ }^{\mathrm{T}}$ makes clear that competition between members of a society is as persistent and pervasive as co-operation between them, and that there are "symbiotic" social relations of benefit to organisms which are neither competitive nor co-operative. And from the standpoint of our results, it is clear that signs may and do operate in all 
forms of social behavior, and not only in co-operative social behavior. The question still arises whether the increase of communication (considered as the establishment of sign-processes common to a number of interpreters) does not by and large increase the sphere of co-operative social behavior. Park seems to take this position, since though he holds that competition and communication operate "in relative independence of each other," he states that communication functions "primarily as an integrating and socializing principle." That communication is essential to complex social behavior, and that it primarily serves to increase integration and socialization, is not to be denied; it is well to remind ourselves, however, that it does not always do so.

One indication of this is in the special form of communication in which an individual communicates with himself (that is, the self of one moment communicates with the self at another moment). This occurs not merely in the writing of diaries or the devices by which the present self acts to remind the future self of something, but takes place in a peculiarly important form in the production of a work of art. For in such a production the artist throughout the process is stimulating himself by the stimuli he produces, and at the end of the process in particular he stands over against his work as a member of his audience. There is self-communication in so far as the self is interpreter of what it signified as sign producer; there is social communication insofar as the communication involves interpreters other than the artist.

Now in both these cases the fact of communication may play an integrative role in the selves involved, but need not do so. Let us borrow a term from Abraham Kaplan and call a sign "self-expressive" if the producer of the sign interprets the fact of its production as expressive of himself. The artist may then through the work of art come to a knowledge of the factors in himself that led to the production of the work of art, and by so signifying these factors be better able to incorporate them into a more adequately integrated self. But it is also certain that this may not take place: he may not interpret his products as self-expressive signs but simply enjoy their signification at successive moments of self-communication. If he is a disturbed personality, the art product may itself be a pathic sign, and serve to perpetuate pathic behavior; artists who are insane do not cure their insanity through their 
products. And similarly, if the work of art causes social communication, other interpreters may advance their personal health in this process, but they may not, and they certainly need not, become more co-operative in social behavior as a result of the communication.

The conclusion that communication as such does not necessarily advance either the integration of the selves involved or their social behavior is even more obvious at the level of large-scale social communication. The Irish and the English speak a common language, and more English and Indians speak a common language then ever before, but co-operative social behavior has not been correspondingly advanced. Or in another field, theologians have throughout the centuries defended their respective faiths and read each other's writings; there are few cases where one theologian has converted another. Schools of art and schools of philosophy would supply further instances.

The increase of communication may not only fail to give agreement in valuations and modes of conduct but may actually be used to increase conflict, competitiveness, and slavery. For sharing a language with other persons provides the subtlest and most powerful of all tools for controlling the behavior of these other persons to one's advantage-for stirring up rivalries, advancing one's own goals, exploiting others. Modern propaganda is the witness to this within existing nations; a world language would make the same phenomena possible over the earth as a whole. And semiotic itself, as it develops, will be subject to the same kind of utilization by individuals and groups for the control of other individuals and groups in terms of self-interest.

Such conclusions are in themselves no cause for pessimism. To see danger is to be forewarned; the common and uncritical optimism that any increase in communication is necessarily good is to court the very dangers in question. A world language, the internationalizing of the arts, the reading of the same books-these things are under way and will not be stopped. If they may be powerful forces for the liberation of more and more individuals and for the integration of diverse communities into an earth community, they may also be powerful forces for the mechanization of the individual and the enslavement of society. Which they will be, and how much one and how much the other, will depend upon by whom they are used and for what ends. 
Nor is the relation of the increase of communication to co-operative social behavior one-sided. Such behavior may be increased by increasing communication; but it is also true that the increase of co-operative social behavior often sets the problem and the means for the increase of communication. National and regional rivalries have not existed because men had no common language, but they have had no common language because they were not in fact engaged extensively enough in the kinds of social behavior which facilitated and made imperative a common language. And similarly our society is not in distress because we do not have a sufficient body of interpersonal signs, but we fail in communication precisely because we live in a society caught in a pervasive reconstruction which has not progressed far enough to have found the new symbols expressive of itself and adequate to its further development. Sign-behavior is part of behavior and develops as behavior develops; it is both controlled by and in turn exercises control over the behavior in which it appears, be this behavior individual or social.

It also needs to be stressed that the sole purpose of signs is not to prevent conflict, and that the increase of communication should not be looked upon solely in terms of making persons alike. Co-operative social behavior is possible between individuals of great diversity, and the understanding of other persons and their signs has its own rewards. Conflict amid diversity is to be condemned only when it frustrates rather than liberates. ${ }^{\nabla}$ If one of the practical tasks of the theory of signs is to further co-operative behavior, another complementary and equally insistent task is to so incite and fortify the individual that he keeps his own creative integrity in the face of the powerful forces in the modern world that tend to reduce him to a puppet pulled by the socially controlled strings of communication.

These various considerations and qualifications are not to be taken as denials of the general integrating and socializing role which communication performs in individuals and societies. The complex reaches of the self and society are dependent upon communication for their genesis and for their maintenance. The self develops by taking the role of others and incorporating in its advance the attitudes which it then assumes; and communication is a major requirement in this process. 
Modern transportation, the organization of a factory or office, the prosecution of a war, the carrying out of an election, the mutual understanding and co-operation between individuals of different cultures, in short, all forms of complex social behavior, depend upon the existence and the extension of systems of communication. While communication does not insure co-operation, it is nevertheless the primary agency for obtaining, maintaining, and extending the integration of the human individual and human society. ${ }^{\mathbf{}}$ 


\section{THE SCOPE AND IMPORT OF SEMIOTIC}

\section{Pragmatics, Semantics, and Syntactics}

WE HAVE now surveyed the range of material with which a science of signs must deal. There remains only the task of bringing to a sharper focus the scope of this science and of indicating its theoretical and practical importance.

Those readers who are familiar with the literature of semiotic may have been surprised that the terms 'pragmatics,' 'semantics,' and 'syntactics'-so widely current in this literature-have not figured in our account. Their absence up to this point has, however, been deliberate. These terms have already taken on an ambiguity which threatens to cloud rather than illumine the problems of this field, being used by some writers to designate subdivisions of semiotic itself and by others to designate kinds of signs in the object languages with which semiotic deals. Otto Neurath warned years ago that these terms would engender pseudo-problems and distract attention from genuine problems; the course of events has proved in part the legitimacy of his fears. Yet these terms, if carefully introduced, serve to mark the scope and subdivisions of semiotic, and may now be added to our terminology. In Foundations of the Theory of Signs (p. 6), the three terms in question were defined as follows: pragmatics as the study of "the relation of signs to interpreters," semantics as the study of "the relations of signs to the objects to which the signs are applicable," syntactics as the study 
of "the formal relations of signs to one another." Later analysis has shown that these definitions need refinement. Even as they stand, however, they give no warrant for their utilization as a classification of kinds of signs ("pragmatical signs," "semantical signs," "syntactical signs"); such extension of their signification is questionable, since it may blur the distinction between signs in various modes of signifying and the signs which make up pragmatics, semantics, and syntactics conceived as the three divisions of semiotic. Hence we shall not employ such an expression as "syntactical sign," since doubt can then arise whether it designates a kind of sign (say, formators) or a sign within the part of semiotic distinguished as syntactics. ${ }^{\mathbf{A}}$ The terms 'pragmatics,' 'semantics,' and 'syntactics' need clarification, however, even when restricted to differentiations of the field of semiotic.

Carnap formulates these distinctions in the following way:

If we are analyzing a language, then we are concerned, of course, with expressions. But we need not necessarily also deal with speakers and designata. Although these factors are present whenever language is used, we may abstract from one or both of them in what we intend to say about the language in question. Accordingly, we distinguish three fields of investigation of languages. If in an investigation explicit reference is made to the speaker, or, to put it in more general terms, to the user of a language, then we assign it to the field of pragmatics. (Whether in this case reference to designata is made or not makes no difference for this classification.) If we abstract from the user of the language and analyze only the expressions and their designata, we are in the field of semantics. And if, finally, we abstract from the designata also and analyze only the relations between the expressions, we are in (logical) syntax. The whole science of language, consisting of the three parts mentioned, is called semiotic. (Introduction to Semantics, p. 9.)

In terms of the approach of the present study, the indicated division of the fields of semiotic needs certain further alterations: the restriction of semiotic to a study of language must be removed, the study of the structure of languages other than the scientific must be made possible, other modes of signification than the designative must be dealt with in semantics, and this in turn requires some modification of the formulation of pragmatics. 
The following definitions retain the essential features of the prevailing classification, while freeing it from certain restrictions and ambiguities: pragmatics is that portion of semiotic which deals with the origin, uses, and effects of signs within the behavior in which they occur; semantics deals with the signification of signs in all modes of signifying; syntactics deals with combinations of signs without regard for their specific significations or their relation to the behavior in which they occur.

When so conceived, pragmatics, semantics, and syntactics are all interpretable within a behaviorally oriented semiotic, syntactics studying the ways in which signs are combined, semantics studying the signification of signs, and so the interpretant behavior without which there is no signification, pragmatics studying the origin, uses, and effects of signs within the total behavior of the interpreters of signs. The difference lies not in the presence or absence of behavior but in the sector of behavior under consideration. The full account of signs will involve all three considerations. It is legitimate and often convenient to speak of a particular semiotical investigation as falling within pragmatics, semantics, or syntactics. Nevertheless, in general it is more important to keep in mind the field of semiotic as a whole, and to bring to bear upon specific problems all that is relevant to their solution. The present study has deliberately preferred to emphasize the unity of semiotic rather than break each problem into its pragmatical, semantical, and syntactical components.

There is another current distinction which offers no difficulty in terms of this analysis: the distinction between pure and descriptive semiotic.

This distinction simply marks the difference between the formative discourse of semiotic and its designative discourse, that is, the difference between semiotic as logic and semiotic as scientific discourse. Semiotic, as a language to talk scientifically about signs, will have its own formative ascriptors (such as 'Every sign has an interpretant') and such ascriptors belong to logic; it will also consist of designative ascriptors (such as statements as to what signs signify to certain persons, how signs are combined in a specific language, the origin, uses, and effects of specific signs), and such ascriptors constitute semiotic as a natural science. This distinction applies to each of the subdivisions of semiotic: 
hence we can distinguish pure and descriptive pragmatics, pure and descriptive semantics, pure and descriptive syntactics. ${ }^{\mathbf{B}}$ The application of semiotic as an instrument may be called applied semiotic.

Does semiotic signify itself? An affirmative answer can be given to this question, without contradictions arising, provided we recognize that no sign denotes its own signification. This assertion, itself part of logic, is an analytic formative ascriptor: since the signification of a sign gives the conditions of denotation for a sign, the signification of a sign is not

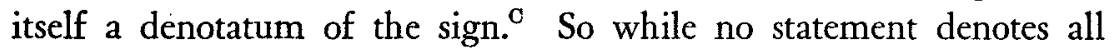
significations, there is no signification about which a statement cannot be made. In this way a statement can be made within semiotic about any sign, including the signs of this book, though no body of statements about signs is the totality of statements which can be made about signs.

\section{A Program for Linguistics}

Our discussion of signs, even of language signs, has another noticeable peculiarity: it has not been couched in the terminology current in linguistics. 'Subject,' 'object,' 'predicate,' 'noun,' 'verb,' 'word,' 'sentence,' 'modification,' 'voice,' 'phonology,' 'morphology': such terms have been deliberately avoided. This has not been done to disparage the work of the professional linguist-for certainly he of all persons has carried farthest the scientific study of language-but in order to raise in an explicit form the relation of linguistics to semiotic, and to suggest a program by which the terminology of the linguist can be grounded on the basic terms of semiotic. Only the linguist himself can carry out this program.

The justification for this program lies in the present state of linguistics itself. For decades linguists have expressed dissatisfaction with the traditional terminology in which they have talked about the spoken and written languages with which they have been concerned. And many proposals have been made for rebuilding this terminology on new foundations. The issue concerns the nature of the metalanguage in which the linguist is to talk about languages. The problem has arisen largely as the result of the study of languages other than those for which the traditional terminology was designed. This terminology appeared 
in Western linguistic science through attention to such languages as Greek and Latin within the Indo-European family of languages, and has been influenced at many points by the work of philosophers and logicians who themselves lived within this linguistic tradition. As attention widened to include languages in Asia, Africa, and America of widely diverse families, the parochial and limited character of the traditional linguistic metalanguage became obvious. The linguist found himself somewhat in the situation of early students of comparative religion who tried to describe the religions of the world in terms of one particular religious tradition. If one attempts to avoid this difficulty of talking about all languages in terms of one set of languages, or in terms of one philosophical or logical point of view, the problem then becomes acute: on what foundation is linguistics to erect its own metalanguage? This problem cannot be evaded by merely describing various languages, because a description of any language must be made in some terms or other. And any attempt to solve the problem means a reconstruction of linguistics at its foundations.

The suggestion here made is that semiotic provides the metalanguage for linguistics, and that the terminology of linguistics is to be defined by linguists on the basis of the terms of semiotic. In this way one could describe all languages of the world in a uniform terminology which would make possible a scientific comparative linguistics. A number of linguists have moved steadily in this direction, as mention of the names of Edward Sapir, Alan Gardiner, Leonard Bloomfield, and Manuel J. Andrade indicates. ${ }^{\mathrm{D}}$ And from the side of a general theory of signs, such philosophers, logicians, and psychologists as Peirce, Cassirer, Reichenbach, Carnap, and Bühler have been increasingly attentive to the material furnished by linguists. The carrying out of this program consistently and in detail would mean the emergence of a semiotically grounded science of linguistics. Since this work requires the expert training of the linguist, it does not fall within the province of this study; it is for this reason that we have not employed the current terminology of linguistics nor attempted to define this terminology in our terms nor proposed a new terminology. We must be content to indicate a program the carrying out of which will require the co-operation of the general semiotician and the specialized linguist. 
A language is completely described in terms of the signification of its simple and compound signs, the restrictions which are imposed on sign combinations, and the way the language operates in the behavior of its interpreters. These distinctions are those of semantics, syntactics, and pragmatics; hence these studies, when limited to languages, would constitute the three main divisions of linguistic science. They should in the interest of clarity replace the prevailing classifications, such as the frequent classification of linguistics into phonology and semantics, semantics being divided into grammar and lexicon, and grammar into syntax and morphology. The term 'grammar' is as we have seen especially ambiguous, covering for most linguists both syntactical and semantical considerations (usually the signification of formators but not that of lexicators). ${ }^{\mathrm{E}}$

The linguist interested in a semiotical foundation of linguistics would then employ the terms of semantics, pragmatics, and syntactics in describing and comparing languages, and where these terms are insufficient, introduce the terms he needs upon the basis of the terms of semiotic. Thus the term 'phoneme' seems to designate in a spoken language any sound which is a non-significant component of signs and yet which is influential in signification; ' $i$ ' and ' $a$ ' are two phonemes in a language if and only if there are two signs in the language which differ only in these respects and yet have different significations (as 'bit' and 'bat' in English). 'The term 'sentence' seems to coincide with 'dominant ascriptor,' the kinds of sentences corresponding to the kinds of ascriptors. The term 'word' on the other hand corresponds to no single semiotical term; so if it is to be retained it would have to be defined, presumably syntactically in terms of the degree of freedom which certain sign combinations have within ascriptors. Similarly for "parts of speech," a notion which seems to be connected with the limitation upon the mode of signifying open to certain signs in sign combinations; in a language where there are no such constant limitations on signs there would be no "parts of speech," though a given sign might signify in one ascriptor "adjectivally," in another "nominally," and so forth. In such ways linguistics would build up its terminology on a semiotical basis. What terms it will need and how they will be defined is a matter which must be left to linguists themselves. But if linguistics 
proceeds in this manner it will gain a metalanguage adequate to talk about all languages and not biased toward only one set of languages. Linguistics will also secure the advantages of a behavioral foundation freeing it from the mentalistic categories which have hindered and even now hinder its scientific advance.

My own confidence in the feasibility of this program is due to years of fruitful discussion with the linguist and anthropologist, Manuel J. Andrade. Andrade's early death prevented him from writing the book on the science of language which he had set himself as his life work. But he had himself gone a long way toward developing a semiotically grounded linguistics, much farther than any studies yet made in this field. He distinguished a number of "linguistic offices" which signs fulfilled-referential offces (under which he distinguished onomastic, deictic, and declarative), pragmatic offices, and formal offices. In terms of these offices-which are akin to the distinctions of the modes of signifying-and the limitations imposed on signs in sign combinations, he defined various classes of signs (linguistical, functional, semantical, and grammatical), and distinguished parts of speech as grammatical classes involving functional differences (that is, in our terms combinatory differences plus differences in mode of signifying). In a similar spirit he attempted to deal with all the phenomena which the linguist studies. I mention his terms, without attempting to elucidate them here, merely to show that his program involved the building of the whole of linguistics upon semiotical foundations; he believed that in this way linguistics would obtain a metalanguage appropriate to the description and comparison of all languages. It is to be hoped that some of his students will attempt to reconstruct his views and to carry further the project whose completion was prevented by his sudden death. ${ }^{\mathrm{F}}$

\section{Semiotic as Unification of Science}

The scope of semiotic has now been sufficiently explicated: semiotic is the science of signs, whether animal or human, language or nonlanguage, true or false, adequate or inadequate, healthy or pathic. There remains for consideration the question of the theoretical and 
practical importance of this discipline. Its theoretical import will be discussed in relation to the question as to the role of semiotic in the unification of knowledge, and in particular in its bearing upon the treatment of psychology, humanistic studies, and philosophy in such unification; its practical import will be discussed in relation to the problems of the orientation of the individual, social organization, and education.

As a science, semiotic shares whatever importance a science has. As it develops it will furnish increasingly reliable knowledge about signprocesses. Men have sought this knowledge in various ways in many cultures over many centuries; a scientific approach to this field but continues in this domain the development which is characteristic of every science-astronomy, chemistry, medicine, sociology, psychology, and all the rest. A scientific semiotic is at the minimum simply one more extension of scientific techniques to fields of human interest. It needs no special justification to those who have the scientific enterprise at heart.

But beyond this, semiotic has a special importance in any program for the unification (systematization) of scientific knowledge. The tendency toward the unification of science is inherent in scientific activity, since science is not content with a mere collection of statements known to be true, but aims at an organization of its knowledge. This unification occurs at two levels: on the one hand scientists seek knowledge concerning a subject matter which for various historical reasons has been broken up into separate fields studied by separate groups of investigators. Historically, for instance, the process of cell division has been studied by biologists and the process of surface tensions by physicists; today the biophysicist seeks comprehensive laws of surface tension which will apply to cell division. The other level of the unification of science is distinctively semiotical: it takes the existing language of science and seeks whatever relations can be found between the terms of the special sciences and between the laws of these sciences; this activity is essentially the descriptive semiotic of the language of science, though it may issue in suggestions for the improvement of this language. Semiotic serves in the unification of science at both levels: it provides a comprehensive language for talking about a field of phenomena (sign phenomena) which has been looked at piecemeal by various special disciplines; it provides an instrument for analysis of the relations be- 
tween all special scientific languages. It is both a phase in the unification of science and an instrument for describing and furthering the unification of science.

The sense in which semiotic is itself a phase of the unification of knowledge should be evident from all that precedes, and needs no elaboration. For our discussions have brought together material which has been approached in isolation by philosophers, logicians, linguists, estheticians, sociologists, anthropologists, psychologists, and psychiatrists, and they show, at least in principle, how this material may be organized under a common terminology within a general theory of behavior.

The role which such a science performs in the study of the relations between all other sciences needs, however, to be considered in some detail. In the unity of science movement, as represented by the International Encyclopedia of Unified Science," there are four major points at which difficulties and doubts have arisen: the relation of the "formal sciences" to the "natural sciences," the relation of psychology to the biological and physical sciences, the relation of humanistic studies to science, and the relation of philosophy to systematized knowledge. Semiotic throws considerable light on all these problems and facilitates a thoroughgoing unification of scientific knowledge.

The first of the problems-the relation of the "formal sciences" to the "natural sciences"-has already been discussed tangentially, and needs only to be restated in terms of the present context." The question is whether a unification of science can in principle eventuate in one system of terms and laws or whether an encyclopedia of unified science would stand on the shelf in two volumes, one a unification of logic and mathematics on the pattern of Russell and Whitehead's Principia Mathematica, the other a unification of the sciences of nature on the pattern of Newton's Philosophiae Naturalis Principia Mathematica.

The difficulty in a straightforward answer to this question comes in part from the ambiguity in the terms 'unification' and 'science.' The work of Russell and Whitehead shows that it is possible to bring into one system of postulates and theorems many systems of formative ascriptors which have had an independent origin and development; it does not show that all systems of mathematics are part of a single system 
which includes, say, Euclidean and non-Euclidean geometries. In the same way the work of Newton showed that statements about the motion of astronomical bodies and statements about the motions of bodies on the earth were instances of general laws of motion; it did not show that there were no mutually exclusive languages possible for physics. The unification of science then does not mean that there are no alternative languages, but that it is possible to some extent at present, and to an unknown extent in the future, to construct a single language applicable to various subject matters and allowing the formulation of general laws holding for these subject matters. Unified science is systematized science; it does not exclude the possibility of alternative systematizations.

Now in this sense of unification it seems possible to unify (systematize) mathematics and natural science, and semiotic is itself the evidence of this possibility. For semiotic provides a set of terms applicable to all signs and hence to the signs of both mathematics and natural science. This does not mean that the signs of these two domains are the same kind of signs; the distinction between formative ascriptors and designative ascriptors protects us from this error. Nor does it mean that mathematics is a natural science; mathematical discourse is distinguished in our account from scientific discourse, just as, say, poetic discourse is distinguished from scientific discourse. But it does mean that insofar as we have knowledge about mathematics this knowledge is essentially of the same kind as all scientific knowledge, resting in this case on evidence drawn from the signification of signs; hence this knowledge is in principle incorporable within a system of unified science. If we do not confuse the existence of various types of discourse with knowledge, the recognition that mathematics is not physics or poetry, and that a Euclidean geometry is not a non-Euclidean geometry, is no obstacle to the unification of knowledge. How far this unification can in fact be carried out remains an open question; semiotic, even its present stage, should give some assurance of the feasibility of the program.

So with regard to our first problem we conclude that the confusing distinction between the "formal sciences" and the "natural sciences" should be couched rather in terms of the legitimate distinction between 
mathematical discourse and scientific discourse, and that when this is done no obstacle is presented by this difference for the unification of scientific knowledge. Language has its mathematical portion and its scientific portion; the nature of each and their relations can be scientifically studied within semiotic, itself a language with a mathematical subdivision (logic or pure semiotic) and a scientific subdivision (descriptive semiotic). The fact that mathematics is used by a scientist and yet is itself not knowledge of the subject matter which the scientist studies raises no more of a problem than the fact that the microscope used by a scientist is not itself a scientific statement or a part of his scientific knowledge. And since semiotic is itself a science, its nature and its relations to other sciences can in turn be studied scientifically. Semiotic then is not only a science incorporable within unified science, but is able both to show the distinctive character of mathematics and the possibility of a single unified science which includes knowledge of mathematics along with knowledge of other subject matters.

\section{The Place of Psychology within Science}

Our second problem is whether semiotic explicates in any way the relation of psychology to the other sciences. Carnap has pointed out that the problem of the unification of science is threefold: terminologically it is the problem whether the terms of all the sciences can form one language such that the signification of the terms in all sciences can be formulated on the basis of some initial set of terms, however these are chosen; concerning the question of laws the problem is whether the laws of all the sciences can be deduced as theorems from some set of laws which serve as postulates; methodologically the question is whether all the sciences employ, in spite of differences, a common method in obtaining knowledge of their subject matters. ${ }^{\text {I }}$

The claim that psychology may in these respects be integrated with the other sciences is most frequently challenged on the ground that psychology has as its distinctive subject matter events which are private, and hence which require unique terms and laws for their description and unique methods for their study. The point to be stressed is that the admission of private events (such as emotions, after-images, ideas, and 
dreams) does not involve the consequence that psychology is not integrable into the program of unified science. The development of this point will serve to tie together the various remarks about psychology which have appeared throughout this study.

That there is a sphere of the private does not need to be questioned. Tables have characteristics which electrons do not have, and organisms have characteristics which neither electrons nor tables have. A table may be brown, weigh ten pounds, and be inflammable; an organism, in addition to characteristics similar to these, may be angry, have afterimages, feel anxious, dream, respond to events in itself and in the world as signs. These characteristics may be regarded as characteristics of the organism in the same sense as mass is a characteristic of an electron or color of a table. Further, just as the characteristics of an object may depend on its relation to other objects, so certain characteristics of an organism may depend on its relation to other organisms-the presence of anxiety, for example, or what is dreamed, or symbols which occur, may be due to the interaction of a person with other persons. Such characteristics may then be regarded as bio-social, biological in that they are characteristics of an organism, social in that they arise in social interactions. Since an organism is itself a complex interrelated set of structures and processes, its characteristics may in turn be stimuli to its own responses: a person may look at his own foot, try to relieve his anxiety, talk about his dreams.

Now when the characteristics of an organism which are stimuli to itself are not possible stimuli to other organisms, they may legitimately be called private. A pain in the toe is private in this sense, but not the toe; the dream being talked about but not the talking. But such privacy does not make the events in question other than biological or bio-social; nor does it mean that knowledge of such characteristics is limited to the organism of which they are characteristics. Let us expand these two points in turn.

It is often said that private events are "mental." But in saying this, perhaps nothing is done but to add a new term synonymous with 'private.' To call a pain in the toe 'mental' in this sense of the term is not to deny that it is a characteristic of an organism. Similarly, when an event is a sign to an individual, the disposition to respond in a certain 
way which is involved in the event being a sign may be something to which the individual alone can respond as a stimulus. In this status the interpretant in question may be called an "idea" or "thought," but the introduction of such terms need not carry the implication that the interpretant is not a characteristic of the biological organism. Hence the recognition of a sphere of the private does not warrant a general opposition of "mind" and "body" or of the "mental" and the "physical." The rapid and fruitful development of a behaviorally oriented psychology and psychiatry is evidence that such oppositions no longer constitute an obstacle to regarding the subject matter of psychology as bio-social in character. And when sign-processes are adequately brought into the account, the feeling that a bio-social psychology "leaves out" an important segment of human personality will steadily disappear.

Nor does the recognition of a sphere of the private-whether of significations or other phenomena-make impossible scientific knowledge of this sphere. For we have seen that to have scientific knowledge of something does not require that each individual be able to respond directly to the something in question, but merely that he be able to obtain evidence that statements which are made about it do denote. In some cases he may be able to confirm statements by responding to events which are private to himself (as in statements about after-images); in cases where the events are private to others he is limited to indirect evidence that the events in question occur. This evidence may be drawn from other characteristics of the organism (as in determining whether a given individual is angry by observing his behavior and physiological status) or from the verbal reports of another organism about itself. Since on our account the signification of signs is open to experimental investigation, the general accuracy of a given individual's report is subject to check, and such reports may therefore be legitimately included among the evidence used to determine whether or not a particular statement does or does not denote. It is even possible to determine experimentally the degree of interpersonality which a given sign has for a number of interpreters, and the degree to which a given interpreter is conscious or unconscious of his signs and their significations. The result is that even the sphere of the private--both with respect to 
signs and events which are not signs-is open to investigation by a behavioral psychology. The terms and laws of such a psychology can be couched in bio-social terms; and no theoretical obstacle faces an attempt to bring these terms and laws within a system embracing the terms and laws of other sciences. How far psychology will retain such terms as 'mind,' 'idea,' 'thought,' 'consciousness' is a matter for psychologists to determine. Our purpose has been merely to show that if such terms are kept they may be defined in bio-social terms provided that the psychologist has at hand a sufficiently developed science of signs. Semiotic itself does not rest upon any particular version of psychology, but a behaviorally grounded semiotic may be a powerful aid in developing a scientific psychology adequate to the full complexity of the human personality.

\section{The Humanities and Scientific Humanistics}

A third problem that is central to any program for the systematization of knowledge is presented by such humanistic material as literature, art, morality, and religion. Practically all the work of teachers and students in a "Division of the Humanities" in a university consists in talking about discourse of various kinds: about effective speech and writing, about paintings, about musical compositions, about works of literature, about systems of morality, about religious documents. Let us call such object signs (together with the activities which produce them) the humanities; let us call the metalanguage about the humanities humanistics. Once this simple distinction is made we are well on our way to resolve the problem of the relation of the "Geisteswissenschaften" to science. $^{\mathrm{J}}$

The signs of the humanities are primarily, if not exclusively, composed of types of discourse which are valuative and incitive in aimfictive, poetic, moral, religious, rhetorical, grammatical, and the like. Such modes of discourse are not scientific discourse; accordingly the humanities differ in nature from science both as regards their activities and their linguistic products. Our whole account bears witness to the importance of the humanities, and shows that their relation to science is complementary rather than antagonistic. The humanities and 
science each have their own kind of importance. Only those persons who attempt to prevent an interaction of scientific knowledge with existing appraisals and prescriptions will feel it necessary to disparage science in the name of a defense of the humanities. At the same time we are now in a position to see the dangerous onesidedness of Hume's admonition to throw all books into the fire which do not contain "statements of fact" or statements as to the relations between "ideas," for such a doctrine would in principle rule out all discourse other than the scientific. A comprehensive semiotic must agree with I. A. Richards that language is "no mere signalling system" but "the instrument of all distinctively human development." The case for science or the case for the humanities requires no disparagement of the other: they differ as types of discourse differ and they support each other insofar as the various types of discourse are necessary to the totality of human behavior.

With respect to humanistics, as the study of the humanities, it must be recognized that its language too can be of types other than the scientific. The "liberal artist" can himself be an "artist," speaking rhetorically, or poetically, or critically about his subject matter, that is, can himself use various kinds of signs for valuative and incitive ends. Much of esthetic criticism, for example, is a combination of statements and appraisals about a work of art to the end of supporting some general appraisal of the work as a whole. Such discourse conforms to the canons of adequate criticism and not to the canons of adequate science. And humanistics in this sense is only a "higher order" field of the humanities, legitimate and important though not a part of science.

But by the same token there is no obstacle to the development of a scientific humanistics. For it is possible to study scientifically all the types of discourse and the activities in which they appear. Appraisive and prescriptive signs can be talked about in terms which are not appraisive or prescriptive; the valuative and incitive adequacy of signs can be discussed without aiming to induce valuations or incite to action. So the claim frequently made by the defenders of the autonomy of the "Geisteswissenschaften" is seen to rest on an ambiguity. Humanistic studies differ from science only if they are themselves humanities; insofar as they are not they are part of science, differing from other 
sciences only in their subject matter and in the development of method appropriate to obtaining statements known to be true about that subject matter.

Humanistics, in distinction from the humanities, reveals itself to be part of descriptive semiotic, namely, the descriptive study of the types of discourse which constitute the humanities. The way is then open for a scientific experimental humanistics. Such a study would investigate the relations of the signs of literature, art, morality, and religion, to the personalities of individuals and to the forms and processes of social organization. It would study the conditions under which signs of a certain sort appeared, their communicative efficacy, the functions which such signs perform in individual and social behavior, their healthy and pathic aspects. From such a study there would result scientific knowledge of the humanities. Semiotic provides the way in which scientific method can be applied to the domains of literature, art, morality, and religion. The resulting knowledge would be science and not literature, art, morality, or religion, nor would it take the place of these forms of language or the activities in which they are embedded; a scientific humanistics is no more a substitute for the humanities than are physics and biology and sociology substitutes. But a scientific humanistics would exercise the influence on appraisals and prescriptions which all scientific knowledge exercises. It will show that many of the statements of current humanistics are excessively general and false because of their generality; that certain kinds of art and religion, for instance, are produced by and needed by some persons and not by others. The whole "theory of value," so much in the forefront of current attention is conducted in the main without factual reference to the specific valuations made by specific individuals in specific circumstances. Even a small draft of experimental material would act as a cleansing wind in such discussions. Since literature, art, morality, and religion are of such great individual and social importance, all those who are genuinely concerned for the humanities should welcome the development of the scientific humanistics which semiotic now foreshadows.

When humanistics is regarded as a part of semiotic, its incorporation into the program of unified science presents no theoretical problem. For the basic terms of a behavioral semiotic are themselves introduced 
via terms drawn from the "natural" sciences of biology and physics. If the terminology of humanistics is developed from semiotic and other sciences, then humanistics is brought within the terminological unity of the sciences. Its general methodology can be scientific; like all sciences it will develop special methods appropriate to its subject matter. And nothing prevents the formulation of laws concerning its subject matter whose relation to the laws of other branches of science can then be investigated. The humanities remain, in a distinctive sense, and their importance is undiminished; humanistics, however, emerges as a semiotically grounded science. And insofar as researches succeed in constructing a scientific language in which the terms and laws of the physical sciences and humanistics form one systematic whole, humanistics will become a part of unified science.

\section{The Language of Philosophy}

The language of the philosopher constitutes a proper object of investigation for the semiotician. To find out what "philosophy" is it is only necessary to take a number of writings commonly admitted to be philosophical and from a study of their characteristic signs determine the nature of philosophic activity in terms of its linguistic product. This study is itself peculiarly difficult, however, both because of the variety of documents which would be accepted as philosophical and the differences of opinion whether certain given works are or are not philosophical. The term 'philosophy' is then not only a vague and very general term, but over the area where it has a common signification, a term with a complex signification difficult to isolate. Semiotic offers a challenge to philosophy; it is indeed a "prolegomena to any future philosophy," demanding that philosophy make clear the nature of its signs and the purposes of its discourse. Philosophers of widely different schools today recognize the need of meeting this challenge. And there is a surprisingly large agreement, though not total agreement, that philosophy is intimately connected with semiotic; even some philosophers regard philosophy as identical with semiotic in its greatest generality. ${ }^{\mathbf{K}}$ An adequate resolution of this complex issue would be a task in itself; here we must merely open the question. 
The question may be pointed by asking the relation of the language of philosophy to the types of discourse which have been distinguished. A number of possibilities at once occur. The term 'philosophical' might not distinguish a type or types of discourse but apply-like such a term as 'witty' - to any discourse, perhaps in proportion to its generality or to its comprehensiveness; the language of philosophy might be a single type of discourse, such as the discourse called cosmological or the discourse called metaphysical; it might be identified with the language of semiotic or some portion of this language (such as with logic); finally, it might apply to a set of types of discourse, such as those dominated by the systemic use of signs in its greatest comprehensiveness.

Any one of these alternatives can be argued for in terms of historical precedent; disputes about the nature of philosophy are for the most part disputes about choices among various significations of the term 'philosophy,' choices themselves influenced by the valuation of one type of activity as more important than another. I shall choose the last of the alternatives mentioned: the language of philosophy is made up of those types of discourse dominated by the systemic use of signs in its greatest comprehensiveness.

This choice is itself defensible both in terms of historical precedence and the importance of philosophical activity when so widely conceived. For a long tradition distinguishes philosophies in terms of their cosmologies, their critiques of value, their propagation of methodologies, and with less clarity, their metaphysics as distinguished from their cosmologies (the term 'ontology' at times marks this distinction). ${ }^{\mathrm{L}}$ Our choice embodies this tradition, for the distinctions in question appear in our scheme in the four types of discourse which fall within the systemic use of signs; these four types of discourse, in their most comprehensive form, cover the traditionally recognized fields of philosophical activity and, in their totality, agree fairly well with a large body of literature commonly recognized as philosophical.

The image of the philosopher as an engine of symbolic synthesis corresponds well to the historical role of the philosopher and throws considerable light on the nature of his activity and the sources of philosophic difference. For comprehensive synthesis in the form of language symbols is certainly an identifying mark of philosophic activity in every 
culture. We expect the philosopher to speak to us in a language which makes intelligible the interrelation of all our experiences and which is relevant to our personal and social foci of tension.

Philosophic synthesis differs from other forms of symbolic synthesis in that it is addressed primarily to the critical determination of our basic beliefs. A philosophy is a comprehensive systematic organization of basic beliefs--beliefs as to the nature of the world and man, beliefs as to what is good, beliefs as to what methods should be followed to attain knowledge, beliefs as to how life should be lived. The philosopher is confronted in his culture by statements of fact, by appraisals of value, and by prescriptions for conduct. And the philosopher critically organizes these statements, appraisals, and prescriptions into a comprehensive system of beliefs.

A philosopher will have before him in symbolic form the results of the sciences, arts, and religions of the world in which he lives. Out of those portions of the material to which his own needs and experience sensitize him he will endeavor to construct a system of beliefs. To the extent that he attempts to support his basic beliefs by empirical evidence, his philosophy becomes scientific in temper; to the extent that his language is esthetic his philosophy takes on the character of art; to the extent that his basic beliefs center around a way of life his philosophy becomes religious in tone. But normally the language of the philosopher reflects all the idioms of science, art, and religion, and this comprehensiveness of mode of expression matches the comprehensiveness of the philosophic aim. However close its relationship to scicnce, art, and religion in subject matter and manner of expression, philosophy nevertheless differs from all of these in the critical comprehensiveness of the system of beliefs which it presents to us for adoption."

If this is a fair account of the nature of philosophy, it allows us to understand both why there are many philosophies and why philosophers so stubbornly resist the admission of this fact. The plurality of philosophies arises out of the differences of philosophers and the differences of the cultural material presented for synthesis at various times and places. For the philosopher is a person, and as a person his experience is not only limited but is selective. Philosophers are temperamentally more sensitive to some material than to other material, and within the 
domains to which they are sensitive their experiences differ. So some statements, some appraisals, some prescriptions attract them more than others, and the resulting systems of beliefs about "the true, the beautiful, and the good" differ from philosopher to philosopher even in the same culture at the same time. By the same token two philosophers may be similar in widely different cultures or at different times in the same culture. Philosophies differ as systems of belief differ and are similar as systems of belief are similar; this results from the fact that individuals and cultures differ and yet patterns of personality and patterns of culture recur.

Many philosophers strongly resent any such description of the nature and state of philosophy. And this is intelligible. For if a philosophy is the critical systematization of beliefs, the attainment of a philosophy is the attainment of an integration of behavior at its most basic level. To confront a philosopher with a rival philosophy challenges the entire structure of his self. So the characteristic dogmatism of most philosophies with respect to other philosophies in their culture and their neglect of the philosophies of cultures other than their own. So their tendencies to claim as knowledge what is only belief, and to confuse appraisals and prescriptions and formulizations with designative statements of fact. But, fortunately or unfortunately, each philosopher can use the same devices against his rivals. And so we are left-in spite of the dogmatic philosopher-with a plurality of philosophies. When philosophies are envisaged as systems of beliefs, this is no more nor no less than we should expect.

Nor is this result without positive significance. It does not negate philosophy, but rather opens the door to a new and more comprehensive philosophic synthesis germane to our age. For this is a time of the interpenetration of the great cultures of the world, a time of enormous proliferation of scientific knowledge, a time of basic revaluation, a time of competition between designs for living. Such an age must grope for synthesis, must welcome suggestions for synthesis from every cultural quarter. Only then can the diverse peoples of the world co-operate with mutual respect and with full justice to their diverse historical heritages. This is a time for openness of personality and not for clutch- 
ing possessively at what one already is and has. The closed and authoritarian personality structure is today being challenged by the open and flexible personality structure; this, in psychological terms, is the central struggle of our age. New types of philosophy are needed, many of them and in many forms, before a philosophical synthesis appropriate to large areas of the present world can emerge. To recognize the plurality of past philosophies, and thus to challenge the dogmatic claims of each and all of them, is itself positive support for significant philosophic work in our own time.

The recognition of a plurality of philosophies is even a step toward a new philosophy. For there is nothing in what has been said that makes impossible the attainment in long time of a philosophy which all men might share. Each of us in his own way is free to do what he can to actualize this possibility when we realize that all given philosophical systems are simply suggestions for the widest organization of human beliefs. I for one think that the philosophy toward which we are headed will be pluralistic and objective relativistic in character, and scrupulous in gathering observational evidence to support its statements, its appraisals, and its prescriptions. But whether this be true or not, and whether there be one philosophy in the far future or many, the recognition that philosophy is the symbolic systematization of beliefs cuts the authoritarian impasse which now stifles philosophic and cultural advance. It provides a bridge between philosophy and the other facets of any one culture, and between the philosophies of the great cultures which are essential material for the next stage of philosophic synthesis.

On such a conception of philosophy, philosophy is not a part of semiotic, and yet has an intimate relation to semiotic. This too accords with the tradition, represented by Aristotle, for which "logic" is an "organon" of philosophy and not a proper part of it. Semiotic (which includes logic) becomes in its expanded form the essential organon of philosophy, for if philosophy is to perform adequately its task of comprehensive systematization it needs the most adequate knowledge of signs which it can obtain. Semiotic is thus basically important material for philosophy, but philosophy is not confined to semiotic, since its discourse is not limited to scientific discourse. And semiotic in its turn is 
not a subdivision of philosophy but of science, the language of philosophy being itself but a part of the subject matter it investigates. Since the terms of semiotic apply to the language of philosophy, scientific knowledge about philosophy can be incorporated into a system of unified science.

Semiotic itself neither rests on nor necessarily implies a particular philosophy. A science of signs no more decides between an "empirical" and a "non-empirical" philosophy than it decides between a "naturalistic" and a "supernaturalistic" religion. In itself it cannot force one to believe only scientifically verified statements, nor to use only scientific discourse, nor to form one's appraisals and prescriptions in the light of science. It will nevertheless have a profound influence on the course of philosophy, since it deals with topics peculiarly relevant to philosophical systematization. Its attempt to gain scientific knowledge concerning signifying, the relation of the modes of signifying, the criteria of truth and adequacy, the nature of signs in the specialized types of discourse, and the individual and social contexts in which signs function will inevitably influence the beliefs, appraisals, and prescriptions of the philosopher. In this sense the philosophy of the future will be semiotically oriented. But the nature of this influence will not always be the same, and will depend upon the role which given individuals and societies assign to scientific knowledge.

Empirically and naturalistically minded persons will, however, find in a scientific semiotic a strong ally in the development of an empirical and naturalistic philosophy in which beliefs, appraisals, and prescriptions are formed in the light of, and held subject to control by, scientific knowledge; they will welcome a conception of philosophy which differentiates it from science and yet which completely avoids any conflict of philosophy with science. ${ }^{N}$ Individuals of another stamp will challenge a scientific semiotic or limit its relevance for the control of philosophic activity. Since the differences of philosophies reflect differences in philosophers and in cultural traditions, there will be conflicts of philosophies as long as such differences exist. And that means a long time. Semiotic will provide the battleground for future philosopher combatants; it cannot name the victor. 


\section{The Import of Semiotic for the Individual}

A scientific semiotic serves as a basis for a special form of technological discourse: discourse prescribing methods for the use of signs to accomplish various purposes. Such discourse would constitute applied semiotic and would have the same relation to semiotic that applied physics has to the science of physics. The majority of books current today which deal with semiotic are in fact treatises in applied semiotic, concerned with improvement of reading, speaking, individual and social health, educational techniques, and intercultural communication. Their appearance and their popularity is largely due to the crisis in communication inherent in any widespread dislocation and transformation of social organization. Their defects are mainly due to the current lack of a reliable body of knowledge about signs; it is hard to popularize and apply a science which does not yet exist. Our interest has been in laying the foundations for such a science, a detailed discussion of the application of the results would be the topic of another study. We shall not in these final pages attempt to formulate a "canon" for the use of signs, but will direct our remarks to some of the larger bearings of this field upon topics of central interest for the individual, for social organization, and for education.

An individual who looks at sign phenomena from the point of view of semiotic should have become more sensitive to the importance of distinguishing the various modes of signifying and the various uses to which signs may be put. Any increase in such sensitivity should enhance the individual's capacity to utilize the sign resources which culture makes available. He should be able to see the crucial importance of science in providing reliable information about himself and the world, information interesting for its own sake and a powerful factor for the formation and rectification of his appraisals and prescriptions. At the same time he should be better able to find his way around in types of discourse other than the scientific, to appropriate the resources of fiction, poetry, mythology, religion, and philosophy in determining the direction and orientation of his own life. For life as activity cannot be content with information; it needs to choose among the ob- 
jects with which it will be concerned, and it needs to develop fruitful techniques of interaction with such chosen objects. Only the individual who utilizes the signs of the artists, the prophets, and the philosophers, as well as the information given to him by the scientists, is living at the level of a complex individual. To show that signs other than scientific play a basic role in life, and yet to do this in a way which does not minimize in the least the unique importance of science, is perhaps the most important single task which semiotic can today perform.

Knowledge about signs can also serve to protect the individual against the exploitation of himself by others. From the cradle to the grave, from awakening until sleep, the contemporary individual is subjected to an unending barrage of signs through which other persons seek to advance their goals. He is told what to believe, what to approve and disapprove, what to do and not to do. If he is not alert, he becomes a veritable robot manipulated by signs, passive in his beliefs, his valuations, his activities. Through post-hypnotic suggestion an individual can be caused to perform actions suggested to him without realizing the source of his actions, and with the feeling that he is acting as a free agent. The development of the radio, the printing press, and the movies makes possible an enormous extension of influence not essentially different in kind from hypnosis. Great masses of individuals repeat each week what has been digested for their belief, buy things which they approve because they have been shown a pretty girl or a "scientist" using such articles, mechanically repeat actions which they have been assured ought to be performed. Behavior becomes stereotyped, monotonous, compulsive, and pathic. The individual loses his integrity, his spontaneity, his flexibility. Against this exploitation of individual life, semiotic can serve as a counter force. ${ }^{\circ}$ When an individual meets the signs with which he is confronted with a knowledge of how signs work, he is better able to protect himself against exploitation by others and better able to co-operate with others when co-operation is justified. If he asks himself the kind of sign he encounters, the purpose for which that sign is used, and the evidence of its truth and adequacy, his behavior shifts from automatic responses to critical and intelligent behavior in which he himself acts as a responsible and spontaneous center. He becomes an autonomous human being, neither unduly sus- 
picious nor unduly gullible, a center of life and not a hypnotized animal.

By the same token the individual can gain from semiotic some leverage against his own self-exploitation by inadequate and pathic signs. We have seen how a person may hold on to such signs inflexibly because of some partial satisfaction which they give-an imputed status which is not actual, a signified resolution of a problem which is not in fact resolved, a temporary reduction of an anxiety whose grounds are not realistically removed. Powerful mechanisms make it difficult, and at times impossible, for the individual to become aware of such signs in himself. But an understanding of how and why signs become inflexible is at least one weapon in the struggle against inflexible signs. The individual who asks himself questions about the truth and adequacy of his signs, what purposes in himself his favorite signs actually serve, and what regions of his sign vocabulary are peculiarly insensitive to exploration is at least better able to put his fingers upon his own techniques for self-exploitation and thereby to facilitate in some measure the attainment of the flexibility in his sign-processes which reflects and aids individual health.

These various considerations, taken together, may give to the individual confidence in his own production of signs. Signs are invented, tested, and controlled by individuals, and language is made by persons in their individual speech acts. Individuals differ, and their signs should reflect their uniqueness. The specialized roles which different persons play in society need special kinds of signs for their fulfillment. The types of discourse which adequately inform are no more important than the types of discourse which adequately induce preferences and actions. There is no language adequate once and for all and for all purposes. As the frontiers of life change, science must change to give the information relevant to new needs, and the technologies and the arts and the moralities and the religions must change to clarify and direct and implement the emergent aspirations. This is health in the social life of signs. Semiotic can release the individual to play his part in his own unique way in the transmission and the transformation of the sign structure upon which the complex individual and the complex society depend for their existence and for their continued growth. 


\section{The Import of Semiotic for Social Organization}

An organized human society rests on a common body of beliefs, preferences, and modes of action. Through the signs which reflect this commonage the society gains its main control over its individual members, insuring at crucial points their participation in the social behavior characteristic of the society. In crisis situations there arises a focal concern for strengthening this control over the individual so that the society may become more powerful and efficient. This process appears in its extreme form in the totalitarian regimes of the present. But in one way or another it is an issue which every society-even a democratic society-must face in the present period of social crisis. What insight can semiotic provide in this important problem?

It is hardly necessary to repeat that a theory of signs cannot itself produce the signs on which social organization must rest. Semiotic can, however, clarify the dangers and the potentialities inherent in the new agencies of communication created by modern technology, and sharpen the distinction between totalitarian and democratic social control of these agencies. For the modern world it is a crucial question how such communication agencies as the printed page, the radio, and the film are to be used-who is to control them and for what end. For they may be used for the enslavement of the individual or for the enlargement of the sphere of the individual's creative participation in society. They may be the means by which beliefs and valuations and actions of the individual are dictated "from above," or the ways by which the individual is given the material out of which he can form his own opinions, preferences, and conduct.

In actual practice the choice between these two alternative emphases is not that of either/or but one of how much. For since a society exists only insofar as there is a commonalty of purposes and commitments, any society will employ its existing agencies of communication to strengthen itself by reinforcing in its members this commonalty upon which it depends. The genuine issues concern the extent to which this is done and the way in which it is done.

As to extent, it is relevant to point out that social organization, even effective social behavior, does not require that all the beliefs, evaluations, 
and actions of the members of the society be the same. ${ }^{p}$ A certain amount of agreement is indeed necessary if the society is not to fall apart. In a democracy this segment of agreement requires at least a concern for the growth and social participation of each component member of the society, an adherence to the processes of discussion and majority vote as means by which conflicts are to be resolved, and a commitment to accept the social policies arrived at through these processes until they are changed by further application of the same processes. But such agreement on purposes and practices not only does not require the standardization of detailed beliefs, preferences, and actions, but is incompatible with such standardization. For only if the individual is allowed expression of his individuality and opportunity for exercising initiative in social participation has a society remained faithful to democratic commitments.

This complex commitment to individual initiative within a framework of social responsibility measures the difficulties and the promise of a democratic society. And it determines the direction of democratic social control of the agencies of communication. Such a society must necessarily create its own channels of communication through the use of print and radio and film. It will do this not only to further the commonage of symbols upon which it depends for its existence, but to insure that these agencies are not exclusively used by individuals or groups in ways destructive of democratic principles and methods. It will not, however, gather into its own hands exclusive control over the agencies of communication. Democratic control over such agencies is such control as will insure that these agencies, taken as a whole, further the democratic commitment with respect to the personal development and social participation of individuals. This means that the individual must have at his disposal accurate information, and a chance to consider rival appraisals and prescriptions with respect to the course of personal and social life. And it requires opposition to any control of communication which aims to restrict the individual to an automatic performance of sign-induced behavior. Only by such controls can a democratic society retain the flexibility, the diversity, and the voluntary unity which is its distinctive nature.

Semiotic can in this way offer suggestions to those whose activities 
fall in the sphere of social organization. The knowledge it will progressively develop may be used for various ends. To affect the signs of individuals is to bind them by the most powerful chains which men have devised or to place in their hands the most powerful of all instruments for individual liberation and social reconstruction. Social control of individuals through their sign-processes is inevitable, and the possibilities for such control will become ever greater as the knowledge of signs and the techniques of communication develop. The portentous question is how such control is to be exercised.

\section{Semiotic and the School}

Since a school system is simply one of the forms of social organization by which a culture perpetuates itself, the distinction between exploitative and democratic controls of the agencies of communication is of central importance for education. The totalitarian society will give no widespread attention to semiotic in its educational plans for the total population, for knowledge of sign phenomena makes it less easy to manipulate by signs those who have this knowledge. But precisely because of this fact semiotic should have a prominent place in the educational system of a democratic society.

This does not mean that semiotic as a separate discipline need be introduced into the early levels of the school system. The acquisition of skills is not facilitated by undue attention to knowledge about such skills. Skill in the use of signs would not be best served in the early years of education by the too early introduction of a technical semiotical vocabulary. But in a non-technical language, and throughout every phase of the educational system, it would be possible to acquaint students with the main kinds of signs, the purposes which they serve, the factors which hinder sign adequacy, the complementary character of the various types of discourse, and some understanding of the interrelation of knowledge, valuation, and action. The dominant aim of such instruction would be, however, the acquisition of skill in the use of signs. This would mean that the student should himself year after year be encouraged to produce signs of various kinds and for various purposes-writing and speaking poetic, moral, religious, critical, scien- 
tific, and other types of discourse. The student should not only study historical and contemporary illustrations of the various types of discourse but should actually engage in the construction and control of these specializations of language. Only in this way can he or she gain understanding translated into skill, and pass from the mere acquisition of the cultural heritage to personal participation in its continuation and reconstruction. This can be done at every stage of the educational process, using resources appropriate to the level of development of the individual. It is a task in which all teachers can have a part. But the teacher of the native language can play a central role in such education. To show what signs appear in an utterance, how they serve various ends, how they are inadequate or adequate in actual communication is at least as important as to have students label each word or sentence by terms which linguists themselves find unsatisfactory. For such a teacher semiotic provides fresh material which can serve to invigorate and correct the vocabulary of "grammar" with which he or she previously has had to be content.

At the level of higher education, a specific and detailed study of semiotic can serve to raise to fuller awareness the training in the adequate use of signs which should have occurred throughout the earlier levels. A year course might well be devoted to this topic, covering systematically the kind of material outlined in this volume. This will require new textbooks written in an appropriate style, and filled with concrete illustrative material for analysis. Textbooks adequate to this purpose do not exist, but current developments of semiotic insure their production. The available material can, however, be used, especially if the teacher will amplify it by numerous illustrations subjected to extended study and analysis. Such a course should not limit itself to information. Throughout the period of study the emphasis should still be upon the acquisition of skill by the student in the use of signs, skill in the effective formulation and communication of appraisals and prescriptions as well as statements, skill in the employment of signs in the formation of commitments and plans of action as well as beliefs. ${ }^{2}$ For only in this way can the whole person of the student be brought into the educative process and the spontaneous and responsible democratic citizen be trained. 
An education which gave due place to semiotic would destroy at its foundations the cleavage and opposition of science and the humanities. For the importance of accurate knowledge in the formation of preferences and decisions would at no place weaken the importance of forming preferences and decisions relevant to the insistent problems of personal and social life. And in work upon the common cultural heritage of its students such an education would help to recreate and transmit the common symbols required by a democratic society, while at the same time preparing the student for playing his own dynamic and constructive role in the extension of democratic processes. For in a democratic society a common language is not enough, and a rigid and inflexible language imposed from the top is not desirable. Training in the flexible use of signs means gaining the ability to enter into fruitful interaction with persons whose signs differ from one's own, "translating" their signs into one's own vocabulary and one's own signs into their vocabulary, adapting discourse to the unique problems of diverse individuals interacting in unique situations. In this way the common language of democracy would retain its flexibility without losing its integrative capacity, growing in the soil of those relations between persons where democratic processes ultimately lie and are ultimately tested. Only an education which provides for the perpetual renovation of democratic symbols can in the end insure the transmission of such symbols. And such an education will find in semiotic its natural ally.

\section{Problems and Program}

That the science of signs is both on its way and yet has very far to go is evident from all that has been said. Our account bristles with problems; it sketches a program more than it records an achievement.

On its descriptive side semiotic must make far more use than it has yet done of the data on signs which are already available. This material is widely scattered in the studies of psychologists, psychiatrists, social scientists, linguists, and estheticians. It is often in a somewhat hidden form, gathered incidentally in research which was not focused on sign phenomena. But present developments of semiotic furnish a 
language into which this data can be translated and organized, and wisdom suggests that this be done. Such data require supplementation, however, by more careful descriptive studies directed specifically to securing knowledge of sign-processes. Studies are today being carried on vigorously in the field of communication and the next years should reap a harvest. The further step is to supplement description by planned experimentation. We have indicated the need of such experimentation in the field of animal psychology, in the acquisition of language by children, in the relation of signs to the character structures of various individuals in various societies, in the mechanisms which facilitate and restrict the correction of signs, in the determination of the signification of the key symbols by which various cultures effect their co-ordination, in the varieties and extent of communication attained in the specialized types of discourse. Through careful description and deliberate experimentation semiotic will progressively widen and solidify its descriptive base.

In its logical aspect, semiotic must move toward a more precise explication and systematization of its terminology. Since sign-processes are complex interrelationships of organic and environmental events, the newer techniques of the logic of relations will be especially serviceable in the formulation of semiotic. There is no reason why a precise symbolic language should not develop, along the pattern already found in symbolic logic but applicable to the entire range of sign phenomena, and not to those typical of science and mathematics alone. In this connection the logician, as semiotician, can be expected to restate his current discussions of such topics as probability, confirmation, intensional and extensional languages, the theory of types, and the logical paradoxes in specifically semiotical terms. Logical studies may lead to simplifications and refinements of the terminology used in this book, and may even make desirable drastic changes in its whole terminological apparatus.

A terminology is not, however, a science; a science arises only when there are laws permitting predictions with respect to the material which it studies. It is essential that semiotic now seek such laws. In this task the logician and the natural scientist must co-operate. The logician interested in a science of semiotic needs to do his work in the light of the 
material offered by descriptive semiotic, studying for instance the interrelationship between the general laws of behavior and the sign phenomena described by various specialists. For insofar as the general laws of behavior permit the derivation of statements found to be true of sign-processes, predictions concerning such sign-processes are facilitated, and semiotic is brought within the sphere of systematized science. The services of the behavioral scientist are equally necessary in this task. We may properly ask the behavioristician to relate the material of the present study to whatever general principles of behavior he believes himself to possess. This will not merely suggest laws of sign-processes but will in turn serve as a check in general behavioral principles. For as we have repeatedly suggested, a behavioristics adequate to human beings can be achieved only when it has incorporated into itself the sign phenomena upon which human personality and human society depend. As the descriptive and logical aspects of semiotic expand, with mutual influence of each on the other, semiotic will become more a science and less a program.

In the meantime attempts to apply semiotic in its existing state to personal and social problems must be encouraged, not only because of the relevance of semiotic to these pressing problems, but also because in such attempts at application a science gains incentive to its growth and correction. The application of semiotic will further the growth of semiotic as a science and at the same time act as a powerful agency for personal and social health. For science both guides and is guided by practice. 


\title{
NOTES TO CHAPTERS
}

\author{
NOTES TO CHAPTER 1
}

A (p. 2) 'Semantics' is perhaps the most widely accepted name for the discipline which studies signs. 'Semiotic, the term here chosen, was used by the Stoics, John Locke, and Charles Peirce. In the medieval period the term 'scientia sermocinalis' was frequently employed. Linguists and logicians restrict 'semantics' to a part of the whole field, namely the part which deals with the significata of signs. Hence we use 'semiotic' as the general term; 'semantics' will be employed for that part of semiotic which deals with significata. Later we show how semantics, syntactics, and pragmatics are the main subdivisions of semiotic.

${ }^{\text {B }}$ (p. 3) Because of this vagueness and ambiguity a number of scientists have proposed to discontinue the use of the term 'sign.' Of course behavioristics can be developed without the use of the term; no sign is indispensable. But since the term is in such frequent usage in ordinary language and in writings of semioticians and bchavioristicians it has seemed advisable to continue the term but to use it in a more precise manner than is customary. Whether this usage is to be incorporated into behavioristics itself must be left to specialists in the field.

" (p. 5) See Karl Zener, "The Significance of Behavior Accompanying Conditioned Salivary Secretion for Theories of the Conditioned Response," American Journal of Psychology, 50, 1937, 384-403. These experiments make clear the differences in the response of the dog to situations in which the sign is present and those in which it is not present.

D (p. 8) The notion of preparatory-stimulus seems to be in harmony with Mowrer's conception of the "warning" stimuli involved in "implicit conditioning." Speaking of the augmentation of the rat's jumping to a shock when it is preceded by a sound he writes: "This augmentation of the reaction to the unconditioned stimulus, by virtue of its being preceded by a warning, or conditioned, stimulus (which does not, however, produce a gross, overt response in its own right), is here termed "implicit conditioning" ("Preparatory Set (Expectancy)-Some Methods of Measurement," Psychological Monographs, 52, 1940, p. 27). It is further related to what K. S. Lashley calls a "conditional reaction"-i.e., a reaction to something which is determined by the stimulus character of something else (see "Conditional 
Reactions in the Rat," Journal of Psychology, 6, 1938, 311-24); to K. A. William's conception of signs as stimuli to preparatory responses ("The Conditioned Reflex and the Sign Function in Learning," Psychological Review, 36, 1929, 481-97); and to what B. F. Skinner calls a "pseudo-reflex (The Behavior of Organisms, 1938). In the usage of the present account it is not necessarily implied that a preparatory-stimulus is always learned (though it may always be), nor that all preparatory-stimuli are signs. The explanation of the functioning of such stimuli is a problem for behavioristicians. C. L. Hull has suggested that they may be explained in terms of his conception of temporal patterning.

${ }^{\mathbf{E}}$ (p. 10) This notion of behavior-family is similar to, and indeed a part of, C. L. Hull's conception of the habit-family hierarchy (see Psychological Review, 41, 1934, 33 ff.). I have not attempted to probe the problems connected with the terms 'need' or 'behavior.' 'Need' is taken as roughly synonymous with 'motivating organic state' and not with 'what is necessary for survival'; so used an organism may have needs which in fact are fatal to its survival. These two common uses of 'need' must be distinguished. On the problem of defining 'need' in behavioristics see the monograph by Else Frenkel-Brunswik, "Motivation and Behavior," Genetic Psychology Monographs, 26, 1942, 121-265; S. Koch, "The Logical Character of the Motivation Concept," Psychological Review, 48, 1941, 15-38, 127-154. As for the term 'behavior,' it might be possible to identify it with 'response-sequence' or 'behavior-family.' We use 'behavior' as a narrower term than 'response' and as a wider term than 'sign-behavior.' Other uses of the term are, however, common. For a discussion of the situation see the paper by Egon Brunswik in the forthcoming monograph, Theory of Behavior (International Encyclopedia of Unified Science, vol. I, no. 10). See also the "Symposium on Psychology and Scientific Method," Brunswik, Hull, Lewin, Psychological Review, 50, 1943, 255-310.

F (p. 10) For an analysis of the term 'disposition' as applied to signs, see C. L. Stevenson, Ethics and Language, pp. 46-59.

G (p. 12) I owe the suggestion of giving only sufficient conditions for saying that something is a sign, instead of giving a definition (that is, sufficient and necessary conditions), to Alfred Tarski. This procedure seems advisable at the present state of the argument, since premature definitions may rule out other phenomena which we may later wish to include. The consequence of the present procedure is that for the moment our statements about signs are limited to signs as identified by the criteria here proposed or by any other criteria which imply these criteria.

" (p. 15) Many of the points mentioned in this section are dealt with more fully in the Appendix, "Some Contemporary Analyses of SignProcesses." 
${ }^{\mathbf{I}}$ (p. 20) For such distinctions as are made by contemporary logicians see "The Modes of Meaning," C. I. Lewis, Philosophy and Phenomenological Research, 4, 1943, 236-49; R. Carnap, Introduction to Semantics. John Dewey and Arthur F. Bentley propose a behavioral formulation of the terminology of semiotic in a series of articles beginning in the 1945 volume of the Journal of Philosophy. In previous writings (such as Foundations of the Theory of Signs), I took "mediated taking account of" as the primitive term for semiotic, defining a sign-process as a process in which something mediately took account of something else by taking account of something immediately present. The present analysis resolves this primitive term into the stimulus, response, and organic state terminology of behavioristics. Hence it provides the basis for a behavioral formulation of all terms signifying signs.

J (p. 20) There is an obvious relation between the term 'behavior-family' and the term 'sign-family': a sign-family is a set of similar events or objects which act as preparatory-stimuli for response-sequences of the same behaviorfamily. 'Thus the term 'sign-family' involves the wider concept of behaviorfamily. Egon Brunswik has used the term "cue-family" in a way somewhat similar to the present usage of 'sign-family' (see his "Psychology as a Science of Objective Relations," Philosophy of Science, 4, 1937, 233). Peirce distinguishes sign-vehicle and sign-family by the terms 'token' and 'type'; Carnap by the terms 'sign-event' and 'sign-design.'

K (p. 21) See M. Black, "Vagueness," Philosophy of Science, 4, 1937, 427-55; C. G. Hempel, "Vagueness and Logic," Ibid., 6, 1939, 163-80; and I. M. Copilowish, "Border-Line Cases, Vagueness, and Ambiguity," Ibid., 6, 1939, 181-95.

${ }^{\mathbf{L}}$ (p. 23) For a striking illustration see James K. Senior, "On Certain Relations between Chemistry and Geometry," Journal of Chem. Education, 15, $1938,464-70$.

(p. 24) Husserl, Gätschenberger, Dewey, Mead, Langer, Kecskemeti, Ogden and Richards, Pavlov, Hunter, Yerkes, Korzybski, Whetnall and many others operate with some such distinction, some contrasting 'sign' and 'symbol,' while some contrast 'signal' and 'symbol.' The bases for the distinction differ widely.

${ }^{\mathrm{N}}$ (p. 26) The example is from Jules Masserman, Behavior and Neurosis, p. 59. It might of course be the case that the light was a substitute for a more primitive sign, such as the sight of the food box used in the experiment. But since in this case the animal only obtained food when the light was on, the sight of the box was not itself a sign of food.

${ }^{\circ}$ (p. 30) See for example the paper of A. Hofstadter, "Subjective Teleology," Philosophy and Phenomenological Research, 2, 1941, 88-97. 
A (p. 32) Many writers (Allport, Bloomfield, De Laguna, Hull, Hunter, Kantor, Mead, Sapir, Tolman, Watson, Weiss, etc.) have approached language in terms of behavior. Most of them, though not all, have stressed the social nature of such behavior, and many of them have emphasized the central place which sounds produced by the organism play in the genesis of language. Few of them, however, have operated with a carefully formulated usage of 'sign' or 'language'; hence the questions that they raise are not, from the point of view of semiotic, sharply enough formulated.

${ }^{B}$ (p. 32) Social scientists generally restrict the term 'social' to reciprocal social behavior. Since however the question as to the sense in which language is social is a basic issue, we believe it wise to admit non-reciprocal social behavior.

${ }^{c}$ (p. 34) This situation makes clear that a reference to a sign as a language sign involves the consideration of situations other than the one in which a given sign-vehicle appears. If we limited attention solely to the particular situation under consideration, we could not say that the words heard by the driver were for him language signs. Any characterization of a sign as "linguistic" involves a reference to more than one situation in which the sign operates.

${ }^{D}$ (p. 34) 'Perception' is itself a very vague and ambiguous term; in some uses perception antedates sign-processes as here defined and in other uses perception itself may be regarded as a sign-process. Hence the corresponding differences between those who would make language signs always symbolic (as substitutes for perceptual signs) and those who would permit language signs which are only signals (i.e., not substitutes for other signs). Because of this situation it is not felt advisable to rest decisions as to terminology upon a doctrine of perception, nor to rule out by definition the possibility that there are both language signals and symbols. For treatments of perception see Egon Brunswik, "Distal Focussing of Perception" (Psychological Monographs, 56, 1944, 1-49); G. H. Mead, "Concerning Animal Perception". (Psychological Review, 14, 1907, 383-90); Lewis E. Hahn, A Contextualistic Theory of Perception (University of California Publications in Philosophy, 1943). It should be remarked that on our account not every responsesequence provoked by a stimulus-object is sign-behavior. A person reaching for a glass of water is not prepared to act in a certain way because of a sign, but simply is acting in a certain way to an object which is a source of 
stimulation; the fact that events (such as an image on the retina) mediate such action does not necessarily make such mediating events signs. The glass of water may of course itself become a sign; thus it might prepare response-sequences of a certain behavior-family with respect to something else (say to a person who filled the glass) and so could become a sign of a certain person's kindness. The lower limit of sign-processes is a difficult empirical problem; the semiotician, however, must avoid making all response-sequences cases of sign-behavior if he seeks to formulate a behavioral criterion for signs in terms of response-sequences.

${ }^{\mathrm{E}}$ (p. 35) This may seem too narrow a usage: cannot a person "understand" (and so share) a language without being able to speak it? Here again it is a matter of degree, and different decisions as to usage are possible. If 'understanding' merely means that one interprets a sign as do members of a linguistic community, then a person who cannot produce the signs may still be said in some sense to "belong" to the community. But if he could not himself in any sense produce some of the signs, then the signs he receives might be regarded as simply non-language signals: dogs who interpret rightly some human commands are not ordinarily regarded as members of the English linguistic community. Human beings who "understand" a language normally produce some of the signs of the language, even if they cannot do this as well or as completely as certain other individuals.

F (p. 42) The complexity of the process is of course not covered in such a statement. Material bearing on the acquisition of language by the child is found in F. H. Allport, Social Psychology, pp. 178-89; John F. Markey, The Symbolic Process, chapter 3; Neal E. Miller and John Dollard, Social Learning and Imitation, chapter 5.

G (p. 42) Mead does not deal with the grammatical aspect of language, nor devote sufficient attention to pre-linguistic signs. It may be true that the first signals of the child are gestures, but there is no reason to assume that this is true of all animals or even that all other signals acquired by a child are derivative from gestures. Further it is not easy to say whether Mead's 'significant symbol' is, when a comsign, always a comsymbol. His frequent statements that a significant symbol refers to a meaning which exists in the gestural situation prior to the appearance of the significant symbol suggests that the significant symbol is a symbol in our sense (see Mind, Self, and Society, 78 n., 79, 120-21). But the point cannot be pressed. Hence it is best to say merely that Mead's significant symbol includes our comsigns and post-language symbols. W. A. H. Gantt states that for Pavlov language "is a whole system of symbols for the more direct signals or conditioned reflexes" ("An Experimental Approach to Psychiatry," American Journal of Psychiatry, 92, 1936, pp. 1007-1021), but since on our usage not all conditioned reflexes involve signals, Pavlov's view does not 
necessarily support the position that all language signs are symbols in the sense in which we have defined the term.

${ }^{\mathbf{H}}$ (p. 43) Compare the remarks of John M. Brewster in "A Behavioristic Account of the Logical Function of Universals" (Journal of Philosophy, 33, 1936, p. 543) and Alfred S. Clayton, Emergent Mind and Education, p. 85.

I (p. 45) Contrast Mind, Self, and Society, p. 47, with pp. 160-61. P. 136 of The Philosophy of the Present seems to isolate a form of role-taking: which occurs prior to significant symbols.

J (p. 47) Mead gives as illustration the contrast between the sentinel deer whose action warns other deer of danger and the man who noticing smoke in a crowded theatre tends to call out "Fire!" "A man who calls 'fire' would be able to call out in himself the reaction he calls out in the other. Insofar as the man can take the attitude of the other-his attitude of response to fire, his sense of terror-that response to his own cry is something that makes of his conduct a mental affair, as over against the conduct of the others. But the only thing that has happened here is that what takes place externally in the herd has been imported into the conduct of the man. There is the same signal and the same tendency to respond, but the man not only can give the signal but also can arouse in himself the attitude of the terrified escape, and through calling that out he can come back upon his own tendency to call out and can check it" (Mind, Self, and Society, pp. 19091).

K (p. 50) John Dewey makes a similar distinction, though his explanation of the difference is not put in terms of concepts but in terms of language: signs are "evidence of the existence of something else" while a symbol is "a meaning carried by language in a system" (Logic: The Theory of Inquiry, pp. 51-52).

L (p. 54) This is the commonly held view. See A. L. Kroeber, "SubHuman Culture Beginnings" (Quarterly Review of Biology, 3, 1928, 325-42); J. A. Bierens de Haan, "Langue humaine; langage animal" (Scientia, 55, 1934, 40-49); Robert M. Yerkes, Chimpanzees: a Laboratory Colony. Yerkes differs from Kroeber in believing that there is some evidence of slight cultural heredity in chimpanzees, but agrees that "there is no single system of signs-vocal, gestural, or postural-which may properly be called a chimpanzee language" (op. cit., p. 51). Alfred E. Emerson links language and social heredity as giving the unique features of man: "Instead of the usual mechanism of heredity through genic patterns in the chromosomes which determines relatively stereotyped development by means of enzyme chains, the human species is the only organism which has developed a substitute mechanism for such biological heredity. ... . The development of human social heredity through learned language symbols is of such importance that this human attribute would seem to indicate the valid division line between 
the social and the biological sciences. It is what the sociologist means when he says that man is unique in the possession of a culture" ("Biological Sociology," Denison University Bulletin, Journal of the Scientific Laboratories, 36, 1941, pp. 148-49).

M (p. 55) One central problem here is whether sub-human animals have symbols (as distinguished from signals). References to symbols are common in the literature about sub-human animals, especially in discussions of delayed reactions (see the summary of the work of Nissen, Riesen, Crawford and others in R. M. Yerkes, Chimpanzee: a Laboratory Colony, chapter 10 ("Language and Symbolism"). But J. F. Markey (The Symbolic Process, p. 112) is sceptical about there being any symbolic behavior which is not social-vocal in origin; and Hull and the gestaltists give other and divergent interpretations of the delayed reaction experiments from those in terms of symbolic processes. Part of the differences lies in different uses of the term 'symbol'. All accounts agree that symbolic processes (if they occur at all) are very infrequent in animals as compared with men.

${ }^{N}$ (p. 56) There remains, of course, the open problem of whether there are any unlearned signs. If there are, Hull should have no difficulty in dealing with them. It is not unusual for students of insect behavior to speak of 'signals', but care has to be taken before asserting that such communicational devices are signs in the sense of this study-only the experts can decide this question (for some relevant data, see Alfred E. Emerson, "Communication among Termites," Fourth International Congress of Entomology, 2, 1929, pp. 722-27), and L. Verlaine, "L'instinct et l'intelligence chez les hymenopteres," Annales de la Société royale de Belgique, 58, 1927, 59-88. E. C. Tolman, in Purposive Behavior in Animals and Men, takes for granted the existence of "innate sign-gestalts". Jules Masserman, discussing the fact that cats respond to "air-blasts with marked evidences of anxiety and fear", says that "month-old kittens born in the laboratory and exposed to the blast for the first time reacted with varying degrees of fear, indicating the genetic, and possibly atavistic, as opposed to the experientially determined, nature of the response" (Behavior and Neurosis, pp. 62-63); whether the air-blast is a sign (and Masserman does not say that it is) would depend on how one treats "perception." I know of no evidence that suggests the existence of unlearned language signs. Unlearned and unmodifiable signs, if they exist at all, would in general only be useful in a fairly stable environment which would keep the signs reliable.

${ }^{\circ}$ (p. 56) A more exact formulation is given on p. 71 of Hull's Principles of Behavior. Such learning is called conditioning if a new receptor-effector connection is established, otherwise trial and error learning (p. 386).

P (p. 57) Hull himself has in later years tended to restrict 'sign' and 'symbol' to language phenomena, which he interprets as "social pure-stimulus 
acts," or to certain products of language which he calls "individual purestimulus acts." See the Appendix of the present volume for his earlier usages and for his conception of pure-stimulus act.

${ }^{Q}$ (p. 58) It would seem desirable to simplify the study of the acquisition of language by using simple artificially constructed languages; the details of the process might stand out more clearly than in the case of the acquisition of existing highly developed languages. And since sounds seem to play such an important role in the genesis of language, it would be highly interesting to make available to chimpanzees or other animals apparatus for producing sounds (or visual stimuli); this might open up a whole new field of signbehavior in sub-human animals-symbols, comsigns, and perhaps even elementary language signs. Animals may well be capable of more complex sign-behavior than has yet been observed. 


\section{NOTES TO CHAPTER 3}

${ }^{A}$ (p. 60) The problem goes back of course to ancient times (Aristotle, Philodemus, etc.), but becomes central today because of the dominant place of science in our period. All attempts to do justice to signs other than those in science are confronted with the need of distinguishing the various ways in which signs signify; hence the central position of this problem in the whole of contemporary semiotic. Almost every worker in this field is concerned with the issues involved (Ogden, Richards, Bühler, Cassirer, Urban, Stevenson, Langer, Mace, Dewey, Pollock, Sapir, Russell, Carnap, Reichenbach, Feigl, Britton, etc., etc.). It is my belief that advance on this topic has been hindered by a failure to distinguish with sufficient sharpness between the signification of signs and the various uses and effects of signs of different kinds of signification. Abraham Kaplan, in a doctoral dissertation, The Language of Value, has gone far toward making the necessary differentiations; his work has influenced on a number of points the direction of my analysis.

${ }^{\text {B }}$ (p. 68) This usage of 'expressive' is an application of Leibniz' more general usage to the case of signs. He writes: "One thing expresses another ... when there is a constant and regulated relation between what can be said of the one and of the other" (Montgomery, Leibniz Selections, p. 212). Expressiveness is not restricted to signs. And there can of course be signs of the state of an organism which are not expressive signs: thus a blush may be a signal of something about the person who blushes, but it is then simply a signal, even if the interpreter is himself the person in question, since it is not a sign furnished by the fact of production of a sign. One must be very careful not to attempt an explanation of the nature of non-scientific signs by dragging in uncritically the term 'expressive.' It seems to me that many semioticians are guilty at this point.

' (p. 73) The term 'ascriptor' is adopted from H. M. Sheffer. Sheffer, in unpublished studies, has gone far in the isolation of the distinctively significational core of designative sentences from the further relations which they have to their interpreters in being asserted, believed, etc. The distinction between designative ascriptors and "statements" will be clarified and amplified when Sheffer's work is made available.

${ }^{\mathrm{D}}$ (p. 76) The issues here are complex. C. I. Ducasse ("Some Comments on C. W. Morris's 'Foundations of the Theory of Signs'," Philosophy and Phenomenological Research, 3, 1942, 48-49) argues that the distinction 
between identifiors and designators is so basic that they should be regarded as two distinct primary modes of signifying. Thus in the case of pointing, he insists that "What is pointed to is always essentially and strictly a place; and only accidentally and elliptically a thing-viz., such a thing, if any at all, as happens to be at the place pointed to"-hence the identifior is different in kind from a sign which designates what is at a certain place and time, and so is in a different mode of signifying. I have followed Ducasse's suggestion in separating identifiors and designators but $\mathrm{I}$ am inclined to doubt whether we do in fact point to a place as such; it seems insofar as pointing is a sign at all, that it identifies the place (and time) of something or other, and to that extent involves more than location though it does not signify this more.

${ }^{\mathrm{E}}$ (p. 77) The distinction of designators from signs in other modes of signifying was not made in my Foundations of the Theory of Signs. There the significata of signs were called "designata"; in the present analysis 'designatum' has been replaced by 'significatum,' and the significata of designators are called discriminata. My earlier monograph did not adequately differentiate between signification and denotation, since it included the latter in the former. The present terminology avoids this by limiting 'signifies' to significatum; thus a sign denotes a denotatum but does not signify it. For an effective criticism of the treatment of 'designatum' in the earlier monograph, see George V. Gentry "Some Comments on Morris's 'Class' Conception of the Designatum," Journal of Philosophy, 41, 1944, 376-84.

F (p. 79) The use of the term 'appraisor' may seem at first sight to blur the important distinction which John Dewey has made between prizing something and appraising it (Theory of Valuation in International Encyclopedia of Unified Science, vol. 2, no. 4), for it might appear that the appraisor is a sign that something is liked or prized and not an appraisal of something. That this is not the case comes from the fact that an appraisor is a sign which disposes its interpreter to confer on objects a preferential status in behavior; signs which simply signify that an object is liked or preferred are not in this account appraisors but designators. Preferential behavior to objects (likings, prizings) is one aspect of behavior and need not involve signs at all. The appraisor disposes the organism to give a preferential status to something, but the organism may not in fact prefer the object in accordance with the signification. Hence it is believed that the present account preserves the distinction which Dewey is making and refines it by linking it with the specific differences in behavior which the appearance of the appraisor provokes. Dewey's own conviction that we should appraise objects in terms of their capacities to actually satisfy needs is itself a prescription about appraisors; that people do not always so appraise objects is however the justification for the present account of appraisors. Dewey is 
correct in affirming that evaluation in the full sense is more than using appraisive signs; it involves also statements about preferabilities.

G (p. 82) Irving L. Janis ("Meaning and the Study of Symbolic Behavior," Psychiatry: Journal of the Biology and the Pathology of Interpersonal Relations, p. 436) writes that "if sign-interpreters regularly take account of approval or disapproval expressed by the communicator when they perceive a given sign, valuation is included in the semantical signification of that sign." The attempt to give a signification to such signs is in the spirit of the present account; what Janis refers to, however, falls under what we have called the expressive aspect of a sign and does not isolate the distinctive feature of appraisors-namely, the way they influence the behavior of the organism in whose behavior they appear. The approval 'expressed' to others is simply, in the behavior of the organism itself, the preferential status it is disposed to accord to certain objects because of the appearance of the appraisor. It is this behavior which is distinctive and not the fact that it is 'expressed' to other observers. Further, an organism can correctly interpret the appraisive signification of a sign, and yet not agree with what is signified; all that is involved is a tendency to approval or disapproval provoked by the sign.

B (p. 82) In terms of present-day behavior theory, appraisors are perhaps one variety of the stimuli described as excitors and inhibitors. Thus Hilgard and Marquis: "A stimulus whose conditioned response has been inhibited by extinction or differentiation is called a conditioned inhibitory stimulus because of its functional properties. It does not itself elicit any observable response, but it is capable of producing an inhibition of the response to another stimulus with which it is presented" (Conditioning and Learning, p. 47). Excitors on the other hand facilitate responses to other stimuli. Since the term 'not' is an inhibitor in this sense, but not an appraisor, positive and negative appraisors are apparently only subclasses of excitors and inhibitors, namely, those excitors and inhibitors which confer a preferential status in behavior upon objects.

I (p. 83) This favored place of some response-sequences to others in a behavior-family coincides with the notion of 'hierarchy' in Hull's 'habitfamily hierarchy'; prescriptors temporarily influence the hierarchy. Hence prescriptors seem to tie in at this point with general behavior theory, just as designators are linked with stimulus-properties, and appraisors with excitory and inhibitory stimuli.

${ }^{J}$ (p. 88) 'Formata' would be called by many persons "formal" properties of signs or situations, and formators "formal" or "logical" signs. But since the terms 'formal' and 'logical' are used in so many ways, and like the term 'expressive', often give an excuse to avoid necessary analysis, they must be used with caution in the development of semiotic. The term 'syncate- 
gorematic' is objectionable because it often covers very different kinds of signs. Thus prepositions, signs of tense, etc--while having a formative component-are often predominantly identificative and designative terms and very different from the more distinctively formative signs with which they are linked under the term 'syncategorematic.'

K (p. 90) Grace DeLaguna, in her book Specch, Its Function and Development, has dealt at length with the problem of how the undifferentiated ascriptors of animals might have developed into the differentiated lanascriptors characteristic of human language. It is my belief that this account might be profitably expanded and controlled by a study of the development of language in the child. 


\section{NOTES TO CHAPTER 4}

A (p. 93). The question as to whether signs are used by an interpreter and to what end is a matter open to empirical determination, though the determination may in some cases be difficult. Whether an animal uses a stick to obtain food it cannot reach is determined by finding out whether an animal seeks food objects and persists in attempting to draw unreachable objects to it by means of a stick. Similarly, it may be discovered that a poet in various ways opposes a certain person and persistently writes depreciatory poems about this person as one way of accomplishing his aim. In both cases the goal and the use of something as means is found out by observation. If the terms 'voluntary' and 'conscious' are given a signification such that the question of their denotation is open to empirical test, then it may be shown that some uses of signs are voluntary and some involuntary, some conscious and some unconscious. The term 'function' is often employed in place of the term 'usage,' but-aside from the fact that the term has another signification in logic and mathematics-is wider than the present intended signification of 'use.' It is often said that one of the "functions" of signs is to make possible the development of the complex human personality; but signs may have this and other consequences without being used, in the present sense of the term, as means to attain these consequences. To "use" signs is to employ them as means-objects.

${ }^{\text {B }}$ (p. 94). See T. C. Pollock, The Nature of Literature; C. A. Mace, 'Representation and Expression,' Analysis 1, 1934, 33-38; H. Reichenbach, Elements of Symbolic Logic (to appear); H. Feigl, "Logical Empiricism," in Twentieth Century Philosophy (ed. D. D. Runes); C. L. Stevenson, Ethics and Language and articles in Mind, 1937 and 1938.

' (p. 97). Ogden and Richards use 'accuracy' to characterize adequacy in the "symbolization of reference," 'suitable' for the "expression of attitude to listener," "appropriate' for the "expression of attitude to referent," "judicious' for the "promotion of effects intended," and 'personal' for the "support of reference" (The Meaning of Meaning, 5th ed., p. 234). Of the terms I have employed, 'correct' seems the least suitable; we may of course simply speak of "systemic adequacy."

${ }^{D}$ (p. 99). Lying is the deliberate use of signs to misinform someone, that is, to produce in someone the belief that certain signs are true which the producer himself believes to be false. The discourse of the liar may be highly convincing. The mere making of false statements is not lying, nor 
are all forms of misrepresentation lying-as in a painting which portrays objects with characteristics which they do not in fact have. Lying is connected with the informative function, regardless of which kinds of signs are used for the purpose of misinforming.

${ }^{\mathbf{E}}$ (p. 107). This would be a generalized semiotical formulation of what is often called the "semantic" concept of truth. For an analysis and defense of this theory, see A. Tarski, "The Semantic Conception of Truth," Philosophy and Phenomenological Research, 4, 1944, 341-76. What is often called a "proposition" is the signification of a designative ascriptor, while 'sentence' is often employed for the signs which have such a signification (and the signification of other types of ascriptors as well). Designative-and otherascriptors, as a complex signification, are to be distinguished from the question of whether they are believed or not and from the question as to whether they are or are not true. Some of the relevant distinctions are discussed in other terms in C. J. Ducasse's article, "Propositions, Truth, and the Ultimate Criterion of Truth," op. cit., 317-340.

F (p. 110). R. Carnap, "The Two Concepts of Probability," Philosophy and Phenomenological Research, 5, 1945, 513-532. Since semiotic permits a sharp formulation of such differences, and an empirical study of their interrelationship, it may provide the context for a more adequate formulation of "the theory of probability." The important thing is to distinguish the question as to the reliability of signs (that is, the frequency with which signs in a given sign-family denote) from the question as to the truth of a given sign. The frequency theory defines probability in terms of sign reliability, and the degree of confirmation theory in terms of the evidence that the conditions for the denotation of a given sign are met. Whether the beliefs of animals in the presence of signs corresponds to the "probability" of the signs in question is then a matter for behavioral investigation, and not properly a part of the formal theory of probability itself.

G (p. 111). A careful and detailed elaboration of the preliminary and crude analysis in this and the following section would give a semiotically oriented epistemology. The possibility of grounding the "theory of knowledge" upon semiotic is additional evidence for the basic theoretical importance of this discipline. An adequate semiotical treatment of epistemology is yet to be achieved; the writings of Dewey, Carnap, and Reichenbach point in this direction. Their differences show that we are merely at the beginning of this development.

H (p. 114). For a discussion of this topic in terms of recent empiricist theories of meaning, see "The Limits of Meaning," Norman Dalkey, Philosophy and Phenomenological Research, 4, 1944, 401-409; "Outlines of an Empirical Theory of Meaning," A. C. Benjamin, Philosophy of Science, 3, 1936, $250-66$. 
I (p. 117). This fact, when combined with a confusion between mode of signifying and the uses to which signs can be put, may lead to the view that statements and appraisals and prescriptions have the same "meaning" and so are "interchangeable," "translatable," "identical." Thus Ray Lepley writes (Verifiability of Value, p. 78): “'This burner lights' may seem most naturally to be a factual formulation; but if it arises and functions in some contexts, say a situation in which a burner is needed quickly if an experiment is not to be spoiled, the statement 'This one burns' obviously takes on valuative import. 'This Bunsen burner is good for heating crucibles' may seem to be a valuative formulation, yet if used in an explanation to an uninitiated person or group, the statement may be 'purely' factual." From the present point of view the fact that a statement can be used valuatively and an appraisal used informatively does not make a statement an appraisal or an appraisal a statement. Since the question as to the similarity and differences in the "verifiability" of statements and appraisals demands a sharp formulation of the nature of statements and appraisals, the basic importance of a well-developed semiotic for the development of value theory is apparent. To my knowledge no theory of value has gone to fundamentals in these matters. Hence the present differences of opinion as to the relation of "judgments of fact" and "judgments of value" both in respect to their signification and their control. I believe that John Dewey's Theory of Valuation is of basic importance in distinguishing appraisals from statements and in showing how appraisals are controlled by statements. But I think that development of his ideas requires a more elaborate semiotic than he has employed.

${ }^{J}$ (p. 120). It is necessary to constantly keep in mind that a sign may be interpersonal to some degree and personal ("private" or "subjective") in other respects. Hence the fact that language signs are so dominant in human intercourse frequently obscures the fact that very little communication is often achieved in cases where both the communicator and communicatee believe otherwise. I. A. Richards' Practical Criticism illustrates this situation in the case of literature, and psychiatric material (such as the language of the schizophrenic) adds to its exemplification. A merit of the present account is that it makes it possible empirically to determine the degree to which a given sign is personal and interpersonal. The three papers by Gustav Ichheiser noted in the Bibliography help to illumine the difficulties in communication which grow out of the dynamics of interpersonal relations. 


\section{NOTES TO CHAPTER 5}

$\Delta$ (p. 123). In the years which have passed since the publication of The Meaning of Meaning, semioticians have in various ways felt the need of refining the distinction between referential and emotive types of discourse and of disentangling the various features lumped together under the term 'emotive.' But until the foundations of semiotic were better laid, so that the modes of signifying and the various uses of signs could be isolated, little basic advance in the analysis of types of discourse was possible. Writings such as Ernst Cassirer's Philosophie der symbolischen Formen and An Essay on Man, and Wilbur Urban's Language and Reality show great sensitivity to a wide range of symbolic phenomena, and so act as valuable correctives to oversimplified versions of semiotic; but their failure to deal adequately with the core problem as to the nature of sign-processes makes their work at the wider circumference more suggestive than scientific.

${ }^{B}$ (p. 123). There are, of course, other possibilities. Classification might be couched in terms of the effects of signs rather than in terms of modes of signifying or sign usage, or in terms of the levels of signs ("object language" vs. various "metalanguages"). Neither of these approaches seems, however, basic. The same is perhaps true of the attempt to isolate types of discourse in terms of the behavior in which the signs appear; it would seem that we cannot simply define religious signs as those signs which appear in religious behavior, for in religious behavior various kinds of signs appear and are used, scientific signs, for instance, as well as religious ones. Nevertheless this possibility, suggested to me by Howard Parsons, deserves further consideration. Even if not a basis for a classification of types of discourse, it is certainly relevant in the distinction, say, of religious discourse from other prescriptive-incitive language.

c (p. 124). H. E. Palmer points out ("Word Values," Psyche, 9, 1928, 1325) that the various styles of writing all contain a predominant base of "neutral" terms and differ by the relatively slight ingredient (say 5 to 10 per cent) of other terms which they contain: a few archaic terms makes the style archaic, a few colloquial words makes the style colloquial, etc. While a style is not the same as a type of discourse, his data suggest caution in characterizing types of discourse simply in terms of the quantity of the various kinds of signs employed. This does not mean that quantitative considerations are irrelevant, nor are they excluded on the mode-use classification, but it does raise the question as to the precise place they should play in 
an analysis of types of discourse. This question remains an open problem for workers in this field.

D (p. 127). On the language of science, see L. Bloomfield, Linguistic Aspects of Science (International Encyclopedia of Unified Science, vol. 1, no. 4); R. Carnap, Foundations of Logic and Mathematics (Ibid., vol. 1, no. 3).

E. (p. 131). See Félix E. Oppenheim, "Outline of a Logical Analysis of Law," Philosophy of Science, 11, 1944, 142-160.

F (p. 133). Stephen Pepper's book, World Hypotheses, isolates and compares seven of the major cosmologies. He regards these as of the same order as scientific discourse. The present view differs from that position as well as from the position that the cosmological discourse of philosophers is "meaningless" or merely "emotive" (A. J. Ayer) or superscientific in its position of a unique method for obtaining a unique kind (or even degree) of truth (W. Urban). The issues in this field are, however, still very confused, and dogmatism should be in bad repute. What is necessary is a careful distinction between science, cosmology, metaphysics, and philosophy.

G (p. 135). For Cassirer's views, see vol. 2 of Philosophie der symbolischen Formen; for Malinowski's views, Myth in Primitive Psychology, and "The Role of Myth in Life," Psyche, 6, 1925-26, 29 ff. Malinowshi elsewhere remarks that primitive peoples often do, as a matter of fact, accord to their myths a status different from their factually descriptive statements.

${ }^{\mathbf{B}}$ (p. 138). The preceding pages on poetry merely scratch the surface of the problem. It is even possible that poetry should not be regarded as a distinctive type of discourse but as a "style" which various types of discourse may assume. I. A. Richards' pioneer work on the semiotical approach to poetry (and to esthetics in general) is still of central significance; see, among other things, his Science and Poetry. As illustrations of later discussions, reference may be made to William Empson, Seven Types of Ambiguity; K. Britton, Communication, Chap. 10; Kenneth Burke, "Semantics and Poetic Meaning," Southern Review 4, 1939, 501-23 (reprinted in The Philosophy of Literary Form); John Crowe Ransom, The New Criticism. Such discussions show, among other things, the insufficiency of characterizing poetry simply in terms of "emotion" and "expression."

I (p. 139). The work of Charles L. Stevenson is especially significant for this topic (Ethics and Language). Stevenson uses the term 'ethical' in a wide sense to apply to all issues in which there is a serious questioning of aims or conduct. He also operates primarily in terms of the descriptiveemotive dichotomy of meanings. But he admits that it might be well to supplement these terms by "subdividing meaning in a number of other ways." This we have done, and proposed as a result a narrower use of the term "moral discourse." 
J (p. 142). See Abraham Kaplan, "Are Moral Judgments Assertions?" Philosophical Review, 51, 1942, 280-303. Throughout his life, John Dewey has directed attention to the prescriptive and incitive phases of sign-behavior, and their relation to scientific statements and to appraisals. This topic is most explicitly dealt with in his Theory of Valuation (International Encyclopedia of Unified Science, vol. 2, no. 4). This work is in effect a theory of criticism.

K (p. 146). The most extensive analysis of political discourse from a semiotical point of view is found in the writings of Harold D. Lasswell and his co-workers.

${ }^{\mathbf{L}}$ (p. 147). A number of students of Henry N. Wieman have approached religious discourse in terms of semiotic; there are Ph.D. theses by G. R. Bartlett, M. W. Boyer, D. E. Littlefair, and Howard Parsons on this topic at the University of Chicago. See by Wieman, "On Using Christian Words," Journal of Religion, 20, 1940, 257-69. Also E. Bevan, Symbolism and Belief. For the various appraisals of personality ideals by various religions, C. Morris, Paths of Life. Lee.

M (p. 148). See The Fine Art of Propaganda, by Alfred and Elizabeth

N (p. 151). A survey of this approach in relation to semiotic is given by Abraham Kaplan, "Content Analysis and the Theory of Signs," Philosophy of Science, 10, 1943, 230-247.

o (p. 151). One point deserves greater emphasis. Not all the terms applied to differences in discourse signify types of discourse: some of them may denote a number of types of discourse ('appraisive,' 'prescriptive,' 'philosophical') and some of them may characterize any type of discourse ('archaic,' 'colloquial,' 'witty'). Such a term as 'literature' illustrates the situation. In a wide sense it would apply to any written document; in a narrower sense it applies to any written discourse whose style of utterance is itself approved, regardless of mode of signifying or subject matter; in a still narrower sense it denotes a number of types of discourse (poetic and fictive primarily, perhaps mythical). T. C. Pollock's The Nature of Literature seeks to isolate the characteristics of "Literature" as a specific type of discourse. Literature is held to consist of "concrete" signs which, unlike the "abstract" signs of science, express, communicate, and evoke a "full" experience and not merely an element of such an experience. Literature is conceived as a form of evocative symbolism and science as a form of referential symbolism. The account, while suggestive, seems to me to bog down through failure to clarify such terms as 'express,' 'communicate,' 'evoke,' 'full experience'; when analyzed I think it is found that these terms give no differentiation of 'Literature' as a special type of discourse.

${ }^{P}$ (p. 152). Valerie Saiving has called my attention to the fact that the 
treatment of the four systemic uses of language in this chapter is more restricted than it should be in terms of the mode-use approach. Thus designative-systemic discourse is limited to designative systematization of designative ascriptors; she argues that it should include the designative systematization of any kinds of signs whatsoever. By the same argument the restrictions made in regard to appraisive-systemic and prescriptive-systemic discourse would be lifted. The point seems to me to be well taken. The consequence is that I have illustrated in each case only one of the four possibilities for each of the four forms of systemic discourse. Since there are many subvarieties of every type of discourse, this may seem unimportant. But since the subvarieties in this case are called for by our terminology itself, the matter deserves further exploration. Such exploration might deepen, for instance, the treatment of critical and propagandistic discourse by uncovering important varieties not illustrated in our account. 


\section{NOTES TO CHAPTER 6}

A (p. 154). Many semioticians (such as Mead, Ogden, Richards) virtually ignore formators; a number of symbolic logicians have held variations of the view that "logical signs" are "auxiliary devices" which influence signification but do not signify; philosophers have often tried to regard formators as lexicatively designative of the non-linguistic situation; linguists (Andrade, Bühler) have at times made them lexicators signifying language; Russell has interpreted formators as expressive of a state of the user ('or' expressing "hesitation"); Peirce, Ducasse, and others have at times suggested that formators are prescriptive signs, commanding the interpreter of the sign combinations to use the given signs in a specific way. For various treatments of the nature of formators see also the writings of Wittgenstein, Eaton, Britton, Dewey, Carnap, Quine, Tarski, Reichenbach, Gardiner, and Bloomfield, listed in the bibliography. Historically the problem of the nature of formators was approached in the discussion of the relation of syncategorematic to categorematic terms; today it is often formulated as the relation of "logical" signs (or "formal" signs) to "descriptive" signs. Carnap admits that the logician has not given (at least at present) a general criterion for the distinction between these two kinds of signs (Introduction to Semantics, pp. 56, 59). The distinctive feature of the present account lies in its attempt to state the problem and its resolution in behavioral categories.

${ }^{B}$ (p. 157). The formulation of the signification of a formator provides then one criterion of whether a sign is a formator: if in the formulation of the signification of a sign there is a reference to the way something is signified by other signs in the particular sign combination in which the sign in question appears, then the sign in question is a formator. The fact that the formulation involves the designation of other signs, does not of course mean that the formator designates such signs. The frequent formulation of the signification of formators in terms of "truth tables" (that is, " $p$ or $q$ ' is true if and only if at least one of the two, 'p,' ' $q$,' is true") obscures this, since it suggests that 'or' designates a relation between the truth of sentences; it thus confuses the 'or' in 'p or q' with the 'or' in 'or ('p,' ' $q$ ')'-the first 'or' being a formator, the second a designator (signifying sets of ascriptors in which at least one ascriptor is true). The two are therefore different, though closely related signs.

' (p. 159). 'No,' 'not,' and 'is not' are often confused, even by logicians. 
'No' and 'is not' are formators, 'no' being a determinor, 'is not' being a connector analogous to 'is'; 'not all' is a determinor. 'Not,' if it ever occurs in the sense of 'false,' would be a lexicator signifying a property of ascriptors, and not a formator.

${ }^{D}$ (p. 161). To call a sign formative does not mean that in some occurrences of the sign it may not be lexicative or even that in a given occurrence it may not be both formative and lexicative. Numerical expressions may be a case in point. The fact that they are defined in Principia Mathematica in terms of "logical signs" shows the possibility of the formative analysis, while the view which regards them as signifying "properties of properties" may justify the lexicative analysis (thus when it is said that there are "two chairs" in the room, that is, that the set of chairs in a given room can be put into a one-to-one correspondence with the set of covers of this book, something is lexicatively signified about the chairs). A similar situation is encountered in variables. 'The X's and y's and p's and q's of formulas may be ambiguous lexicators (signifying whatever is signified by the members of a certain set of signs) and yet as establishing relations between signs in a sign combination (which signs are the same in signification and which different) they may be formators of the type to be called connectors. Nothing in the pages which follow depends on an answer as to the sign status of numerical terms or variables.

E (p. 162). Another possibility, reducible to the second or independent of it, depending on the interpretation of variables, would be to regard a formative ascriptor as any ascriptor in which all lexicative signs have been replaced by variables (as 'Some $\mathrm{x}$ is $\mathrm{y}$ ' or 'Some ... is ... .'). But the sign combinations which result in this way can hardly be regarded as ascriptors: they are rather ascriptor forms or ascriptor matrices, that is, the forms of ascriptors rather than ascriptors. Such ascriptor forms are important and are dealt with in linguistics and logic, but they do not provide the differentiation of formative discourse which we are endeavoring to isolate. It should be pointed out that further study is necessary as to the relation of formators to formative ascriptors. It may be possible to start with the latter and to isolate formators as those terms for which a variable cannot be substituted in a formative ascriptor without causing the latter to lose its status as a formative ascriptor. This would bring the present account into close relation to the positions of Quine and Reichenbach.

F' (p. 164). It is a central task of this chapter to suggest how the work of the symbolic logicians, and of Carnap in particular, can be brought within a behaviorally grounded semiotic. Yet many such logicians will find the present definition of formative ascriptors objectionable, for they do not in general introduce any qualification concerning denotation into the criterion of formative ascriptors. It is believed, however, that they do in effect make 
such a qualification implicitly in the usual assumption that identifiors by their very nature denote (an assumption we have called in question) or in the assumption of Principia Mathematica that there is at least one individual. It seems to me, however, that if we are really to lay the ghost of the "synthetic a priori," we should avoid a construction of semiotic in which denotation is ever involved in signification, and this requires in turn the qualification here given with respect to denotation in defining formative ascriptors. I might also call attention to the point that one could regard formative ascriptors as a special limiting case of lexicative ascriptors depending on what kinds of lexicators occur in them, that is, as designative ascriptors with null designative signification, etc.; Wittgenstein's and Carnap's analyses in terms of "null range" might suggest this conception. I think it is less confusing, however, to say that ascriptors which as a whole have null lexicative signification (even though lexicators occur in them) are not lexicative but formative. This still permits them to signify situations formatively (though not lexicatively), to denote, and to be true or false.

${ }^{\mathrm{a}}$ (p. 167). This confusion seems to me to be involved in the frequent attempts to replace a two-valued logic (in which every ascriptor is true or false) by multivalued logics (in which each ascriptor has more than two possible values). The multivalued systems can perhaps be regarded as a classification of ascriptors as known, and here of course more than two classes are possible (thus we might have ascriptors known to be true, known to be false, not known to be true or false). And any number of classes can be obtained if the classification be based on reliability. But when this is done the multivalued system in no sense replaces the dichotomy of true and false ascriptors. The same point comes out in the "intuitionist's" demand that we have evidence that a certain entity can be "constructed" before we admit the entity into mathematics. For a theorem, for example, may be an analytic formative ascriptor in a language without us knowing whether it is such or not. To argue otherwise is to confuse the nature of a formative ascriptor with our knowledge about formative ascriptors. Carnap's statement that "a sentence of a semantical system S [is] (logically true or) L-true if it is true in such a way that the semantical rules of $S$ suffice for establishing its truth" (Foundations of Logic and Mathematics, p. 12) might suggest the confusions we have pointed out, but in the technical development of his views (as in his Introduction to Semantics), it is clear that he has avoided the confusion in question: his "L-true sentence" is the same as our analytic formative ascriptor; while for him if a sentence is L-true it is true, this is not a definition of 'L-true' in terms of 'true'; and he admits that a sentence which states that we know that a sentence is true, is synthetic and not a formative ascriptor. 
II (p. 170). This situation perhaps explains the position of Wittgenstein in the Tractatus Logico-Philosophicus that there are sentences which "show" something about a language without talking about (that is, designating) the language. It should be stressed that the text at this point leaves unanswered the question whether all formative-informative discourse is to be called logico-mathematical or only some portion of it; and also whether "logical" and "mathematical" portions of logico-mathematical discourse are to be distinguished, and if so, how. The latter distinction has been variously sought in terms of the complexity of formative ascriptors, in terms of the kinds of formators which appear, and in terms of the formative ascriptors inherent in the language as opposed to those which appear in the language if certain lexicative ascriptors are admitted.

I (p. 171). Thinkers with emphases as diverse as Carnap and Dewey agree on the roles which logico-mathematical discourse plays in the organization and verification of lexicative ascriptors. See Carnap, Foundations of Logic and Mathematics, and Dewey, Logic, Chapter XX, "Mathematical Discourse." H. Hahn's pamphlet, Logik, Mathematik und Naturerkennen, is especially suggestive on this topic.

J (p. 176). For a discussion of the relation of metaphysics to semiotic see W. A. Wick, Metaphysics and the New Logic.

${ }^{\mathrm{K}}$ (p. 181). The next section will show how this definition might need further restrictions if other disciplines (such as rhetoric and grammatic) are considered part of the formative discourse of semiotic. If they are not so considered, no restriction is necessary.

${ }^{\mathrm{L}}$ (p. 182). On the relation of logic and mathematics see Carnap's Foundations of Logic and Mathematics and Peirce's Collected Papers. It should be noted that not all thinkers agree that mathematics is non-designative; thus E. Cassirer holds that mathematics does not, as Hilbert maintains, have signs for its objects, but signifies the structure of the world (Philosophie der symbolischen Formen, III, part 3, chap. 4, "Der Gegenstand der Mathematik"). One of the advantages of our analysis is that it does not restrict the alternatives for mathematics to either designating the world or designating signs, for formative-informative discourse does neither. While semiotic is felt to be the place in which to discuss the foundations of mathematics and its relation to logic, it is not maintained that mathematics is part of semiotic. In this respect our position is similar to Peirce, though Peirce is not consistent as to whether logic as semiotical is formative discourse or scientific discourse; he at times identifies logic with "the general necessary laws of signs" (Collected Papers II, \$93), and at times makes it a "science of fact" (ibid., I, p. 112) which even includes "speculative rhetoric" or ("the theory of inquiry"). It may be remarked that Aristotle grounded both the prin. 
ciple of contradiction and the principle of excluded middle on the nature of significative discourse (Metaphysics, $\Gamma 4$ and 7 ).

M (p. 182). This is regarded as one of the important results of our study. It shows in outline how a behavioral semiotic can incorporate both experimental studies and the work of contemporary symbolic (or analytic) logicians. Such work has rested on the distinction of logical and descriptive signs and on the distinction between logically determinate and logically indeterminate sentences. In so far as we have indicated the behavioral base of such distinctions, the way is open to define all the terms of logic on the basis of the terms of semiotic. Carnap has gone far in this respect (see his Introduction to Semantics and his paper "On Inductive Logic," Philosophy of Science, 12, 1945, 72-97). It is only necessary to define his primitive terms in ours in order to incorporate his studies into semiotic. (For instance, he regards the term 'range' as a sufficient basis for "logical semantics" (Introduction to Semantics, $\$ 20$ ); I believe it can be shown that 'range' is simply the formulation of the signification of a sign, and that his notion of "statedescription" in terms of which he defines 'range,' implicitly involves the condition of denotation which we have explicitly incorporated into our account of formative ascriptors). In so far as the terms of logic are defined semiotically we will be in a position to carry out in detail the incorporation of logic ("inductive" as well as "deductive") within semiotic which was suggested in the classical world, developed by the Scholastics, championed by numerous thinkers of the Enlightenment, and brought to a focus in the contemporary world by the work of Charles Peirce.

N (p. 182). John Dewey, for instance, in his Logic: The Theory of Inquiry argues for the interpretation of logic as the "theory of inquiry," a discipline which would be similar to what Peirce regarded as "speculative rhetoric" and included within logic. An "inquiry into inquiry" may however be stated in terms of various modes of discourse: it might consist of statements about how inquiry has been carried on (scientific discourse), or it might prescribe methods of inquiry in terms of their adequacy to reach certain goals (technological discourse) or it might prescribe the methods for inquiry (propagandistic discourse) or it might aim to influence the preference for some methods rather than others (rhetorical discourse). Now all such ways of signifying inquiry are important, and their identification (in whole or in part) with logic can summon historical precedent to its defense. To choose another signification for the term 'logic' is not to oppose Dewey's contentions that formative discourse (whether mathematics or logic as we have used the terms) is to be explained behaviorally, that such discourse performs (as does all discourse) certain important and specifiable functions within the process of inquiry, and that inquiry itself occurs in the context 
of adaptive behavior. C. I. Lewis has, in various writings, made a similar point. I take it that the present account of formative discourse, while developed so as to assimilate the work of the "formal" logicians to semiotic, does this in a way compatible with the framework of Dewey's thought. 


\section{NOTES TO CHAPTER 7}

A (p. 190). The strength of C. S. Peirce's version of semiotic lies in part in its ability to handle non-vocal signs, since it does not restrict signs to any specific medium. Peirce himself gave the iconic sign considerable attention. Since his basic interest was in scientific and logico-mathematical discourse, his treatment of appraisive and prescriptive modes of signifying and the use of signs for other than informative purposes is insufficiently developed. Students of psychology and the socio-humanistic studies have forced this development.

${ }^{B}$ (p. 191). Ernst Cassirer has stressed this point. G. H. Mead argues that the perception of objects as enduring is not possible without language ("Concerning Animal Perception," Psychological Review, 14, 1907, 383-90). For material on social perception, see F. Heider, "Social Perception and Phenomenal Causality," Psychological Review, 51, 1944, 358-74.

c (p. 191). Edward Sapir contended that some vowels had designative signification ("A Study in Phonetic Symbolism," Journal of Experimental Psychology, 12, 1929, 225-39).

D (p. 193). Cf. John Dewey's remark that "probably a time will come when it will be universally recognized that the differences between coherent logical schemes and artistic structures in poetry, music and the plastics are technical and specialized, rather than deep-seated," (Philosophy and Civilization, pp. 120-121).

E (p. 195). In my article "Esthetics and the Theory of Signs," Journal of Unified Science, 8, 1939, 131-150, I tried to differentiate the esthetic sign as an iconic appraisor. The present position is more general, since the approach to the arts in terms of the valuative use of signs does not require that the signs in art be iconic or in the appraisive mode of signifying. Iconic appraisive signs are nevertheless of great importance in the arts, and the article mentioned is still relevant to the general problem of art considered as a sign phenomena. It was discussed by the literary critics, Allen Tate (Reason in Madness) and John Crowe Ransom (The New Criticism). For supplementations as to the relation of the arts to signs see G. Kepes, Language of Vision, and Ernst Kris "Approaches to Art" (in Psychoanalysis Today). Kepes aims to show that modern painting is a discovery of new ways of representation; Kris (as does Dewey) interprets art as a form of communication. 
F (p. 196). The emphasis on the relation of language signs and postlanguage symbols to thinking was a central point of John B. Watson's version of behaviorism. It is continued in the newer versions of $\mathrm{E}$. C. Tolman and Clark Hull. Karl Lashley, one of the two original behaviorists, has in recent years expressed serious doubts as to the general thesis.

" (p. 197). See on the general question of freedom my article "The Mechanism of Freedom" in Freedom, Its Meaning (ed. Ruth Anshen); also G. H. Mead The Philosophy of the Act. A semiotical formulation of the term 'freedom' overcomes the opposition of freedom and causation by phrasing the problem in terms of the extent to which an organism causes its own behavior through the operation of signs. One who takes the position of the present volume is not surprised, for instance, that a person can cause the dilation of the pupils of his eyes by producing language or post-language signs which signify the objects which originally caused this dilation. (See the article by C. V. Hudgins, "Conditioning and the Voluntary Control of the Pupillary Light Reflex," Journal of General Psychology, 8, 1933, 3-51.)

${ }^{H}$ (p. 198). Hypnosis (and certain forms of propaganda) are of interest in this connection: they seem to cut down the intervening sign-processes of which the individual is capable, and thus allow the interpretants of the signs with which the individual is confronted to take overt form more directly and quickly-laughing when something is signified as funny, crying when something is signified as unfortunate, responding to commands in an almost automatic fashion. Such phenomena are, incidentally, confirmations of the view that the interpretant of a sign is a disposition to respond in certain ways. On hypnosis and sign phenomena, see Clark L. Hull's Hypnosis and Suggestibility, especially the final chapter.

1 (p. 198). George K. Link, "The Role of Genetics in Etiological Pathology," The Quarterly Review of Biology, 8, 1932, 133, 138.

J (p. 199). See his Behavior and Neurosis, and Principles of Dynamic Psychiatry. Cf. W. Horsley Gantt, Experimental Basis for Neurotic Behavior (Psychosomatic Medicine Monographs, 1944). These books, and the articles of $\mathrm{O}$. H. Mowrer, indicate that a behavioral development of psychiatry is possible and is indeed appearing.

K (p. 200). See O. H. Mowrer, “A Stimulus-Response Analysis of Anxiety and Its Role as a Reinforcing Agent," Psychological Review, 46, 1939, 553-65. Mowrer stresses the important point that a person may be motivated not only by needs but by the anticipation of needs. For him all anticipation involves anxiety. So signs may not only produce anxiety and motivate behavior, but they may serve to reduce anxiety-a fact which throws much light upon magic, superstition, certain forms of religion, and neurotic symptoms. See R. R. Willoughby, "Magic and Cognate Phenomena: An Hy- 
pothesis," in A Handbook of Social Psychology, pp. 461-519. Mowrer's approach constructs, via signs, a bridge between the psychiatric phenomena of anxiety and Hull's version of behavioristics.

${ }^{\mathbf{L}}$ (p. 201). Ernest Jones says of the symbol, in the Freudian sense of the term: "the individual has no notion of its meaning, and rejects often with repugnance, the interpretation" offered of it ("The Theory of Symbolism," British Journal of Psychology, 9, 1917-19, 184). I believe that symbols in this sense are signs as we have used the term, and that they can be brought within our behavioral account. To say that someone has a symbol and yet "has no notion of its meaning" is possible if a number of distinctions are made. Leaving aside cases where something is not a sign to the individual who produces it but only an expressive sign to someone else who interprets it, it may be the case that the individual for whom something is a sign may not know that it is a sign, may not signify the sign to be a sign, and may not be able to formulate its signification. In such cases it is intelligible to say that the sign has a meaning, but that the individual does not "know" what it is, and such phrases as "unconscious sign" or "unconscious meaning" or "unconscious mental process" may be most charitably interpreted in such terms. What the Freudian has done is to propose a theory as to why the individual finds it difficult to formulate the signification of certain of his signs and indeed why he actively resists such a formulation by himself or others. This theory, in so far as it is sound, is a contribution to the understanding of pathic signs; there is nothing in it which in principle cannot be translated into the terminology of a behavioral semiotic. The Freudian symbols are essentially general icons and as such capable of denoting objects which are like themselves in certain respects only (dreams of flying as symbolic of the erect penis, dreams of open books as symbolic of the female genitalia, etc.); they are a special case of metaphorical signs, namely, where certain processes in the individual prevent or make difficult the recognition that the metaphorical signification provides partial satisfaction of a frustrated desire. For the psychoanalytic discussion of such symbols see the above article by Jones; the relevant chapters in Freud's Interpretation of Dreams, Introductory Lectures on Psychoanalysis, and New Introductory Lectures on Psychoanalysis; and an article by A. A. Brill, "The Universality of Symbols," The Psychoanalytic Review, 30, 1943, 1-18.

I (p. 202). This, of course, should be accompanied by a study of the differences in sign-behavior of individuals of different physique, temperament, and character. The work of W. H. Sheldon provides a basis for such a study (see especially his The Varieties of Temperament). A preliminary investigation from this point of view has been made by Fillmore H. Sanford, "Speech and Personality: A Cooperative Study" (Character and Personality, 10, 1942, 169-98), in which he draws upon his doctoral dissertation 
"Individual Differences in the Mode of Verbal Expression" (Widener Library, Harvard, 1941). See also the papers by J. Krechel, E. Sapir, E. R. Balkan, and J. H. Masserman in the bibliography of the present book.

N (p. 202). Behavior and Neurosis, p. 8. I am indebted to Dr. Masserman for many conversations on the pathic functioning of signs, and also to talks with Harry Stack Sullivan, Erich Fromm, and Ernst Kris; suggestions and examples from these persons are used in my discussion of this topic. Dr. Sullivan has written that "psychiatry ... rests upon the study of the origin, growth, and manifestations of symbols" ("The Importance of a Study of Symbols in Psychiatry," Psyche, 7, 1926-27, p. 81); his emphasis upon signs and their relation to mentality was preceded in this country by Adolf Meyer, W. A. White, and S. E. Jeliffe, and in England by Henry Head. Reference should also be made to Kurt Goldstein's article, "L'analyse de l'aphasie et l'étude de l'essence du langage," (Journal de psychologie, 30, 1933, 430-96); and to Language and Thought in Schizophrenia (J. H. Kasanin, editor).

${ }^{\circ}$ (p. 203). It is such tendencies, segments of response-sequences, that seem to be involved in compulsive behavior and in symptomatic acts and autistic gestures. For data on such behavior see T. M. French, "An Analysis of the Goal Concept Based upon Study of Reactions to Frustration" (The Psychoanalytic Review, 28, 1941, 61-71) and M. H. Krout's article, "Autistic Gestures," Psychological Monographs, 46, 1934-5, 1-126.

${ }^{P}$ (p. 204). Psychoanalytic therapy has been largely limited to verbal techniques, allowing the individual to become aware of the signification of his signs and in this process to reconstruct his own signs and behavior. The present tendency to link psychoanalysis with medicine and the social sciences represents a corresponding merging of the techniques of therapy (the use of drugs, for instance, and deliberate attempts to change the social environment in which the individual acts). In this connection see the paper by Jules Masserman, "Language, Behavior and Dynamic Psychiatry" (International Journal of Psychoanalysis, 25, 1944, 1-8).

Q (p. 205). The words of B. Malinowski are in his article "Culture" in the Encyclopedia of the Social Sciences; Dewey's statements are in his Logic, pp. 46, and 20. For a recent discussion of the term 'culture' by an anthropologist see C. Kluckholm and W. H. Kelly, "The Concept of Culture," in The Science of Man in the World Crisis (Ralph Linton, editor).

${ }^{\mathbf{R}}$ (p. 207). See especially his paper "The Genesis of the Self and Social Control" (International Journal of Ethics, 35, 1925, 251-77). The works of Jean Piaget (The Language and Thought of the Child, and Judgment and Reasoning in the Child) seem to me to confirm the direction of Mead's analysis.

" (p. 209). Margaret Mead, in her chapter on "The Deviant" in Sex and Temperament in Three Primitive Societies, states her conviction that the 
deviant is not always to be explained by early defective conditioning: in some cases there is "a fundamental discrepancy between his innate disposition and his society's standard."

T (p. 212). "Reflections on Communication and Culture," American lournal of Sociology, 44, 1938, 187-205. Park holds that collective action requires communication, and that institutions, which distinguish human and animal societies, are due to communication. K. Mannheim's discussion, in Part III of Man and Society, of crisis, war, and dictatorship, provides good examples of substitute goals and gratifications in social processes.

$\checkmark$ (p. 215). See Karen Horney, Self-Analysis, p. 303.

$\checkmark$ (p. 216). Milton B. Singer has, on reading this chapter, called my attention to the ways in which a general theory of signs is involved in, and needed for, very practical social problems. The Supreme Court in 1915 upheld the validity of motion picture censorship by states on the ground that motion pictures were a form of entertainment and not a means of communicating ideas; in the case of Thornhill v. Alabama (310 U.S. 88) the Supreme Court in 1940 defended peaceful picketing as a necessary means of communication and hence as falling under the protection of freedom of speech; in the 1942 discussions as to whether a state could compel the salute to the flag by children in the public schools both sides of the argument appealed to specific theories as to the nature of symbols (see Official Reports of the Supreme Court, Preliminary Print, vol. 319 U.S., Number 4, pp. 632-3, 662). 


\section{NOTES TO CHAPTER 8}

${ }^{\Delta}$ (p. 218). A. Kaplan and H. Reichenbach have, among others, used such terms as 'pragmatical sign.' This extension, which I regard as unwise, is perhaps in part due to my Foundations of the Theory of Signs. For in this work a distinction was made between the pragmatical, semantical, and syntactical "dimensions" of sign-processes themselves; this, coupled with the fact that that work did not sufficiently distinguish the modes of signifying nor the difference between the signification and use of signs, might suggest such expressions as "pragmatical sign" and "syntactical sign"-expressions which the present analysis avoids.

${ }^{B}$ (p. 220). R. Carnap makes the distinctions in the case of syntactics and semantics but not with respect to pragmatics; thus on p. 6 of his Foundations of Logic and Mathematics he writes that "pragmatics is an empirical discipline." But in pragmatics too we can distinguish between formative and lexicative ascriptors, and so generalize the distinction between pure and descriptive semiotic. The present volume neglects in the main the important work which has been done in syntactics; see Carnap's Logical Syntax of Language, and The Formalization of Logic. On our position, any kind of discourse can be studied syntactically. Hence syntactics includes Carnap's "logical syntax" as the pure syntactics of the language of science. By the same token any type of discourse (such as mathematical discourse) can be studied semantically.

${ }^{\mathrm{C}}$ (p. 220). A sign itself, as a sign-vehicle, can be denoted by itself, as in the statement, 'This sentence is composed of seven words.' The recognition of this, together with the recognition that a sign cannot denote its signification, gives, it is believed, the results desired by Bertrand Russell in framing the simple theory of types, without making this theory an arbitrary principle and without making it impossible for a statement to denote all statements as sign-vehicles.

${ }^{D}$ (p. 221). E. Sapir interprets linguistic facts as "specialized forms of symbolic behavior" ("The Status of Linguistics as a Science," Language, 5, 1929, p. 211); A. Gardiner writes that the interest of the student of linguistic theory "is, in fact, what has been variously called semasiology, significs, or semantics. It is a wide field, and when rightly understood, embraces the entire domain of both grammar and lexicography" (The Theory of Speech and Language, p. 85); L. Bloomfield puts linguistics within the general theory of signs in his monograph Linguistic Aspects of Science (International 
Encyclopedia of Unified Science, vol. 1, no. 4); M. J. Andrade's views are mentioned in the text. Important and suggestive papers by European scholars, semiotical in orientation, are found in Acta linguistica and Travaux du cercle linguistique de Prague. Attention may be called to the semiotical orientation of the forthcoming book of Roman Jakobson, Sound and Meaning, to appear in 1946 as the first issue of Publications of the Linguistic Circle of New York.

${ }^{E}$ (p. 222). I have with reluctance omitted from the text an attempt to translate the key terminology of Leonard Bloomfield's Language into the terminology here used. To do this proved too detailed a task for the purposes of this study. I am convinced, however, that this can be done, and that Bloomfield's careful work offers one of the best aids for carrying out the linguistic program here indicated, for his own orientation is itself to some degree semiotical. His book is also evidence for the statement that "grammar," as usually developed by the linguist, is both semantical and syntactical in nature. A classification of parts of speech can with profit utilize Carnap's concept of isogenous. Two signs are isogenous if whenever an ascriptor which includes one remains an ascriptor when the other is substituted. A class of signs isogenous with each other but not with signs outside the class constitutes a sign genus. Thus through the notion of isogenous we can attain a classification of linguistic signs into mutually exclusive classes.

F (p. 223). Since Andrade had not completed the development of his views nor published them in their incomplete form even in articles, their reconstruction is difficult, and I have therefore not attempted such reconstruction in the text.

${ }^{G}$ (p. 225). The International Encyclopedia of Unified Science is published by the University of Chicago Press in the form of separate monographs, the first of which appeared in 1938. Historical information concerning the movement is available in the journal Erkenntnis, continued since 1939 as the Journal of Unified Science.

H (p. 225). Papers dealing with this topic: R. Carnap, "Formalwissenschaft und Realwissenschaft," Erkenntnis, 5, 1935, 30-36; C. Morris, "The Relation of Formal and Empirical Sciences within Scientific Empiricism," Erkenntnis, 5, 1935, 6-14; H. Hahn, Logik, Mathematic und Naturerkennen.

I (p. 227). R. Carnap, "Logical Foundations of the Unity of Science," International Encyclopedia of Unified Science, vol. 1, no. 1, pp. 42-62. On the place of psychology within the system of the sciences, see discussions by R. Carnap, "Psychologie in physikalischer Sprache," Erkenntnis, 3, 1932, 107-42. C. G. Hempel, "Analyse logique de la psychologie," Revue de synthèse, 10, 1935, 27-42; O. Neurath, Einheitswissenschaft und Psychologie. For a behavioral discussion of private experience, see G. H. Mead, Mind, Self, and Society; C. Morris, Foundations of the Theory of Signs, pp. 45-48; 
Six Theories of Mind; B. F. Skinner, "The Operational Analysis of Psychological Terms," Psychological Review, 52, 1945, $270-77$.

${ }^{J}$ (p. 230). The term 'Geisteswissenschaften' includes more than humanistics; it covers what might be called "socio-humanistic studies." Our discussion is applicable to the social studies as well as to humanistics, insofar as these studies concern forms of discourse (such as political discourse) which are not simply scientific. Social sciences, in the sense of scientific studies of social behavior, raise no special problems, since the study of the interactions of organisms is simply one phase of the biological and psychological investigation of animals and men. In this sense there does not seem to be a general problem of the relation of the social sciences to systematized science. Hence we have concentrated upon the incorporation of humanistics within unified science.

${ }^{\mathbf{x}}$ (p. 233). The pragmatic movement, from Peirce through James, Mead, Dewey, and Lewis, has been semiotical in orientation, progressively extending its interest to all modes and usages of signs; Peirce explicitly formulated pragmatism as a logical or semiotical doctrine, and although this emphasis has changed and widened it has never been entirely lost. Such logical empiricists as Schlick, Weismann, and Carnap have developed in various ways Wittgenstein's thesis that "all philosophy is 'Critique of Language'." Thus Carnap writes: "the task of philosophy is semiotical analysis; the problems of philosophy concern-not the ultimate nature of being but-the semiotical structure of the language of science, including the theoretical part of everyday language" (Introduction to Semantics, p. 250). PostKantian idealists, such as Cassirer, Whitehead, and Urban, have in varying manners linked philosophy with the study of the major types of symbolic activity, and so with semiotic. Thus Urban interprets metaphysics as "that activity which is concerned with the interpretation of various symbolic forms, including that of science itself" (Language and Reality, p. 683). In Foundations of the Theory of Signs (p. 59), I spoke of "the identification of philosophy with the theory of signs and the unification of science, that is, with the more general and systematic aspects of pure and descriptive semiotic"an identification which now seems to me questionable for reasons given in the text.

${ }^{\mathrm{L}}$ (p. 234). The Stoics give one formulation of this tradition, a formulation continued by John Locke. The usual tripartite formulation does not sharply distinguish cosmology and metaphysics, a distinction which depends on the difficult differentiation of lexicative and formative discourse. This distinction is, however, implicit in Aristotle and his followers, though not made in semiotical terms, in the distinction between the special sciences and the study of "being." Peirce goes far in making the differentiation semiotically. Thus he says of the term 'being': 
"We do not obtain the conception of Being, in the sense implied in the copula, by observing that all the things which we can think of have something in common, for there is no such thing to be observed. We get it by reflecting upon signs-words or thoughts; we observe that different predicates may be attached to the same subject, and that each makes some conception applicable to the subject; then we imagine that a subject has something true of it merely because a predicate (no matter what) is attached to it-and that we call Being. The conception of being is, therefore, a conception about a sign-a thought, or word; and since it is not applicable to every sign, it is not primarily universal, although it is so in its mediate application to things. Being, therefore, may be defined; it may be defined, for example, as that which is common to the objects included in any class, and to the objects not included in the same class. But it is nothing new to say that metaphysical conceptions are primarily and at bottom thoughts about words, or thoughts about thoughts; it is the doctrine both of Aristotle (whose categories are parts of speech) and of Kant (whose categories are the characters of different kinds of propositions)." (Collected Papers, V, p. 294).

${ }^{M}$ (p. 235). On the relation of philosophy to cultural problems see J. Dewey, Philosophy and Civilization, pp. 3-12. The material on philosophy in the text is in part taken from a paper presented to the Sixth Conference on Science, Philosophy, and Religion (1945) under the title "Philosophy as Symbolic Synthesis of Belief." This conception of philosophy has much in common with Dewey's position, though I should stress somewhat more the relation of a philosophy to the personality of its producer. In the contemporary world, for instance, Dewey, Santayana, and Russell are all philosophers as we have used the term; but while their language reveals all the main usages of signs, Dewey's language is characteristically incitive, Santayana's dominantly valuative, and Russell's basically informative. These differences, occurring within a culture whose insistent problem is the relation of science to values and action, seem to me to be linked with the personalities of the three persons.

${ }^{N}$ (p. 238). Empiricists would do well to formulate empiricism semiotically (that is, as a designative statement about signs, or as an appraisal of certain types of discourse, or a prescription for the use of certain forms of language rather than others). This would help to resolve the frequent ambiguity of the empiricist's contentions. Historically, empiricism, when considered as designative discourse, has at times been formulated as a psychological doctrine (Locke) or as a cosmological doctrine (James): today there is a noticeable tendency to formulate it as scientific theory concerning the origin and limits of signification. This tendency, carefully carried out, has much to recommend it. An empirical philosophy would then be a philosophy whose signs conformed to this theory of signification. Such a 
philosophy would not limit itself to semiotic but would be built upon a scientific semiotic as organon.

${ }^{\circ}$ (p. 240). The work of A. Korzybski and his followers, psycho-biological in orientation, has largely been devoted to the therapy of the individual, aiming to protect the individual against exploitation by others and by himself. Sez Science and Sanity, the proceedings of the first and second Congresses for General Semantics, and the journal, Etc. A Review of General Semantics, edited by S. I. Hayakawa.

P (p. 243). See especially C. L. Stevenson, Ethics and Language, pp. 180191; L. K. Frank, "What Is Social Order?" American Journal of Sociology, 49, 1944, 470-77. Dewey's concern has largely been with the social health of signs rather than with the problems of the individual interpreter of signs. His moral emphasis seems to me a necessary corrective to the merely scientific study of communication, which because of its "objectivity" has always the danger of loading the guns of persons who think of other persons merely as things to be "manipulated." For this danger, and the recognition of the need of a moral orientation if it is to be avoided, see K. Riezler, "What Is Public Opinion?" Social Research, 11, 1944, 397-427.

${ }^{Q}$ (p. 245). For more detailed suggestions as to the way these goals may be implemented see C. Morris, "General Education and the Unity of Science Movement," in John Dewey and the Promise of America, Progressive Education Booklet No. 14, 1939, pp. 26-40; and the book by B. Raup and others, The Discipline of Practical ludgment in a Democratic Society. Oto Neurath has, in his system of Isotype, been especially concerned with the importance of the visual iconic sign for education and communication; see references given in the Bibliography. In my opinion the proper use of semiotic in general education would provide the basis for a democratically integrated education at once scientific and humanitarian, and give to the forces of progressive education the instrument and discipline which they have hitherto lacked. 


\section{SOME CONTEMPORARY ANALYSES OF SIGN-PROCESSES}

\section{The History of Semiotic}

QINCE sign-processes play such a large part in human life, it is not $\checkmark$ surprising that man should turn his attention to them at a fairly early date. Semiotic has a long and interesting history. Portions of it are to be found in the histories of linguistics, rhetoric, and logic, though the history of semiotic as a whole yet remains to be written. Even though we neglect here the developments in India, China, and Islam, the depth and persistence of this tradition may at least be indicated by reference to Western civilization. ${ }^{1}$

The term 'semiotic' goes back to the Greek medical tradition which considered semiotic, embracing diagnosis and prognosis by signs, as one of the three divisions of medicine. The Stoics gave semiotic the dignity of a basic division of philosophy co-ordinate with physics and ethics, and included within it logic and the theory of knowledge. The whole of Hellenistic philosophy centered around semiotic, and in particular the problem of empiricism versus metaphysics was formulated as a problem of the limits of signifying by signs, the Stoics arguing that there were signs ("indicative signs") which could give necessary : knowledge about things beyond the limits of observation; the Epicureans holding that while signs gained their signification through experience, some signs (such as 'atom' and 'void') could, though only with probability, refer to what was not capable of direct observation; the Sceptics questioned the whole edifice of metaphysics on the ground that signs

\footnotetext{
${ }^{1}$ For some phases of this tradition see Philodemus, by P. H. and E. A. DeLacy (Philological Monographs, 1941); Geschicte der Logik im Abendlande, by Carl von Prantl; Les Oeutres de Siger de Courtrai, by G. Wallerand (Louvain, 1913); La Logique de Leibniz d'après des documents inédits, by Louis Couturat (Paris, 1901).
} 
could refer only to that which was observable, serving to recall (as "commemorative signs") that which had been observed even though it was not at the moment of reference directly observable.

The Hellenistic development of semiotic had been prepared by the analyses of Aristotle, which in turn made use of the material furnished by Plato, the Sophists, and the medics. Aristotle was attentive to many phases of semiosis: in the Organon, to the semantics and syntactics of the language of science; in the Poetics, to the various aspects of esthetic discourse; in the Rhetoric, to some problems of pragmatics. The fact that he approached semiotic in terms of his own theory of mind, a theory confusedly scientific and metaphysical, influenced for centuries the psychological and philosophical context in which semiotic was embedded.

Through such figures as Augustine and Boethius, the Aristotelian and Hellenistic theories of signs (including the works of the classical linguists) passed over into medieval Europe. There was developed by Petrus Hispanus, Abelard, Roger Bacon, Thomas of Erfurt, Siger of Courtrai, William of Ockham and others a comprehensive and subtle theory of signs (known as scientia sermocinalis) which embraced grammar, logic, and rhetoric. Within this development two streams can be distinguished: the dominant tendency was to interpret signprocesses within the framework of the Platonic and Aristotelian metaphysics; this was countered by a progressively growing attempt to assimilate semiotic to empirical science and philosophy. The first direction was carried on by Leibniz, the second by the British empiricists.

Leibniz is one of the major figures in the history of semiotic, and of syntactics in particular. Though he upheld a rational psychology, he believed that the signs the mind used presented in a perceptible and more easily studied form the thought-structure and the world-structure which they mirrored; hence his main attention in semiotic was given to the syntactical study of sign-structures. In his work the Scholastic study of language was given a new direction through the impact of mathematics conceived as a formal system of signs, a view which in its development owed much to Vieta. It is interesting to note that Leibniz thought of even the calculus as only a part of his wider theory of signs and an illustration of its fruitfulness. Leibniz' ideas of a universal 
system of signs (the characteristica universalis) and of a formal calculus applicable to all reasoning whatsoever (the calculus ratiocinator) have been developed by the symbolic logicians and the unity of science movement. Such persons as Boole, Frege, Peano, Peirce, Russell, Whitehead, Sheffer, Carnap, and Tarski have carried forward the syntactical emphasis which was central in Leibniz.

: The British empiricists were concerned primarily with semantics, as envisaged within their own characteristic version of empiricism. Francis Bacon, Hobbes, Locke, Berkeley, Hume, and Bentham developed analyses of signification in terms of a psychology which progressively became individualistic and sensationalistic. While their specific analyses of the experiential origin and reference of many terms was acute, the psychology they held entangled semiotic within the epistemological problems which the mentalistic associational psychology provoked. It should be mentioned that Locke adopted the Stoic term 'semiotic.' The approach to semiotic of Ogden and Richards continues the English development under the direct influence of Bentham.

The American pragmatists have been concerned mainly with a study of signs within human behavior. This behavioral orientation of semiotic, the central motive in the pragmatic movement, was initiated by Charles Peirce.

\section{Charles Peirce on Signs}

Peirce was the heir of the whole historical philosophical analysis of signs and has himself had a major influence upon contemporary discussion. He explicitly connected sign-processes with processes involving mediation (or "thirdness") and indeed often equated such processes. Peirce writes: "It is important to understand what I mean by semiosis. All dynamical action, or action of brute force, physical or psychical, either takes place between two subjects . . . or at any rate is a resultant of such actions between pairs. But by 'semiosis' I mean, on the contrary, an action, or influence, which is, or involves, a coöperation of three subjects, such as a sign, its object, and its interpretant, this trirelative influence not being in any way resolvable into actions between pairs. . . My definition confers on anything that so acts the title of a 
'sign." 2 The act of giving something to someone would for Peirce be an example of a genuine triadic relation involving mediation, and irreducible to a combination of dyadic relations between pairs of objects-thus no "giving" is involved if an object in an extended hand of one person is simply taken by another person. Peirce always connects processes of mediation, sign-processes, and mental processes. This means that he would not accept any behavioral psychology which attempted to reduce behavior to two-term relations between stimuli and responses. And recent formulations of behavior theory have in their own way accepted Peirce's position in so far as they recognize that the conditioning of one response to a stimulus which previously produced another response is mediated by a third factor, a "reinforcing" state of affairs in which a need of the animal is reduced or satisfied. Thus Hilgard and Marquis" define a conditioned response as "a response which appears or is modified as a consequence of the occurrence of a conditioned stimulus in proximity to reinforcement." In Peirce's sense, such conditioning is then triadic, and the reinforcing state of affairs is the factor of mediation.

Yet Peirce's definition of sign is much wider than the one here proposed. Peirce regards any genuine process of mediation as involving signs, and so mental processes of some degree or other. But it would seem that this is a doubtful or unwise use of terms. For if all conditioning is mediated by a relation to a reinforcing state of affairs, then every conditioned stimulus would become a sign-a position at variance with ordinary usage. And, further, there seem to be many processes involving mediation other than conditioning which would not ordinarily be regarded as signs: the eye, for example, in seeing is a genuine mediating element in the relation of certain responses to certain objects, and yet the eye (or even the retinal image) would hardly be called a sign in such cases. Hence it seems advisable to delimit in some way sign-processes within the general class of processes involving mediation, and we have done this by restricting sign-processes to those in which the factor of mediation is an interpretant.

On Peirce's formulation sign-processes (and so mental processes) are,

- Collected Papers, V, $\$ 484$.

Conditioning and Learning, p. 342. 
however, not even restricted to behavior situations. "Thought," he writes, "is not necessarily connected with a brain. It appears in the work of bees, of crystals, and throughout the purely physical world." 4 Hence his very general definition of sign-one which philosophically is used by Peirce (and by Royce) to support an idealistic metaphysics: he defines 'represent' as "to stand for, that is, to be in such a relation to another that for certain purposes it is treated by some mind as if it were that other." Or again: "As representation is that character of a thing by virtue of which, for the production of a certain mental effect, it may stand in place of another thing. The thing having this character I term a representamen, the mental effect, or thought, its interpretant, the thing for which it stands, its object";" "A sign is a representamen of which some interpretant is a cognition of a mind. Signs are the only representamens that have been much studied." 7

This material is quoted to illustrate the difficulties which appear when we leave the ground of behavior situations in attempting to define 'sign.' For if 'sign' is defined in terms of mind or thought, then we cannot furnish an empirical criterion for determining whether a certain thing is or is not a sign until we have a satisfactory criterion for the occurrence of mind or thought. It seems that Peirce does not give such a criterion in a way usable by science. It should be noted that Peirce's own various formulations differ considerably. At times he writes as if "every thought is a sign"; ${ }^{8}$ at times merely that thinking never occurs without the presence of something which serves as a sign. ${ }^{9}$ And if 'interpretant' is at times defined in terms of "mental effect or thought," the fullest accounts of it are couched in terms of habit. ${ }^{10}$ The present treatment follows Peirce's emphasis upon behavior rather than his more mentalistic formulations. Hence it not only avoids the extension of signprocesses to inorganic nature, but does not require that all behavior involves sign phenomena.

- Collected Papers, IV, $\$ 551$.

${ }^{5}$ Ibid., II, \$273.

- Ibid., I, \$564.

"Ibid., II, \$242.

${ }^{8}$ Ibid., v, $\$ 253$.

'Ibid., v, \$283.

${ }^{10}$ Ibid., IV, \$536; V, $\$ 475 \mathrm{ft}$. 
There is another point to be mentioned. Peirce almost always defines 'sign' in such a way that the interpretant of a sign is itself a sign, and so ad infinitum. One such formulation is the following: "A Representamen is a subject of a triadic relation to a second, called its object, for a third, called its Interpretant, this triadic relation being such that the Representamen determines its interpretant to stand in the same triadic relation to the same object for some interpretant." 11 Or more succinctly: "It is essential to a representamen that it should contribute to the determination of another representamen distinct from itself." 12 This seems to confuse the problem of the definition of 'sign' with the empirical question as to whether signs always generate new signs. Unless these are distinguished a circularity is introduced into the definition, for a sign is defined as something which generates a sign which generates a sign, etc., and this is certainly objectionable as a form of definition of 'sign' itself. Signs, at least at the human level, do frequently generate a series of sign-processes, but I see no reason why this fact about signs should be incorporated into the definition of 'sign' itself.

These remarks are not to be taken as an adequate presentation or assessment of Peirce's work in semiotic. His classification of signs, his refusal to separate completely animal and human sign-processes, his often penetrating remarks on linguistic categories, his application of semiotic to the problems of logic and philosophy, and the general acumen of his observations and distinctions, make his work in semiotic a source of stimulation that has few equals in the history of this field. The very existence of this material shows that significant analytical work in semiotic does not depend upon a specifically behavorial theory of signs, and indeed may set significant problems for such a theory. On the other hand, the difficulties which have been mentioned indicate the importance of such a grounding of semiotic if it is to become scientific. Peirce's account of signs is embedded in the metaphysics of his categories (possibility, existence, and law are the basic terms in his classification of signs) and in the metaphysics of his view of mind. These are not secure bases for a scientific semiotic. But Pierce himself, in his

${ }^{11}$ Collected Papers, I, $\$ 541$.

${ }^{12}$ Ibid., V, $\$ 138$. 
rejection of the older Cartesian mentalism and in the place he assigns to habit in his account of signs, has at least indicated a possible direction of advance toward a more adequate account of sign phenomena.

\section{Behavioral Formulations of Sign-Processes}

It was characteristic of the early behaviorists to neglect the goaldirected character of behavior, that is, to forget that the stimulusresponse connections in which they were interested occur (or usually occur) as factors within a set of responses motivated by a need and directed to the securing of goal-objects which would satisfy the need. And with this neglect of behavior-families no adequate treatment of the sign was possible. A sign-if the term was used at all-would be either any stimulus or (as in Pavlov's use of 'signal') any conditioned stimulus, and neither of these translations is narrow enough to fit the common usages of the term 'sign.' The problem for later behavior theory thus became that of finding an objective criterion for signs which would distinguish signs from stimuli in general, and from conditioned stimuli in particular.

H. L. Hollingworth sought this differentiation in the concept of redintegration. In redintegration a part of a sequence of events is said to produce the consequence which previously was produced by the whole sequence of events of which it was a part. Such a part he calls a sign or symbol. ${ }^{13}$ In his words, "A complex antecedent, $x y z$ instigates a consequent $c$. . . . Thereafter, if it occurs that consequent $c$, or one belonging in the same class, is instigated by detail $x$, or a detail belonging in the same class, by virtue of the historic participation of $x$ in the situation $x y z$, we have a case of meaning. The fact of meaning is the determination of $c$ by the previous context of $x$. ." 14 The behavior factor is then introduced as follows: "Encountering any of these 'symbols,' if I behave, or if anything else behaves, in terms of the wholes of which they are or once were parts, we have an example of a mental phenomenon. The events are not mere occurrences. They have 'acquired meaning,' they are now

\footnotetext{
13 "Meaning and the Psycho-Physical Continuum," Journal of Philosophy, 20, 1923, p. 436; this article is reprinted in his Psychology of Thought.

"lbid., p. 439.
} 
'thoughts' as well as events. Meaning is the determination of subsequent events in the light of the history of the antecedent detail. Thoughts are events of any sort whatsoever that function for larger antecedent contexts." 15

What is interesting in this account, and in the whole psychology which Hollingworth erected upon it, ${ }^{16}$ is the fact that 'thought' and 'mind' are defined in terms of 'sign' rather than being used in the definition of 'sign'; what is lacking, however, is precisely the clear distinction between sign and conditioned stimulus which is wanted, for a conditioned stimulus is always one element in a total complex, and so seems to meet the requirement for redintegrative (or "part-whole") efficacy. H. Cason has claimed that "a redintegration is the same as a group of conditioned responses." 17 And present behavior theory tends to limit redintegrative response to one special case of conditioned response, namely that where the conditioned response is very similar to the unconditioned response. ${ }^{18}$ Thus if the buzzer called out responses very similar to what the food called out, such responses would be called redintegrative. But such highly similar responses in fact very seldom occur, and their occurrence is certainly not essential for something to be called a sign. It would seem that Hollingworth has not sufficiently explicated the way in which signs "function for larger antecedent contexts."

Walter S. Hunter has performed a number of experiments on "delayed reactions" which aim to isolate objectively sign-behavior from other reactions, and has in a number of papers tried to differentiate signs from conditioned stimuli and to then define 'consciousness' in terms of sign-process. Three of these papers appeared in the 1924 and 1925 volumes of the Psychological Review; the most important for our purposes is "The Symbolic Process" (1924). A symbolic process is there defined ${ }^{19}$ as "a substitute process which can be reinstated by the organism, but only where associative traces of the original process per-

\footnotetext{
${ }^{15}$ Ibid., p. 436.

${ }^{18}$ In his Psychology, Abnormal Psychology, and other volumes.

17 "General Aspects of the Conditioned Response," Psychological Review, 32, 1925, p. 316.

${ }^{28}$ E. R. Hilgard and D. C. Marquis, Conditioning and Learning, p. 37.

10 "The Symbolic Process," Psychological Review, Vol. 31, 1924, p. 488.
} 
sist in the integration"-when such traces are lost the symbol becomes a bare conditioned response. Thought or consciousness is then defined in terms of the presence of symbolic processes.

Hunter's account of sign-processes is certainly compatible with the position which has here been taken with respect to symbols though it is not sufficiently elaborated for one to be sure whether the two definitions are identical. Hunter succeeded in isolating an essential feature of symbol situations: namely, that a response to the situation must be dependent upon the making of another response. His account seems then in its direction, if not in its exact formulation, very close to the present definition of symbol-processes. And it makes intelligible the contention that while conditioned responses may be involved in signprocesses, a sign is not simply a conditioned stimulus and an interpretant is not simply a conditioned response. The type of behavior which Hunter called a delayed response often does involve a symbolprocess, though there is considerable doubt as to whether this is always the case. His experiments on such responses are still of importance in determining the existence of symbols in sub-human animals, and have set the pattern for much later experimentation. Hunter's designation of all sign-processes as "language responses," however, is not acceptable, since it blurs certain important differences between language signs at the human level and signs which occur in the behavior of other animals.

Recent behavior theory has been forced to consider the goal-directed character of behavior in order to explain many cases of conditioning. Suggestions that it is necessary to introduce considerations of goals into the definition of 'sign' have appeared in a number of writers. Bertrand Russell, who previously had tried to explain meaning in terms of images, has in later years introduced the notion of "appropriate behavior" into his account. In An Inquiry into Meaning and Truth are these words: "We may say that $A$ is a 'sign' of $B$ if it promotes behavior that $B$ would promote, but that has no appropriateness to $A$ alone." 20 He himself finds a difficulty in this formulation, "because there is no satisfactory definition of appropriate behavior." $H$. Gomperz puts the

"o Page 13. 
matter in slightly different terms: "For a person $P$ an object or fact $s$ is said to function as a sign for another object or fact $o$ in so far as $P^{\prime} s$ response to $s$ is similar to what would have been $P$ 's response to $a$, provided $a$ ) that this similarity of response is not fully accounted for by the similarity of $s$ and $o$ and provided further $b$ ) that $P^{\prime} s$ response to $o$ would in this case have been understandable as natural or reasonable by itself, without any reference to $s . "{ }^{21}$

In both of these formulations the essentially new element is the insistence that the response called out by a sign must be appropriate to something else than the sign itself. This may be taken in two ways, one of which underlines a point which a formulation of sign in terms of behavior must not neglect, while the second seems to involve a confusion which has been noted. There is no special difficulty in defining 'appropriate response'; a response to $x$ may be said to be appropriate for the realization of goal $y$ to the degree that the response is such that $x$ furthers the realization of goal $y$. Thus breathing carbon monoxide gas in an inclosed room for a certain length of time is appropriate behavior to carbon monoxide for a person who wishes to commit suicide. Now if such a person made a test for the presence of the gas, the test condition would be a sign of the presence (or absence) of the gas, and appropriate behavior to the sign would not consist in responding to the sign as if it itself were the gas; in general the response to a sign is, as we have seen, merely a factor in causing a response to something else. But to require that the response involved in a sign-process be appropriate to something other than the sign is, as we have earlier seen, to confuse the existence of signs with questions as to their adequacy (that is, to confuse semantics and pragmatics). It is frequently true that events do not continue to function as signs unless they advance behavior in the situations in which they appear (that is, unless the response-sequences which they help to initiate can be carried to completion), but this is a truth about signs and not an element in their definition. Hence the conclusion that the criterion of appropriateness of response is not itself sufficient to isolate those responses involved in sign-processes from those which are not involved.

21 "The Meanings of 'Meaning'," Philosophy of Science, 8, 1941, pp. 160-61. 


\section{Mentalistic Formulations of Sign-Processes}

The preceding pages have made evident the fact that it is not easy to translate the ordinary usages of 'sign' into terms describing a behavior situation. Hence it is understandable that many persons should have doubt as to this procedure, and should adopt definitions couched in such terms as 'mind,' 'thought,' 'belief,' 'consciousness.' Several of these attempts may be mentioned so that the issues involved may become clearer.

C. K. Ogden and I. A. Richards developed in The Meaning of Meaning what they called the context theory of meaning. Richards has formulated this doctrine as follows: "A sign . . . works by being a member of a certain kind of interpretative context in the mind-let us write it $a b c q$. When $a b c$.. . recurs without $q$-such is the fortunate and overwhelming important characteristic of these interpretative contexts-the effect is in certain respects as though $q$ also had recurred. And $q$, then, is what $a$ (the sign) is of, what it signifies, or represents." 22 This contextual position has much in common with Hollingworth's concept of redintegration, except that where Hollingworth defines mental process in terms of sign-process, the OgdenRichards account brings in mental process into the definition of sign itself. This was true even in the first edition of The Meaning of Meaning, though there the formulation was more compatible with a behavioral translation: thus it was held that "we should develop our theory of signs from observations on other people, and only admit evidence drawn from introspection when we know how to appraise it," ${ }^{23}$ and the "peculiarity of interpretation" was stated to lie in the fact that "the recurrence of merely a part of the context will cause us to react in the way in which we re-acted before." ${ }^{24}$

Richards' later statement that "meaning is delegated efficacy" 25 moves in the same direction: one would expect an account of how the sign exercises this delegated efficacy, and our formulation attempts this

\footnotetext{
20 "Preface to a Dictionary," Psyche, 13, 1933, p. 18.

"The Meaning of Meaning, p. 26.

${ }^{24}$ Ibid., p. 139.

${ }^{25}$ The Philosophy of Rhetoric, p. 32.
} 
explanation. But Richards himself has not followed this behavioral lead; he has more and more invoked "thought" in his account of sign, and shown greater scepticism as to explaining thought in behavioral terms. Thought, he says, is not muscular movement, and while it may be an activity of the nervous system we know too little about the nervous system to make such an identification: "We can all detect a difference in our own minds between thinking of a dog and thinking of a cat. But no neurologist can. Even when no cats or dogs are about and we are doing nothing about them except thinking of them, the difference is plainly perceptible. We can also say 'dog' and think 'cat'." ${ }^{26}$ Richards concludes that "no one knows" how signs work. ${ }^{27}$

This shift in emphasis from the objectivistic strain in The Meaning of Meaning is significant in showing how problems connected with thought and introspection often intrude themselves into attempts to formulate the nature of sign-processes. But in this case at least-and I believe in all such cases-the scientific problem is not advanced unless introspective data are merely taken as one (and logically subordinate) type of evidence for affirming the existence of sign-processes. This is not to impugn the great significance of the later work of Ogden and Richards, which falls mainly in the sphere of the educator's interest in semiotic; but it does suggest that this work has not advanced the fundamental scientific problems which are our present concern. The value of the stress upon context is better preserved by setting sign-processes within the context of behavior situations. In this context-and not by reference to "mind" or "thought"-is to be explained the "respects" in which signs serve as surrogates for situations and the way in which they acquire "delegated efficacy."

The introduction of the categories of mind and belief into the account of sign-processes may also be illustrated by G. Watts Cunningham's paper, "Perspective and Context in the Meaning-Situation." ${ }^{28}$ Cunningham distinguishes five factors in the meaning-situation: a reference, a content which refers, the referent which is referred to, the perspective for which the reference holds, and the context because of which the

\footnotetext{
${ }^{26}$ The Philosophy of Rhetoric, pp. 13-14.

${ }^{27}$ Ibid., p. 34.

${ }^{2}$ University of California Publications in Philosophy, 16, 1935, pp. 29-52.
} 
reference holds. "A meaning-situation," he writes, "is one in which a content refers to a referent for a perspective and because of a context." ${ }^{29}$ It is the perspective only which might seem to introduce a new factor into our account. The perspective is said to be a mind, and to involve a body of beliefs personally held or operative- - "for it seems fair to say that only in respect of some body of beliefs may anything refer meaningfully to anything else or be said to have meaning." 30 Meaning or meaning-situations, while not completely mental or in a mind, are thus felt to involve thought and belief as necessary elements.

This same insistence upon mental categories in the definition of sign is found in Aristotle and the Scholastics, in many of the British empiricists, and in thinkers as diverse as Brentano, Husserl, Cassirer, and Urban. It is specifically introduced by C. J. Ducasse as a challenge to the type of analysis which the present volume represents.

Ducasse's own analysis of signs is given in an article "Symbols, Signs, and Signals." 31 There he argues that "interpretation is the kind of mental event consisting in this, that consciousness of something causes us to become conscious of something else," and such interpretation is regarded as "semeiotic interpretation" when it involves "regularity of causation." ${ }^{32}$ We will not, however, discuss here his own phenomenological analysis, which seeks to make no reference to "the interpreter's public behavior," but single out the reasons why he believes that an account in terms of "public behavior" is inadequate. This is given in his penetrating article, "Some Comments on C. W. Morris's 'Foundations of the Theory of Signs'." ${ }^{33}$

In this article he writes as follows: "If we insist on defining 'sign of' as a function of a person's perceptually observable behavior, the definition has to be this: $S$ is a sign of $D$ for (an interpreter) $I$ to the degree that the presence of $S$, conjointly with purposes of kind $P$ in 1 and with belief by $I$ that the circumstances are of kind $C$ and with belief that behavior of kind $B$ in such circumstances would promote these purposes, causes $I$ to behave in manner $B$. Nothing less than this will

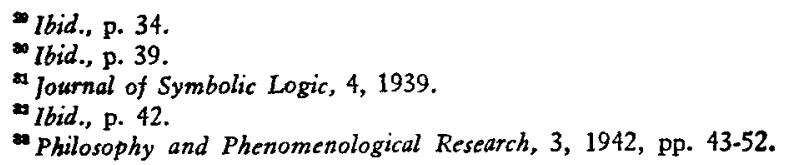


do. But although in this definition the person's public behavior is one of the variables of which the status of $S$ as for him [a] 'sign of' $D$ is a function, this status is a function also of three other variables, all of them mental and therefore private; for belief of something by a person cannot be defined in terms solely of his public behavior, but the definition has to include reference to his purposes; and a person's purposes are directly knowable only by him, through introspection. Other persons can know them, if at all, only indirectly and later, through more or less precarious inferences from his verbal behavior, or from his operatory behavior if he attempts to realize them by means of bodily movements. I conclude that a definition of semiosis in terms solely of public behavior, such as Morris attempts, not only is as he suggests not necessary, but is impossible; and that the definition in such terms, which he proposes, owes what plausibility it may have only to the fact that most readers tacitly make the required supplementary assumptions as to private states-the purposes and beliefs- ${ }^{-}$of the interpreter." ${ }^{34}$

Ducasse's view, then, is that semiosis has to be defined with reference to belief and purpose, and that it may, but need not, involve a further reference to the interpreter's "public behavior." Beliefs and purposes are themselves held to be private mental states. Only if the term behavior includes "any response, whether bodily or mental" is a behavioral analysis of semiosis held to be adequate.

If Ducasse's formulation were applied to the situation which has been taken as a paradigm, he would presumably say that the buzzer is a sign to the dog of food only if the dog has the purpose of securing food, and believes that food exists. Now in so far as this is merely an assertion that sign-processes occur within situations in which animals respond in ways which normally meet their needs, there would be no essential difference between Ducasse's suggested emendation of my previous account and the account given in the present study. A difference would arise only when it is further claimed that beliefs are mental and private in a sense which prohibits an objective observational determination of whether an animal does or does not have a certain

24 lbid., pp. 46-47. 
belief. Then the questions arise whether beliefs are antecedent to the appearance of signs (as Ducasse seems to suggest), or whether the appearance of a belief and preparedness to respond in a certain way are the same thing, or whether beliefs are new elements consequent upon the appearance of signs. Unless 'belief' is analyzed in a way which permits of sufficient agreement for the determination of such questions, its introduction into the definition of sign obscures rather than facilitates the definition. Behavioral formulations of 'belief' are certainly possible, though Ducasse seems to dismiss this possibility. But whether formulated objectively or in some other terms, it still does not follow that even if all sign-processes occur only in a context of belief, and require beliefs as their preconditions, that a sign is to be defined with reference to such beliefs. Two animals might differ in their "beliefs" as to how a certain object might relate to their purposes and yet the same object be signified for each animal. It is important to stress the embeddedness of signs in behavior situations; it is doubtful whether it is necessary or desirable to introduce the term 'belief' in describing such situations; it is certainly questionable, if the term is introduced, whether it must be defined as designating a private mental state.

\section{Consideration of the Behavioral-Mentalistic Controversy}

The preceding differences between definitions of signs in terms of behavior situations and definitions in terms of mentalistic categories is not so unsettling for semiotic as it may at first appear. Genuine issues are involved no doubt, issues that concern, however, more the state of psychology as a science than semiotic itself.

In our everyday language the terms 'experience,' 'sensation,' 'awareness,' 'consciousness,' 'thought,' 'mind,' 'belief,' 'purpose,' 'introspection' frequently occur. There is no reason for the semiotician to suppose that these terms are any less signs than other terms such as 'organism' or 'rock.' The questions as to what they signify and the degree to which their significations are interpersonal are simply one set of problems among others. There are psychologists who take these terms, or some of them, as primitive and undefined terms, while some psychologists 
attempt to formulate them in terms descriptive of behavior situations. Thus Mead has given an analysis of many of these terms, and Tolman and Hull have done the same. To say as Ducasse says that belief and purpose are "private mental states" would be to a follower of Tolman, for instance, a highly arbitrary assertion. Similarly, few behavioristicians would deny that a person can observe himself, or that some experiences are accessible to self-observation in a way not accessible to other observers-after-images, pain, and dreams would be cases in point. And certainly the semiotician is not called upon to reject observation of his own sign-processes or the reports of others of their observation of their sign-processes. The theoretical question is how what is observed in self-observation is to be described. To enter into this conflict is to assume the burden of the psychologist.

The semiotician is interested in these complex methodological and scientific problems only in so far as they concern his task. The question for him is simply how he had best introduce his basic terms in order that a science of signs may be obtained, that is, a systematized body of statements from which deductions may be made that can be controlled by observation. Unless his terms are linked with observable phenomena he has no natural science at all. The choice of the terms in which to define the terms of a scientific semiotic is determined by the scientific goal of getting the maximum objective knowledge possible; those interpersonal terms will be chosen upon which there is the greatest agreement in usage. The mentalist terms do not meet this test. This is evidenced by the fact that many psychologists have come more and more to regard them as designating complex phenomena, and to introduce them late in their terminological hierarchy and not as primitive terms. The fact that the theory of signs has for thousands of years been couched in such terms without attaining a scientific status should raise strong doubts about their continued use as primitive terms for semiotic. On the other hand, the study of behavior has been developing cumulatively in recent decades and is becoming an advanced experimental science. Hence the naturalness of seeking in it the definition of the basic terms of semiotic. Whether behavior theory is or is not psychology is not in this respect an essential problem for the semiotician. 
It is for these reasons, and these alone, that we have sought to link semiotic with the science of behavior. Hence we have interpreted its basic primitive terms in words used to describe behavior situations. Others can if they wish not define these terms at all, or define them in mentalistic terms; in the first case no natural science results, in the second case one will have to see what significant results are obtained and history does not suggest much ground for optimism.

There is one further point of interest, though not directly relevant to the development of semiotic itself. There is a tendency among certain psychologists, such as Hollingworth, Hunter, Mead, and others, to define some of the traditional psychological terms-such as 'mind,' 'thought,' and 'consciousness' - on the basis of a sign terminology, rather than the other way around. This suggests the possibility that some portion of psychology, and perhaps even the very delimitation of the field of psychology (as, for instance, the science of sign-behavior) may depend upon an adequate doctrine of signs and be helped by its development. This warrants caution in choosing highly complicated psychological terms as the foundations for semiotic.

The same point might be made with respect to philosophy. For centuries semiotic has been developed within the framework of various systems of philosophy, and even today many philosophers seem to feel that the semiotician must first solve the standard problems of the theory of knowledge and metaphysics before considering sign-processes themselves. Such persons will feel that we should first have defined terms like 'experience' and 'object' before talking about organisms, food, and signs. Now these terms may, of course, be analyzed, and philosophy should give their analyses, but history again shows that they are very complex terms and their analysis very difficult. Hence it is hard to see why semiotic should set itself this analysis as an initial problem, any more than should physics or anthropology. It may well be that the philosophical task itself will be facilitated by a more fully developed semiotic. And there is no reason why such a development must be delayed until philosophy and psychology have put their own houses in order. Modest jobs done well on simple phenomena first-such is the way a science proceeds. 


\section{Tolman's Concept of Sign-Gestalt}

Among all behavior theorists, Edward C. Tolman has given the category of sign the most prominent place. All behavior-though not all responses-becomes sign-behavior, and the concept of sign replaces in this field the concept of conditioning. Perception, inference, memory, feeling, emotion, and personality mechanisms (such as repression and fixation) are all interpreted as sign phenomena. In this way a whole psychology is erected, as in the case of Hollingworth, on the basis of the concept of sign, and sign-processes are specifically described in terms of behavior situations.

According to Tolman a sign-gestalt is said to be present when three conditions are fulfilled: (1) there must be features of the behavior situation to which the animal responds (these he calls signs or sign-objects), (2) there must be objects signified by these signs (these he calls significates), (3) there must be signified the relation between the signs and their significates, that is, "the manner in which, on the previous occasions, the commerces with the signs led on to the commerce with the significates" (these he calls signified means-end-relations or, later, signified utilitanda). ${ }^{35} \quad$ A sign is in this account always a means-object, the significate is always a goal-object, and a sign-process always involves a signified relation between commerce with the means-object and access to a goal-object. All behavior is regarded as involving the operation of innate sign-gestalts or the correction and formation of sign-gestalts: "Taking all the evidence into account, it appears to the writer that learning must be conceived, not as the establishment of simple $S-R$ connections, but rather the establishment of $S-r-s$ wholes, where $r$ and $s$ may be defined as "expectations" that, if $S$ is responded to in the manner $r$, the effect $s$, will result. And then the actual performance, $R$, on a given occasion will depend upon whether or not this expected after-effect, $s$, will be satisfying or not (given the controlling wants, interests, attitudes at the moment)." 36

It is evident from the above quotation, as well as from Tolman's frequent use of the phrase 'sign-gestalt expectation,' that the "signifying"

${ }^{*}$ Purposive Behavior in Animals and Men, p. 136.

36 "Connectionism; Wants, Interests, and Attitudes," Character and Personality, 4, 1936, p. 249. 
which appears in his definition of 'sign' is to be behaviorally interpreted in terms of 'expectation.' At times the term 'readiness' is used in such phrases as "sign-gestalt readiness," but 'expectation' seems to remain the basic behavioral category in his account of signs. This is evidenced in the following statement: "Sign-gestalt-expectations we have defined as readinesses of the organism to expect certain types of resulting significates as lying in such and such types of means-end-relation with respect to such and such given, immediately presented, signs." ${ }^{37}$

The central problem then becomes that of specifying the conditions under which an animal may be said to have an expectation. Critics of Tolman's position often state, and with some justice, that expectancy is too anthropomorphic a concept, and that its use leads the reader to attribute to animals what in fact only occurs at the level of human beings equipped with language. That there is this danger cannot be doubted; but also it cannot be doubted that Tolman does not intend such an interpretation of his doctrine. He explicitly states that a sign-gestalt expectation does not imply a conscious process but is a "set" in the animal "defined by the fact that it leads to a certain type of behavior in the given situation and that it only continues to lead to this behavior so long as a certain arrangement of objects in the environment does actually obtain." 38

In this respect Tolman's doctrine of signs does not differ in essentials from the doctrine here defended, for the ultimate test of an expectation, regarded as a "set" in an animal, is whether or not a certain kind of behavior occurs in a given situation. On the present account this behavior must involve a preparatory set; can 'expectation' then be translated by 'interpretant'? In favor of this translation is the fact that if 'expectation' be used in a wider sense, such as for the making of a response to something in advance of the appearance of that something (such responses being often called anticipatory responses), then expectations often occur in cases which would not normally be called signprocesses (as where one blinks before an approaching object touches the eye) and do not occur in cases which would normally be called sign-

\footnotetext{
- Purposive Behavior in Animals and Men, p. 258.

* "The Acquisition of String-Pulling by Rats-Conditioncd Response or Sign-Gestalt?" Psychological Review, 44, 1937, 207 n.
} 
processes (as when one reads a letter about a war in China and makes at the moment no detectable response of the sort one would make if at the described battle field). This suggests that expectations, in the only sense relevant to sign-processes, are interpretants, i.e., readiness to act in a certain way to the environment because of something else in the environment, such readiness to be ultimately tested by whether the response in question occurs under supporting environmental conditions. This formulation has the merit of keeping Tolman's insight that signs do not necessarily involve anticipatory responses, and yet of bringing his account into basic agreement with the position taken in this volume. Whether or not such translation is fair to his account must be left to him; if it is not, Tolman's responsibility is to clarify further the category of expectation and its relation to signs.

It will be recalled that Tolman includes two factors in his formulation of signifying: the sign is said to signify an "object" (the significate) and to signify means-ends-relations (utilitanda properties). Thus the buzzer sound would be said to signify food, but food as something to be obtained by responding in certain ways to objects in the environment as means-objects. This recognition that objects enter into behavior situations as things to be responded to in ways relevant to the goals of behavior is of basic importance. There is, for instance, no evidence that the buzzer sound signifies food in the highly general sense that is signified by the word 'food.' Behaviorally we can merely say that the animal is responding, because of the buzzer, to certain properties of the situation to which it would not otherwise respond. Hence while it is necessary to distinguish the different aspects of behavior situations which are signified-as is done in the distinction of modes of signifying-it is somewhat confusing to exclude from the term 'significate' the signifying of utilitanda properties. Hence the present account includes such properties, when signified, under the term 'significatum.' This avoids the confusing implications of Tolman's statement that in a sign-process there is "signified the relation between the signs and their significates," for this suggests that in every sign-process the sign is itself signified. But this would attribute to non-human animals a complexity of sign-processes probably found only-and then only occasionally-at the human level. Here again it is necessary to avoid attributing to an 
animal the distinctions made at the level in which sign-processes are analyzed and described.

The above point is connected with Tolman's view that a sign is always a means-object. There is a sense, of course, in which the buzzer is a means-object, and "commerce" with it leads on to its significate, and yet this is a very special sense. For the genuine commerce is with the food which is signified, rather than with the buzzer; this is even more evident at the human level in which the word 'buzzer' has appeared. Even in those cases where the object which is a sign is itself something which must be responded to in a certain way if the goal is to be attained, it is some object itself, and not the object in its capacity as a sign, which is the means-object. In the strict sense, a sign is not always a means-object, and conversely, not all means-objects are signs.

Tolman's merit is to have gone very far in the direction of isolating the special kind of behavior here called sign-behavior. As his formulation stands it is open to the confusions which have been discussed. Tolman has nevertheless done much to make plausible the central place which sign-behavior plays in psychology, and to advance semiotic as an experimental behavioral science.

\section{Hull's Concept of Pure-Stimulus Acts}

Clark L. Hull and his associates have been especially concerned with a detailed study of the mechanisms operative in behavior. With respect to signs, Hull, in his earlier papers, suggested that the physical basis of sign-processes was to be sought in what he called a pure-stimulus act. While his later writings have tended more and more to avoid such terms as 'sign' and 'symbol'-or, as he has stated in conversation, to restrict these terms to the genuinely linguistic level-there is much of significance in the earlier suggestions; the pure-stimulus act, while not itself necessarily symbolic, does seem to be an essential element in symbol-processes (though not in signal-processes).

Pure-stimulus acts were defined as "acts whose sole function is to serve as stimuli for other acts." ${ }^{39}$ An example, at a complex level, are

\footnotetext{
" "Knowledge and Purpose as Habit Mechanisms," Psychological Review, 37, 1930, p. 515.
} 
the movements of the fingers which often occur when one is asked to describe how one buttons his coat, such movements serving solely to help elicit the verbal responses given in reply to the question. Such acts were regarded as "an organic, physiological-strictly internal and individual-symbolism," "not to be confused with the purely stimulus acts of social communication." ${ }^{40}$ They were regarded as "the organic basis of symbolism" ${ }^{41}$ and often equated with "ideas" since they provided a reaction to "the not here and now."

In these early articles Hull generally equates 'symbolic act' and 'purestimulus act.' Our purpose is to show that pure-stimulus acts are not in themselves necessarily signs, but that they occur in all symbol-processes and may themselves become signs of an especially important kind.

Take the case of the finger motions made in response to the question as to how one buttons his coat. These motions may help to elicit a verbal description of how the coat is buttoned, but in terms of our previous definition of 'sign,' it is hard to see the sense in which they are themselves signs; they do not determine behavior with respect to buttoning a coat nor does the fact that they operate causally in helping to produce verbal behavior make them a sign of such behavior. If this example is doubtful, because of its complexity and the intrusion of language signs, certainly there are many cases of animal behavior in which there are chains of responses such that the making of one response provides the stimulus for another response, and yet in which normal usage would not say that such pure-stimulus acts are signs of the succeeding, or the terminal, responses. A pure-stimulus act is not, then, in itself necessarily a sign.

Nevertheless, on the definition of sign which has been advocated, a symbol-process always involves a pure-stimulus act. For on that definition the symbol is a response (or product of a response) of the organism for which it is a sign, that is, the stimulus to an interpretant in a symbol-process is itself a pure-stimulus act (though it is seldom "pure" in the sense that this is its sole function). Hence such acts,

${ }^{4}$ Ibid., p. $517 \mathrm{n}$.

"Ibid., p. 524 . Similar views were expressed in "Goal Attraction and Directing Ideas Conceived as Habit Phenomena," ibid., 1931, pp. 487-506, in Hypnosis and Suggestibility (1933), and in "The Mechanism of the Assembly of Behavior Segments in Novel Combinations Suitable for Problem Solution," Psychological Review, 42, 1935, pp. 219-245. 
though not in themselves necessarily signs, are components of all symbol-processes. Thus a response which is a substitute for the sound of the buzzer is, if the buzzer is a sign, a pure-stimulus act which serves as a factor determining the reponse to the situation in which it appears. Hence the pure-stimulus act, though not the organic basis of all signprocesses, is an essential organic basis of symbol-processes.

This analysis also seems to clarify the relation within symbol-processes of pure-stimulus acts and antedating responses-a point left in some uncertainty in Hull's formulations. An antedating response is defined by Hull as a response which appears temporarily prior to the appearance of the stimulus which normally evokes the response. If only a portion of the response appears-such as in the making of masticatory movements as a food object is approached-the response is said by Hull to be a fractional antedating response. It is interesting that in Hull's 1937 miniature theoretical system, ${ }^{42}$ the term 'pure-stimulus act' does not appear, though the term 'antedating' (or 'anticipatory') response is used to explain the same phenomena dealt with in his earlier articles. It seems clear, however, that 'pure-stimulus act' is the more basic term, and that while antedating responses (as distinct from preparatory sets) occur in many, or perhaps all, sign-processes, they are not essential to, nor sufficient for, the definition of such processes.

The pure-stimulus act in a sign-process might of course be an antedating response, but it need not be. And the interpretant, for which the pure-stimulus act is a preparatory-stimulus, need not, on the proposed definition, appear in the form of an antedating response (the reader of a letter about China need not respond as he would if he were in the presence of the events described). Nevertheless, it is understandable that in many cases, and perhaps in all, the interpretant should to some degree appear as a fractional antedating response: the preparatory-stimulus may, but need not, evoke antedating responses. A preparatory set may exist without there being antedating responses. Fractional antedating responses may, when they occur, be used as part of the evidence for determining the existence of sign-processes and for determining what is signified in a sign-process. But since such responses may occur

a "Mind, Mechanism and Adaptive Behavior," Psychological Review, 44, 1937, pp. 1-32. 
without there being a sign-process, and sign-processes without there necessarily being such a response (or such a response detectable by existing methods), sign-processes are not formulable in terms of antedating responses.

Hence our conclusion that Hull has identified important factors in symbol-processes (the pure-stimulus act as a necessary component, and fractional antedating responses as frequent components) but that these factors do not in themselves provide an adequate account of sign-behavior. When embodied into our account, however, they help elucidate the mechanism of such behavior. It is to the work of such experimenters as Hull and his colleagues that we can confidently look for the further elucidation of the detailed mechanisms of sign-behavior and its relation to the general processes of behavior. And since this group has consistently stood for the point of view which stresses the continuity of human and sub-human behavior, it is to be hoped that they will not choose to restrict sign-behavior to human language behavior and thus obscure the relation of the more complex forms of sign-behavior to the simpler forms of such behavior out of which language signs develop and which they presuppose.

\section{The Uniqueness of Sign-Behavior}

The preceding pages have endeavored to formulate sign-behavior as a special and distinctive phenomenon, and yet to show that the factors which enter into it have already been isolated to some extent in the studies of behavior. The uniqueness of sign-processes lies, in short, in the pattern of their constituents, and not in the constituents themselves. Nothing but the usual terms of stimulus and response are involved, set of course within the framework of behavior-families. There is nothing unusual about the stimuli except that in a sign-process something becomes an evocative stimulus only because of the existence of something else as a preparatory-stimulus-but this is itself a special case of the wellknown fact that stimuli evoke responses only under certain conditions. And the responses involved are responses which may occur outside of sign-processes; thus the response of going to food may be, but need not be, conditional upon the appearance of a preparatory-stimulus, and a 
response to the sound of a buzzer may be, but need not be, a purestimulus act which serves as a symbol. Stimuli which are signs in one situation may not be signs in another situation, and responses which are components of sign-processes may at other times occur outside of such processes.

In a similar way the description of sign-processes cuts across the more traditional categories of behavior theory. Nothing in the formulation of signs makes it impossible for there to be unlearned signs; whether there are or are not is an empirical problem. In those cases where signs are acquired in the life time of the individual, the question may be raised whether they are instances of "conditioning." Tolman, as we have seen, has suggested that for behavior theory the concept of sign is more basic than that of conditioning, while Hull has attempted to explain Tolman's results in terms of the mechanisms of conditioning. Such differences are largely due to the great vagueness in the term 'conditioned response'; the preceding discussion of the views of Tolman and Hull has aimed to show, at least with respect to signbehavior, that there is nothing incompatible in their positions. As to the general issue, it can be said that if 'conditioning' is used in its earlier narrow senses, sign-behavior, even when learned, is not an instance of conditioning since it is not reducible to the case of a response which was previously made to one stimulus now being made in an identical manner to another stimulus. If, however, the term 'conditioning' be extended, as Skinner has done, to cases of "pseudo-reflexes," that is, cases where responses occur in the presence of objects which are not strictly speaking stimuli which by themselves evoke those responses, ${ }^{43}$ then learned sign-behavior may be regarded as a case of conditioning. But in this case it is essential to distinguish the cases of conditioning in which signs occur from those cases in which signs do not occur. Whether or not, then, learned sign-behavior is or is not conditioned behavior is in part a matter of definition, and not so important as the study of the actual mechanisms involved in sign-behavior and the relation of such behavior to other forms of behavior.

${ }^{\text {ts }}$ B. F. Skinner, The Behavior of Organisms (1938). Skinner calls such responses which are not elicited by specific environmental stimuli operant behavior, as contrasted to respondent behavior. In his terms our definition of sign could presurmably be stated as follows: a sign is a stimulus to behavior which is a pseudo-reflex in relation to some feature of the environment. 
The situation is analogous with respect to the category of habit. If the term 'habit,' applied to sign-behavior, means that an animal frequently interprets certain events in a similar way, then much signbehavior is habitual. But it need not be: it is quite possible for the signification of an event to vary from situation to situation, and for what is signified to be signified by different signs in different situations. An example would be the case where an animal observes food put in a black box which is then, outside his field of observation, shuffled with boxes of other colors; under such situations, the black box may be a sign of food, while in the next repetition of the experiment if the food is put in a yellow box, the yellow box may become a sign of food and the black box lose in that situation its sign status. ${ }^{44}$

The use of the term 'sign' by behavior theorists of various schools underlines the importance of attaining clarity and agreement as to the signification of this and related terms. It is believed that the preceding analyses have contributed to this end. The problems of the specific mechanisms of sign-behavior, of the comparison of such behavior in various kinds of animals, of the evolutionary history of sign-processes, and of the relation of sign-behavior to behavior in general then become problems common to all students of behavior, and problems capable of empirical formulation and experimental resolution.

\footnotetext{
"See Robert Yerkes and Henry W. Nissen, "Pre-Linguistic Sign Behavior in Chimpanzee," Science, $89,1939,585-87$. The authors specifically stress the labile character of such behavior and speak of "symbols" to differentiate it from "signs" which have a more habitual character. We do not follow this terminological usage.
} 


\section{BIBLIOGRAPHY}

\section{Books and Articles}

Acton, H. B. "The Expletive Theory of Morals," Analysis, Vol. 4 (1936), pp. $42-45$.

Adler, Mortimer. How to Read a Book. New York: Simon and Schuster, 1940.

Ajdukiewicz, Kasimir. "Sprache und Sinn," Erkenntnis, Vol. 4 (1934), pp. 100-38.

- "Das Weltbild und die Begriffsapparatur," Erkenntnis, Vol. 4 (1934), pp. 259-87.

Aldrich, Virgil C. "Symbolization and Similarity," The Monist, Vol. 42 (1932), pp. 564-76.

$57-71$.

Allport, F. H. Social Psychology. Boston: Houghton Mifflin Company, 1924.

Allport, Gordon W., and Vernon, Philip E. Studies in Expressive Movement. New York: The Macmillan Company, 1933.

Ames, Van Meter. "The Novel: Between Art and Science," Kenyon Review, Vol. 5 (1943), pp. 34-48.

- "Art and Science," Kenyon Review, Vol. 6 (1944), pp. 101-13.

Angyal, Andras. Foundations for a Science of Personality. New York: The Commonwealth Fund, 1941.

Arnheim, Rudolf. Experimentell-psychologische Untersuchungen zum Ausdrucksproblem. Berlin: Springer, 1928.

Arnold, Thurman W. The Symbols of Government. New Haven: Yale University Press, 1935.

- The Folklore of Capitalism. New Haven: Yale University Press, 1937.

Arréat, L. "Signes et symboles," Revue philosophique, Vol. 75 (1913), pp. 51-70.

Ayer, A. J. "Demonstration of the Impossibility of Metaphysics," Mind, Vol. 43 (1934), pp. 335-45.

1936. 
Ayer, A. J., Whiteley, C. H., and Black, M. "Truth by Convention: A Symposium," Analysis, Vol. 4 (1936), pp. 28-32.

Balken, Eva Ruth. "Psychological Researches in Schizophrenic Language and Thought," The Journal of Psychology, Vol. 16 (1943), pp. 153-76.

- "A Delineation of Schizophrenic Language and Thought in a Test of Imagination," The Journal of Psychology, Vol. 16 (1943), pp. 239-71.

Balken, Eva Ruth, and Masserman, Jules H. "The Language of Phantasy: III. The Language of the Phantasies of Patients with Conversion Hysteria, Anxiety State, and Obsessive-Compulsive Neuroses," Journal of Psychology, Vol. 10 (1940), pp. 75-86. (See Jules H. Masserman for articles I and II on Phantasy.)

Bally, Ch. Le langage et la vie. Genève: Edition Atar, 1913.

"La contrainte sociale dans le langage," Revue internationale de sociologie, Vol. 35 (1927), pp. 209-29.

- "Les notions grammaticales d'absolu et de relatif," Journal de psychologie, Vol. 30 (1933), pp. 341-54.

Bartlett, F. C. "The Social Functions of Symbols," Australian Journal of Psychology and Philosophy, Vol. 3 (1925), pp. 1-11.

Bartlett, George R. "Criteria for the Sign 'God' in Empirical Religious Inquiry," Ph.D. Dissertation, University of Chicago, 1942.

Baudouin, Charles. Psychoanalysis and Aesthetics. Translated by E. and C. Paul. New York: Dodd, Mead and Company, 1924.

Baylis, Charles A. "Meanings and Their Exemplifications," Journal of Philosophy, Vol. 27 (1930), pp. 169-74.

- "Critical Comments on the 'Symposium on Meaning and Truth," Philosophy and Phenomenological Research, Vol. 5 (1944), pp. 80-93.

Becker, O. "Das Symbolische in der Mathematik," Blätter für deutsche Philosophie, Vol. 1 (1928), pp. 329-48.

Benjamin, A. Cornelius. "Outlines of an Empirical Theory of Meaning," Philosophy of Science, Vol. 3 (1936), pp. 250-66.

-The Logical Structure of Science. London: K. Paul, Trench, Trubner \& Co., Ltd., 1936.

- An Introduction to the Philosophy of Science. New York: The Macmillan Company, 1937.

- "The Operational Theory of Meaning," The Philosophical Review, Vol. 46 (1937), pp. 644-49.

- "Science and Vagueness," Philosophy of Science, Vol. 6 (1939), pp. $422-31$.

- "What Is Empirical Philosophy?" Journal of Philosophy, Vol. 36 (1939), pp. 517-25. 
Benne, K. D., Axtelle, George E., Smith, B. O., and Raup, R. Bruce. The Discipline of Practical Judgment in a Democratic Society. Yearbook, National Society of College Teachers of Education, Vol. 28. Ann Arbor: Ann Arbor Press, 1943.

Bentham, Jeremy. "Linguistic Fictions," Psyche, Vol. 9 (1928), pp. 15-24. Bentley, Arthur F. Linguistic Analysis of Mathematics. Bloomington, Indiana: The Principia Press, 1932.

-. "On a Certain Vagueness in Logic, I," Journal of Philosophy, Vol. 42 (1945), pp. 6-27.

- "On a Certain Vagueness in Logic, II," Journal of Philosophy, Vol. 42 (1945), pp. 39-51.

Bentley, M., and Varon, E. J. "An Accessory Study of 'Phonetic Symbolism," American Journal of Psychology, Vol. 45 (1933), pp. 76-86.

Bergmann, Gustav. "Pure Semantics, Sentences, and Propositions," Mind, Vol. 53 (1944), pp. 238-57.

Bevan, Edwyn. Symbolism and Belief. New York: The Macmillan Company, 1938.

Bierens de Haan, J. A. "Langue humaine; langage animal," Scientia, Vol. 55 (1934), pp. 40-49.

Black, Max. "The Principle of Verifiability," Analysis, Vol. 2 (1934), pp. 1-6.

- "Vagueness," Philosophy of Science, Vol. 4 (1937), pp. 427-55.

-1. "Conventionalism in Geometry and the Interpretation of Necessary Statements," Philosophy of Science, Vol. 9 (1942), pp. 335-49.

Bloomfield, Leonard. "A Set of Postulates for the Science of Language," Language, Vol. 2 (1926), pp. 153-64.

-. "Linguistics as a Science," Studies in Philology, Vol. 27 (1930), pp. 553-57.

- Language. New York: H. Holt and Company, 1933.

- "Linguistic Aspects of Science," Philosophy of Science, Vol. 2 (1935), pp. 499-517.

- "Language or Ideas?" Language, Vol. 12 (1936), pp. 89-95.

- Linguistic Aspects of Science. International Encyclopedia of Unified Science, Vol. 1, No. 4. Chicago: University of Chicago Press, 1939.

Boas, Franz. Handbook of American Indian Languages. Pt. 1. Washington: Government Printing Office, 1911.

- Race, Language and Culture. New York: The Macmillan Company, 1940.

Boder, David P. "The Adjective-Verb Quotient; A Contribution to the Psychology of Language," The Psychological Record, Vol. 3 (1940), pp. 309-44. 
Bodmer, Frederick. The Loom of Language. Edited and Arranged by Lancelot Hogben. London: G. Allen \& Unwin, Ltd., 1943.

Bogoslovsky, Boris. The Technique of Controversy. New York: Harcourt, Brace and Company, 1928.

Bohnert, H. G. "The Semiotic Status of Commands," Philosophy of Science, Vol. 12, 1945.

Boyer, Merle W. "Sign Analysis as a Method for Philosophy of Religion," Ph.D. Dissertation, University of Chicago, 1941.

Bréal, Michel. Essai de sémantique (science des significations). 5. éd.; Paris: Hachette, 1911. (First published in 1897.)

Bréhier, Emile. "Origine des images symboliques," Revue philosophique, Vol. 75 (1913), pp. 135-55.

Brewster, John M. "A Behavioristic Account of the Logical Function of Universals," Journal of Philosophy, Vol. 33 (1936), pp. 505-14; 533-17. Bridgman, P. W. Logic of Modern Physics. New York: The Macmillan Company, 1927.

- The Nature of Physical Theory. Princeton: Princeton University Press, 1936.

- The Intelligent Individual and Society. New York: The Macmillan Company, 1938.

- "Operational Analysis," Philosophy of Science, Vol. 5 (1938), pp. 114-31.

Brill; A. A. "The Universality of Symbols," The Psychoanalytic Review, Vol. 30 (1943), pp. 1-18.

Britton, Karl. "Empirical Foundation for Logic," Analysis, Vol. 2 (1934), pp. 37-42.

- "Language: Public and Private," The Monist, Vol. 45 (1935), pp. $1-59$.

- "The Truth of Religious Propositions," Analysis, Vol. 3 (1935), pp. 21-27.

- Communication; a Philosophical Study of Language. International Library of Psychology, Philosophy, and Scientific Method. New York: Harcourt, Brace and Company, 1939.

Brondal, Viggo. Les Parties du discours. Copenhagen: G. E. C. Gad, 1928.

- "L'Autonomie de la syntaxe," Journal de psychologie, Vol. 30 (1933), pp. 217-24.

Brown, Harold Chapman. "Language and the Associative Reflex," Journal of Philosophy, Vol. 13 (1916), pp. 645-49.

Brunswik, Egon. "Psychology as a Science of Objective Relations," Philosophy of Science, Vol. 4 (1937), pp. $227-60$. 
. "Probability as a Determiner of Rat Behavior," Journal of Experimental Psychology, Vol. 25 (1939), pp. 175-97.

- "Thing Constancy as Measured by Correlation Coefficients," Psychological Review, Vol. 47 (1940), pp. 69-78.

Brunswik, Egon, Hull, Clark L., and Lewin, Kurt. "Symposium on Psychology and Scientific Method," Psychological Review, Vol. 50 (1943), pp. 255-310.

Buchanan, Scott. Symbolic Distance in Relation to Analogy and Fiction. London: K. Paul, Trench, Trubner \& Co., 1932.

- The Doctrine of Signatures. New York: Harcourt, Brace and Company, 1938.

Buchler, Justus. "Value-Statements," Analysis, Vol. 4 (1937), pp. 49-58.

Bühler, Charlotte. Kindheit und Jugend. Leipzig: S. Hirzel, 1928.

Bühler, Karl. Die geistige Entwicklung des Kindes. Jena: G. Fischer, 1921.

- Die Krise der Psychologie. Jena: G. Fischer, 1927. 2d ed. 1929.

- Ansdruckstheorie; Das System und Geschichte aufgezeigt. Jena: G. Fischer, 1933.

- Sprachtheorie. Jena: G. Fischer, 1934.

Burke, Kenneth. Permanence and Change. New York: New Republic, Inc., 1935.

- "Semantic and Poetic Meaning," Southern Review, Vol. 4 (1939), pp. 501-23.

- The Philosophy of Literary Form. Baton Rouge, Louisiana: State University Press, 1941.

- A Grammar of Motives. New York: Prentice-Hall, Inc., 1945.

Burrow, Trigant. "Behavior Mechanisms and Their Phylopathology," The Psychoanalytic Review, Vol. 22 (1935), pp. 169-81.

Cameron, Norman. "Reasoning, Regression and Communication in Schizophrenics," Psychological Review. Monograph Supplements, Vol. 50 (1938), pp. 1-34.

Campbell, Douglas Gordon. "General Semantics. Implications of Linguistic Revision for Theoretical and Clinical Neuro-Psychiatry," American Journal of Psychiatry, Vol. 93 (1937), pp. 789-807.

Carmichael, R. D. "Meaning in the Case of Mathematical Postulates," The Monist, Vol. 35 (1925), pp. 372-404.

Carnap, Rudolf. Physikalische Begrifsbildung. Karlsruhe: G. Braun, 1926. - "Eigentliche und uneigentliche Begriffe," Symposion, Vol. 1 (1927), pp. 355.75.

-Psychologie in physikalischer Sprache," Erkenntnis, Vol. 3 (1932), pp. 107-42. 
The Unity of Science. Psyche Miniatures, General Series No. 63. London: K. Paul, Trench, Trubner \& Co., Ltd., 1934.

-. Philosophy and Logical Syntax. London: K. Paul, Trench, Trubner \& Co., Ltd., 1935.

- "Les Concepts psychologiques et les concepts physiques sont-ils foncièrement différents?" Revue de synthèse, Vol. 10 (1935), pp. 43-53. - "Formalwissenschaft und Realwissenschaft," Erkenntnis, Vol. 5 (1935), pp. 30-36.

- "Testability and Meaning," Philosophy of Science, Vol. 3 (1936), pp. 419-71; Vol. 4 (1937), pp. 1-40.

- The Logical Syntax of Language. New York: Harcourt, Brace and Company, 1937.

- "Logical Foundations of the Unity of Science," International Encyclopedia of Unified Science, Vol. 1, No. 1. Chicago: The University of Chicago Press, 1938. Pp. 42-62.

- Foundations of Logic and Mathematics. International Encyclopedia of Unified Science, Vol. 1, No. 3. Chicago: University of Chicago Press, 1939.

- Introduction to Semantics. Cambridge: Harvard University Press, 1942.

- Formalization of Logic. Cambridge: Harvard University Press, 1943.

- "The Two Concepts of Probability," Philosophy and Phenomenological Research, Vol. 5 (1945), pp. 513-32.

$72-97$.

-.72. "On Inductive Logic," Philosophy of Science, Vol. 12 (1945), pp.

Carpenter, C. R., and Locke, N. M. "Notes on Symbolic Behavior in a Cebus Monkey," The Journal of Genetic Psychology, Vol. 51 (1937), pp. 267-78.

Carroll, John B. "The Analysis of Verbal Behavior," Psychological Review, Vol. 51 (1944), pp. 102-19.

Casamajor, J. "The Evolution of Symbols in the Development of Consciousness," Archives of Neurology and Psychiatry, Vol. 22 (1929), pp. $865-72$.

Cason, Hulsey. "General Aspects of the Conditioned Response," Psychological Review, Vol. 32 (1925), pp. 298-316.

- "The Role of Verbal Activities in the Conditioning of Human Subjects," Psychological Review, Vol. 41 (1934), pp. 563-71.

Cassirer, Ernst. Die Begriffstorm im mythischen Denken. Leipzig: B. G. Teubner, 1922.

- Philosophie der symbolischen Formen. Berlin: B. Cassirer, 1923-31. 
"Das Symbolproblem und seine Stellung im System der Philosophie," Zeitschrift für Äesthetik, Vol. 21 (1927), pp. 295-312.

- "Etude sur la pathologie de la conscience symbolique," Journal de psychologie, Vol. 26 (1929), pp. 289-336; 523-66.

- "Die Sprache und der Aufbau der Gegenstandswelt," Bericht über den Kongress der deutschen Gesellschaft für Psychologie, Vol. 12 (1932), pp. 134-45.

- "Zur Logik des Symbolbegriffs," Theoria, Vol. 4 (1938), pp. 145-75.

- An Essay on Man. New Haven: Yale University Press, 1944.

- Language and Myth. Trans. by Susanne K. Langer. New York: Harper and Brothers, 1946.

Chapple, Eliot Dismore, and Coon, Carleton Stevens. Principles of Anthropology. New York: H. Holt and Company, 1942.

Chase, Stuart. The Tyranny of Words. New York: Harcourt, Brace and Company, 1938.

Clayton, Alfred S. Emergent Mind and Education; $A$ Study of George $H$. Mead's Bio-Social Behaviorism from an Educational Point of View. New York: Teachers College, Columbia University, 1943.

Cocchiara, Giuseppe. Il Linguaggio del gesto. Torino: Fratelli Bocca, 1932.

Coghill, George E. "The Neuro-embryologic Study of Behavior: Principles, Perspective and Aim," Science, Vol. 78 (1933), pp. 131-38.

Cohen, Morris. "Concepts and Twilight Zones," Journal of Philosophy, Vol. 24 (1927), pp. 673-83.

Cooley, Charles Horton. Human Nature and the Social Order. Rev. ed.; New York: C. Scribner's Sons, 1922.

Cooley, John C. A Primer of Formal Logic. New York: The Macmillan Company, 1942.

Copilowish, Irving M. "Border-Line Cases, Vagueness, and Ambiguity," Philosophy of Science, Vol. 6 (1939), pp. 181-95.

Couturat, Louis. La Logique de Leibniz d'après des documents inédits. Paris: F. Alcan, 1901.

Cowles, J. T., and Nissen, H. W. "Reward-Expectancy in Delayed Responses of Chimpanzees," Journal of Comparative Psychology, Vol. 24 (1937), pp. 345-58.

Crawford, M. P. "The Cooperative Solving of Problems by Young Chimpanzees," Comparative Psychology Monographs, Vol. 14 (1937). Pp. 88.

Creel, Herrlee Glessner. "On the Nature of Chinese Ideography," T'oung Pao, Vol. 32 (1936), pp. 85-161.

Croce, Benedetto. Aesthetic as Science of Expression and General Linguistic. Translated by Douglas Ainslie. $2 \mathrm{~d}$ ed. London: Macmillan and Co., Ltd., 1922. (First published in Italian in 1902.) 
Cunningham, G. Watts. "Perspective and Context in the Meaning-Situation," University of California Publications in Philosophy, Vol. 16, No. 2. Berkeley, Calif.: University of California Press, 1935. Pp. $29-52$.

-. "Meaning, Reference, and Significance," Philosophical Review, Vol. 47 (1938), pp. 155-75.

- "On the Linguistic Meaning-Situation," Philosophy and Phenomenological Research, Vol. 4 (1943), pp. 251-65.

Daiches, David. The Place of Meaning in Poetry. London: Oliver and Boyd, 1935.

Dalkey, Norman C. "The Plurality of Language Structures." Ph.D. Dissertation. Los Angeles: University of California, 1942.

- "The Limits of Meaning," Philosophy and Phenomenological Research, Vol. 4 (1944), pp. 401-409.

DeGroot, A. W. "Zur Grundlegung der Morphologie und der Syntax," Algemeen Nederlands Tijdschrift voor Wijsbegeerte en Psychologie, Vol. 32 (1938), pp. 145-74.

DeLacy, Estelle A., and Phillip H. Philodemus. Philadelphia: Philological Monographs, 1941.

De Laguna, Grace Andrus. Speech, Its Function and Development. New Haven: Yale University Press, 1927.

Dewey, John. Essays in Experimental Logic. Chicago: University of Chicago Press, 1916.

- "Knowledge and Speech Reaction," Journal of Philosophy, Vol. 19 (1922), pp. 561-70.

- Philosophy and Civilization. New York: Minton, Balch \& Company, 1931.

- Art as Experience. New York: Minton, Balch \& Company, 1934. . Logic, the Theory of Inquiry. New York: H. Holt and Company, 1938.

Theory of Valuation. International Encyclopedia of Unified Science, Vol. 2, No. 4. Chicago: University of Chicago Press, 1939.

- "Peirce's Theory of Linguistic Signs, Thought, and Meaning," Journal of Philosophy, Vol. 43 (1946), pp. 85-95.

Dewey, John, and Bentley, Arthur F. "A Terminology for Knowings and Knowns," Journal of Philosophy, Vol. 42 (1945), pp. 225-47.

Ducasse, C. J. "Verification, Verifiability, and Meaningfulness," Journal of Philosophy, Vol. 33 (1936), pp. 230-36.

- "Symbols, Signs, and Signals," Journal of Symbolic Logic, Vol. 4 (1939), pp. 41-52.

- "Propositions, Opinions, Sentences, and Facts," The Journal of Philosophy, Vol. 37 (1940), pp. 701-11.

-. Philosophy as a Science. New York: O. Piest, 1941. 
- "Some Comments on C. W. Morris's 'Foundations of the Theory of Signs," "Philosophy and Phenomenological Research, Vol. 3 (1942), pp. 43-52.

- "Propositions, Truth, and the Ultimate Criterion of Truth," Philosophy and Phenomenological Research, Vol. 4 (1944), pp. 317-40.

Durkheim, Emile. "Représentations individuelles et représentations collectives," Revue de métaphysique et de morale, Vol. 6 (1898), pp. 273-302.

- The Elementary Forms of the Religious Life. Translated by Joseph Ward Swain. London: G. Allen \& Unwin, Ltd., 1915. (First published in French in 1912.)

Eaton, Ralph Monroe. Symbolism and Truth. Cambridge: Harvard University Press, 1925.

Efron, David. Gesture and Environment. New York: King's Crown Press, 1941.

Eisenson, Jon. The Psychology of Speech. New York: F. S. Crofts \& Co., 1938.

Emerson, Alfred Edward. "Communication among Termites," Fourth International Congress of Entomology, Vol. 2 (1929), pp. 722-27.

- "Termite Nests, A Study of the Phylogeny of Behavior," Ecological Monographs, Vol. 8 (1938), pp. 247-84.

- "Biological Sociology," Denison University Bulletin, Journal of the Scientific Laboratories, Vol. 36 (1941), pp. 146-55.

- "Basic Comparisons of Human and Insect Societies," Biological Symposia, Vol. 8 (1942), pp. 163-76.

Empson, William. Seven Types of Ambiguity. London: Chatto and Windus, 1930.

- "The Need for 'Translation' Theory in Linguistics," Psyche, Vol. 15 (1935), pp. 188-97.

Endres, Franz Carl. "Vom Wesen des Symbols," Psychologische Rundschau, Vol. 3 (1931), pp. 108-16; 146-50.

Erdmann, Karl Otto. Die Bedeutung des Wortes. Leipzig: E. Avenarius, 1900.

Esper, E. A. "Language," $A$ Handbook of Social Psychology. Worcester, Mass.: Clark University Press, 1935. Pp. 417-60.

Feigl, Herbert. "Logical Empiricism," Twentieth Century Philosophy: Living Schools of Thought. Edited by Dagobert D. Runes. New York: Philosophical Library, 1943. Pp. 373-416.

Ferrero, Guglielmo. Les Lois psychologiques du symbolisme. Paris: F. Alcan, 1895.

Fite, Warner. "The Philosopher and His Words," Philosophical Review, Vol. 44 (1935), pp. 120-37. 
Fox, Ch. W. "An Experimental Study of Naming," American Journal of Psychology, Vol. 47 (1935), pp. 545-79.

Frank, Jerome. Law and the Modern Mind. New York: Brentano's, 1930. Frank, Lawrence K. "What is Social Order?" The American Journal of Sociology, Vol. 49 (1944), pp. 470-77.

Frank, Philipp. Interpretations and Misinterpretations of Modern Physics. Paris: Hermann \& $\mathrm{C}^{\mathrm{ie}}, 1938$.

- Between Physics and Philosophy. Cambridge, Mass.: Harvard University Press, 1941.

- Foundations of Physics. International Encyclopedia of Unified Science, Vol. 1, No. 7. Chicago: University of Chicago Press, 1946.

Frege, B. "Über Sinn und Bedeutung," Zeitschrift für Philosophie, Vol. 100 (1891), pp. 25-50.

French, Thomas M. "An Analysis of the Goal Concept Based upon Study of Reactions to Frustration," The Psychoanalytic Review, Vol. 28 (1941), pp. 61-71.

Frenkel-Brunswik, Else. "Motivation and Behavior," Genetic Psychology Monographs, Vol. 26 (1942), pp. 121-265. Also published, Motivation and Behavior. Provincetown, Mass.: The Journal Press, 1942.

Freud, Sigmund. The Interpretation of Dreams. Translated by A. A. Brill. New York: The Macmillan Co., 1915.

- Introductory Lectures on Psychoanalysis. Translated by Joan Riviere. London: G. Allen \& Unwin, Ltd., 1922.

- New Introductory Lectures on Psychoanalysis. Translated by W. J. H. Sprott. New York: W. W. Norton \& Company, Inc., 1933.

Fries, Horace S. "On an Empirical Criterion of Meaning," Philosophy of Science, Vol. 3 (1936), pp. 143-51.

Furley, Paul Hanly. "Conscious and Unconscious Factors in Symbolism," Psychological Monographs, Vol. 27 (1919), pp. 349-86.

Galt, William. "Our Mother Tongue," The Psychoanalytic Review, Vol. 30 (1943), pp. 241-62.

Gantt, W. Horsley. "An Experimental Approach to Psychiatry," American Journal of Psychiatry, Vol. 92 (1936), pp. 1007-21.

- Experimental Basis for Neurotic Behavior. New York: P. B. Hoeber, Inc., 1944.

Gardiner, Alan H. The Theory of Speech and Language. Oxford: The Clarendon Press, 1932.

Gätschenberger, Richard. Grundzüge einer Psychologie des Zeichens. Regensburg: G. J. Manz, 1901.

i. B.: G. Braun, 1920. 

1932.

Zeichen, die Fundamente des Wissens. Stuttgart: Frommann,

Geller, A., Kaplan, D., and Lasswell, Harold D. "An Experimental Comparison of Four Ways of Coding Editorial Content," Journalism Quarterly, Vol. 19, No. 4, December, 1942.

Gentry, George V. "Some Comments on Morris's 'Class' Conception of the Designatum,” Journal of Philosophy, Vol. 41 (1944), pp. 376-84.

Gilman, B. I. "Report on an Experimental Test of Musical Expressiveness," American Journal of Psychology, Vol. 4 (1892), pp. 558-76.

Goldstein, K. "L'Analyse de l'aphasie et l'étude de l'essence du langage," Journal de psychologie, Vol. 30 (1933), pp. 430-96.

Gomperz, Heinrich. Über Sinn und Sinngebilde, Verstehen und Erklären. Tübingen: Mohr, 1929.

- "The Meanings of "Meaning," Philosophy of Science, Vol. 8 (1941), pp. 157-83.

Grandjean, Frank. "La Contrainte sociale et la logique," Revue internationale de sociologie, Vol. 37 (1929), pp. 113-41.

Graves, Harold F., and Bowman, John S. Types of Persuasion. New York: The Cordon Company, 1938.

Grene, Marjorie. "Theories of Interpretation in the Law of Contracts," The University of Chicago Law Review, Vol. 6 (1939), pp. 374-98.

Grudin, Louis. A Primer of Aesthetics: Logical Approaches to a Philosophy of Art. New York: Covici, Friede, 1930.

Hahn, Hans. Logik, Mathematik und Naturerkennen. Wien: Verlag Gerold \& Co., 1933.

Hahn, Lewis Edwin. A Contextualistic Theory of Perception. Los Angeles: University of California Press, 1942.

Hall, Everett W. 'Some Meanings of Meaning in Dewey's Experience and Nature," Journal of Philosophy, Vol. 25 (1928), pp. 169-81.

. "The Meaning of Meaning in Hollingworth's The Psychology of Thought," Journal of Philosophy, Vol. 25 (1928), pp. 393-403.

. "The Extra-Linguistic Reference of Language," Mind, Vol. 52 (1943), pp. 230-46.

- "Metaphysics," Twentieth Century Philosophy. Edited by D. D. Runes. New York: Philosophical Library, 1943.

Hamann, Richard. Das Symbol. Gräfenhainichen: W. Hecker, 1902.

Handman, Max. "War, Economic Motives, and Economic Symbols," American Journal of Sociology, Vol. 44 (1939), pp. 629-48.

Hawley, Frank. "Verbal Forms and Functions," Psyche, Vol. 8 (1928), pp. 3-17.

Hayakawa, S. I. Language in Action. New York: Harcourt, Brace and Company, 1941. 
- "Science and Sanity," American Speech, Vol. 18 (1943), pp. 219-26. Reprinted as "Linguistics and the Future" in Etc., A Review of General Semantics, Vol. 1 (1944), pp. 148-53.

Head, Henry. "Aphasia and Kindred Disorders of Speech," Brain, Vol. 43 (1920), 87-165.

- "Disorders of Symbolic Thinking and Expression," British Journal of Psychology, Vol. 11 (1921), pp. 179-93.

Heider, Fritz. "Social Perception and Phenomenal Causality," Psychological Review, Vol. 51 (1944), pp. 358-74.

Hempel, Carl G. "Analyse logique de la psychologie," Revue de synthèse, Vol. 10 (1935), pp. $27-42$.

\section{3-80.}

Henry, Jules. "The Linguistic Expression of Emotion," American Anthropologist, Vol. 38 (1936), pp. $250-56$.

Herschberger, Ruth. "The Structure of Metaphor," Kenyon Review, Vol. 5 (1943), pp. 433-43.

Hevner, Kate. "Expression in Music," Psychological Review, Vol. 42 (1935), pp. 186-204.

- "Experimental Studies of the Elements of Expression in Music," American Journal of Psychology, Vol. 48 (1936), pp. 246-68.

- . "An Experimental Study of the Affective Value of Sounds in Poetry," American Journal of Psychology, Vol. 49 (1937), pp. 419-34.

Hexner, Erwin. Studies in Legal Terminology. Chapel Hill: The University of North Carolina Press, 1941.

Heyl, Bernard C. New Bearings in Esthetics and Art Criticism; a Study in Semantics and Evaluation. New Haven: Yale University Press, 1943.

Higginson, Glenn D. "The Place of Meaning in Psychology," Psychological Review, Vol. 44 (1937), pp. 491-504.

Hilgard, Ernest R., and Marquis, Donald G. Conditioning and Learning. New York: D. Appleton-Century Company, Inc., 1940.

Hinshaw, Virgil G., Jr. "The Epistemological Relevance of Mannheim's Sociology of Knowledge," The Journal of Philosophy, Vol. 40 (1943), pp. $57-72$.

- "The Pragmatist Theory of Truth," Philosophy of Science, Vol. 11 (1944), pp. 82-92.

Hinton, Norman H. Political Semantics. A Case Study. Ph.D. Dissertation, Columbia University, 1941.

Hodges, H. A. Wilhelm Dilthey: An Introduction. London: K. Paul, Trench, Trubner and Co., Ltd., 1944.

Hofstadter, Albert. "On Semantic Problems," Journal of Philosophy, Vol. 35 (1938), pp. 225-32. 
- "Subjective Teleology," Philosophy and Phenomenological Research, Vol. 2 (1941), pp. 88-97.

- "Objective Teleology," The Journal of Philosophy, Vol. 38 (1941), pp. 29-39.

Hofstadter, Albert, and McKinsey, J. C. C. "On the Logic of Imperatives," Philosophy of Science, Vol. 6 (1939), pp. 446-57.

Hollingworth, H. L. "Meaning and the Psycho-physical Continuum," Journal of Philosophy, Vol. 20 (1923), pp. 433-41.

- "Symbolic Relations in Thinking," Journal of Philosophy, Vol. 20 (1923), pp. 516-24.

- "Particular Features of Meaning," Psychological Review, Vol. 31 (1924), pp. 348-68.

- Abnormal Psychology. New York: The Ronald Press Company, 1930.

Holloway, Owen E. "Grammar and Notation," Psyche, Vol. 16 (1936), pp. 100-21.

Horn, Ernest. "Language and Meaning," The Psychology of Learning 1942. Forty-first Yearbook of the National Society for the Study of Education, Pt. II. Bloomington, Ill.: Public School Publishing Co., 1942. Pp. 377 411.

Horney, Karen. Self-Analysis. New York: W. W. Norton \& Company, Inc., 1942.

Hudgins, C. V. "Conditioning and Voluntary Control of the Pupillary Light Reflex," Journal of General Psychology, Vol. 8 (1933), pp. 3-51.

Hughes, Russell Meriwether (La Meri, pseud.). The Gesture Language of the Hindu Dance. New York: Columbia University Press, 1941.

Hull, Clark L. "Quantitative Aspects of the Evolution of Concepts," Psychological Review. Monograph Supplements, Vol. 28 (1920), pp. 1-86.

- "Knowledge and Purpose as Habit Mechanisms," Psychological Review, Vol. 37 (1930), pp. 511-25.

- "Goal Attraction and Directing Ideas Conceived as Habit Phenomena," Psychological Review, Vol. 38 (1931), pp. 487-506.

- Hypnosis and Suggestibility. New York: D. Appleton-Century Company, Inc., 1933.

- "The Concept of the Habit-Family Hierarchy and Maze Learning," Psychological Review, Vol. 41 (1934), pp. 33-54; 134-52.

- "The Mechanism of the Assembly of Behavior Segments in Novel Combinations Suitable for Problem Solution," Psychological Review, Vol. 42 (1935), pp. 219-45.

- "Mind, Mechanism, and Adaptive Behavior," Psychological Review, Vol. 44 (1937), pp. 1-32. 
- "The Problem of Stimulus Equivalence in Behavior Theory," Psychological Review, Vol. 46 (1939), pp. 9-30.

- "Modern Behaviorism and Psychoanalysis." Transactions of New York Academy of Sciences, Series II, Vol. 1 (1939), pp. 78-82.

- Principles of Behavior. New York: D. Appleton-Century Company, Inc., 1943.

Hunt, J. McV. (ed.). Personality and Behavior Disorders. 2 vols. New York: The Ronald Press Company, 1944.

Hunter, Walter S. "The Problem of Consciousness," Psychological Review, Vol. 31 (1924), pp. 1-31.

- "The Symbolic Process," Psychological Review, Vol. 31 (1924), pp. $478-97$.

pp. 153-70.

"The Subject's Report," Psychological Review, Vol. 32 (1925),

. "The Psychological Study of Behavior," Psychological Review, Vol. 39 (1932), pp. 1-24.

Husserl, Edmund. Logische Untersuchungen. Zweiter Band; I. Teil. Halle a S.: M. Niemeyer, 1901.

Ichheiser, Gustav. "The Image of the Other Man, a Study in Social Psychology," Sociometry, Vol. 3 (1940), pp. 277-91.

- "Misinterpretations of Personality in Everyday Life," Character and Personality, Vol. 12 (1943), pp. 145-60.

- "Structure and Dynamics of Interpersonal Relations," American Sociological Review, Vol. 8 (1943), pp. 302-5.

Isaacs, N. "The Logic of Language," Aristotelian Society Proceedings, Vol. 33 (1933), pp. 259-94.

Jackson, Reginald. "The Conventional Basis of Meaning," Aristotelian Society Proceedings, Vol. 33 (1933), pp. 203-36.

Jakobson, Roman. Kindersprache, Aphasie und allgemeine Lautgesetze. Uppsala: Sprakvetenskapliga Sällskapets i Uppsala Förhandlingar, 1940. 1942.

- Sound and Meaning. New York: Publications of the Linguistic Circle of New York. (Forthcoming.)

Janis, Irving L. "Meaning and the Study of Symbolic Behavior," Psychiatry, Vol. 6 (1943), pp. 425-39.

Jespersen, Otto. Language; Its Nature, Development and Origin. London: G. Allen \& Unwin, Ltd., 1922.

-The Philosophy of Grammar. New York: H. Holt and Company, 1924.

- The System of Grammar. London: G. Allen \& Unwin, Ltd., 1933.

- Analytic Syntax. London: G. Allen \& Unwin, Ltd., 1937. 
Johnson, Wendell. Language and Speech Hygiene: An Application of General Semantics. Chicago: Institute of General Semantics, 1939.

- "The Quantitative Study of Language Behavior," Psychological Bulletin, Vol. 38 (1941), p. 563. Abstract.

Johnson, W., Fairbanks, H., Mann, M., and Chotlos, J. Studies in Language Behavior. Psychological Monographs, Vol. 56 (1944), pp. 1-111.

Jones, Ernest. "The Theory of Symbolism," British Journal of Psychology, Vol. 9 (1917-19), pp. 181-229.

Jordan, Leo. "Essai de sociologie linguistique," Romanic Review, Vol. 20 (1929), pp. 305-25.

Juhos, B. von. "Some Modes of Speech of Empirical Science," Analysis, Vol. 3 (1936), pp. 65-74.

Jung, C. G. Psychology of the Unconscious. A Study of the Transformations and Symbolisms of the Libido. New York: Moffat, Yard and Company, 1916.

Kantor, J. R. "Language as Behavior and as Symbolism," Journal of Philosophy, Vol. 26 (1929), pp. 150-59.

- An Objective Psychology of Grammar. Bloomington, Indiana: Indiana University, 1936.

- Psychology and Logic. Bloomington, Indiana: The Principia Press, 1945.

Kaplan, Abraham. "Are Moral Judgments Assertions?” Philosophical Review, Vol. 51 (1942), pp. 280-303.

- The Language of Value: a Study in Pragmatics. Ph.D. Dissertation, University of California at Los Angeles, 1942.

- "Content Analysis and the Theory of Signs," Philosophy of Science, Vol. 10 (1943), pp. 230-47; with comments by Charles Morris, pp. 247-49.

Kaplan, Abraham, and Copilowish, Irving M. "Must There Be Propositions?" Mind, Vol. 48 (1939), pp. 478-84.

Kasanin, Jacob. Editor: Language and Thought in Schizophrenia. Berkeley: University of California Press, 1944.

Karlgren, Bernhard. Sound and Symbol in Chinese. London: Oxford University Press, 1923.

Kaufmann, Felix. "Truth and Logic," Philosophy and Phenomenological Research, Vol. 1 (1940), pp. 59-69.

- "Verification, Meaning, and Truth," Philosophy and Phenomenological Research, Vol. 4 (1943), pp. 267-83.

Methodology of the Social Sciences. London: Oxford University Press, 1944.

Kelsen, H. "The Pure Theory of Law and Analytical Jurisprudence," Harvard Law Review, Vol. 55 (1941), pp. 44-70. 
Kepes, György. Language of Vision. Chicago: Paul Theobald, 1944.

Kido, M. "On the Commutability and Limitation of Meaning in Language," Japanese Journal of Psychology, Vol. 9 (1934), pp. 1013-38. Original. English Abstract, pp. 71-72.

Kluckhohn, Clyde. "Myths and Rituals: A General Theory," Harvard Theological Review, Vol. 35 (1942), pp. 45-79.

- Navaho Witchcraft. Papers of the Peabody Museum of American Archaeology and Ethnology. Cambridge, Mass.: The Museum, 1944.

Kluckhohn, Clyde, and Kelly, W. H. "The Concept of Culture," The Science of Man in the World Crisis. Edited by Ralph Linton. New York: Columbia University Press, 1945.

Klüver, Heinrich. Behavior Mechanisms in Monkeys. Chicago: University of Chicago Press, 1933.

Koch, S. "The Logical Character of the Motivation Concept," Psychological Review, Vol. 48 (1941), pp. 15-38; 127-154.

Kokoszynska, Marja. "Über den absoluten Wahrheitsbegriff und einige andere semantische Begriffe," Erkenntnis, Vol. 6 (1936), pp. 143-65.

Korzybski, Alfred. Science and Sanity: An Introduction to Non-Aristotelian Systems and General Semantics. Lancaster, Pa.: The Science Press Printing Co., 1933. (2d ed., 1941.)

- "Neuro-Semantic and Neuro-Linguistic Mechanisms of Extensionalization," American Journal of Psychiatry, Vol. 93 (1936), pp. 29 38.

- "Science, Sanity, and Humanism," The Humanist, Vol. 2 (1942), pp. 140-45.

Krechel, Jakob. "Persönlichkeitstypus und Sprache," Archiv für die Gesamte Psychologie, Vol. 89 (1933), pp. 449-524.

Kroeber, A. L. "Sub-Human Culture Beginnings," Quarterly Review of Biology, Vol. 3 (1928), pp. 325-42.

Kris, Ernst. "Approaches to Art," Psychoanalysis Today. Edited by Sandor Lorand. New York: International University Press, 1944. Pp. 354-70.

- "Art and Regression," Transactions of the New York Academy of Sciences, Vol. 6 (1944), pp. 236-50.

Krout, Maurice H. "A Preliminary Note on Some Obscure Symbolic Muscular Responses of Diagnostic Value in the Study of Normal Subjects," American Journal of Psychiatry, Vol. 11 (1931), pp. 29-71.

- "Autistic Gestures: An Experimental Study in Symbolic Movement," Psychological Review. Monograph Supplements, Vol. 46 (1935), pp. 1-126. 
Kubie, Lawrence S. "Body Symbolization and the Development of Language," Psychoanalytic Quarterly, Vol. 3 (1934), pp. 430-44.

La Grasserie, Raoul de. Du Langage subjectif biologique ou émotionnel et sociologique ou révérentiel opposé au langage psychologique de la pensée. Paris: F. Leroux, 1907.

- Essai d'une sémantique intégrale. 2 vols. Paris: F. Leroux, 1908.

Landis, Carney. "Emotion: II. The Expression of Emotion." The Foundations of Experimental Psychology. Edited by Carl Murchison. Worcester, Mass.: Clark University Press, 1929. Pp. 488-523.

Langer, Susanne K. "A Logical Study of Verbs," Journal of Philosophy, Vol. 24 (1927), pp. 120-29.

- Philosophy in a New Key. Cambridge, Mass.: Harvard University Press, 1942.

Lanoe-Villène. Principes généraux de la symbolique des religions. Paris: Fischbacher, 1916.

Lashley, K. S. "Conditional Reactions in the Rat," The Journal of Psychology, Vol. 6 (1938), pp. 311-24.

Lasswell, Harold D. World Politics and Personal Insecurity. New York: McGraw-Hill Book Company, Inc., 1935.

- "A Provisional Classification of Symbol Data," Psychiatry, Vol. 1 (1938), pp. 197-204.

Lasswell, Harold D., and Associates. "The Politically Significant Content of the Press: Coding Procedures," Journalism Quarterly, Vol. 19, No. 1, March, 1942.

Lasswell, Harold D., Casey, Ralph D., and Smith, Bruce Lannes. Propaganda and Promotional Activities; an Annotated Bibliography. Minneapolis, Minn.: The University of Minnesota Press, 1935. (This bibliography is being continued by B. L. Smith in the Public Opinion Quarterly.)

Lasswell, H. D., Smith, B. L., Casey, R. D. Propaganda, Communication and Public Opinion: A Comprehensive Reference Guide. Princeton: Princeton University Press, 1946.

Lasswell, Harold D., and McDougal, Myres S. "Legal Education and Public Policy: Professional Training in the Public Interest," Yale Law Journal, Vol. 52 (1943), pp. 203-95.

Latif, Israil. "The Physiological Basis of Linguistic Development and of the Ontogeny of Meaning," Psychological Review, Vol. 41 (1934), pp. 5585.

Lee, Alfred McClung, and Lee, Elizabeth Briant. "The Fine Art of Propaganda," Institute for Propaganda Analysis, Inc. New York: Harcourt, Brace and Company, 1939. 
Lee, Irving J. Language Habits in Human Affairs: An Introduction to General Semantics. New York: Harper \& Brothers, 1941.

Leibniz's Metaphysics. Philosophical Classics. Translated by Dr. George R. Montgomery. The Religion of Science Library No. 50. Chicago: The Open Court Publishing Co., 1902.

Lepley, Ray. "The Verifiability of Facts and Values," Philosophy of Science, Vol. 5 (1938), pp. 310-20.

- "The Transposability of Facts and Values," The Journal of Philosophy, Vol. 36 (1939), pp. 290-99.

- "The Verifiability of Different Kinds of Facts and Values," Philosophy of Science, Vol. 7 (1940), pp. 464-75.

- Verifiability of Value. New York: Columbia University Press, 1944.

Levi, Albert William. "Language and Social Action," Ethics, Vol. 51 (1941), pp. 307-24.

Lewis, C. I. Mind and the World Order. New York: C. Scribner's Sons, 1929.

- "Experience and Meaning," Philosophical Review, Vol. 43 (1934), pp. 125-46.

- "The Modes of Meaning," Philosophy and Phenomenological Research, Vol. 4 (1943), pp. 236-49.

Lindner, Robert M. "An Experimental Study of Anticipation," American Journal of Psychology, Vol. 51 (1938), pp. 253-60.

Lindsay, Robert Bruce, and Margenau, Henry. Foundations of Physics. New York: J. Wiley \& Sons, Inc., 1936.

Link, George K. K. "The Rôle of Genetics in Etiological Pathology," The Quarterly Review of Biology, Vol. 8 (1932), pp. 127-71.

Littlefair, Duncan Elliot. "Logical Analysis of Concepts in Selected Systems of Theology." Ph.D. Dissertation, University of Chicago, 1940.

Loran, Erle. Cézanne's Compositions. Los Angeles: University of California Press, 1943.

Lorimer, Frank. The Growth of Reason. New York: Harcourt, Brace and Company, 1929.

Lundberg, George A. Foundations of Sociology. New York: The Macmillan Company, 1939.

Lundholm, Helge. "An Experimental Study of Functional Anesthesias as Induced by Suggestion in Hypnosis," The Journal of Abnormal and Social Psychology, Vol. 23 (1928), pp. 337-55.

Luria, A. R. The Nature of Human Conflicts. Translated by W. Gantt. New York: Liveright, Inc., 1932.

Macdonald, Margaret. “C. S. Peirce on Language," Psyche, Vol. 15 (1935), pp. 108-28. 
- "Language and Reference," Analysis, Vol. 4 (1936), pp. 33-41.

Mace, C. A. "Metaphysics and Emotive Language," Analysis, Vol. 2 (1934), pp. 6-10.

- "Representation and Expression," Analysis, Vol. 1 (1934), pp. 33-38.

MacKaye, James. The Logic of Language. Hanover, N. H.: Dartmouth College Publications, 1939.

Malinowski, Bronislaw. "Classificatory Particles in the Language of Kiriwina," Bulletin of the School of Oriental Studies, London Institution, Vol. 1, Pt. 4 (1920), pp. 33-78.

- Argonauts of the Western Pacific. New York: E. P. Dutton \& Co., 1922.

- "The Problem of Meaning in Primitive Languages," in C. K. Ogden's and I. A. Richards' The Meaning of Meaning. New York: Harcourt, Brace and Company, 1923.

-. "Magic, Science, and Religion," in Essays collected by J. Needham under the title Science, Religion, and Reality. New York: The Macmillan Company, 1926.

- "The Role of Myth in Life," Psyche, Vol. 6 (1926), pp. 29-39.

- Myth in Primitive Psychology. New York: W. W. Norton \& Company, Inc., 1926.

- "Culture," Encyclopedia of the Social Sciences, Vol. 4 (1931), pp. 621-45.

- Coral Gardens and Their Magic. London: G. Allen \& Unwin, Ltd., 1935.

- "The Group and the Individual in Functional Analysis," American Journal of Sociology, Vol. 44 (1939), pp. 938-64.

Mannheim, Karl. Man and Society in an Age of Reconstruction. Translated by Edward Shils. New York: Harcourt, Brace and Company, 1940.

Mannoury, Gerrit. "Die signifischen Grundlagen der Mathematik," Erkenntnis, Vol. 4 (1934), pp. 288-309; 317-45.

- "Signifische Analyse der Willenssprache," Erkenntnis, Vol. 7 (1938), pp. 180-88.

Markey, John F. The Symbolic Process and Its Integration in Children. New York: Harcourt, Brace and Company, 1928.

Masserman, Jules H. "Psychobiologic Dynamisms in Behavior," Psychiatry: Journal of the Biology and Pathology of Interpersonal Relations, Vol. 5 (1942), pp. 341-47.

1943. 
- "Language Behavior and Dynamic Psychiatry," International Journal of Psychoanalysis, Vol. 25 (1944), pp. 1-8.

- Principles of Dynamic Psychiatry. Philadelphia: W. B. Saunders \& Co., 1945.

Masserman, Jules H., and Balken, Eva R. "The Psychoanalytic and Psychiatric Significance of Phantasy," Psychoanalytic Review, Vol. 26 (1939), pp. 343-79; 535-49.

- "Use of Fantasy Tests in Differential Psychiatric Diagnosis," Archives of Neurology and Psychiatry, Vol. 43 (1940), pp. 1249-55.

Masserman, Jules, H., and Rubinfine, D. L. " 'Counting' Behavior in Cats," Journal of General Psychology, Vol. 30 (1944), pp. 87-88.

Mathesius, Vilém. "On Some Problems of the Systematic Analysis of Grammar," Travaux du cercle linguistique de Prague, Vol. 6 (1936), pp. 95-107.

McConnel, Ursula Hope. “Symbolism as a Mental Process," Psyche, Vol. 12 (1931), pp. 37-51.

- "The Symbol in Legend," Psyche, Vol. 13 (1933), pp. 94-137.

McDougall, K. D., and McDougall, W. "Insight and Foresight in Various Animals," Journal of Comparative Psychology, Vol. 11 (1931), pp. 23773.

McKeon, Richard P. "Rhetoric in the Middle Ages," Speculum, Vol. 17 (1942), pp. 1-32.

Mead, George H. "The Relations of Psychology and Philology," The Psychological Bulletin, Vol. 1 (1904), pp. 375-91.

- "Concerning Animal Perception," Psychological Review, Vol. 14 (1907), pp. 383-90.

- "Social Consciousness and the Consciousness of Meaning," The Psychological Bulletin, Vol. 7 (1910), pp. 397-405.

- "The Mechanism of Social Consciousness," Journal of Philosophy, Vol. 9 (1912), pp. 401-06.

- "A Behavioristic Account of the Significant Symbol," Journal of Philosophy, Vol. 19 (1922), pp. 157-63.

- "The Genesis of the Self and Social Control," International Journal of Ethics, Vol. 35 (1925), pp. 251-77.

- "Cooley's Contribution to American Social Thought," The American Journal of Sociology, Vol. 35 (1930), pp. 693-706.

- Mind, Self and Society. Chicago: University of Chicago Press, 1934.

Press, 1938.

Mead, Margaret. Sex and Temperament in Three Primitive Societies. New York: W. Morrow \& Company, 1935. 
Menger, Karl. "A Logic of the Doubtful. On Optative and Imperative Logic," Reports of a Mathematical Colloquium, Second Series, Issue 1. Notre Dame, Indiana: University Press, 1939. Pp. 53-64.

Miller, David L. "The Calendar Theory of Freedom," Journal of Philosophy, Vol. 41 (1944), pp. 320-28.

Miller, Neal E. "A Reply to 'Sign-Gestalt or Conditioned Retlex," Psychological Review, Vol. 42 (1935), pp. 280-92.

Miller, Neal E., and Dollard, John. Social Learning and Imitation. New Haven: Yale University Press, 1941.

Mills, C. Wright. "Language, Logic and Culture," American Sociological Review, Vol. 4 (1939), pp. 670-80.

Mises, Richard von. Kleines Lehrbuch des Positivismus. Einführung in die empiristiche Wissenschaftsauffassung. The Hague, Holland: W. P. Van Stockum \& Zoon, 1939.

- Probability, Statistics and Truth. New York: The Macmillan Company, 1939.

Moore, Willis. "Structure in Sentence and Fact," Philosophy of Science, Vol. 5 (1938), pp. 81-88.

More, Adelyne. "Opposition-Logical, Psychological, and Orthological," Psyche, Vol. 10 (1930), pp. 29-52.

Morgan, C. Lloyd. "Meaning and Significance," The Journal of Experimental Pedagogy, Vol. 3 (1915), pp. 1-8.

Morris, Charles. "The Concept of the Symbol, I," Journal of Philosophy, Vol. 24 (1927), pp. 253-62.

- "The Concept of the Symbol, II," Journal of Philosophy, Vol. 24 (1927), pp. 281-91.

1932.

- "The Relation of Formal and Empirical Sciences within Scientific Empiricism," Erkenntnis, Vol. 5 (1935), pp. 6-14.

-. Logical Positivism, Pragmatism, and Scientific Empiricism. Actualités scientifiques et industrielles, No. 449. Paris: Herman et Cie, 1937.

- Foundations of the Theory of Signs. International Encyclopedia of Unified Science, Vol. 1, No. 2. Chicago: University of Chicago Press, 1938.

-1. "Science, Art and Technology," Kenyon Review, Vol. 1 (1939), pp. $409-23$.

- "Esthetics and the Theory of Signs," Journal of Unified Science (Erkenntnis), Vol. 8 (1939), pp. 131-50.

- "General Education and the Unity of Science Movement," John 
Dewey and the Promise of America, Progressive Education Booklet, No. 14 (1939), pp. 26-40.

- "The Mechanism of Freedom," Freedom, Its Meaning. Edited by Ruth N. Anshen. New York: Harcourt, Brace and Company, 1940. - "The Search for a Life of Significance. The Work of Raymond Jonson, American Painter," Tomorrow, Vol. 1 (1941), pp. 16-21.

- "Empiricism, Religion, and Democracy," Second Conference on Science, Philosophy, and Religion, 1942.

- - Paths of Life: Preface to a World Religion. New York: Harper and Brothers, 1942.

Mowrer, O. H. "A Stimulus-Response Analysis of Anxiety and Its Role as a Reinforcing Agent," Psychological Review, Vol. 46 (1939), pp. 553 65 .

-. "Preparatory Set (Expectancy)-Some Methods of Measurement," Psychological Review. Monograph Supplements, Vol. 52 (1940). Pp. 43.

Muller, Herbert J. Science and Criticism. New Haven: Yale University Press, 1943.

Nagel, Ernest. "Verifiability, Truth, and Verification," Journal of Philosophy, Vol. 31 (1934), pp. 141-48.

- "Some Theses in the Philosophy of Logic," Philosophy of Science, Vol. 5 (1938), pp. 46-51.

-. "Truth and Knowledge of the Truth," Philosophy and Phenomenological Research, Vol. 5 (1944), pp. 50-68.

- Principles of the Theory of Probability. International Encyclopedia of Unified Science, Vol. 1, No. 6. Chicago: University of Chicago Press, 1939.

- "Logic without Ontology," Naturalism and the Human Spirit. Edited by Yervant H. Krikorian. New York: Columbia University Press, 1944.

Neurath, Otto. Einheitswissenschaft und Psychologie. Wien: Gerold \& Co., 1933.

- International Picture Language, the First Rules of Isotype. London: K. Paul, Trench, Trubner \& Co., Ltd., 1936.

Ltd., 1936.

- Modern Man in the Making. New York: A. A. Knopf, 1939. - "Universal Jargon and Terminology," Aristotelian Society Proceedings, Vol. 44 (1940-41), pp. 127-48.

- Foundations of the Social Sciences. International Encyclopedia of Unified Science, Vol. 2, No. 1. Chicago: University of Chicago Press, 1944. 
Newman, S. S. "Further Experiments in Phonetic Symbolism," American Journal of Psychology, Vol. 45 (1933), pp. 53-75.

- "Personal Symbolism in Language Patterns," Psychiatry, Vol. 2 (1939), pp. 177-84.

Niceforo, A. "La personalité et le langage," Revue de l'institut de sociologie, Vol. 2 (1929), pp. 317-60; Vol. 3 (1929), pp. 571-610.

Nicholls, Frederick. The Language of Music, or, Musical Expression and Characterization. London: K. Paul, Trench, Trubner and Co., Ltd., 1924.

Nissen, H. W., and Crawford, M. P. "A Preliminary Study of FoodSharing Behavior in Young Chimpanzees," Journal of Comparative Psychology, Vol. 22 (1936), pp. 383-419.

Ogden, C. K. "Bentham's Philosophy of As-If," Psyche, Vol. 9 (1928), pp. 4-14.

- Opposition, a Linguistic and Psychological Analysis. Psyche Miniatures, General Series, No. 41. London: K. Paul, Trench, Trubner \& Co., Ltd., 1932.

3-43.

"Bentham's Theory of Language," Psyche, Vol. 12 (1932), pp.

. Bentham's Theory of Fictions. International Library of Psychology, Philosophy and Scientific Method. New York: Harcourt, Brace and Company, 1932.

- "The Magic of Words," Psyche, Vol. 14 (1934), pp. 9-87.

Ogden, C. K., and Richards, I. A. The Meaning of Meaning. London: K. Paul, Trench, Trubner \& Co., Ltd., 1923. 5th ed. revised. New York: Harcourt, Brace and Company, 1938.

Oldfield, R. C. "Towards a Functional Theory of Word-Meaning," Psyche, Vol. 15 (1935), pp. 129-37.

Oppenheim, Félix. "Outline of a Logical Analysis of Law." Ph.D. Dissertation, Princeton University, 1943. Also in Philosophy of Science, Vol. 11 (1944), pp. 142-60.

Osgood, Charles E. Meaningful Similarity and Interference in Learning. Ph.D. Dissertation. New Haven: Yale University Press, 1944.

Paget, Richard. "The Origin of Language," Psyche, Vol. 8 (1927), pp. 35 39.

Palmer, H. E. “Word Values," Psyche, Vol. 9 (1928), pp. 13-25.

Park, Robert E. "Reflections on Communication and Culture," The American Journal of Sociology, Vol. 44 (1938), pp. 187-205.

- "Symbiosis and Socialization: A Frame of Reference for the Study of Society," The American Journal of Sociology, Vol. 45 (1939), pp. 1-25. 
Parsons, Howard. Myth and Religious Knowledge. Ph.D. Dissertation, University of Chicago, 1946.

Parsons, Talcott. "The Role of Ideas in Social Action," American Sociological Review, Vol. 3 (1938), pp. 652-64.

Pavlov, I. P. Conditioned Reflexes. Translated by G. V. Anrep. London: Oxford University Press, 1927.

Peirce, Charles Sanders. Collected Papers. Cambridge, Mass.: Harvard University Press, Vol. 1, 1931; Vol. 2, 1932; Vol. 4, 1933; Vol. 5, 1934; Vol. 6, 1935.

Penttilä, Aarni, and Saarnio, Uuno. "Einige grundlegende Tatsachen der Worttheorie nebst Bemerkungen über die sogenannten unvollständigen Symbole," Erkenntnis, Vol. 4 (1934), pp. 28-45; $139-59$.

Pepper, Stephen L. World Hypotheses. Los Angeles: University of California Press, 1942.

Perry, Ralph Barton. “A Behavioristic View of Purpose," Journal of Philosophy, Vol. 18 (1921), pp. 85-105.

- "The Independent Variability of Purpose and Belief," Journal of Philosophy, Vol. 18 (1921), pp. 169-80.

Piaget, Jean. The Language and Thought of the Child. New York: Harcourt, Brace and Company, 1926.

- Judgment and Reasoning in the Child. New York: Harcourt, Brace and Company, 1928.

- The Moral Judgment of the Child. London: K. Paul, Trench, Trubner \& Co., Ltd., 1932.

Pollock, Thomas Clark. "A Critique of I. A. Richards' Theory of Language and Literature," $A$ Theory of Meaning Analyzed, General Semantics Monographs, No. 3. Chicago: Institute of General Semantics, 1942.

- The Nature of Literature, Its Relation to Science, Language and Human Experience. Princeton: Princeton University Press, 1942.

Popper, Karl R. "What Is Dialectic?" Mind, Vol. 49 (1940), pp. 403-26.

Pos, H. J. "The Philosophical Significance of Comparative Semantics," Philosophy and History. Oxford: The Clarendon Press, 1936. Pp. 26576.

Prantl, Carl von. Geschichte der Logik im Abendlande. Leipzig: S. Hirzel, 1855-70.

Quine, Willard V. "Designation and Existence," Journal of Philosophy, Vol. 36 (1939), pp. 701-709.

- "Notes on Existence and Necessity," Journal of Philosophy, Vol. 40 (1943), pp. 113-27.

Rand, Rose. "Logik der Forderungssätze," Internationale Zeitschrift für Theorie des Rechts, Vol. 1 (1939), pp. 308-22. 
Rank, Otto. Psychoanalytische Beiträge zur Mythenforschung aus den Jahren 1912 bis 1914. Leipzig: Internationaler psychoanalytischer Verlag, 1922.

Ransom, John Crowe. The New Criticism. Norfolk, Conn.: New Directions, 1941.

Reach, Karel. “What Is a Language?" Analysis, Vol. 6 (1939), pp. 49-62.

Redlick, F. C. "The Patient's Language. An Investigation into the Use of Medical Terms," Yale Journal of Biology and Medicine, Vol. 17 (1945), pp. 427-53.

Reichenbach, Hans. Experience and Prediction. Chicago: University of Chicago Press, 1938.

- Philosophic Foundations of Quantum Mechanics. Berkeley: University of California Press, 1944.

Rhine, Raymond. "Explicit Denotation in Language: A Psychological Contribution to Methods in the Social Sciences," The Journal of Social Psychology, Vol. 18 (1943), pp. 331-63.

Rice, Philip Blair. "Toward a Syntax of Valuation," Journal of Philosophy, Vol. 41 (1944), pp. 309-20.

Richards, I. A. Principles of Literary Criticism. New York: Harcourt, Brace \& Company, 1925. 4th ed., 1930.

- Science and Poetry. New York: W. W. Norton \& Company, 1926.

-. Practical Criticism. 3d ed. London: K. Paul, Trench, Trubner \& Co., Ltd., 1935. (First published in 1929.)

- Mencius on the Mind. Experiments in Multiple Definition. London: K. Paul, Trench, Trubner \& Co., Ltd., 1932.

- Basic Rules of Reason. Psyche Miniatures, General Series, No. 62. London: K. Paul, Trench, Trubner \& Co., Ltd., 1933.

-. "Preface to a Dictionary," Psyche, Vol. 13 (1933), pp. 10-24.

- Coleridge on Imagination. New York: Harcourt, Brace and Company, 1935.

-. "Logical Machinery," Psyche, Vol. 16 (1936), pp. 76-99.

- The Philosophy of Rhetoric. London: Oxford University Press, 1936.

- "Basic English in the Study of Interpretation," Psyche, Vol. 17 (1937), pp. 35-47.

- Interpretation and Teaching. New York: Harcourt, Brace and Company, 1938.

- How to Read a Page. New York: W. W. Norton \& Company, Inc., 1942.

Rickert, Edith. New Methods for the Study of Literature. Chicago: University of Chicago Press, 1927. 
Ricklin, Franz. Wishfulfillment and Symbolism in Fairy Tales. Nervous and Mental Disease Monographs, No. 21. New York, 1915.

Riesen, Austin H. Delayed Reward in Discrimination Learning by Chimpanzees. Baltimore, Md.: The Johns Hopkins Press, 1940. Also Comparative Psychology Monographs, Vol. 15 (1940).

Rieser, Max. "Analysis of the Poetic Simile," Journal of Philosophy, Vol. 37 (1940), pp. 209-17.

Riezler, Kurt. "What Is Public Opinion?" Social Research, Vol. 11 (1944), pp. 397-427.

Ritchie, Benbow F., and Kaplan, Abraham. "A Framework for an Empirical Ethics," Philosophy of Science, Vol. 7 (1940), pp. 476-91.

Robach, A. A. A Dictionary of International Slurs. Cambridge, Mass.: Sci-art Publishers, 1944.

Rossiter, A. P. "Poetry as Gagagram: An Inquiry into Meaning," Psyche, Vol. 15 (1935), pp. 174-87.

Rowland, Eleanor H. "The Psychological Experiences Connected with the Different Parts of Speech," Psychological Review, Monograph Supplements, Vol. 8 (1907), pp. 1-42.

Russell, Bertrand. "The Meaning of Meaning," Mind, Vol. 29 (1920), pp. 398-404.

- "On Verification," Aristotelian Society Proceedings, Vol. 38 (193738), pp. 1-20.

- An Inquiry into Meaning and Truth. New York: W. W. Norton \& Company, Inc., 1940.

Ryan, T. A. "Mathematical Objects and Symbolizing," American Journal of Psychology, Vol. 51 (1938), pp. 283-303.

Saarnio, Uuno. "Zur heterologischen Paradoxie," Theoria, Vol. 1 (1937), pp. 38-56.

Sanford, Fillmore H. Individual Differences in the Mode of Verbal Expression. Ph.D. Dissertation. Widener Library, Harvard University, 1941.

- "Speech and Personality," Psychological Bulletin, Vol. 39 (1942), pp. 811-45. Also in Character and Personality, Vol. 10 (1942), pp. 169. 98.

Sapir, Edward. "The History and Varieties of Human Speech," Popular Science Monthly, Vol. 79 (1911), pp. 45-67.

-. "Language and Environment," American Anthropologist, Vol. 14 (1912), pp. 226-42. $37-51$.

- "Language as a Form of Human Behavior," The English Journal, Vol. 16 (1927), pp. 421-33. 
" "Speech as a Personality Trait," The American Journal of Sociology, Vol. 32 (1927), pp. 892-905.

"The Status of Linguistics as a Science," Language, Vol. 5 (1929), pp. 207-14.

- "A Study in Phonetic Symbolism," Journal of Experimental Psychology, Vol. 12 (1929), pp. 225-39.

—. "Totality," Language Monographs, No. 6, 1930.

-. "Communication," Encyclopedia of the Social Sciences, Vol. 4 (1931), pp. 78-80.

- "Language," Encyclopedia of the Social Sciences, Vol. 9 (1933), pp. 155-68.

- "Symbolism," Encyclopedia of the Social Sciences, Vol. 14 (1934), pp. 492-95.

Schächter, Josef. Prolegomena zu einer kritischen Grammatik. Wien: Julius Springer, 1935.

Schilder, Paul. "The Psychology of Schizophrenia," The Psychoanalytic Review, Vol. 26 (1939), pp. 380-98.

Schiller, F. C. S. Logic for Use. New York: Harcourt, Brace and Company, 1930.

Schiller, F. C. S., Russell, B., and Joachim, H. H. "The Meaning of Meaning, a Symposium," Mind, Vol. 29 (1920), pp. 385-97.

Schlick, Moritz. "Meaning and Verification," Philosophical Review, Vol. 45 (1936), pp. 339-69.

- Gesammelte Aufsätze. Vienna: Gerold \& Co., 1938.

Senior, James K. "On Certain Relations between Chemistry and Geometry," Journal of Chemical Education, Vol. 15 (1938), pp. 464-70.

Seth, George, "Psychological Aspects of Contemporary Poetry," British Journal of Psychology, Vol. 27 (1936), pp. 425-35.

Sheffield, Alfred D. Grammar and Thinking: a Study of the Working Conceptions in Syntax. New York: G. P. Putnam's Sons, 1912.

Sheldon, W. H. The Varieties of Temperament. New York: Harper \& Brothers, 1942.

Sidgwick, Alfred. "Interpretation of Words," Mind, Vol. 37 (1928), pp. 149-72.

Silberer, Herbert. "Zur Symbolbildung," Jahrbuch für psychoanalytische und psychopathologische Forschungen, Vol. 4 (1912), pp. 607-83.

- Problems of Mysticism and Its Symbolism. Translated by S. E. Jelliffe. New York: Moffat, Yard and Company, 1917.

Skinner, B. F. "The Concept of the Reflex in the Description of Behavior," Journal of General Psychology, Vol. 5 (1931), pp. 427-58.

-. "The Verbal Summator and a Method for the Study of Latent Speech," Journal of Psychology, Vol. 2 (1936), pp. 71-107. 
- The Behavior of Organisms. New York: D. Appleton-Century Company, Inc., 1938.

- "The Operational Analysis of Psychological Terms," Psychological Review, Vol. 52 (1945), pp. 270-77.

Smart, Harold R. "The Unit of Discourse," The Philosophical Review, Vol. 50 (1941), pp. 268-88.

- "The Classification of the Elements of Discourse," The Philosophical Review, Vol. 52 (1943), pp. 233-51.

Smith, W. S. "Communication-the Function of Symbols in Society," Society under Analysis. Edited by Elmer Pendell. Lancaster, Pa.: The Jaques Cattell Press, 1942. Pp. 290-323.

Sondel, Bess. Speak Up! A New Approach to Communication. Chicago: University of Chicago Bookstore, 1944.

- Are You Telling Them? New York: Prentice-Hall, Inc. (Forthcoming.)

Spence, Kenneth W. "Experimental Studies of Learning and the Higher Mental Processes in Infra-Human Primates," Psychological Bulletin, Vol. 34 (1937), pp. 806-50.

Spier, Leslie, Hallowell, A. Irving, and Newman, Stanley S. (editors). Language, Culture and Personality. Menasha, Wis.: Sapir Memorial Publication Fund, 1941.

Stace, W. T. "Positivism," Mind, Vol. 53 (1944), pp. 215-37.

Stallknecht, Newton. "The Place of Verification in Ethical Theory," Journal of Philosophy, Vol. 34 (1937), pp. 150-56.

Stebbing, L. S. "Sounds, Shapes, and Words," Science, History, and Theology, Aristotelian Society Supplementary Series, Vol. 14 (1935), pp. 1-21. Stern, Gustaf. Meaning and Change of Meaning; with Special Reference to the English Language. Göteborg: Elanders boktryckeri aktiebolag, 1931.

Stevens, S. S. "Psychology and the Science of Science," Psychological Bulletin, Vol. 36 (1939), pp. 221-63.

Stevenson, Charles Leslie. "The Emotive Meaning of Ethical Terms," Mind, Vol. 46 (1937), pp. 14-31.

- "Ethical Judgments and Avoidability," Mind, Vol. 47 (1938), pp. $45-57$.

—_. "Persuasive Definitions," Mind, Vol. 47 (1938), pp. 331-50.

-. Ethics and Language. New Haven: Yale University Press, 1944. Sullivan, Harry Stack. "Peculiarity of Thought in Schizophrenia," American Journal of Psychiatry, Vol. 5 (1925-26), pp. 21-86.

- "The Importance of a Study of Symbols in Psychiatry," Psyche, Vol. 7 (1926), pp. 81-93. 
Symons, N. J. "A Note on the Formation of Symbols," International Journal of Psychoanalysis, Vol. 6 (1925), pp. 440-43.

Tarski, Alfred. "Fundamentale Begriffe der Methodologie de deduktiven Wissenschaften, I," Monatshefte für Mathematik und Physik, Vol. 37, H2 (1930), pp. 361-404.

- "Der Wahrheitsbegriff in den formalisierten Sprachen," Studia Philosophica, Vol. 1 (1935), pp. 261-405.

- "Grundlegung der wissenschaftlichen Semantik," Actualités scientifiques et industrielles, No. 390. Actes du congrès international de philosophie scientifique. Paris: Hermann \& Cie, 1936. Pp. 1-8.

- "The Semantic Conception of Truth," Philosophy and Phenomenological Research, Vol. 4 (1944), pp. 341-76.

Tate, Allen. "Literature as Knowledge: Comment and Comparison," Southern Review, Vol. 6 (1940-1941), pp. 629-57.

-. Reason in Madness. New York: G. P. Putnam's Sons, 1941.

Thomas, George F. "Myth and Symbol in Religion," Journal of Bible and Religion, Vol. 7 (1939), pp. 163-71.

Tillich, Paul. "The Religious Symbol," The Journal of Liberal Religion, Vol. 2 (1940), pp. 13-36.

Tinbergen, N. "An Objectivistic Study of the Innate Behavior of Animals," Biblioteca Biotheoretica, Series D, Vol. 1, pt. 2, 1942, pp. 39-98.

Tolman, Edward Chace. "A Behavioristic Theory of Ideas," Psychological Review, Vol. 33 (1926), pp. 352-69.

- Purposive Behavior in Animals and Men. New York: The Century Co., 1932.

- "Gestalt and Sign-Gestalt," Psychological Review, Vol. 40 (1933), pp. 391-411.

- "Psychology versus Immediate Experience," Philosophy of Science, Vol. 2 (1935), pp. 356-80.

-. "Connectionism; Wants, Interests, and Attitudes," Character and Personality, Vol. 4 (1936), pp. 245-53.

- "The Acquisition of String-Pulling by Rats-Conditioned Response or Sign-Gestalt?" Psychological Review, Vol. 44 (1937), pp. 195-211.

- "Physiology, Psychology and Sociology," Psychological Review, Vol. 45 (1938), pp. 228-41.

- "Motivation, Learning, and Adjustment," Proceedings of the American Philosophical Society, Vol. 84, No. 4 (1941), pp. 543-63.

Tolman, E. C., and Brunswik, Egon. "The Organism and the Causal Texture of the Environment," Psychological Review, Vol. 42 (1935), pp. 43-77. 
Tolman, E. C., and Krechevsky, I. "Means-End-Readiness and Hypothesis -A Contribution to Comparative Psychology," Psychological Review, Vol. 40 (1933), pp. 60-70.

Travis, Lee Edward. Speech Pathology. New York: D. Appleton and Company, 1931.

Tsuru, S., and Fries, H. S. "A Problem in Meaning," Journal of General Psychology, Vol. 8 (1933), pp. 281-84.

Urban, Wilbur M. "Value Propositions and Verifiability," Journal of Philosophy, Vol. 34 (1937), pp. 589-602.

- "Symbolism in Science and Philosophy," Philosophy of Science, Vol. 5 (1938), pp. 276-99.

- Language and Reality; the Philosophy and the Principles of Symbolism. New York: The Macmillan Company, 1939.

Ushenko, A. "On the Meaning of a Proposition," Journal of Philosophy, Vol. 28 (1931), pp. 715-16.

Vaihinger, Hans. 'The Philosophy of 'As If.' Translated by C. K. Ogden. London: K. Paul, Trench, Trubner \& Co., Ltd., 1924. (First published in German in 1911.)

Velten, H. V. "The Science of Language and the Language of Science," Publications of the Modern Language Association, Vol. 48 (1933), pp. 608-22.

Venable, Vernon. "Poetic Reason in Thomas Mann," Virginia Quarterly Review, Vol. 14 (1938), pp. 61-76.

Verlaine, L. "L'instinct et l'intelligence chez les hymenoptères," Annales de la société royale de Belgique, Vol. 58 (1927), pp. 59-88.

Vigotsky, S. "Thought in Schizophrenia," Archives of Neurology and Psychiatry, Vol. 31 (1934), pp. 1063-77.

Voloshinov, V. Marksizm i filosofiia iazyka. Leningrad: Gosudarstvennoe izdatel'stvo R.S.F.S.R., 1930.

Waismann, Friedrich. Einführung in das mathematische Denken; die Begriffsbildung der modernen Mathematik. Wien: Gerold \& Co., 1936.

- "Von der Natur eines philosophischen Problems," Synthese, Vol. 1 (Juli, 1939), pp. 340-50; Vol. 2 (August, 1939), pp. 395-406.

Wallerand, G. Les Oeuvres de Siger de Courtrai. Louvain: Institut supérieur de philosophie de l'université, 1913.

Wallis-Walfisz, Mielczyslaw. "L’Art au point de vue sémantique: une méthode récente d'esthétique," Congrès international d'esthétique et de science de l'art. 2d Congress. Vol. 1 (1937), pp. 17-21.

Walpole, Hugh. Semantics. New York: W. W. Norton \& Company, Inc., 1941.

Walsh, Dorothy. "The Poetic Use of Language," Journal of Philosophy, Vol. 35 (1938), pp. 73-81. 
Waples, D. (ed.). Print, Radio, and Film in a Democracy. Chicago: University of Chicago Press, 1942.

Warner, W. Lloyd. "The Society, the Individual, and His Mental Disorders," American Iournal of Psychiatry, Vol. 94 (1937), pp. 275-84.

- A Black Civilization. New York: Harper and Brothers, 1937.

Watson, John B. Behavior; an Introduction to Comparative Psychology. New York: H. Holt and Company, 1914.

- "Behavior and the Concept of Mental Disease," Journal of Philosophy, Vol. 13 (1916), pp. 589-97.

- "The Place of the Conditioned-Reflex in Psychology," Psychological Review, Vol. 23 (1916), pp. 89-116.

- Psychology from the Standpoint of a Behaviorist. Philadelphia: J. B. Lippincott Company, 1919.

- Behaviorism. New York: The People's Institute Publishing Company, Inc., 1924.

Weber, Louis. "De quelques caractères de la pensée symbolique," Revue de métaphysique et de morale, Vol. 36 (1929), pp. 156-76; 343-59.

Weinhandl, Ferdinand. Über das aufschliessende Symbol. Berlin: Junker und Dünnhaupt, 1929.

Weiss, Albert P. "Linguistics and Psychology," Language, Vol. 1 (1925), pp. 52-57.

Weitz, Morris. "Does Art Tell the Truth?" Philosophy and Phenomenological Research, Vol. 3 (1943), pp. 338-48.

Welby, Lady V. Significs and Language. London: Macmillan and Co., 1911.

Wheeler, R. H. "The Development of Meaning," American Journal of Psychology, Vol. 33 (1922), pp. 223-33.

Wheelwright, Philip. "On the Semantics of Poetry," Kenyon Review, Vol. 2 (1940), pp. 263-83.

Whetnall, E. M. "Symbol Situations," Aristotelian Society Proceedings, Vol. 29 (1929), pp. 191-226.

White, W. A. "The Language of Schizophrenia," Archives of Neurology and Psychiatry, Vol. 16 (1926), pp. 395-413.

Whitehead, Alfred North. Symbolism, Its Meaning and Effect. New York: The Macmillan Company, 1927.

Wick, Warner Arnis. Metaphysics and the New Logic. Chicago: University of Chicago Press, 1942.

Wieman, Henry Nelson. "On Using Christian Words," Journal of Religion, Vol. 20 (1940), pp. 257-69.

_- "Theology and the Philosophy of Religion: A Comparison and a Contrast," The Jourral of Liberal Religion, Vol. 2 (1941), pp. 163-75. 
Williams, Donald C. "Tokens, Types, Words and Terms," Journal of Philosophy, Vol. 33 (1936), pp. $701-07$.

- "The Realistic Interpretation of Scientific Sentences," Erkenntnis, Vol. 7 (1938), pp. 169-78.

Williams, Katherine Adams. "The Conditioned Reflex and the Sign Function in Learning," Psychological Review, Vol. 36 (1929), pp. 481-97.

- "The Reward Value of a Conditioned Stimulus," University of California Publications in Psychology, Vol. 4 (1929), pp. 31-55.

Willoughby, Raymond R. "The Functions of Conversation," lournal of Social Psychology, Vol. 3 (1932), pp. 146-60.

- "Magic and Cognate Phenomena: an Hypothesis," $A$ Handbook of Social Psychology. Worcester, Mass.: Clark University Press, 1935. Pp. 461-519.

Wisdom, John. Interpretation and Analysis. Psyche Miniatures, General Series No. 35. London: K. Paul, Trench, Trubner \& Co., Ltd., 1931.

-Metaphysics and Verification," Mind, Vol. 47 (1938), pp. $452-98$. Wittgenstein, Ludwig. Tractatus Logico-Philosophicus. New York: Harcourt, Brace and Company, 1922.

Wodehouse, Helen. "Language and Moral Philosophy," Mind, Vol. 47 (1938), pp. 200-13.

- 'On Richards' Interpretation in Teaching," Mind, Vol. 48 (1939), pp. 227-36.

Wölfflin, Heinrich. Kunstgeschichtliche Grundbegriffe. München: H. Bruckmann, 1923.

Woods, W. L. "Language Study in Schizophrenia," Journal of Nervous and Mental Diseases, Vol. 87 (1938), pp. 290-316.

Yerkes, Robert M. Chimpanzees, a Laboratory Colony. New Haven: Yale University Press, 1943.

Yerkes, Robert M., and Nissen, Henry W. "Pre-linguistic Sign Behavior in Chimpanzee," Science, Vol. 89 (1939), pp. 585-87.

Young, Kimball. "Language, Thought, and Social Reality," Social Attitudes. Edited by K. Young. New York: H. Holt and Company, 1931. Pp. 100-35.

Zener, Karl. "The Significance of Behavior Accompanying Conditioned Salivary Secretion for Theories of the Conditioned Response," American Journal of Psychology, Vol. 50 (1937), pp. 384-403.

Zipf, George K. The Psycho-biology of Language: an Introduction to Dynamic Philology. Boston: Houghton Miflin Company, 1935. 


\section{Collections of Articles}

Acta linguistica, revue internationale de linguistique structurale, Vol. 1 (1939).

Etc., A Review of General Semantics, Vol. 1, 1942.

General Semantics: Papers from the First American Congress for General

Semantics 1935. Distributed by Arrow Editions, New York, 1938.

Journal de psychologie. Vol. 18, 1921. Vol. 30, 1933.

Mind. Vol. 46, 1937. Vol. 47, 1938.

Papers from the Second American Congress on General Semantics 1941.

Chicago: Institute of General Semantics, 1943.

Psychological Bulletin. Vol. 25, No. 6, 1929. Pp. 240-338.

Psychological Review. Vol. 31, 1924. Vol. 32, 1925.

Travaux du cercle linguistique de Prague. Vols. 1-8. 1929-1939.

"Die Wissenschaftliche Sprache," Vierter Internationaler Kongress für Einheit der Wissenschaft. Erkenntnis, Vol. 7 (1938), pp. 135-246. 


\section{GLOSSARY}

THIS glossary includes the main terms of the present system of semiotic. A few terms from general behavior theory are included, but since they are not defined in terms of signs, they really operate as undefined terms in this system. This glossary is not an index; it does not include many of the topics treated in the text nor the discussion of terms as employed by other semioticians. Its sole purpose is to help the reader keep at hand the main terminology adopted in this work. The terms that are starred are the most important and provide the basis for the definitions of the other terms.

A

ADEQUacy

A sign is adequate to the degree to which it achieves the purpose for which it is used.

Ambiguous SIGN A sign-vehicle that is not unambiguous.

ANALYTIC INTERPRETANTS Interpretants so related that if one is prevented from issuing in overt behavior, that fact alone supplies the conditions for the other to issue in overt behavior.

Appraisal ................... An appraisive ascriptor considered as produced by some interpreter.

*APpraisor

A sign that signifies something as having a preferential status for behavior. A significatum of such a sign is called a valuatum. Positive and negative appraisors are distinguished; also, utilitators and consummators, depending upon whether what is signified is a means-object or a goalobject.

"Ascriptor

A sign complex (or combination of sign complexes) in which something is signified in the identificative mode of signifying and in some other mode of signifying (designative, appraisive, prescriptive, or formative). Ascriptors are accordingly 
designative, appraisive, prescriptive, and formative. An ascriptor is undifferentiated if the same sign-vehicle is in the various modes of signifying; otherwise differentiated. When all the signs in an ascriptor are lansigns, the ascriptor is a lanascriptor.

B

Behavior $\ldots \ldots \ldots \ldots \ldots \ldots$ This term is presupposed by semiotic and not defined within it. Roughly speaking, behavior consists of the sequences of responses (actions of muscles and glands) by which an organism seeks goal-objects that satisfy its needs. Behavior is therefore "purposive" and is to be distinguished from response as such and from the even wider class of reactions. Behavior is individual or social, and when social may be co-operative, competitive, or symbiotic.

*Behavior-family $\ldots \ldots \ldots \ldots$ Any set of response-sequences that are initiated by similar stimulus-objects and that terminate in these objects as similar goal-objects for similar needs.

BeHAvioristics ............ The study of the behavior of organisms. 'Behavioristics' is a more general term than 'behaviorism,' the latter being a particular theory about the behavior of organisms.

BeLIEF $\ldots \ldots \ldots \ldots \ldots \ldots$ An interpreter is said to believe that an ascriptor is a $\mathrm{T}$-ascriptor or is reliable to the degree that he is disposed to act as if the ascriptor denotes or is reliable. Belief in general is not here defined.

C

Communicatee An organism that interprets a sign produced by a communicator.

Communication When restricted to signs, communication is the arousing of common significata by the production of signs. It is language communication when the signs produced are 
language signs. Not all communication is language communication.

Communicator $\ldots \ldots \ldots \ldots \ldots$ An organism that produces a sign that is a stimulus in social behavior to some organism (the communicatee).

*Comsign

A sign that has the same signification to the organism that produces it that it has to other organisms. Comsigns are a special class of interpersonal signs, since not all interpersonal signs are comsigns. Comsigns may be either comsignals or comsymbols.

Connector $\ldots \ldots \ldots \ldots \ldots \ldots$ A formator that establishes a particular connection between the interpretants of other signs in the given sign combinations in which it appears. There are intraascriptor and inter-ascriptor connectors.

CONTRADICTORY INTERPRETANTS ... Interpretants so related that if one issues in overt behavior, that fact alone prevents the other from issuing in overt behavior.

D

*Denotatum . ............. Anything that would permit the completion of the response-sequences to which an interpreter is disposed because of a sign.

*Denote

A sign that has a denotatum or denotata is said to denote its denotatum or denotata. All signs signify, but not all signs denote.

Descriptors .............. Identifiors that describe a spatial or temporal location.

*Designator

A sign that signifies characteristics or stimulus-properties of stimulus-objects. Such a significatum is called a discriminatum. Monadic, dyadic, and triadic designators are distinguished; also, objectdesignators and character-designators.

DETERMINOR

A formator that determines whether the interpretant of a general sign is to remain general or is to be restricted in its gener- 
ality in the given sign combination in which the formator appears.

Discriminatum ............. A significatum of a designator.

*Disposition to RESPOND ........ The state of an organism at a given time (RESPONSE-DISPOSITION) such that under certain additional conditions a given response takes place.

Dominant sign $\ldots \ldots \ldots \ldots$. The sign in an ascriptor whose interpretant is not subordinated to other interpretants; its mode of signifying determines the mode of signifying of the ascriptor.

$\mathrm{E}$

Expressive sIGN ............A sign is expressive if the fact of its production is itself a sign to its interpreter of something about the producer of the sign. Any sign may be expressive.

F

F-ASCRIPTOR ............... An ascriptor that does not denote. Ascriptors, not F-ascriptors, are $T$-ascriptors.

*Formative ascriptor A compound ascriptor such that the denotation of one or more of the component ascriptors (called the antecedent ascriptors) is, because of the signification of the ascriptor, a sufficient condition for the denotation or lack of denotation of the remaining component ascriptor or ascriptors (called the consequent ascriptors), and hence, for the denotation or lack of denotation of the compound ascriptor itself. Formative ascriptors are accordingly either analytic or contradictory.

*Formator $\ldots \ldots \ldots \ldots \ldots \ldots \ldots$ A sign signifying how something is signified in an ascriptor. Stated behaviorally, formators are signs that dispose their interpreters to modify in determinate ways the dispositions to response occasioned by other signs in the sign combinations in which the formator appears. The significatum of a formator is called a formatum. 
Determinors, connectors, and modors are kinds of formators.

Formatum . . . . . . . . . . . . . A significatum of a formator.

Formulization

A formative ascriptor considered as produced by some interpreter.

G

General sign .............. sign that is not singular. There are various degrees of generality depending upon the interrelationship of significata.

Goal-object ...............An object that partially or completely removes the state of an organism (the need) which motivates response-sequences.

I

ICONIC $\operatorname{sign} \ldots \ldots \ldots \ldots \ldots \ldots \ldots$ A sign is iconic to the extent to which it itself has the properties of its denotata; otherwise non-iconic.

*Identifior $\ldots \ldots \ldots \ldots \ldots \ldots$ A sign that signifies a location in space or time. Such a significatum is called a locatum. Indicators, descriptors, and namors are kinds of identifiors.

*INCITIVE USE OF SIGNS. ......... When signs are produced to call out more or less specific ways of responding to something. Signs incitively adequate are called persuasive.

INDICATORS $\ldots \ldots \ldots \ldots \ldots \ldots$ Identifiors which are non-language signals.

*INFORMative uSE of signs ..... When signs are produced to cause someone to act as if something had, has, or will have certain characteristics (discriminata, stimulus-properties). Signs informatively adequate are said to be convincing; they need not be true.

*Inter personal sign ........... A sign is interpersonal to the degree that it has the same signification to a number of interpreters; otherwise personal.

*INTERpretant ............. The disposition in an interpreter to respond, because of a sign, by responsesequences of some behavior-family. 
*INTERPRETER An organism for which something is a sign.

INTERPRETER-FAMILY $\ldots \ldots \ldots \ldots$ The interpreters for whom a sign is interpersonal.

K

KNOWLEDGE An interpreter is said to know that an ascriptor is a T-ascriptor or has reliability $n$ to the degree he has evidence that the ascriptor denotes or has reliability $n$. Knowledge in general is not here defined.

L

*Language See LANSIGN SYSTEM.

*Language sign See LANSIgN.

*Lansign A sign that is a member of a lansign-system. In this book 'language sign' is often used in place of 'lansign'; strictly speaking, only the latter term is defined.

*LaNSign-system A set of plurisituational signs with interpersonal significata common to members of an interpreter-family, the signs being producible by members of the interpreterfamily and combinable in some ways but not in others to form compound signs. Stated in terms of comsigns: a lansignsystem is a set of comsign-families the members of which are restricted in the ways in which they may be combined. In this book 'language' is often used in place of 'lansign-system'; strictly speaking, only the latter term is defined.

*Lexicative ascriptor Any ascriptor that is not a formative ascriptor.

*Lexicator Any sign that is not a formator; hence any identifior, designator, appraisor, or prescriptor.

Linguistics (GENERAL) ........A possible term for that part of semiotic which studies lansign-systems. Linguists are free to restrict the term 'linguistics' to 
some sub-set of such systems (such as those whose sign-vehicles are sounds produced by organisms).

Locatum

A significatum of an identifior.

LOCUS OF CONFIRMATION

The time and place at which evidence is found that an ascriptor is a T-ascriptor or an F-ascriptor, or reliable or unreliable. It is to be distinguished from the locus of signifying and the locus signified.

Locus of SIgNifying $\ldots \ldots \ldots$. The time and place at which a sign-process occurs. It is to be distinguished from the locus signified and the locus of confirmation.

Locus sIGNIFIED ............ The time and place signified in a signprocess. It is to be distinguished from the locus of signifying and the locus of confirmation.

Logic .................. A possible term for that part of semiotic composed of analytic formative ascriptors. Logical analysis would then consist of those sentences about signs which are analytic formative ascriptors in the semiotical metalanguage.

\section{M}

MEANS-OBJECT

An object that serves as a means toward the attainment of a goal-object.

*Mode of SIgNifying . ......... A differentiation of signs in terms of the most general kinds of significata. Five modes of signifying are distinguished (identificative, designative, appraisive, prescriptive, and formative), and signs signifying in these modes are called respectively identifiors, designators, appraisors, prescriptors, and formators.

Modor

A formator that establishes the mode of signifying of the sign combinations in which it appears. 
NAMOR ................... Identifiors that are language symbols, and hence, substitute signs synonymous with other identifiors.

NeEd $\ldots \ldots \ldots \ldots \ldots \ldots \ldots$. Here used as roughly synonymous with 'organic state motivating behavior,' and hence, distinguished from what is necessary for an organism to survive.

$\mathrm{O}$

Obligatum

A significatum of a prescriptor.

Pathic sign $\ldots \ldots \ldots \ldots \ldots$. A sign that is abnormally resistant to replacement by more adequate signs because of some partial satisfaction which the sign gives to its interpreter. Not all inadequate signs are pathic.

Personal sign ............... A sign is personal to the degree that it is not interpersonal.

Plurisituational sign ......... A sign that is not unisituational.

*Pragmatics $\ldots \ldots \ldots \ldots \ldots$. That branch of semiotic which studies the origin, the uses, and the effects of signs. It is distinguished from semantics and syntactics.

*Post-language symbols ....... Symbols producible by their interpreters and synonymous with language signs (lansigns). Such symbols may be personal or interpersonal, and may or may not themselves become elements in a language (lansign-system).

PRECISE SIGN Signs, not vague, are precise.

*Preparatory-stimulus ........A stimulus that influences a response to some other stimulus. A preparatory-stimulus necessarily causes at the time of stimulation a reaction in the organism for which it is a stimulus, but this reaction need not be a response (an action of a muscle or gland). 
Prescription A prescriptive ascriptor considered as produced by some interpreter.

*Prescriptor A sign that signifies the requiredness of certain response-sequences. A significatum of such a sign is called an obligatum. Hypothetical, categorical, and grounded prescriptors are distinguished.

\section{$\mathbf{R}$}

*ReLiable SIGN A sign is reliable to the degree that members of the sign-family to which it belongs denote; otherwise unreliable.

RESPONSE Any action of a muscle or gland. Hence, there are reactions of any organism which are not responses.

*RESPONSE-DISPOSITION See DISPOSITION TO RESPOND.

*RESPONSE-SEQUENCE Any sequence of consecutive responses whose first member is initiated by a stimulus-object and whose last member is a response to this stimulus-object as a goalobject (an object that partially or completely removes the state of the organism that motivates the sequence of responses).

\section{$S$}

*Semantrcs ................ That branch of semiotic which studies the signification of signs. It is distinguished from syntactics and pragmatics.

Semiosis A sign-process, that is, a process in which something is a sign to some organism. It is to be distinguished from semiotic as the study of semiosis. The terms 'semiosical' and 'semiotical' may be distinguished in a similar way.

*Semiotic The science of signs. Its main subdivisions are semantics, syntactics, and pragmatics. Each of these, and so semiotic as a whole, can be pure, descriptive, or applied. Pure semiotic elaborates a language to talk about signs, descriptive semiotic studies actual signs, and applied semiotic utilizes 
knowledge about signs for the accomplishment of various purposes.

Semiotician ............... A student of semiotic, the science of signs.

* SIGN $\ldots \ldots \ldots \ldots \ldots \ldots \ldots$ Roughly: something that directs behavior with respect to something that is not at the moment a stimulus. More accurately: If $A$ is a preparatory-stimulus that, in the absence of stimulus-objects initiating response-sequences of a certain behaviorfamily, causes in some organism a disposition to respond by response-sequences of this behavior-family, then $A$ is a sign. Anything that meets these conditions is a sign; it is left undecided whether there are signs that do not meet these conditions.

*Signal

A sign that is not a symbol, that is, not produced by its interpreter and not a substitute for some other sign with which it is synonymous.

Sign-BEhavior $\ldots \ldots \ldots \ldots \ldots$ Behavior in which signs occur.

*Sign-family $\ldots \ldots \ldots \ldots \ldots \ldots$ A set of similar sign-vehicles that for a given interpreter have the same signification.

*Significatum $\ldots \ldots \ldots \ldots \ldots$ The conditions such that whatever meets these conditions is a denotatum of a given sign. The formulation of what a sign signifies is called a formulated significatum. No attempt has been made to differentiate 'signification' and 'significatum.'

*SIGNIFY To signify is to act as a sign in a process of semiosis. 'To have signification' and 'to have a significatum' are synonymous with 'to signify.' A sign is said to signify (but not denote) its significatum, that is, the conditions under which it denotes. All signs signify; not all signs denote.

* Signifying, mode of ........... See mode of signifying.

* Sign-vehicle .............. A particular event or object, such as a sound or mark, that functions as a sign. 
SINGULAR SIGN

A sign whose signification permits only one denotatum; otherwise it is general.

Statement $\ldots \ldots \ldots \ldots \ldots \ldots \ldots$ A designative ascriptor considered as produced by some interpreter.

Strmulus Any physical energy that acts upon a receptor of a living organism. A stimulus causes a reaction in an organism, but not necessarily a response (a reaction of a muscle or gland).

Stimulus-OBJeCt ............ The source of a stimulus. The properties of the object that produce stimuli are called stimulus-properties.

*Srmbor

A sign that is produced by its interpreter and that acts as a substitute for some other sign with which it is synonymous; all signs not symbols are signals. Symbols may be pre-language, language, and post-language symbols.

*Synonymous signs $: . . \ldots \ldots$. Signs that belong to different sign-families and yet have the same signification.

SYNTHETIC ASCRIPTOR Any lexicative ascriptor.

*SYSTEMIC USE OF SIGNS When signs are produced to organize behavior that other signs tend to provoke. Signs systemically adequate are called correct.

*SYNTACTICS That branch of semiotic that studies the way in which signs of various classes are combined to form compound signs. It abstracts from the signification of the signs it studies and from their uses and effects; hence, it is distinguished from semantics and pragmatics.

$\mathrm{T}$

T-ASCRIPTOR An ascriptor that denotes. ' $T$ ' is used to suggest 'true' though the latter term is not here defined. Similarly, an F-ascriptor is one that does not denote. Ascriptors in any mode of signifying may be $T$-ascriptors or F-ascriptors. 
*TYPE OF DISCOURSE

A specialization of language for the accomplishment of specific purposes. The types of discourse are here classified on a mode-use basis, that is, in terms of the combination of some dominant mode of signifying with some dominant use. Thus, there are sixteen major types of discourse: designative-informative, designative-valuative, appraisive-incitive, formative-systemic, and so forth.

\section{$\mathrm{U}$}

UNAMBIGUOUS sIGN ...........A sign-vehicle is unambiguous when it has only one significatum, that is, belongs to only one sign-family; otherwise it is ambiguous.

UNistruational SIgN .......... A sign that signifies a given significatum in only one situation; hence, it is a signvehicle that belongs to no sign-family. Most signs are plurisituational.

UNRELIABLE SIGN .............. A sign that is not reliable.

*USE OF A SIGN .............. A sign is used with respect to some goal if it is produced by an interpreter as a means of attaining that goal; a sign that is used is thus a means-object. Four main uses of signs are distinguished: the informative, valuative, incitive, and systemic. The use of a sign is not to be confused with its mode of signifying.

\section{V}

VAGUe SIGN

A sign is vague to a given interpreter to the degree that its significatum does not permit the determination of whether something is or is not a denotatum; otherwise it is precise.

*Valuative use of signs When signs are used to cause preferential behavior to something. Signs valuatively adequate are said to be effective. A significatum of an appraisor. 
INDEX 


\section{INDEX}

AMES of authors whose works appear in the bibliography have
not been included except as they occur in the text or the notes. The terms that are starred appear in the glossary.

Abelard, 286

* Adequacy, of signs, 122

Allport, F. H., 252, 253

Alternativity, property of, 157-158

* Ambiguous sign, 21

Analysis, logical, 181

* Analytic interpretants, 165

Andrade, Manuel J., 74, 143, 189, 221, 223, 280

Antedating response, of Hull, 307

Anticipation of needs, of Mowrer, 275

Anxiety, and socially pathic signs, 211

Appraisive ascriptors, 79-83

Appraisive-incitive discourse, 138-139

Appraisive-informative discourse, 134

Appraisive-systemic discourse, 140

Appraisive-valuative discourse, 136

*Appraisors, 66, 67, 79-83, 258

consummators, 83

negative, 82

positive, 82

utilitators, 83

Appropriate behavior, ambiguity of term, 16

Appropriate response, 294

Aquinas, Thomas, 141

Aristotle, 107, 141, 171, 176, 237, 271, 281, 286,297

Art, 192-196, 213

$\therefore$ Ascriptors:

appraisive, 74, 79

compound, 75

denotatum of, 106

designative, 74,76

differentiated, 73

F-ascriptors, as false, 107, 167

formative, $74,166-168,269$
Ascriptors (Cont.):

identificative, 75,76

lanascriptor, 73

lexicative, 153-158

prescriptive, 74, 83-86

synthetic, 163

T-ascriptor, as true, 106-108

undifferentiated, 73

Augustine, 286

Ayer, A. J., 265

\section{B}

Bacon, Francis, 287

Bacon, Roger, 286

Balkan, E. R., 277

* Behavior, 250 appropriate, ambiguity of term, 16 individual, 32 preferential, 137, 258 social, 32, 139, 252

* Behavior-family, 10, 251

Behavioral:

basis of formative ascriptors, 164-166

criteria for differentiating the modes, 6467

formulations of sign-processes, 291-294

semiotic, alternatives to, 28-31

Behavioral-mentalistic controversy, 299-301

Behaviorism, 28

* Behavioristics, 2

Being, 281

* Belief, 298, 299

and knowledge, 108-111

denotation, and adequacy, 121-122

Benjamin, A. C., 262

Bentham, Jeremy, 287

Bentley, Arthur F., 251 
Berkeley, George, 287

Bevan, E., 266

Bierens de Haan, J. A., 254

Black, M., 251

Bloomfield, Lconard, 189, 192, 221, 252, $265,279,280$

Boethius, 286

Brentano, Franz, 297

Brewster, John M., 254

Brill, A. A., 276

Britton, Karl, 257, 265, 268

Brunswik, Egon, 250, 251, 252

Buddha, 134

Bühler, Karl, 221, 257, 268

\section{C}

Carnap, Rudolf, 110, 182, 218, 221, 227, $251,257,262,265,268,270,271$, $272,279,280,281,287$

Cason, H., 292

Cassirer, Ernst, 135, 189, 221, 257, 264, 265, $271,274,281$

Censorship, motion pictures, 278

Chapple, E. D., 189, 206

Christianity, 147

Clayton, Alfred S., 254

* Communicatee, 32, 118

* Communication, 38, 54, 118-121, 212-216, $263,278,283$

adequacy of, 119

basic improvement in, 120-121

content of, 118

means of, 118

self-communication, 213

social, 213

* Communicator, 32, 118

Components of signifying:

appraisive, 62

designative, 62

prescriptive, 62

* Comsign, 33, 39, 253

Concept, 49, 52

Conditioning, 309

Confirmation, degree of, 110, 262

Conflict, 212-216

* Connectors:

inter-ascriptor, 160

intra-ascriptor, 160

Consciousness, 301

Constitution, 131

Context theories of signs, 16

Contradictory implicate, 22

* Contradictory interpretants, 165-166

Control of non-designative ascriptors, 114118
Convincingness: informative adequacy, 9799

Coon, C. S., 189, 206

Co-operation, 212-216

Co-operative social behavior, 212-216

Copilowish, I. M., 251

Correctness: systemic adequacy, 104-106

Cosmological discourse, 132-134

Courtrai, Siger de, 285, 286

Crawford, M. P., 255

Critical discourse, 140-142

Criticism, 140

adequacy of, 142

esthetic, 231

theory of, 266

Culture, 204, 207

Cunningham, G. Watts, 296

\section{D}

Dalkey, Norman, 262

Declaration of Independence, 145

Degree of confirmation, 110,262

DeLacy, E. A., 285

DeLacy, P. H., 285

DeLaguna, Grace, 252, 260

Delayed response, of Hunter, 293

Democratic control of agencies of communication, 242-243

Democratic society, 244

Democracy, 243

Denotation, belief, and adequacy, 121-122

* Denotatum, 17

* Denote, 17

* Descriptors, 76

Designation, in informative use of signs, 98

Designative ascriptors, 76-79

Designative-incitive discourse, $130-132$

Designative-informative discourse, $126-128$

Designative-systemic discourse, 132-134

Designative-valuative discourse, $128-130$

* Designators, 66-69, 76-79

character-designators, 78

dyadic, 78

monadic, 78

object-designators, 78

triadic, 78

Designatum, 258

* Determinor, 159

Dewey, John, 205, 251, 254, 257, 258, 262, $263,266,272,274,282,283$

Discourse:

classification of types, $123-150$

cosmological, 132-134

critical, $140-142$

fictive, $128-130$ 
Discourse (Cont.): formative, 153-186

four main modes, 123-126

four main uses, 123-126

grammatical, 173-175

legal, 130-132

logico-mathematical, 168-171

metaphysical, 175-178

mode-use classification, 125

moral, 138-140

mythical, 134-135

poetic, 136-138

political, 145-146

propagandistic, 148-150

religious, 146-148

rhetorical, 171-173

scientific, 126-128

study of types of, 150-152

technological, 143-144

* Discriminatum, 67, 77

* Disposition to respond, 9, 251

Doctrines, of the equality of men, 145

Dollard, John, 253

* Dominant sign, 74

Ducasse, C. J., 257, 262, 297, 300

\section{E}

Education, 246, 283

Effectiveness:

as differing from convincingness, 101

as valuative adequacy, 99-101

Emerson, Alfred E., 254, 255

Emotion, 61-62, 67-69

Emotive, 60, 61, 194, 264

Empirical philosophy, 282

Empiricism, 282

Empson, William, 265

Epicureans, 285

Epistemology, 262

Esthetic criticism, 231

Esthetic signs, 195, 274

Ethics, 141-142

Evaluation, 101, 140

Events, private, 227, 230

Evidence:

direct, 112-113

indirect, 112-113

Excitors and inhibitors, 259

Expectations, 121, 128, 303

Expression, emotion, and usage, 67-69

* Expressive:

aspect of a sign, 259

signs, 69, 194

usage of term 'expressive,' 257
* F-ascriptor, 107, 167

False, 107

Feigl, H., 94, 257, 261

Fiction, 128-130

Fictive discourse, 128-130

Formal sciences, 225

Formal signs, 86

Formal truth, 167

* Formative ascriptors, 86-88, 166-168 analytic, 163

behavioral basis of, $164-166$

contradictory, 163

synthetic, 163

various purposes of, $169-170$

Formative discourse, 153-186

formative-incitive discourse, 173-175

formative-informative discourse, 169-171

formative-systemic discourse, 176-178

formative-valuative discourse, 171-173

* Formators, 86-88, 153-186, 268

kinds of, 159-162

interpretant distinctive of, 157

problem of, 153-156

* Formatum, 88, 158, 259

* Formulization, 88, 166

Fractional antedating response, of Hull, 307

Frank, L. K., 283

Freedom, 197, 275

Frege, B., 287

French, T. M., 277

Frenkel-Brunswik, Else, 250

Freud, Sigmund, 189, 276

Fromm, Erich, 202, 277

Function, 261

Functions of language, of Ogden and Richards, 69-72

\section{G}

Gantt, W. Horsley, 253, 275

Gardiner, Alan H., 189, 221, 279

Gätschenberger, Richard, 251

Geisteswissenschaften, 230, 231, 281

General linguistics, 38

General sign, 22

Genesis of language, 256

Gentry, George V., 258

Gesture, 43

* Goal-object, 83

Goldstein, Kurt, 189, 277

Gomperz, H., 293

Good:

food signified as, 115

the term, 81,82

Gorgias, 172 
Gospels, 147

Grammar, 34, 174, 182-186, 222, 280

Grammatic, 183

Grammatical discoursc, 173-175

$\mathrm{H}$

Habit, 310

Hahn, Hans, 271, 280

Hahn, Lewis E., 252

Hayakawa, S. I., 283

Head, Henry, 189, 277

Heider, F., 274

Hempel, C. G., 251, 280

Hilgard, E. R., 259, 288

Hispanus, Petrus, 286

Hobbes, 287

Hofstadter, A., 251

Hollingworth, H. L., 291, 301

Hudgins, C. V., 275

Hull, Clark L., 8, 56-59, 189, 250, 252, $255,259,275,300,305-308,309$

Humanistics, 281

Humanitics and scientific humanistics, 230233

Hume, 231, 287

Hunter, W. H., 24, 251, 252, 292, 301

Husserl, Edmund, 251, 297

Hypnosis, 275

\section{I}

Ichheiser, Gustav, 263

Icon, 192, 276

* Iconic sign, 190-192, 201

Idea, 30,49

Idealism, 177

*Identifior, 66, 67, 76-79

Implicate relation of signs, 22,165

Import of semiotic:

for individual, 239-241

for social organization, 242-244

Import of signs, individual and social, 187216

Incitive adequacy: persuasiveness, 102-104

Incitive use of signs, 102-104

*Indicators, 76

Inform, 99

Informative adequacy: convincingness, 9799

* Informative use of signs, 97-99

Inhibitors, 259

* Interpersonal signs, 21

* Interpretant, 17, 30, 157, 275, 303

analytic, 165

and significatum, 18
Interpretant (Cont.):

contradictory, 165

of Peirce, 289

* Interpreter, 17

Interrelation of modes of signifying, $88-91$

Isogenous signs, 280

\section{J}

Jakobson, Roman, 280

James, Henry, 27

James, William, 281, 282

Janis, Irving L., 259

Jeliffe, S. E., 277

Jones, Ernest, 276

K

Kant, 141, 171, 282

Kantor, J. R., 252

Kaplan, Abraham, 142, 213, 257, 266, 279

Kasanin, J. H., 277

Kelly, W. H., 277

Kepes, G., 274

Kluckhohn, C., 277

* Knowledge: and belief, 108-111

formative ascriptors, truth, and, 166-168 limits of signification and, 111-114

Korzybski, Alfred, 251, 283

Krechel, J., 277

Kris, Ernst, 277

Kroeber, A. L., 254

Krout, M. H., 277

L

Langer, Susanne, 50, 189, 194, 251, 257

* Language, 174, 220

and behavioristics, $55-59$

and communication, 38

and social behavior, $32-59$

and social heredity, 254

as sign phenomenon, 32-35

definition of, $35-36$

genesis of, 256

of philosophy, 233-238

question of arts as language, 192-196

question whether language is social, 37 utterance, 37

* Language sign, interpersonality of, 39-42

* Lansign, 36

* Lansign-system, 36

Lashley, Karl, 249, 275

Lasswell, Harold D., 151, 189

Lee, Alfred, 266

Lee, Elizabeth, 266

Legal discourse, 130-132 
Leibniz, 257, 285, 286

Lepley, Ray, 263

Lewis, C. I., 251, 273, 281

* Lexicative ascriptors, 153-158

* Lexicators, 153-158

Limits of signification and knowledge, 111114

Linguistic science, 222

- Linguistics, 279

general, 38

program for, $220-223$

relation to semiotic, 220

Link, George K., 198, 275

Literature, 266

* Locatum, 67

Locke, John, 249, 281, 282

* Locus of confirmation, 111

* Locus of signifying, 111

- Locus signified, 111

Logic, 172, 181, 270-272

Logic and mathematics, 178-182

Logical analysis, 181

Logical signs, 86, 268

Logicians, symbolic, 269, 272

Logico-mathematical discourse, 168-171

Lying, 261-262

\section{$\mathbf{M}$}

Mace, C. A., 94, 257, 261

Magic, 143-144

Malinowski, Bronislaw, 135, 189, 205, 265, 277

Mannheim, K., 278

Markey, John F., 253, 255

Marquis, D. G., 259, 288

Masserman, Jules H., 189, 199, 202, 251, 255,277

Materialism, 177

Mathematics, 271

Mathematics and logic, 178-182

May, Mark, 208

Mead, George H., 39-49, 56, 139, 166, 189, $197,207,251,252,254,274,275$, $280,281,300$

concept of significant symbol, 42-46

taking the role of the other, 45

Mead, Margaret, 277-278

Meaning, 19

* Mcans-object, 82-83, 305

Mediated taking account of, of Morris, 251

Mental, 228

Mentalism, 28

Mentalistic-behavioral controversy, 49-52, 299-301
Metalanguage, 179

Metaphorical signs, 136, 276

Metaphysical discourse, 175-178

Metaphysics, 281

Metaphysics, rhetoric, and grammatic, 182184

Metasigns, 179

Meyer, Adolf, 189, 277

Mill, John Stuart, 141

Miller, Neal E., 253

Mind, 49, 301

* Modes of signifying, 60-91

behavioral criteria for differentiating, 64 67

differences in, 63

independence of, 89

interdependence of, 89

interrelation of, 88-91

origin of, 62-64

origin of differences in, 63

* Modor, 161

Moral discourse, 138-140

Morris, Charles, 251, 257, 266, 280, 283

Motion picture censorship, 278

Mowrer, O. H., 8; 249, 275

Music, 193, 194

Mythical discourse, 134-135

\section{$\mathrm{N}$}

* Namors, 76-77

Natural sciences, 225

* Needs, 8-9, 81, 83, 250

Neurath, Otto, 2, 217, 283

Newton, 225

Nissen, Henry W., 255, 310

Non-vocal signs, 190-192

Numerical expressions, 269

Object:

language, 179

of Peirce, 289

perception of, 274

Object signs, 179

* Obligatum, 67

Ogden, C. K., 257

Ogden, C. K., and Richards, I. A., 251, 261, $287,295,296$

classification of uses of signs, 93

emotive terms, $60,61,70-72$

modes of signifying, 69-72

referential terms, $60,61,70-72$

Oppenheim, Félix E., 265

Ought, 139 
P

Painting, 192, 193

Palmer, H. E., 264

Parentheses, 87

Park, Robert E., 212, 278

Parsons, Howard, 264

Parts of speech, 222

* Pathic sign, 200, 210, 241

Pathic:

event, 198

functioning of signs, 277

sign, 200, 241

socially pathic signs, 210

Pathology of signs, 198-201

Pavlov, I. P., 251, 253, 291

Peano, G., 287

Peirce, Charles S., 27, 194, 221, 249, 251, $271,272,274,281,287,288-291$

Pepper, Stephen, 265

Perception, 34, 191, 252

of objects, 274

* Perşonal signs, 21

Personality disturbances and signs, 201-204

Persuasive definition, of C. L. Stevenson, 149

Persuasiveness: incitive adequacy, 102-104

Phenomenology, 30

Philosophic synthesis, 235

Philosophy, 235, 281, 282, 301

empirical, 282

intimate relation to semiotic, 237

language of, 233-238

not a part of semiotic, 237

Phoneme, 222

Piaget, Jean, 277

Plato, 286

* Plurisituational sign, 21

Poetic discourse, 136-138

Poetry, 100, 136, 265

Pointing, 258

Political discourse, 145-146

Pollock, T. C., 93, 257, 261, 266

* Post-language symbols, 46-49 personal, 17, 196-198

effects of, 196-198

* Pragmatics, 279

Pragmatics, semantics, and syntactics, 217. 220

Prantl, Carl von, 285

* Precise sign, 21

Predictions, 128

Preferable, 80

Preferential behavior, 79.81, 137, 258

Preferential status, 81

* Preparatory-stimulus, 8
* Prescription, 86

Prescriptive ascriptors, $83-86$

* Prescriptors, 66-69

and prescriptive ascriptors, $83-86$

categorical, 85

grounded, 85

hypothetical, 85

requiredness of certain actions, 84

Private events, $227-230$

Probability, 110, 262

Problems and program of science of signs, 246-248

Propagandistic discourse, $148-150$

Property, the term, 81

Proposition, 262

Psychiatry, 277

Psychoanalysis, 276, 277

Psychology, 301

place of, within science, $227-230$

Pure-stimulus acts, of Hull, 305-308

\section{Q}

Quine, W. V., 269

$\mathbf{R}$

Range, 272

Ransom, J. C., 265, 274

Raup, B., 283

Reciprocal social behavior:

competitive, 32

co-operative, 32

symbiotic, 32

Referential terms, of Ogden and Richards, $60,61,69.72$

Reichenbach, H., 94, 221, 257, 261, 262, 269,279

* Reliable signs, 23, 106, 121

Religious discourse, $146-148$

Religious signs, 264

Representamen, of Peirce, 289

- Response, 8

antedating, of Hull, 307

appropriate, 294

delaycd, of Hunter, 293

fractional antedating, of $\mathrm{Hull}, 307$

* Response-disposition, 9

* Response-sequence, 9-11, 13

Rhetoric, 183

Rhetoric, grammatic, and metaphysics, 182184

Rhetorical discourse, 171-175

Richards, I. A., 231, 257, 263, 265, 296

Riesen, A. H., 255

Riezler, K., 283

Role-taking, of Mead, 45, 254 
Rousseau, 145

Russell, Bertrand, 225, 257, 279, 282, 287

\section{5}

Saiving, Valeric, 266-267

Sanford, Fillmore H., 276-277

Santayana, George, 282

Sapir, Edward, 189, 221, 252, 257, 274, 277,279

Sceptics, 285

Schlick, Moritz, 281

Science, 180

and the humanities, 246

of signs, 4-5

of signs, problems and program, 246-248

place of psychology within, 227-230

Scientia sermocinalis, 249,286

Scientific discourse, 126-128

Scientific humanistics, the humanities and, 230-233

Self-communication, 213

Self-expressive sign, 213

Semantic concept of truth, 262

- Semantics, 2, 249

Semantics, pragmatics, and syntactics, 217 220

- Semiosis, of Peirce, 287

Semiotic, 2, 180, 249

and the school, 244-246

and the social-psychological disciplines, 188-189

applied, 220

as unification of science, $223-227$

basic terms of, $17-20$

history of, 285-287

import of, for individual, 239-241

import of, for social organization, 242244

not a theory of value, 80

philosophy intimately related to, 237

philosophy not a part of, 237

pure and descriptive, of Carnap, 219

relation of linguistics to, 220

scope and import of, 217, 248

the term, 285

- Semiotician, 4

Senior, James K., 251

Sentence, 73, 222, 262

Sheffer, H. M., 257, 287

Sheldon, W. H., 209, 276

Shelley, 136

- Sign-behavior: precision in identification of, 8-11 preliminary isolation of, $5-7$ uniqueness of, 308-310
* Sign-family, 20, 251

Sign-gestalt, of Tolman, 302-305

Sign-processes, 15-17

behavioral formulations of, 291-294

healthy, 199

* Sign-vehicle, 20

Signal and symbol, 23-27, 49

Signal process, 25

Significant symbol, of Mead, 42-46, 253

Significata, terms for kinds of, 66-67

Signification, limits of knowledge and, 111114

Significatum, 17, 20

* Signify, 17

* Signifying, modes of, 60-91

Signifying, of past occurrences, 112

* Signs:

about signs (metasigns), 179

adequacy, truth, and reliability of, 92-122

and personality disturbances, 201-204

and societies, 204-207

as analytic implicates, 22, 165

as comsign, 33

as contradictory implicates, 22, 165

as used with respect to purpose, 92

classification of, 61-62

conditions sufficient for, 10

context theories of, 16

dominant, 74

esthetic, 195, 274

evidence of conditions sufficient for, 1315

expressive, 68

formal, 86

general, 22

iconic, 23, 191, 201

in animals and men, 52-55

in early behavior theory, 6

individual and social import of, 187216

interpersonal, 21

isogenous, 280

lexicative, 153,156

logical, 86, 268

metaphorical, 136, 276

non-iconic, 23

non-vocal, 190-192

pathic, 200, 241

pathic functioning of, 277

pathology of, 198-201

personal, 21

personal post-language, 47, 196-198

plurisituational, 21

precise, 21

purposes of, 68 
Signs (Cont.):

reliable, $23,106,121$

religious, 264

science of, 4-5, 246-248

self-expressive, 213

singular, 22

social pathology of, $210-212$

socially pathic, 210

syncategorematic, $86,259-260$

synonymous, 22

unisituational, 21

universal, 22

unreliable, 23

uses of, 93-106

vague, 21

visual, 190

Simplicius, 172

Singer, Milton B., 278

* Singular sign, 22

Skinner, B. F., 250, 281, 309

Social behavior: and moral discourse, 139

competitive, 32

co-operative, 32,215

language and, 32-59

non-reciprocal, 32

reciprocal, 32

symbiotic, 32

Social control, $207-210$

democratic, 242

of agencies of communication, 243

totalitarian, 242

Social heredity and language, 254

Social pathology of signs, $210-212$

Social sciences, 281

Societies and signs, 204-207

Society, 242

democratic, 209, 244, 246

healthy, 210

totalitarian, 209, 244

Socrates, 140

Spinoza, 141

* Statement, 79

Stevenson, Charles L., 93, 149, 250, 257, 261,265

- Stimulus, 8

equivalence, of Hull, 57

generalization, of Hull, 57

patterning, of Hull, 56

preparatory, 8

- Stimulus-object, 8-11

Stoics, 249, 281, 285

Stravinsky, Igor, 193

Styles of writing, 264

Sullivan, Harry Stack, 189, 202, 277
* Symbol, 306

and signal, 23-27

in Freudian sense, 276

literary, 26

post-language, 46-49

religious, 26

significant, of Mead, 42-46, 253

Symbol-process, 25

Symbolic logicians, 269, 272

Syncategorematic signs, $86,259-260$

* Synonymous signs, 22

* Syntactics, 279

Syntactics, pragmatics, and semantics, 217 220

Synthesis, philosophic, 235

* Synthetic ascriptor, 163

* Systemic use of signs, 104-106, 234, 267 adequacy: correctness, 104-106

\section{$\mathrm{T}$}

* T-ascriptors, 167

and truth, 106-108

appraisive, 107

designative, 107

formative, 107

prescriptive, 107

Taking the role of the other, of Mead, 45

Tarski, Alfred, 250, 262, 287

Technological discourse, 143-144

Terminology, extension of, 20-23

Theology, 141, 147

Theory:

of criticism, 266

of types, 279

of value, 263

Thomas of Erfurt, 286

Thought, 196, 301

Tolman, Edward C., 56, 189, 252, 255, 300,309

concept of sign-gestalt of, 302-305

Totalitarian:

control of agencies of communication, 243 society, 242, 244

Truth, 97, 163, 166-168

and reliability, 109

semantic concept of, 262

* Types of discourse, 123-152

\section{U}

- Unambiguous sign, 21

Understanding, 253

Unification of science, 223-227

* Unisituational sign, 21 
Unity of science movement, 225

* Unreliable signs, 23

Urban, Wilbur, 189, 257, 264, 265, 281, 297

Usage, $67-69$

* Use of a sign:

classification of uses, 93

incitive, 95, 102-104

informative, $95,97.99$

primary use of appraisors, 96

primary use of designators, 96

primary use of formators, 96

primary use of prescriptors, 96

systemic, 95, 104-106, 234

the term 'use,' 96

valuative, $95,99-101$

Utopias, 130

\section{V}

* Vague sign, 21

Valuation, 259

Valuative adequacy: effectiveness, 99-101

* Valuative use of signs, 96, 99-101

* Valuatum, 67, 79, 81

Value theory, 263

Variables, 269

Verlaine, L., 255
Vieta, F, 286

Visual signs, 190

w

Wallerand, G., 285

Warner, W. L., 189

Watson, J. B., 47, 252, 275

Weiss, A. P., 252

Wertheimer, Max, 191

Whetnall, E. M., 251

Whitehead, A. N., 225, 281, 287

Whitman, 136, 141

Wick, W. A., 271

Wieman, Henry N., 266

Williams, K. A., 250

William of Ockham, 286

Willoughby, R. R., 275

Wittgenstein, Ludwig, 270, 271

Word, 222

Writing, styles of, 264

\section{$\mathrm{Y}$}

Yerkes, Robert M., 24, 251, 254, 255

Z

Zener, Karl, 249

Zeno, 172 